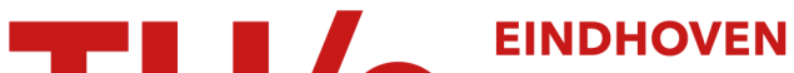 \\ UNIVERSITY OF \\ TECHNOLOGY
}

\section{Sound concentration caused by curved surfaces}

Citation for published version (APA):

Vercammen, M. L. S. (2012). Sound concentration caused by curved surfaces. [Phd Thesis 1 (Research TU/e / Graduation TU/e), Built Environment]. Technische Universiteit Eindhoven. https://doi.org/10.6100/IR732483

DOI:

10.6100/IR732483

Document status and date:

Published: 01/01/2012

\section{Document Version:}

Publisher's PDF, also known as Version of Record (includes final page, issue and volume numbers)

\section{Please check the document version of this publication:}

- A submitted manuscript is the version of the article upon submission and before peer-review. There can be important differences between the submitted version and the official published version of record. People interested in the research are advised to contact the author for the final version of the publication, or visit the $\mathrm{DOI}$ to the publisher's website.

- The final author version and the galley proof are versions of the publication after peer review.

- The final published version features the final layout of the paper including the volume, issue and page numbers.

Link to publication

\section{General rights}

Copyright and moral rights for the publications made accessible in the public portal are retained by the authors and/or other copyright owners and it is a condition of accessing publications that users recognise and abide by the legal requirements associated with these rights.

- Users may download and print one copy of any publication from the public portal for the purpose of private study or research.

- You may not further distribute the material or use it for any profit-making activity or commercial gain

- You may freely distribute the URL identifying the publication in the public portal.

If the publication is distributed under the terms of Article $25 \mathrm{fa}$ of the Dutch Copyright Act, indicated by the "Taverne" license above, please follow below link for the End User Agreement:

www.tue.nl/taverne

Take down policy

If you believe that this document breaches copyright please contact us at:

openaccess@tue.nl

providing details and we will investigate your claim. 


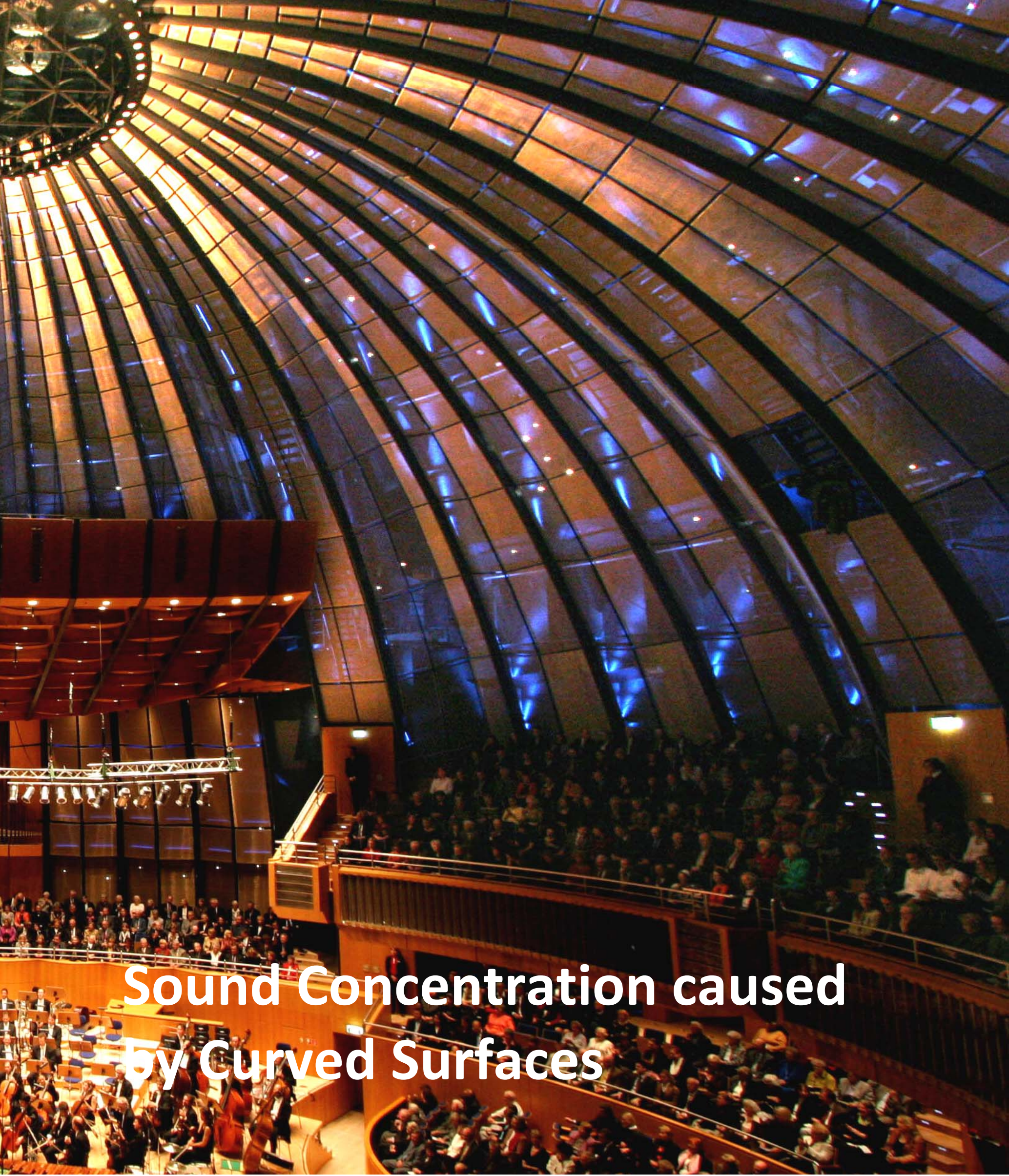




\section{SOUND CONCENTRATION CAUSED BY CURVED SURFACES}

Martijn Vercammen 
De promotiecommissie is als volgt samengesteld:

\begin{tabular}{|c|c|c|}
\hline $\begin{array}{l}\text { Voorzitter } \\
\text { 1e promoter } \\
\text { 2e promoter } \\
\text { copromotor } \\
\text { leden: }\end{array}$ & $\begin{array}{l}\text { prof.dr.ir. B. de Vries } \\
\text { prof.ir. E. Gerretsen } \\
\text { prof.ir. L.C.J. van Luxemburg } \\
\text { dr.ir. H.J. Martin } \\
\text { prof.dr.rer.nat. M. Vorländer } \\
\text { prof.dr.ir. G.L. Vermeir } \\
\text { prof.dr. A.G. Kohlrausch } \\
\text { prof.dr.ir. D. de Vries }\end{array}$ & $\begin{array}{l}\text { Technische Universiteit Eindhoven } \\
\text { Technische Universiteit Eindhoven } \\
\text { Technische Universiteit Eindhoven } \\
\text { Technische Universiteit Eindhoven } \\
\text { RWTH Aachen } \\
\text { Katholieke Universiteit Leuven } \\
\text { Technische Universiteit Eindhoven } \\
\text { RWTH Aachen (voorheen TU Delft) }\end{array}$ \\
\hline
\end{tabular}

Sound concentration caused by curved surfaces

Vercammen, Martijn

PhD thesis, Eindhoven University of Technology

Subject headings: Acoustics, Architectural acoustics, Sound-waves

ISBN: 978-90-6814-646-2

NUR-code: 950

URL: http://repository.tue.nl/

Copyright $@ 2012$ by M.L.S. Vercammen, Nijmegen, The Netherlands.

Printed by Eindhoven University press, Eindhoven, The Netherlands.

Cover: Tonhalle Düsseldorf, after renovation (photograph: Bernd Nanninga).

Published as issue 163 in the Bouwstenen series of the Faculty of Architecture, Building and Planning of the Eindhoven University of Technology.

This research project was supported by Peutz BV. This support is gratefully acknowledged.

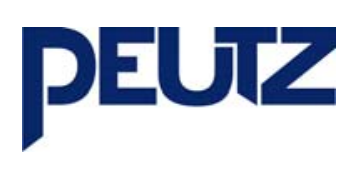

\section{TU/e}




\title{
SOUND CONCENTRATION CAUSED BY CURVED SURFACES
}

\author{
PROEFSCHRIFT
}

ter verkrijging van de graad van doctor aan de Technische Universiteit Eindhoven, op gezag van de rector magnificus, prof.dr.ir. C.J. van Duijn, voor een commissie aangewezen door het College voor

Promoties in het openbaar te verdedigen op dinsdag 3 april 2012 om 16.00 uur

door

Martinus Leonardus Servatius Vercammen

geboren te Waalwijk 
Dit proefschrift is goedgekeurd door de promotoren:

prof.ir. E. Gerretsen

en

prof.ir. L.C.J. van Luxemburg ${ }^{\dagger}$

Copromotor:

dr.ir. H.J. Martin 


\section{Preface}

In 2004 the author was involved, as the acoustical consultant of Peutz, in the renovation of the Tonhalle concert hall in Düsseldorf, Germany. This concert hall had basically a spherical (cupola) shape with a minimal diffusing structure and suffered a severe echo (see par.14.3). The first solution concept proposed included a high degree of diffusion on this cupola. However, scale model research showed that this would not sufficiently remove the echo. This conclusion was at that time rather surprising and disappointing. In the search for an explanation and better alternatives it became clear that there is hardly any information in room acoustics literature on the topic of sound concentration. After many tests, including scale model diffusing tests, the solution was found to be redirecting the reflected sound. This solution was realized in the real hall, which opened after renovation in 2005 with a concert work for percussion. The echo was not heard and the problem was solved.

Despite the fact that the problem was solved for this particular case, the lack of information concerning sound focusing was still there. So in 2006 the work on this dissertation began and it became clear why the Tonhalle echo problem could not be solved with diffusion.

Hopefully the results described in this work will enhance understanding of focusing in room acoustics and will help to solve this kinds of problems, preferably in the design stage.

\section{Reading instruction and notation conventions:}

Decimal numbers are indicated with a comma.

References to formulas are indicated by the formula number in between brackets (3.13)

The function $\lg ()$ refers to the logarithm with base 10.

The text in grey boxes contains notes, (long) derivations or notes on accuracy and application boundaries. These may be skipped by the reader.

For practical use of the results of this work: sections II,III and IV may be skipped, the most important formulas are summarised in chapter 12 . 


\section{Samenvatting}

\section{GELUIDCONCENTRATIE VEROORZAAKT DOOR GEKROMDE OPPERVLAKKEN}

In de zaalakoestiek is focussering, veroorzaakt door geluidreflecties tegen concaaf gekromde vlakken, een bekend probleem. Ofschoon gekromde vlakken in de hele geschiedenis van het bouwen gevonden kunnen worden, komen ze in de moderne architectuur steeds meer voor, dankzij hedendaagse ontwerp-, materiaal- en fabricagetechnieken. Focussering kan plaatselijk leiden tot onder meer zeer hoge geluidniveaus, kleuring of een echo. Alhoewel het fenomeen bekend is, is dit niet het geval voor de mate van versterking in het focusseringspunt en het geluidveld rondom het focusseringspunt.

Op grond van onderzoek naar geometrische methoden kan geconcludeerd worden dat deze niet gebruikt kunnen worden voor een kwantificering van de geluiddruk in het focusseringspunt. Dit is slechts mogelijk met een golfveldbenadering.

In voorliggende studie worden een aantal uitdrukkingen afgeleid voor de sterkte van geluidreflecties van concaaf gekromde vlakken, op basis van de Kirchhoff Integraal. De berekeningsmethodiek is geverifieerd aan de hand van een laboratoriumexperiment. Voor het geluidniveau in en rond het focusseringspunt wordt een benaderingsmethode gepresenteerd. Dit maakt het mogelijk de echo te beoordelen en het ontwerp te verbeteren of te herzien.

In het focusseringspunt is de hoogte van de geluiddruk met name afhankelijk van de golflengte. De afmetingen van het focusseringsgebied hangen eveneens samen met de golflengte. Bij kleine golflengtes is de geluiddruk hoog maar het focusseringsgebied klein, bij grote golflengtes is de geluiddruk lager, maar het focusseringsgebied groter.

De focussering ten gevolge van in twee richtingen gekromde vlakken (bol, ellipsoïde) is beduidend sterker dan die van in één richting (cilinder). In het algemeen zal de bereikbare reductie van de focussering door toepassing van absorptie of diffusie bij in twee richtingen gekromde vlakken onvoldoende zijn om de geluidconcentratie geheel weg te nemen. Bij cilindrische vlakken zal dit mogelijk wel voldoende kunnen zijn.

In het geval geluidabsorptie of diffusie onvoldoende soelaas biedt dient gedacht te worden aan het in een andere richting reflecteren met schuin geplaatste klankkaatsers of meer ingrijpend, aan herziening van het ontwerp.

Bij voorkeur dienen mogelijke focusseringseffecten ten gevolge van gekromde vlakken vanaf het begin van het ontwerp beschouwd te worden. 


\section{Summary}

\section{SOUND CONCENTRATION CAUSED BY CURVED SURFACES}

In room acoustics the focusing effect of reflections from concave surfaces is a wellknown problem. Although curved surfaces are found throughout the history of architecture, the occurrence of concave surfaces has tended to increase in modern architecture, due to new techniques in design, materials and manufacturing. Among other things, focusing can cause high sound pressure levels, sound coloration or an echo. Although the problem is known, the amount of amplification that occurs in the focusing point and the sound field around the focusing point are not.

It has been found that geometrical methods cannot be used to calculate the sound pressure in the focusing point. This pressure can only be calculated using wavebased methods. This work provides mathematical formulations for sound reflections from concave surfaces, based on the Kirchhoff Integral. This method is verified with an experiment.

An engineering method is given to approximate the sound field in and around the focusing point. This enables designers to evaluate and thereby improve or redesign the geometry.

In the focusing point the pressure depends on the wavelength. The width of the peak pressure is also related to the wavelength. For small wavelengths the amplification is high but the focusing area is small, while for lower frequencies the amplification is lower, but the focusing area is larger. The focusing caused by surfaces that are curved in two directions (sphere, ellipsoid) is much stronger than that caused by surfaces that are curved in only one direction (cylinders). Generally, the possible reduction of the focusing effect that can be achieved by using absorbers or diffusers is not enough to eliminate the focusing effect of double curved surfaces. However, these methods might be sufficient to reduce the focusing caused by single curved surfaces such as cylindrical shapes. If absorption or diffusion is not sufficient, designers can consider either redirecting the reflections or more drastically revising the geometry. It would be preferable to consider the focusing caused by concave surfaces from the early design stages. 


\section{contents}

Preface

Samenvatting

Summary

$\mathrm{V}$

vi

vii

I. INTRODUCTION INTO SOUND CONCENTRATION

$\begin{array}{ll}\text { 1. Background and motivation } & 1\end{array}$

2. The echo and sound concentration: a historical introduction 3

2.1 Ancient period 3

2.2 The seventeenth century 4

$\begin{array}{ll}2.3 \text { Eighteenth century and thereafter } & 7\end{array}$

3. Perception of reflections 9

3.1 Introduction 9

3.2 Research by Haas $\quad 10$

3.3 Threshold of detectability by Makrinenko 12

3.4 The method of Yamamoto 13

3.5 The Echocriterion by Dietsch and Kraak 14

3.6 Echo in reverberant fields $\quad 16$

4. Previous research and outline of the dissertation 17

4.1 Previous research on sound reflection from curved surfaces $\quad 17$

4.2 Outline of the dissertation $\quad 18$

\section{THEORY OF SOUND CONCENTRATION}

5. Geometrical acoustics 21

5.1 Thin lens theory $\quad 22$

$\begin{array}{ll}5.2 \text { Geometrical sound field } & 24\end{array}$

5.2.1 Spherical surfaces 24

5.2.2 Cylindrical surfaces $\quad 25$

5.2.3 Elliptical surfaces $\quad 27$

5.2.4 Other shapes 28

5.3 Geometrical computer models $\quad 29$

5.3.1 Image Source Method $\quad 30$

5.3.2 Ray Tracing 34

5.3.3 Beam Tracing 37

5.3.4 Overview of geometrical methods 38

6. Wave based methods 39

6.1 The Huygens-Fresnel Principle $\quad 39$

6.2 The Kirchhoff integral $\quad 40$

6.3 The exterior problem 44

6.4 Reflection and scattering $\quad 46$

6.5 Numerical calculations $\quad 48$

III. APPLICATION OF THE THEORY ON DIFFERENT GEOMETRICAL SHAPES

7. The sound field in a sphere 49

7.1 Integral formulation for a sphere $\quad 49$

7.2 Pressure in the focussing point of a sphere 51

7.3 Pressure in the far field for small aperture angle 53

7.4 Approximation of the sound field in a sphere 55

7.4.1 Approximate solution of the integral 55

7.4.2 Pressure in the focussing point based on the approximation 59

7.4.3 Pressure at the axis of the sphere segment 62 
7.4.4 Pressure in the focussing plane 68

$\begin{array}{ll}7.4 .5 \text { Pressure in the far field } & 72\end{array}$

$\begin{array}{ll}7.5 \text { Diffuse reflections in a sphere } & 76\end{array}$

$\begin{array}{ll}\text { 7.6 Conclusions on the sound field in a sphere } & 77\end{array}$

8. The sound field in a cylinder $\quad 79$

$\begin{array}{ll}8.1 \text { Integral formulation for a cylinder } & 79\end{array}$

8.2 Pressure at the origin of a cylinder $\quad 80$

8.2.1 Pressure at the origin of a circular cylinder 80

8.2.2 Pressure at the focal point of an elliptical cylinder 82

8.3 Approximate solution of the sound field in a circular cylinder 83

8.3.1 Approximate solution of the integral for a cylinder 83

8.3.2 Pressure in the origin based on the approximation 84

8.3.3 Pressure at the axis of the cylinder 86

8.3.4 Pressure in the radial plane of the cylinder 87

8.4 Diffuse reflections in a cylinder 88

9. The sound field in an ellipsoid 89

9.1 Geometry of the ellipsoid $\quad 89$

9.2 Pressure in the focal point of an ellipsoid 90

9.3 Example for the sound pressure field in an ellipsoid 91

10. The sound field from a double curved rectangular surface 93

10.1 Integral formulation for the double curved surface 93

10.2 Pressure in the centre of the double curved surface 93

10.3 Approximate solution for the double curved surface 94

IV. VERIFICATION OF THE KIRCHHOFF INTEGRAL

11. Experiments 99

11.1 Measurement method $\quad 99$

$\begin{array}{lr}\text { 11.1.1 Sound source } & 99\end{array}$

11.1.2. Microphone 100

11.1.3. Measuring system 102

$\begin{array}{ll}11.2 \text { Ellipsoidal reflector } & 103\end{array}$

11.3 Numerical approximation 104

$\begin{array}{ll}11.4 \text { Measurement results } & 104\end{array}$

$\begin{array}{ll}11.5 \text { Measurement accuracy } & 106\end{array}$

11.6 Conclusion 106

V. A PRACTICAL APPROACH ON ESTIMATION OF SOUND CONCENTRATION

12. Engineering approach 107

12.1 Geometrical sound field vs wave field approach 107

$\begin{array}{ll}12.2 \text { Geometrical approach } & 110\end{array}$

$\begin{array}{ll}12.3 \text { Wave field approach } & 111\end{array}$

12.3.1 Pressure at the focussing point 111

12.3.2 Pressure in the focussing plane 114

12.3.3 Pressure along the specular line $\quad 115$

12.3.4 Calculation examples 116

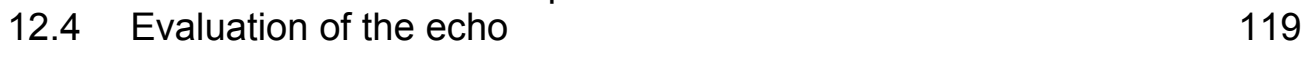

13. Reduction of reflected energy 121

13.1 Sound diffusion 121

13.2 Sound absorption $\quad 126$

13.3 Acoustically transparent boundary 130

13.4 Redirection of reflections 131 
14. Cases of sound concentration 133

14.1 Some cases from the literature 133

14.1.1 Salt Lake City Tabernacle 134

14.1.2 Small theatre with cylindrical rear wall 135

14.1.3 Haus des Lehrers, Berlin 136

14.1.4 Plenarsaal des Bundestags Bonn 137

14.1.5 Wigmore Hall Londen 138

14.2 Scale model research 142

14.3 Tonhalle Düsseldorf 146

14.3.1 Geometry 146

14.3.2 Approximation based on the basic geometry 147

14.3.3 Numerical approximation (before renovation) 148

14.3.4 Measurements in the hall before renovation 150

14.3.5 First proposed solution to solve the problem: diffusion 151

14.3.6 Scale model research of the first proposal: diffusion $\quad 152$

14.3.7 Second proposal: redirecting the sound 153

14.3.8 Scale model results with redirection of sound 154

14.3.9 Measurement results after renovation 155

14.3.10 Conclusions $\quad 155$

14.4 Royal Albert Hall Londen 157

14.4.1 Geometrical approximation $\quad 157$

14.4.2 Numerical approximation $\quad 161$

14.4.3 Scale model measurements 161

14.4.4 Real scale measurements $\quad 165$

14.4.5 Conclusions 165

14.5 The "Klankkaatser" 166

14.5.1 Approximated sound level in the focal point. 166

14.5.2 Numerical approximation 167

14.5.3 Measurements 168

$\begin{array}{lll}14.6 & \text { Office building with curved façade } & 170\end{array}$

14.6.1 Ray tracing model 171

14.6.2 Image Source Method (ISM) 172

14.6.3 Wave field approximation. $\quad 172$

14.6.4 Numerical approximation 173

14.6.5 Measurements 173

$\begin{array}{lll}14.7 & \text { Ellipsoidal meeting room } & 175\end{array}$

14.7.1 Geometry 175

14.7.2 Wave field approximation. 176

14.7.3 Numerical approximation 176

14.7.4 Measurements 176

VI CONCLUSIONS

15. Conclusions 179

15.1 Results of the dissertation 179

15.2 Recommendations for further research and developments $\quad 181$

$\begin{array}{ll}\text { References } & 183\end{array}$

$\begin{array}{lr}\text { Appendices } & 189\end{array}$

1. list of symbols 189

2. Curriculum Vitae 191

3. List of publications 192

$\begin{array}{ll}\text { Dankwoord } & 195\end{array}$ 



\section{SECTION I. INTRODUCTION INTO SOUND CONCENTRATION}

\section{Chapter 1 Background and motivation}

Many spaces have curved walls or ceilings. When sound is reflected by these curved surfaces the reflected sound field will converge and the sound level will be increased. When the reflected sound is concentrated in a narrow area the sound reflection may be strong enough to be audible as amplification, tone coloration or as a separate echo. An echo is an interesting acoustical phenomenon, which was already described in ancient literature (see chapter 2).

Curved surfaces may also generate multiple reflections. In figure 1.1 some effects of reflections from curved surfaces are shown. Figure 1.1b shows that the combination of a curved surface and a flat surface generates flutter echoes. The audible repetition rate depends on the source position. At the center all reflections will be heard; away from the center the reflections will move away and then back again (see e.g., [Cremer78] and [Moreno81]).

Another well known phenomenon is the whispering gallery effect (figure 1.1c), where multiple reflections move along the surface and maintain a bundle of sound. In this research we will focus on the single reflection by a curved surface (figure 1.1a).

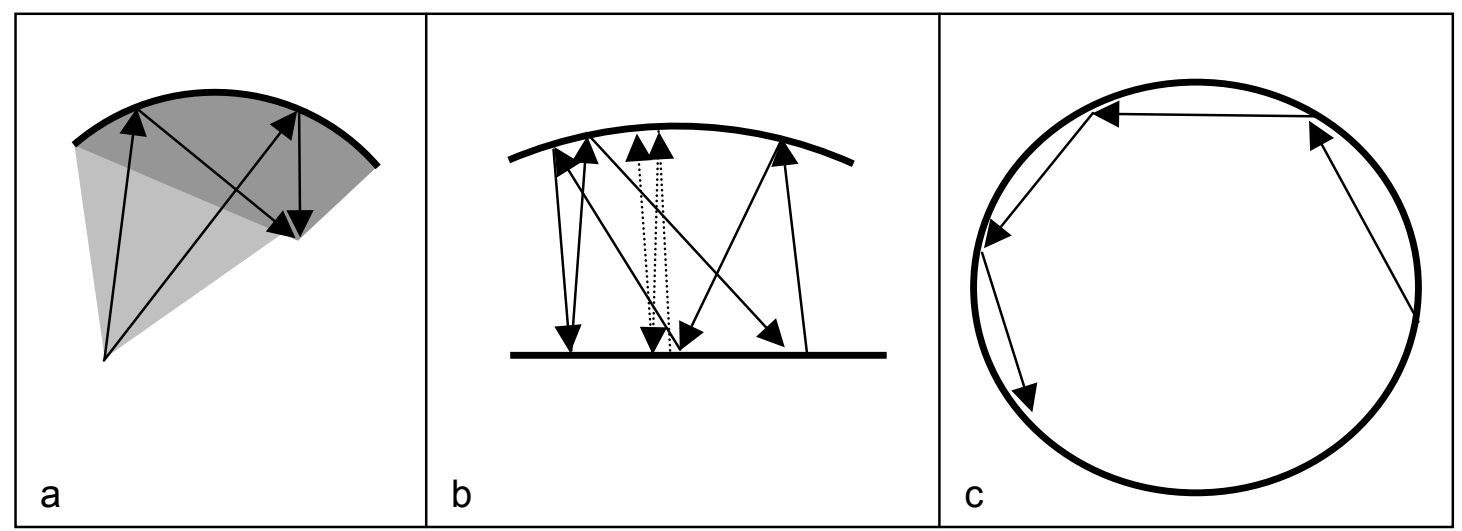

Figure 1.1. Reflections by curved surfaces. a: single concentrating sound reflection, $b$ : flutter echo caused by a curved and a flat surface, dotted: high repetition rate audible, solid: slow repetition rate at receiver position, c: whispering gallery.

Note: sometimes the definition of a whispering gallery also refers to single reflections from curved surfaces, e.g., in [Sabine22] the whispering gallery is defined as a room where faint sounds can be heard across extraordinary distances. This definition includes the single reflection.

The enhancement of reflected sound by concave surfaces can be acceptable or even advantageous for specific purposes such as parabolic reflectors above pulpits [Cremer78] or concave ceilings for performing music in small spaces (e.g., the London Wigmore hall for chamber music is appreciated for its strong sound, see par. 14.1.5). In larger spaces, with listeners further from the source, concave surfaces will lead to strong late reflections that will be heard as a disturbing echo. Especially in spaces with double curved surfaces these echoes are very persistent and although it can be done, it is difficult to get rid of them (as was done with the help of scale model 
research for the Royal Albert Hall and the Tonhalle Düsseldorf, as described in chapter 14).

In most practical situations, such as in smaller spaces, sound concentration causes problems in speech intelligibility, music appreciation, source localisation (e.g., [Okano01]) or feedback on microphones (e.g., [Fasold94]). With improved building technology, such as the use of new materials, laser-measuring techniques and CAD design tools, and new fashions in architecture (blobs), there are an increasing number of these problems. Solving these problems is the task of the acoustical engineer. Understanding the sound field caused by concave surfaces would allow innovative use of these geometrical designs to avoid acoustical problems.

Many authors of standard books on acoustics (e.g., [Bagenal31]), point out the danger of concave surfaces and describe the construction of the focusing point, the visualization of the wave front of the reflected wave (figure 5.1) or even provide a geometrical estimation method for the sound pressure due to sound concentration [Kuttrufff73]. They do not however give an estimation or calculation method for the sound pressure in the focusing point and its surrounding area.

In practical applications of room acoustics these curved surfaces will be calculated with geometrical methods, mostly by computer models using Image Source Method or Ray Tracing, in which the structure is modeled by flat segments. These methods however are based on energy addition. In the case of focusing, caused, for example, by a spherical or elliptical surface, the propagation distances of all the sound paths are equal and so is the phase. In that case the amplitudes have to be added, instead of energy.

Obviously there is a need for a better calculation method to estimate the sound pressure in the focusing point to be able to evaluate this sound pressure and, if necessary, alter the design or add sound reduction measures.

In this first section of the study a short introduction will be given of the historical context of the echo phenomenon (chapter 2).

Chapter 3 presents a summary of the relevant knowledge regarding psychoacoustical effects of sound reflections.

An overview of the research related to the sound concentration caused by curved surfaces will be given in chapter 4 .

In chapter 4.2 an outline of this study will be given.

If the reader wants to skip most of the theory, he may start at section $\mathrm{V}$, which presents a practical approach to the estimation of sound concentration. 


\section{Chapter 2}

\section{The echo and sound concentration: a historical introduction}

Echo can neither stay silent when spoken to, nor speak first by her own efforts

The word echo originates from the Greek verb "re-sound" $(\eta \chi \varepsilon \omega)$ and the mythical mountain nymph named Echo $(\mathrm{H} \chi \omega)$. There are several versions of this myth. The nymph Echo was devoted to mountains and caves. She was condemned by the goddess Hera for her incessant talking and singing. The punishment was that she could only repeat the last syllable of words that were spoken before her.

Because of her punishment and her inability to declare her love to Narcissus, she hid her sorrow in solitary caverns. Out of sadness her bones turned to stone and only her voice remained.

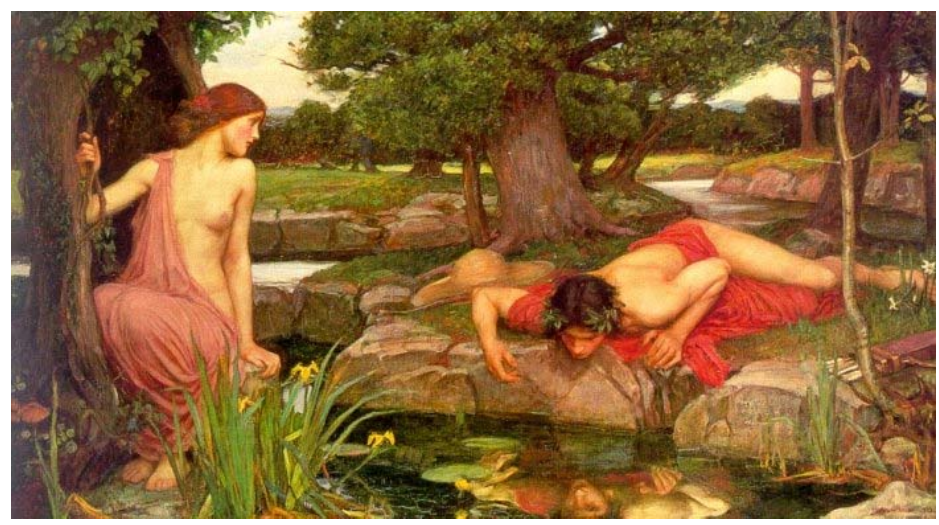

Figure 2.1. Painting of Echo and Narcissus by John William Waterhouse, 1903

\subsection{Ancient period}

Even in ancient times echoes were heard in nature, from mountains or large caves. And in this period these acoustical effects must have been an intriguing phenomenon. Without an easy to understand explanation, it was given a mythical cause.

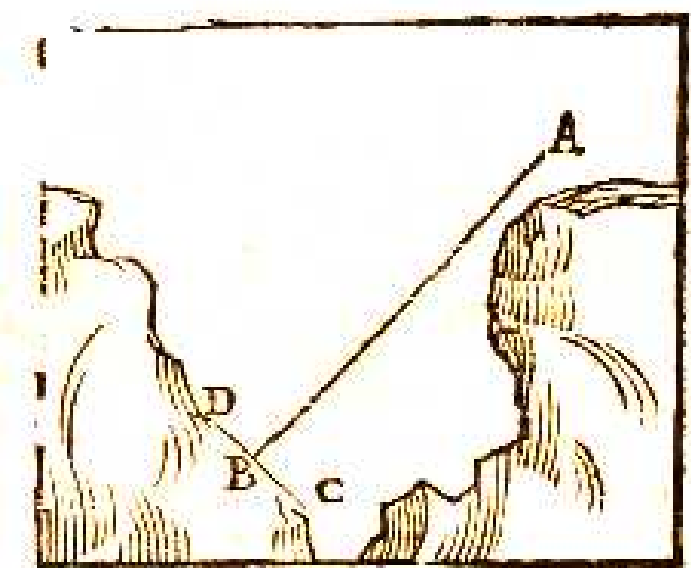

Figure 2.2. Illustration of a mountain echo: the sound from the source in $A$ is incident on the rather flat rock at $B$ and reflected back to $A$, from [Blancanus1620] 
The attention for acoustical phenomena in general during the ancient period can be found in Vitruvius' books on architecture. In his book he describes the purpose of vases in theatres, which were used to enhance the resonance [Vitruvius].

Acoustical vases can also be found in medieval churches (e.g., [Crunelle93]).

In many large, circular buildings or places outside, erected during the ancient period or in the Middle Ages, echoes can be heard. Some of the structures are famous for the whispering gallery effect. The first explanations of the phenomenon date back to the seventeenth century.

\subsection{The seventeenth century}

The concept of geometrical acoustics, where sound is treated as light, dates from the $16^{\text {th }}$ century (Ausonio, Della Porta [Tronchin08]). The wave propagation is illustrated with rays.

In the book "Spaera mundi", the Jesuit Josephus Blancanus wrote about the echo phenomenon. In the third chapter, "Echometria, idest Geometrica tractatio de Echo" 13 pages are devoted to a geometrical treatment of the echo phenomenon. For this study, this chapter has been translated to English [Blancanus1620].

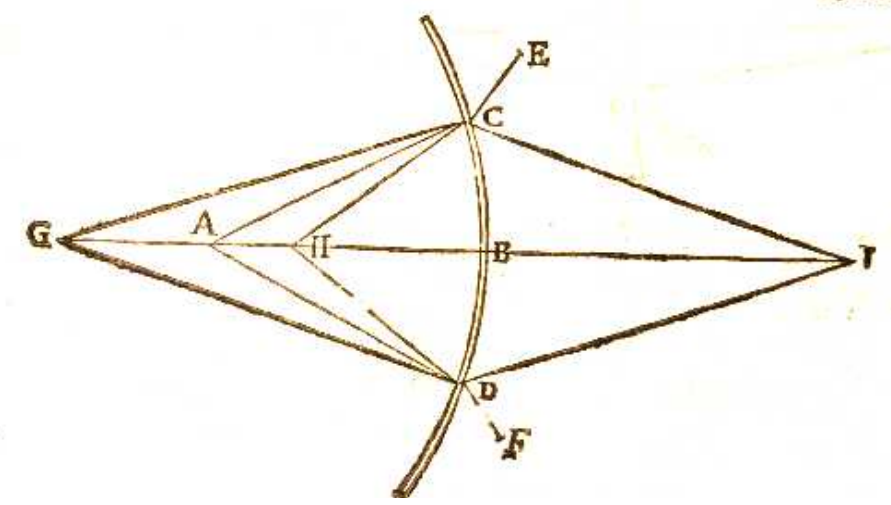

Figure 2.3. Geometrical explanation of the focusing effect by curved surfaces in [Blancanus1620]

The occurrence of echoes is described as the result of the reflection of sound from surfaces. Further, the amplification of sound through the focusing effect of concave surfaces is described (see figure 2.3).

The German Jesuit and scientist Athanasius Kircher published in 1650 the "Musurgia Universalis", [Kircher1650] in which he also paid attention to the acoustics of rooms (71 pages under the title: "Magia Phonocamptica", later published as a separate and extended treatise "Phonurgia nova sive conjugium mechanico-physicum artis \& naturae paranympha phonosophia concinnatum") .

In this book Kircher explains and illustrates the effects that elliptical forms have on the sound field, see figure 2.4. Kircher regarded the amplification of the sound from one focusing point to the other caused by elliptical vaults as an effective measure to increase speech intelligibility.

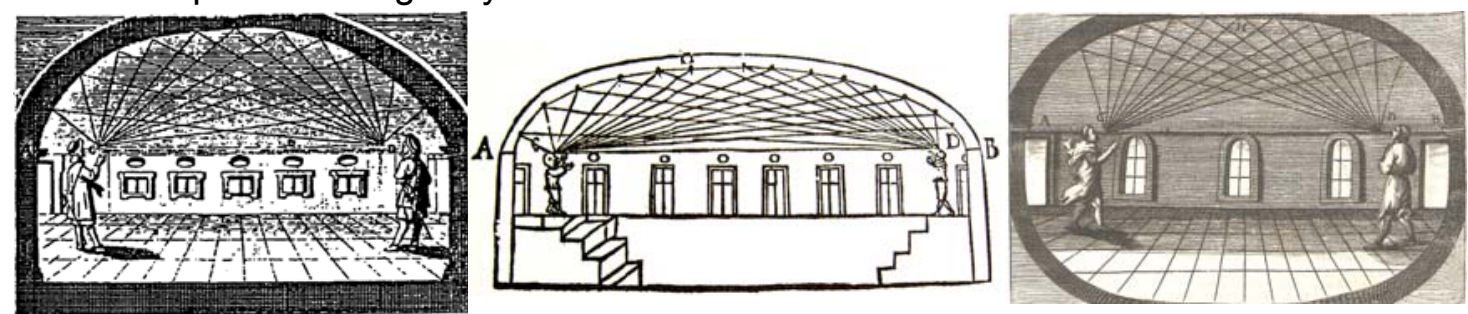

Figure 2.4. Three versions of the illustration of the focusing due to vaults in the Musurgia Universalis [Kircher1650] 
Kircher also described some case studies of echoes. One of them is a strong echo in a room with a dome shaped ceiling in the Palace of Heidelberg, see figure 2.6. However, Kircher was not able to give the correct explanation for this echo; he blamed it on the properties of the floor [Trochin08].

Note: Kircher worked and published this book in Rome. 1500 prints were circulated. On the occasion of the election of a new prior, the book was distributed among 300 Jesuits who were travelling home to places all over the world, thus distributing the book. One of the book's most famous pictures is that of the singing cock, see figure 2.5. The book was well known and it inspired composers such as J.S. Bach and J. Ph. Rameau ("La Poule"). Thus it is very likely that the book was also known by architects

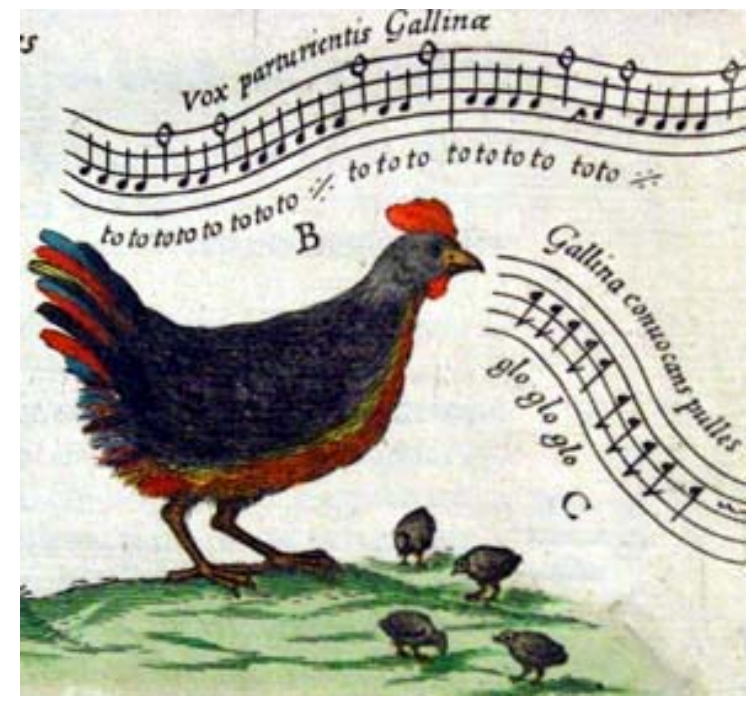

Figure 2.5. The singing cock, from [Kircher1650]

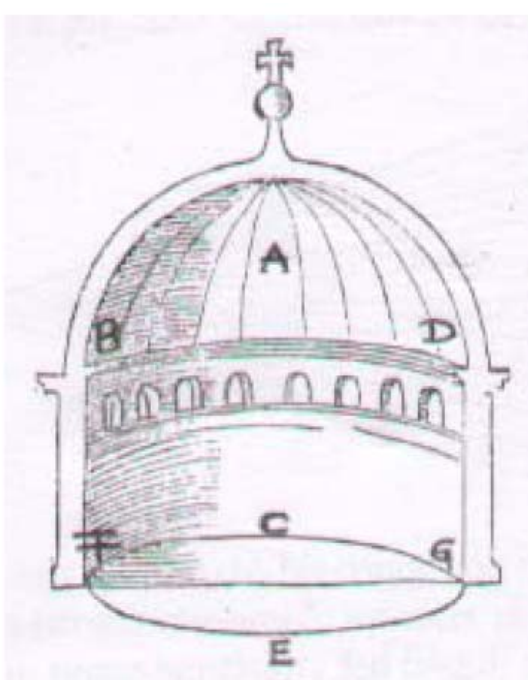

Figure 2.6. Room with echo, Palace Heidelberg, from [Kicher1620]

Another case study presented by Kircher is the flutter echo of the Villa Simonetta in Milan ("Villa of the echo"). On the second floor of this U-shaped villa there are two smooth and parallel walls. Between these walls a repetitive echo is heard when a sound is produced at a small window in one of these walls. Kircher gives the correct explanation and demonstrates this in a drawing (see [Trochin08]).

The publications of Blancanus and Kirchner show that by the middle of the 17th century some knowledge had been spread regarding the acoustical effects of curved surfaces.

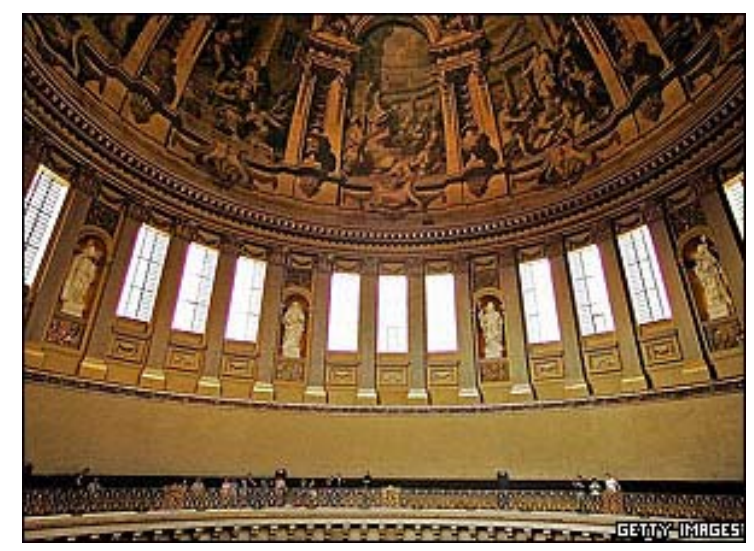

Figure 2.7. The Whispering Gallery in St. Paul's Cathedral, London 
Nowadays, the word echo refers to audible separate reflections that may be unwanted since it may disturb speech intelligibility or music perception. The general impression might be that builders of the seventeenth century were not aware of the echo effects that concave shaped rooms would introduce. However, this impression might not always be entirely correct. At that time, the sound effect was also considered an additional feature, contributing to the special character of the building. This can be deduced, for example, from the French dictionary of Trévoux [Trévoux1743], which was written less than a century later (1743). Here the echo was described as reflecting sound and in its architectural meaning was described as caused by certain vault shapes that form elliptical or parabolic surfaces, which double the sound and create artificial echoes.

As for the way to create artificial echoes Trevoux refers to Blancanus' chapter on Echometria. Further, the word Echometria is defined as being the science or art of creating echoes; to build structures whose disposition, especially of its vaults, forms echoes.

Therefore, because of the knowledge available that time, the special acoustical character of buildings from the seventeenth century, such as the cupola of St. Paul's Cathedral in London (see figure 2.7), might not be completely accidental.

Note: One building from the $17^{\text {th }}$ century is well known for its special sound effect: Saint Paul's Cathedral in London with its whispering gallery.

In 1673 Sir Christopher Wren started designing St. Paul's Cathedral. Wren was also a scientist, a professor of astronomy. He was also interested in acoustics [Jardine03]. Although the whispering effect of the gallery was probably not intended as such, the peculiar ("divine") acoustic of dome shapes must have been known to Wren, e.g., from [Blancanus1620] and/or [Kirchner1650].

The cause of the acoustical effect had long been a subject of scientific discussion:

According to George Airy, the Royal Astronomer, the whispering effect had to be caused by reflection from the dome [Airy1871]. This was corrected by [Rayleigh1896], who described the creeping of sound horizontally along the gallery. In its turn, this theory was corrected by [Raman21]. Differences between Raman and Rayleigh are explained by [Bate38].

From this discussion the distinction between the single focusing reflection and the whispering gallery, with its bundling of multiple reflections, has been made clear.

Elliptical or circular structures might deliberately be designed in a way to create the special "divine" acoustical effect. There is no evidence that it was considered a failure and one can imagine that the enhancement of the sound in moderate sized rooms was especially perceived as a positive effect.

On a scientific level, an important step in the wave theory of optics (and sound) was made by Christiaan Huygens with the formulation of his theory that every point on a wavefront can be thought of as an emitter of secondary wavelets, together forming the successive wavefront [Huygens1678], (see chapter 6). 


\section{$\underline{2.3 \text { Eighteenth century and thereafter }}$}

In the eighteenth and nineteenth centuries there was increasing insight into the acoustic/optical effect of curved surfaces.

At the second half of the eighteenth century there were some publications on theater building by Dumont and Patte ([Dumont1766] and [Patte1782]) where elliptical ground plans are favored. Patte recommends using ellipse floor plans explicitly to enhance the low sound levels at the last rows in a theatre. Not everyone agreed; for example, Saunders states that this enhancement is not proven and he proposes a half circular ground shape to keep the audience at equal distances from the stage [Saunders1790].

In a treatise on acoustics, the German architect Langhans states that elliptical ground plans result in unwanted echo effects (see figure 2.8), especially if the size of the theater is large, since the sound will be heard as two separate signals. The delay of the reflected sound should be less than $1 / 9 \mathrm{~s}$ [Langhans1810].
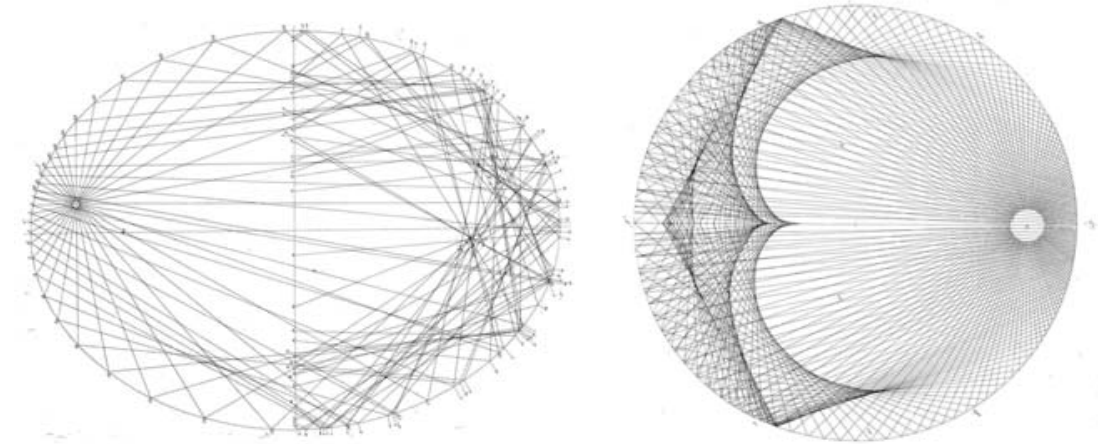

Figure 2.8. Visualization of the sound concentrating effects in elliptical or circular (theatre) rooms, by [Langhans1810]

Langhans states that one of the disadvantages of the sound concentration in elliptical theatres is that the high sound levels from a source in the focal point on stage mask the sound from other source positions on stage. As for the half circular halls proposed by Saunders, Langhans has the opinion that, due to the directional characteristic of the human voice, the audience in the proscenium (forestage) area should be much closer to the stage.

A notion of the influence of surface finishing was also present. Langhans states that sound concentration can be prevented by using diffusing finishing and objects.

Nevertheless, the ideas on elliptical ground plans found their way into theatre buildings. And even when they were not elliptical, due to this process of optimizing lines of sight and distance from the stage, most theatres have curved rear walls. There are even many theaters built in recent times that have curved rear walls and some of them suffer from strong sound concentration.

Regarding wave theory, it was Fresnel in 1818 who first applied Huygens' formulation to explain interference and diffraction. The mathematical basis was given by Kirchhoff in 1882 (see Chapter 6).

The increasing knowledge on acoustics in this period is accumulated in Lord Rayleigh's book on the Theory of Sound [Rayleigh1896]. Although he does not investigate this mathematically, Lord Rayleigh states in $\$ 285$ of this book that the Huygens principle may also be used for the reflections of sound from curved surfaces. This will be done in this study (chapter 6). 
Relevant developments in the $20^{\text {th }}$ century concerning sound reflections from curved surfaces will be discussed in paragraph 4.1.

One relevant issue in the $20^{\text {th }}$ century was the development of measurement methods, both regarding transducers (e.g., microphones) and signal processing.

The reaction of any dynamic system can be described with the impulse response, the output of the system when excited with an impulse as input signal. By knowing the output for an impulse, the output for any type of input signal can be determined.

To measure the impulse response an actual impulse sound source may be used, in room acoustics e.g., a balloon burst, a pistol shot or a spark. The disadvantage of these sound sources is that the signals are not an ideal impulse, the reproducibility is not always perfect and non-linearity's may occur in the system. The use of signal processing made it possible to use other input signals than impulses and calculating the input response (see e.g. [Rife89], [Bendat71]).

The impulse response enables the detection and evaluation of individual reflections in time domain. Figure 2.9 shows an example of an impulse response where the direct sound, after some delay corresponding to the traveled distance from source to receiver, and the reflections are clearly visible. The left graph is on a linear scale, the right on a decibel scale.
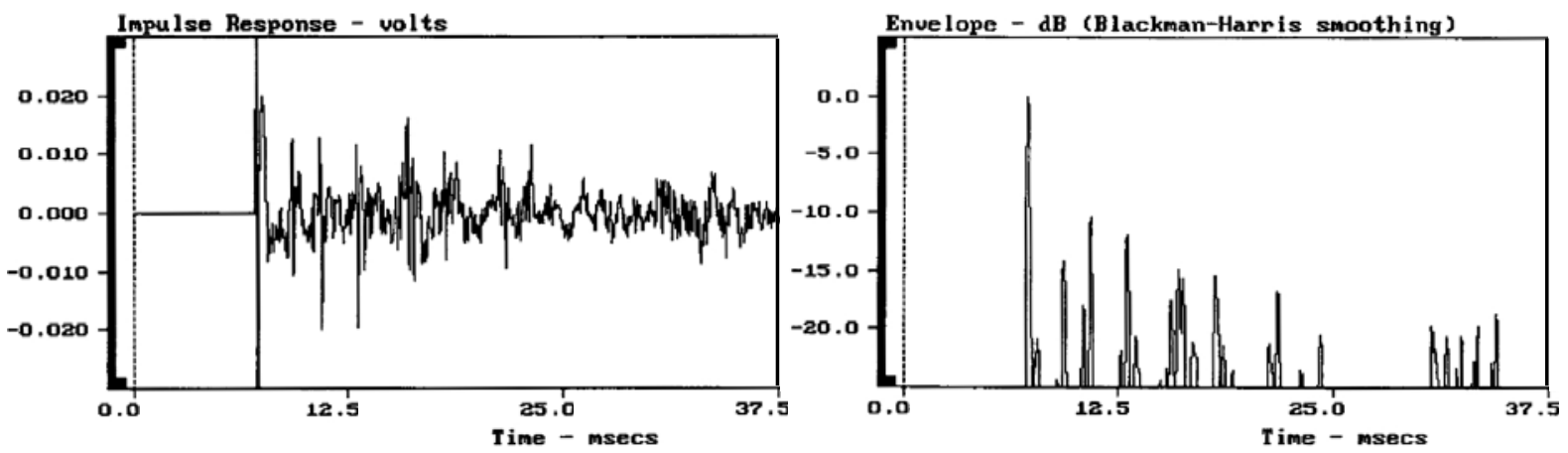

Figure 2.9. Example of an impulse response of a loudspeaker in a living room, showing direct sound and reflections as function of time, left: system output on linear scale, right: system output on a decibel scale, from [Rife89]. 


\section{Chapter 3. Perception of reflections}

Focusing is only relevant when it is in any way disturbing. To be able to evaluate sound concentration knowledge on perception is essential. In this chapter the knowledge concerning the disturbance caused by echoes will be summarized.

\section{$\underline{3.1 \text { Introduction }}$}

Adding one or more reflections to the direct sound will influence the perception of the sound. When the reflection arrives within a short delay after the direct sound some kind of coloration will be observed. This coloration can be perceived as an enrichment of the sound or it can impair the character of the sound.

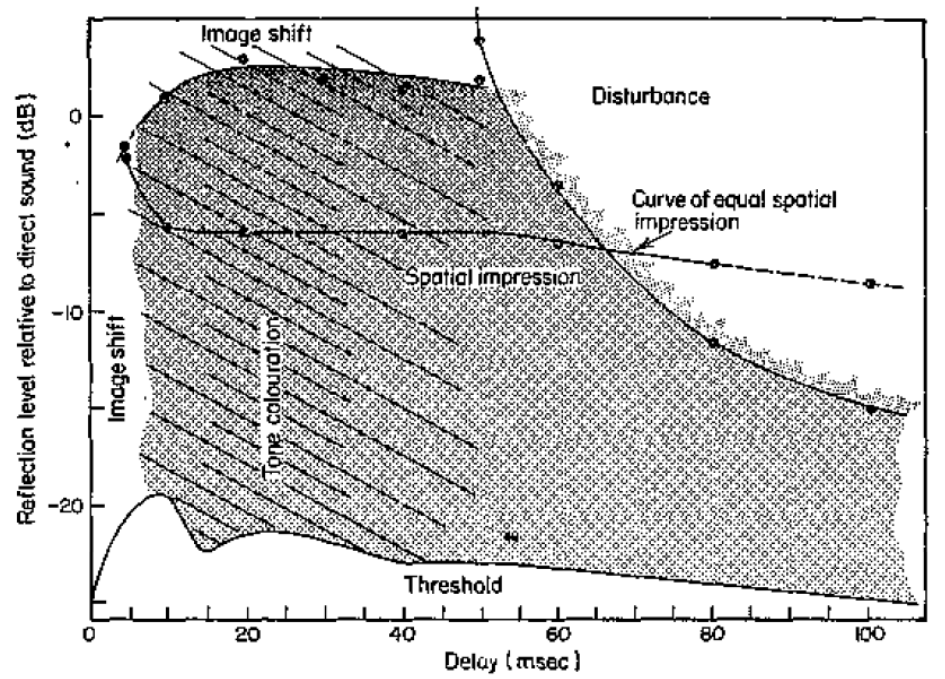

Figure 3.1. Subjective effects of a single side reflection $\left(a=40^{\circ}\right)$ of variable delay and level using music, from [Barron71].

The perceived effect of early reflections is shown in [Barron71] in figure 3.1. The $x-$ axis shows the time delay between direct sound and early reflection (total scale is 0,1 $\mathrm{s}$.). A delay of $100 \mathrm{~ms}$ corresponds to an (additionally) traveled distance of $34 \mathrm{~m}$. He found that the delay was relatively unimportant for reflections delayed between 10 and $60 \mathrm{~ms}$ relative to the direct sound. In this range a spatial impression is the positive effect of early reflections. Ceiling reflections did not produce the same effect, but only produced tone coloration. Delay of single side reflections proved to be the principle determinant of spatial impressions.

More adverse effects will occur when the delay of the reflected sound (relative to the arrival of the direct sound) increases. The reflection may be heard as an echo. The echo is defined as "any sound reflection that is subjectively noticeable as a temporal or spatially separated repetition of the original signal" [Kuttruff73].

This section will summarize some of the essential criteria for echo audibility.

For a long time it has been known that for fast speech a reflection can be heard separately if the delay is more than around 50 to $100 \mathrm{~ms}$ (e.g., in [Gehler1787] it is considered to be about $1 / 9 \mathrm{~s}$.). The first clear numerical value is given by [Petzold27]: $0.05 \pm 0.01 \mathrm{~s}$., corresponding to a distance difference of $17 \pm 3 \mathrm{~m}$ of the reflected sound. 
Whether a reflection is audible as an echo depends on:

- the time length of the delay,

- sound level of the reflection relative to the direct signal,

- the time structure of the source signal (impulse sound, speech, music),

- the frequency content of the signal,

- the direction of the signal and

- masking effects by other (intermediate) reflections

In the next sections some methods of evaluation of echo audibility will be summarized.

\subsection{Research by Haas}

A well known investigation of the influence of a single (artificial) echo as a function of a given set of parameters on the audibility of speech was published in [Haas51].

For average speech figure 3.2 shows the percentage of people that could hear a second signal as a separate sound, for different levels and time delays of the reflected sound relative to the direct sound.

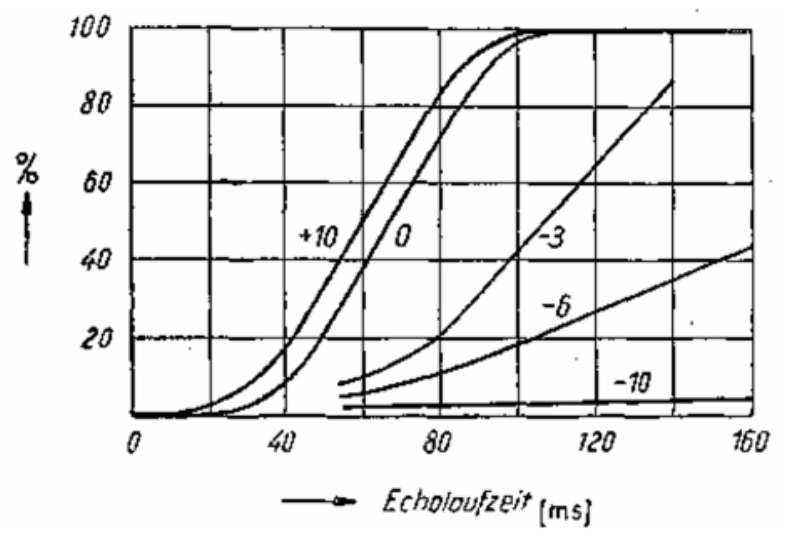

Figure 3.2. Percentage of listeners disturbed by a delayed speech signal. The relative echo levels (in $d B$ ) are indicated by numbers next to the curves [Haas51].

Haas indicates that, "An increase of the reflection strength by $10 \mathrm{~dB}$, for example by sound concentration due to curved surfaces of a cupola or planetary in the old building style, the critical delay difference is only slightly less than the value for equal strength. It reduces from 68 to $60 \mathrm{~ms}$ " [Haas51]. The critical delay difference refers to the time delay that listening to the speech is indicated disturbing by $50 \%$ of the listeners, for a given level difference between the two signals.

Within this $60 \mathrm{~ms}$ critical delay difference the reflected signal can be up to $10 \mathrm{~dB}$ louder than the direct signal without being disturbing. A time delay of $60 \mathrm{~ms}$ corresponds roughly with sound traveling a distance of $20 \mathrm{~m}$. As shown later, concave surfaces may increase the reflection strength by $10 \mathrm{~dB}$ or more. Thus with a concave surface at a distance of more than $10 \mathrm{~m}$ the reflection may be heard separately from the direct sound.

For longer delay times than the critical delay difference for $0 \mathrm{~dB}$ difference, the echo disturbance level is reduced with increasing time delay. For reflections with a level of $10 \mathrm{~dB}$ below the direct sound, no disturbance was found by [Haas51]; but a disturbance was found by others.

The perception of the echo depends on the frequency content. For high frequencies the critical delay difference is shorter (see figure 3.3 and par. 3.4). As shown in section 7.4.4. and illustrated in figure 12.4, the area where a low frequency echo will occur will be much larger than the area where the high frequency echo will occur. This means that in the larger area where the low frequency (part) of the echo 
dominates, the critical delay difference (for equal strength) increases to about 100 ms.

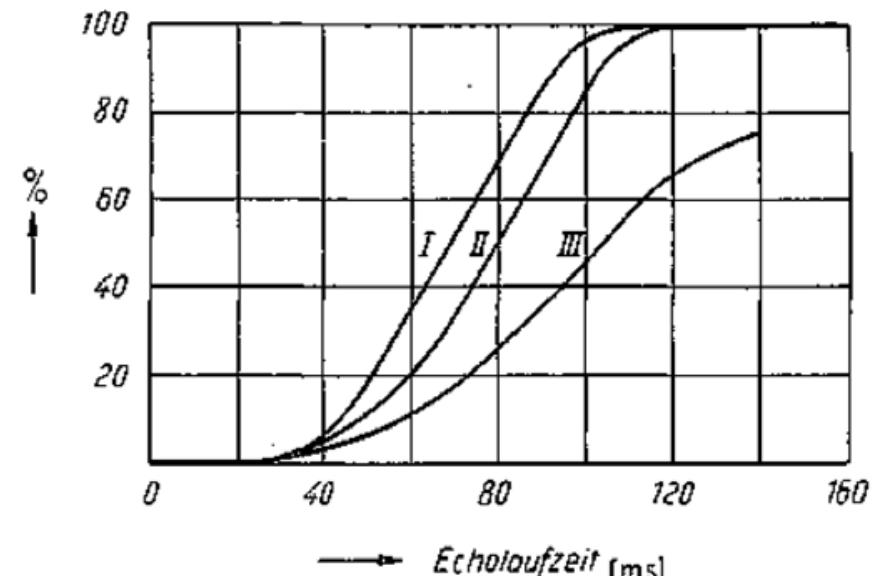

Figure 3.3. Percentage of listeners disturbed by a delayed speech signal for different frequency content of the signal ([Haas51].):

I. direct signal and reflection have the same spectrum

II. lower frequencies of the reflection are reduced (from $300 \mathrm{~Hz},-5 \mathrm{~dB}$ at $100 \mathrm{~Hz}$ )

III. higher frequencies of the reflection are reduced (from $1 \mathrm{kHz},-15 \mathrm{~dB}$ at $10 \mathrm{kHz}$ )

Further research was done and criteria were formulated in the same time period by [Bolt50], [Meyer52], [Muncey53], [Dubout58], [Lochner58], [Niese61] (see also par.3.3).

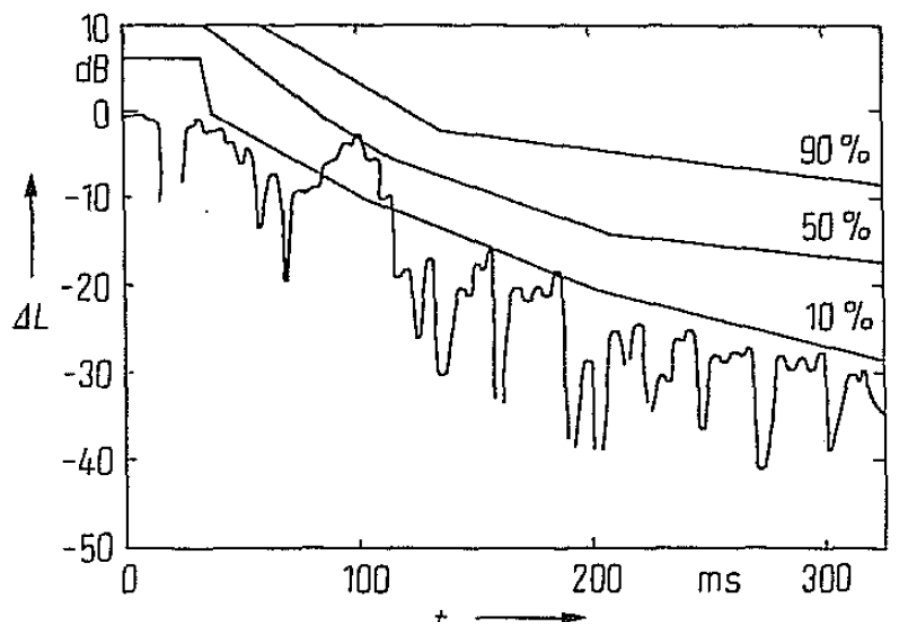

Figure 3.4. Example of an echo sound signal that is disturbing for $50 \%$ of the listeners. Contours of constant percent disturbance, echo sound level (relative to direct sound) as a function of delay time [Bolt50]

For example [Bolt50] formulated criteria based on the results of the dissertation of Haas in 1949 (see also [Haas51]), as shown in figure 3.4. 


\section{$\underline{3.3 \text { Threshold of detectability by Makrinenko }}$}

In [Makrinenko86] the threshold of detectability is given for various signals, based on measurement data of various authors, see figure 3.5. The threshold of echo detectability depends on the delay time between direct sound and the reflection $\Delta t$ (in $\mathrm{ms})$. The given threshold for speech can be approximated by:

$$
\Delta L=-38,4 \lg (\Delta t)+55,1
$$

The critical delay difference $(\Delta L=0 \mathrm{~dB})$ depends on characteristics of the signal such as frequency content and temporal behaviour. For speech, the critical delay difference is around $27 \mathrm{~ms}$, see figure 3.5.

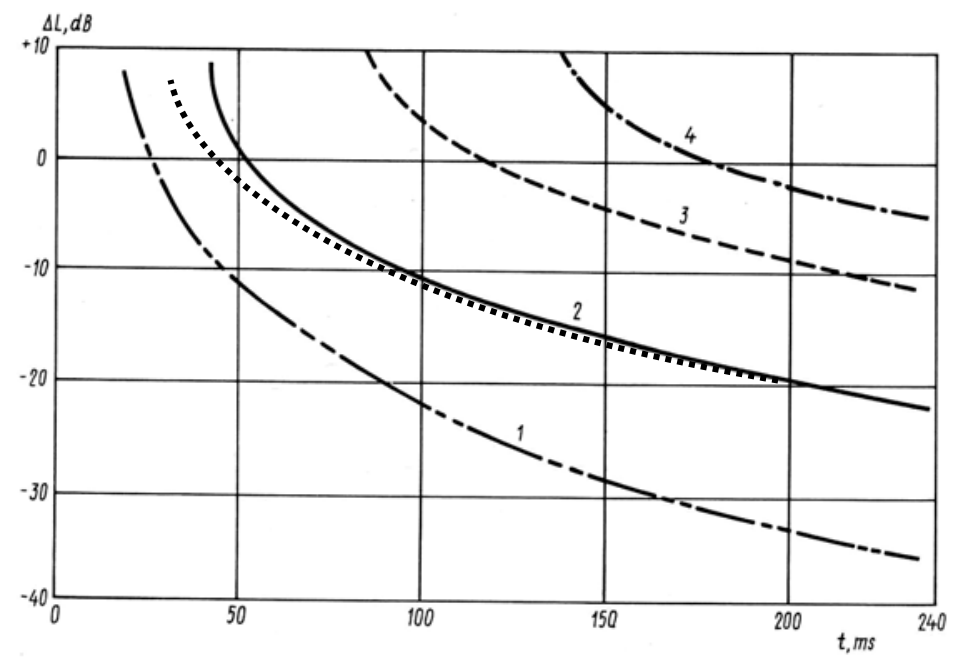

Figure 3.5. Threshold of detectability for various sound signals: 1. speech; 2. violin (fast passages); 3. organ; 4. violin (slow passages) [Makrinenko86];

Dotted line: Echo indicator for music according to [Dietsch86], formula (3.5)

Typical complaints about audible echoes with music in public performance spaces refer to echoes from impulsive instruments like percussion and piano (e.g., the "knocking ghost" in the Tonhalle Düsseldorf, see par. 14.3). It may therefore be misleading to use the curve for "violin (fast passages)" in case of spaces for musical performance. The curve for speech is more appropriate. 


\subsection{The method of Yamamoto}

The aforementioned research describes the effect of a single reflection. In real life situations the other reflections from room surfaces contribute to the overall listening experience and therefore influence the perception of the echo reflection.

In [Yamamoto71] an evaluation method for echo perception of multiple reflections from an impulse sound source (time duration not more than $10 \mathrm{~ms}$ ) is presented. With this method, masking curves are defined with the following characteristics:

- the critical delay difference is frequency dependent:
o $500 \mathrm{~Hz}$
$45 \mathrm{~ms}$
o $1000 \mathrm{~Hz}$
$35 \mathrm{~ms}$
o $2000 \mathrm{~Hz}$
$25 \mathrm{~ms}$

During this duration the masking curve is constant.

- After this delay the masking curve falls off, with a drop rate $\delta$ depending on the sound level: $\delta=L / 300[\mathrm{~dB} / \mathrm{ms}]$, where $L=$ level of the direct sound or a reflection.

- When a reflection exceeds this masking curve, this excess will be determined: $\Delta L$ is the reflection level minus masking level.

- From this reflection in the same way a masking curve will be constructed.

- If the excess of the masking curve $\Delta L$ is less than the following values, the reflection is not considered to be disturbing:
o $500 \mathrm{~Hz}$
$\Delta L<3$ to $4 \mathrm{~dB}$
o $1000 \mathrm{~Hz}$
$\triangle L<2$ to $3 \mathrm{~dB}$
o $2000 \mathrm{~Hz}$
$\Delta L<1,5$ to $2,5 \mathrm{~dB}$

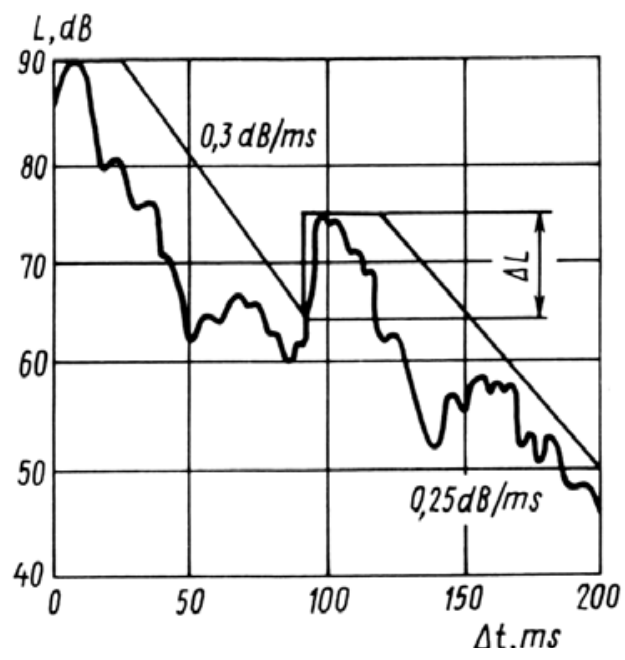

Figure 3.6. Echo evaluation method of multiple reflections by [Yamamoto71]

The method is illustrated in figure 3.6. The method suggests that it is possible to fill up the time between direct signal and reflection with reflections, in a way that neither of them will be heard as a separate echo, provided each of them will be below the evaluating values given above.

The applicability of this aspect, as well as its usefulness with other types of signals (e.g., speech, music), is unclear. 


\subsection{The Echocriterion by Dietsch and Kraak}

A more recent, but rather complicated, echo evaluation method based on the measured impulse response is given in [Dietsch86]. In this method the time dependent build up function of the center time of the impulse response $t_{s}(\tau)$ is defined as:

$$
t_{s}(\tau)=\frac{\int_{0}^{\tau} t|p(t)|^{n} d t}{\int_{0}^{\tau}|p(t)|^{n} d t}
$$

A strong reflection in the impulse response will cause a rather sudden shift in center time. Therefore, the alteration of the center time with time $\Delta t_{s}(\tau) / \Delta \tau$ at the moment of the reflection (see figure 3.7) is the echo indicator that is evaluated.
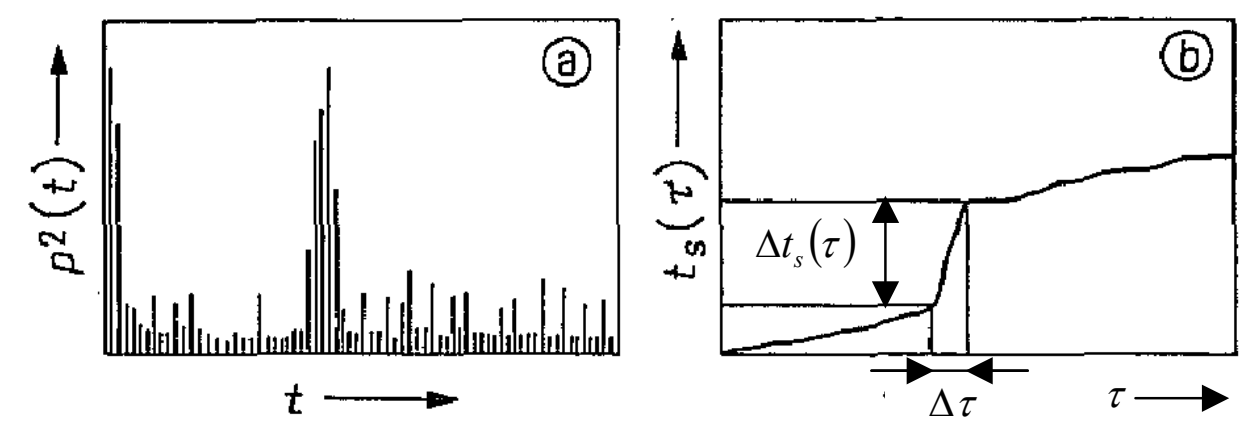

Figure 3.7. Example of an impulse response with an echo (a) and the corresponding build up of the center time as a function of time (b) [Dietsch86].

For the standardized center time $(\tau=\infty)$, the exponent $n$ in (3.2) equals 2 . In [Dietsch86] it was found that the correspondence of the build up function to the measurement data was optimal with $n=1$ for music and $n=2 / 3$ for speech. Furthermore, the optimal $\Delta \tau$ for evaluating the $\Delta t_{s}(\tau)$ was found to be $14 \mathrm{~ms}$ for (rhythmic) music and $9 \mathrm{~ms}$ for speech. For the echo indicator $\Delta t_{s}(\tau) / \Delta \tau$ (in [Dietsch86] called Echokriterium "EK") the values found are listed in table 3.1

Table 3.1 Values for the echo indicator $E K=\Delta t_{s}(\tau) / \Delta \tau$ found by [Dietsch86]

\begin{tabular}{|l|l|l|}
\hline & Music & Speech \\
\hline $\mathbf{1 0 \%}$ disturbed by the echo & 1,5 & 0,9 \\
\hline $\mathbf{5 0 \%}$ disturbed by the echo & 1,8 & 1,0 \\
\hline
\end{tabular}

An important advantage of this method is that the masking effect of energy before the disturbing echo reduces the echo indicator. The method also takes into account the duration of the reflection.

Although this method is meant for evaluation of complex signals, it can be simplified for the case of just a primary (direct) signal $p_{d}$ and secondary (echo) signal $p_{e}$, both having the same time-structure, the difference is described by a delay $\Delta t$ and a level decrease $\Delta L$. For that case the center time shift $\Delta t_{s}$ will be:

$$
\Delta t_{s}=\frac{p_{e}^{n} \cdot \Delta t}{p_{e}^{n}+p_{d}^{n}}
$$


and

$$
\Delta L(\Delta t)=\frac{20}{n} \lg \frac{\Delta t_{s}}{\Delta t-\Delta t_{s}}
$$

Using the maximum center time shift $\Delta t_{s}$ based on table 3.1, formula (3.4) will result in the maximum SPL of the echo signal, as a function of delay time $\Delta t$.

For music, with $n=1$ and $\Delta \tau=14 \mathrm{~ms}$, the maximum $\Delta t_{s}(10 \%$ disturbed) will be $1,5 * 14=21 \mathrm{~ms}$. For this case the maximum echo level is described by:

$$
\Delta L(\Delta t)=20 \lg \frac{0,021}{\Delta t-0,021}
$$

For $\Delta t=42 \mathrm{~ms}, \Delta L=0 \mathrm{~dB}$. For longer delay times the maximum level drops, e.g., for $100 \mathrm{~ms} \Delta L=-11,5 \mathrm{~dB}$. This curve is rather close to the curve for echo detectability of fast violin by Makrinenko (see figure 3.5). 


\subsection{Echo in reverberant fields}

The methods of [Yamamoto71] and [Dietsch86] can be used to evaluate measurement results in the case of more complex signals in existing situations.

However, a tool is needed to evaluate individual echoes in the design stage, where a measurement of the signal is not possible. The criterion in formula (3.5), as well as the echo detectability method used by Makrinenko, assumes that no other signals are present. In reality though there are many reflections, of which a single, stronger reflection can produce an echo and the other reflections may mask the echo. So a rough estimation of the level of the decaying sound field needs to be known to enable the determination and evaluation of the level of the echo above that decaying sound field, e.g., with the method of Yamamoto.

A fist possibility is to assume the "masking" reflections to be reflecting off of a flat panel and fully reflective. The level of the individual reflections will be dependent on the traveled distance of the path from source to receiver. Assuming an omnidirectional source, the level of the reflection, compared to the direct sound will be:

$$
\Delta L_{r}=20 \lg r_{d} / d
$$

where $d: \quad$ traveled distance of the (multiple) reflected sound

$r_{d}$ : distance from the source to the receiver (direct sound)

The level difference $\Delta L_{r}$ will be the limiting case. Due to absorption and diffraction at surfaces the level decrease will be stronger, especially for later arrivals.

A second possibility, for geometrical sound field amplification, is to use the expressions presented in chapter 5 that directly yield the amplification due to the curvature of the reflecting surface $\left(\Delta L_{c}\right)$.

A third possibility is to estimate the level of the reverberation from ray tracing calculations as a reference for evaluating the echo.

Especially for later reflections, an evaluation of the echo relative to (3.6) or ray tracing will be more appropriate than the use of the echo detectability by Makrinenko or the Dietsch and Kraak method for a single reflection (formula 3.5). While the latter methods will be more appropriate if the echo to be evaluated is the first reflection in the room. 


\section{Chapter 4 \\ Previous research and outline of the dissertation}

\subsection{Previous research on sound reflection from curved surfaces}

Usually in room acoustics the sound field is predicted using geometrical acoustics. Geometrical methods are described in many handbooks, e.g., [Bagenal31], [Cremer78], [Kuttruff73], and [Makrinenko94].

Specifically for the converging sound field that is created by curved surfaces, algorithms can be derived, ,This has been done, for example, for cylindrical and spherical surfaces by [Rindel85](see also [Long06]) and [Kuttruff73]). The sound field can also be predicted for other shapes by following the rules of optics.

As shown in section 5.3, computer modelling based on geometrical acoustics is a rather limited tool for the prediction of reflection from curved surfaces. These techniques can be improved to some extent by using the right sized segments [Kuttruff93] or by using an exact (parameterised) shape [Mommertz95]. Outside the focusing point the accuracy can be improved by using coherent Beam Tracing [Jean07]. The essential deficiency of the geometrical methods is the (infinite) pressure in the focusing point. This problem cannot be solved satisfactory using geometrical methods.

Nevertheless, the geometrical method might give a sufficient prediction for room acoustical purposes (depending of the accuracy one wants to obtain), as long as it is only applied at a sufficient distance from the focusing point.

The sound pressure in the focusing point can only be calculated by incorporating the wave character into the calculation method.

The Huygens principle can be used for this purpose. In his book on the theory of sound, Rayleigh points out that the Huygens principle may also be used for the reflections of sound from curved surfaces, but from that point it is not further investigated mathematically [Rayleigh1896;§285]. Within the study of room acoustics there were very few later attempts made at calculating the reflected sound field from curved surfaces.

A wave theoretical description of the sound field by a parabolic reflector used as directional microphone is given in [Wahlström85].

One important paper on this topic comes from Kuttruff; in it he describes the sound field in a cylindrical space [Kuttruff93]. In a later paper [Yamada05], the sound field for different types of cylinders (circular, elliptical, parabolic and hyperbolic) is described.

A similar situation as for the reflection of sound can be found in ultrasonics, where focussed radiators are used. Focussed radiators are made of piezoelectric crystals, in the form of a thin spherically curved shell. With electric excitation the radiated sound pressure will concentrate in the centre. This method is used in acoustic microscopy. The pressure field generated by these radiators have been studied extensively (see e.g., [O’Neil49], [Lucas82], and [Chen93]). 


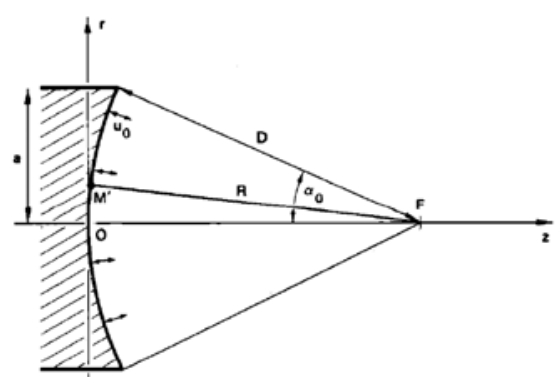

Figure 4.1. the focusing radiator [Lucas82]

The phase at the radiator surface is uniform, corresponding to the reflection of sound from a source in the centre of a sphere segment. The radiators used however, have a small aperture angle. The mathematical methods developed for the solution of this problem are an important basis for the work presented here. Limitations of the description of the sound field based on these radiators is the equal phase at the radiator surface (in room acoustics this means the source in the centre) and the relatively small aperture angle of the sphere segment.

Numeric computer programs that fully incorporate the wave character, such as Boundary Element Method (BEM) and Finite Element Method (FEM) or Finitedifference time-domain (FDTD) can be applied to calculate the sound field in a room. These calculations however are extremely time-consuming, in modeling and calculation time, and therefore are not yet practicable on the scale of a (mostly complex) room (see e.g., [Yokota04])

On a smaller scale, for example for the scattering behavior of diffusers, these methods can be applied (e.g., [Cox04]).

\subsection{Outline of the dissertation}

Obviously there is a need for a better calculation method to estimate the sound pressure in and around the focusing point to be able to evaluate this sound pressure and, if necessary, alter the design of the room or add sound reduction measures.

The primary purpose of this dissertation is to gain a better understanding and estimation of the sound pressure level due to reflections from curved surfaces, especially spherical surfaces, both in and around the focusing area.

An engineering method has to be developed to be used as a practical tool in room acoustic design.

In Section II the basic theory of sound concentration is presented.

The application of geometrical methods for reflections from curved surfaces is described in chapter 5. Further, the results are described (par. 5.3) of a study concerning the possibilities of applying geometrical methods for calculating sound concentration. It is concluded that geometrical methods may be used to calculate the converging sound field, but not in and around the focusing point.

Focusing can only be calculated with wave-based methods. Chapter 6 explains the wave extrapolation method based on the Kirchhoff Integral Equation, which is the basis for the approximations in section III.

In section III, this wave theory is applied to the sound reflection from differently curved shapes. In chapter 7 the theory is further developed for spherically curved surfaces. This is the main part of this dissertation. 
An analytical solution is presented for determining the sound pressure in the focusing point.

For the sound field outside the focusing point, there is no closed form analytical solution available from the Kirchhoff Integral Equation. Approximation techniques used in optics and ultrasonics are extended for application to sphere segments with a larger aperture angle and source positions outside the centre.

Chapter 8 describes the results found in the literature on the sound concentration of cylindrical shapes. The theory is extended for sources and receivers outside the centre line.

For the ellipsoid shapes described in chapter 9, no analytical solutions are available but some numerical results are presented.

In chapter 10 the results are presented of the analytical description of the reflection from a rectangular, double curved surface. For this geometry a closed form solution was derived using error functions.

In section IV the results of a verification of the Kirchhoff Integral Equation are given. Chapter 11 describes the measurement results on a half ellipsoid shape. The results correspond to the calculation results.

Section $V$ is dedicated to the practical aspects of the mathematical results of section III. In chapter 12 the formulas to be used in practical situations are summarized. The methods that can be used to reduce focusing without completely changing the geometry of the room are described in chapter 13.

An important conclusion is that using absorption or diffusers is generally not sufficient to reduce the strong focusing caused by spherical reflectors.

In addition to the traditional absorption and diffusion methods, the redirecting of energy is added as a sound reduction technique that reduces or eliminates the focusing.

Chapter 14 describes some cases of sound concentration. In a number of these cases it was possible to do measurements, enabling a comparison with the calculation results. In practical cases it is important to be able to evaluate if an echo is tolerable or not.

Section VI contains the conclusions of this dissertation, as well as discussion and recommendations for further research.

Note:

In this study the point or area where focusing occurs will mostly be referred to as the "focusing point" or "focus" and "focusing area".

Generally in optics the word "focal point" indicates the focusing point in the special case of a known source. It refers to:

- spherical reflectors:

- circular cylinders:

the source at infinite distance, on the axis.

- elliptical cylinders: the source in the centre axis,

the source in one focal axis and the receiver in the other focal axis, at the same height.

- ellipsoid shapes: the source in one focal point, the receiver in the other. 


\section{SECTION II. THEORY OF SOUND CONCENTRATION}

In this section the theory that can be applied to calculate the sound concentration due to reflections against curved surfaces is described. Chapter 5 evaluates the geometrical methods and chapter 6 evaluates the wave-based Kirchhoff Integral method.

\section{Chapter 5}

\section{Geometrical acoustics}

In geometrical acoustics the behavior of the sound is described with geometrical rules, as in geometrical optics. Interference, diffraction and bending of sound waves are not considered. The propagation of sound waves is represented by rays. These rays have a propagation direction normal to the wavefront and can therefore be considered as small portions of a spherical wave. A spherical wave has many rays.

The rays reflect according to Snel's law at the intersection point with the reflecting surface.

Note: Snellius was the latin name of the Dutch mathematician and physicist Willebrord Snel van Royen (1580-1625).

For concave surfaces the propagation of the sound wave can be constructed from the (multiple) image sources, mirrored using the tangential planes at the intersection points as shown in figure 5.1 .

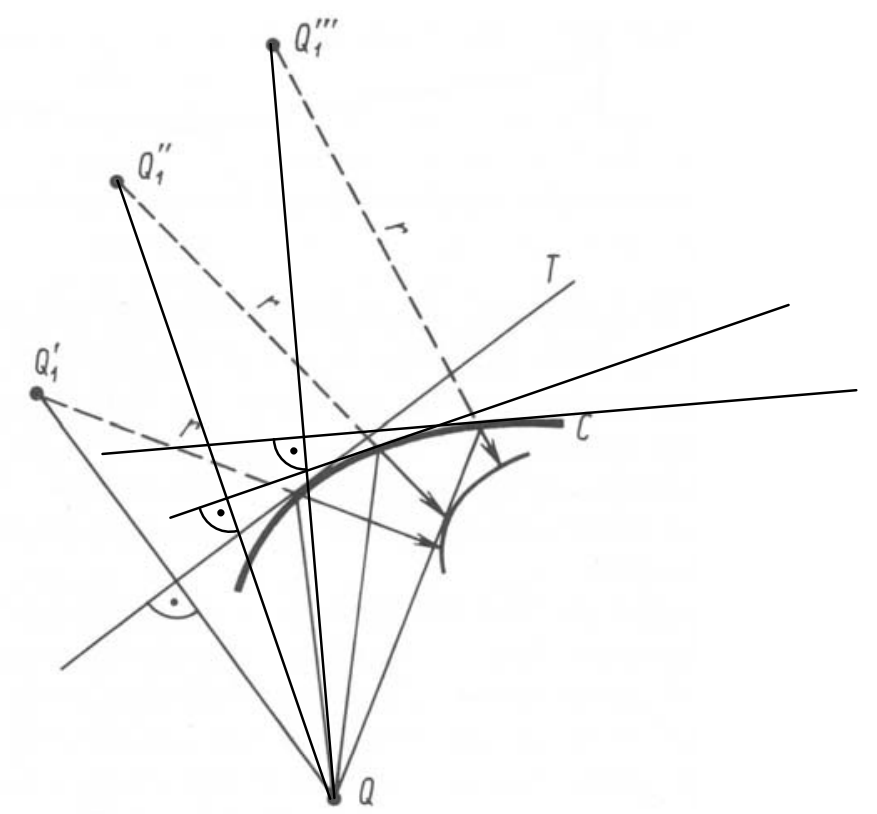

Figure 5.1. Construction of the wavefront reflected from a concave surface [Makrinenko86]

Applications of geometrical acoustics with curved surfaces include parabolic pulpit reflectors (e.g., [Cremer78]) and convex reflectors in theaters and concert halls.

The concept of geometrical acoustics in only valid for high frequencies, where the dimensions of the reflecting surfaces are considerably larger than the wavelength.

But the distance to the reflecting surface is also relevant. From the construction of Fresnel zones the distance can be determined where the geometrical reflection will 
change into a spherical wave propagation (originating from the reflecting surface). For a plane incident wave on a flat disk with radius $R$ this will be [Cremer78]:

$$
d_{\text {geo }}=\frac{2 R^{2}}{\lambda}
$$

Thus, for example, a disk with radius $R=2 \lambda$ the geometrical acoustics is valid up to a distance from the disk $d_{\text {geo }} \leq 4 R$.

After a short introduction to thin lens theory in paragraph 5.1, geometrical acoustics (or geometrical optics) of mainly spherically concave surfaces is discussed in paragraph 5.2.

In paragraph 5.3 the geometrical methods used in computer-based calculations are discussed.

\section{$\underline{5.1 \text { Thin lens theory }}$}

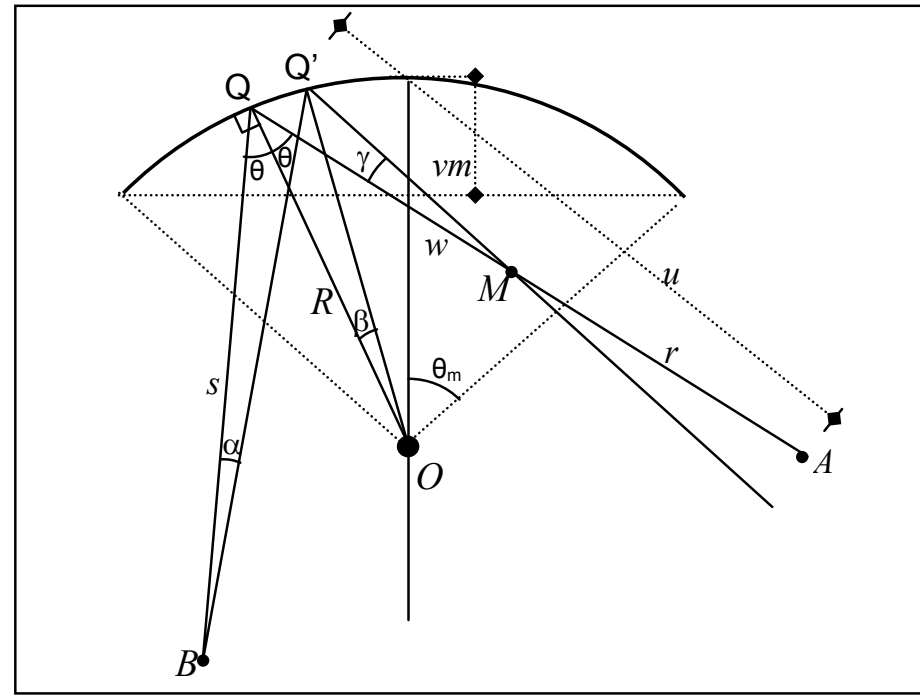

Figure 5.2. Geometry showing the concave surface with source $B$ and position of the focusing point $M$

Figure 5.2 shows the geometrical situation with a hard, reflecting, spherically concave surface characterized by the radius $R$, a point source $B$, a resulting focusing point $M$, and a receiver position $A$. For this situation the formula can be derived:

$$
\frac{1}{w}+\frac{1}{s}=\frac{2}{R \cos \theta}
$$

This is known (usually for normal incidence with $\cos \theta=1$ ) as the thin lens formula.

In optics this is usually formulated using the focal distance $f$ with $R=2 f$. This distance describes the position of the focusing point in the case of a source at the axis, at an infinite source distance $s$. 
Derivation:

Figure 5.2 shows the distances $s=B Q, w=M Q, r=M A$ and, from $B, O$ and $M$, the angles between the lines to $Q$ and $Q$ ' are named $\alpha, \beta$ and $\gamma$.

The geometry shows that:

$$
Q Q^{\prime}=\frac{s \cdot \sin \alpha}{\cos \theta}=\frac{w \cdot \sin \gamma}{\cos \theta}=R \cdot \sin \beta
$$

for small angles of $\alpha, \beta$ and $\gamma$ :

$$
s \alpha=w \gamma=R \beta \cdot \cos \theta
$$

this will give:

$$
\begin{aligned}
& w=s \cdot \frac{\alpha}{\gamma} \quad \text { and } \\
& \beta=\frac{s \cdot \alpha}{R \cdot \cos \theta}
\end{aligned}
$$

In the triangle $O Q Q^{\prime}$ the angles $O Q Q^{\prime}$ and $O Q Q^{\prime} Q$ will be named: $\varphi$.

So for triangle $O Q Q: \quad \beta+2 \varphi=\pi \Rightarrow \varphi=\frac{1}{2}(\pi-\beta)$,

for triangle SQQ: $\quad \alpha+(\varphi+\theta)+\left(\varphi-\theta^{\prime}\right)=\pi \Rightarrow \theta-\theta^{\prime}=\pi-\alpha-2 \varphi=\beta-\alpha$

and for triangle $M Q Q^{\prime}: \quad \gamma+(\varphi-\theta)+\left(\varphi+\theta^{\prime}\right)=\pi \Rightarrow \theta-\theta^{\prime}=\gamma-\beta=\beta-\alpha$

It follows that:

$$
\gamma=2 \beta-\alpha
$$

this will result in:

$$
w=\frac{1}{\frac{2}{R \cos \theta}-\frac{1}{s}}, \text { or: } \frac{1}{w}+\frac{1}{s}=\frac{2}{R \cos \theta}
$$

From classical optics the following cases can be considered:

$s<f \cos \theta$ : the geometrical sound field will be diverging and $w$ will be negative.

$s=f \cos \theta:$

the geometrical sound field will be neither converging nor diverging (if the mirror would be parabolic, the geometrical sound field would be parallel).

$f \cos \theta<s<R \cos \theta$ : the sound field will be converging to a focusing point $>R$. $s \approx R \cos \theta:$ the sound field will converge in a focussing point, that is the mirror point of source position $B$, mirrored point-symmetrically around the center point $O$. (if $B$ is in the centre point then $M$ will be in the centre point).

$s>R \cos \theta: \quad$ the sound field will converge in a focusing point between $f$ and $R$.

For geometric acoustic modeling of spherically curved surfaces, the focusing point position $M$ can be seen as a mirror source analogous to the Image Source Method (ISM), even in the situation that it is inside the room. 


\section{$\underline{5.2 \text { Geometrical sound field }}$}

\section{$\underline{\text { 5.2.1 Spherical surfaces }}$}

The sound pressure at distance $s$ from a monopole will be described by:

$$
p(s)=\hat{p} \frac{e^{-j k s}}{|s|}
$$

where $\hat{p}[\mathrm{~N} / \mathrm{m}]$ is the amplitude descriptor that represents the pressure at $1 \mathrm{~m}$ from the source.

Note: $|p(1)|=\hat{p} / 1 \mathrm{~Pa}$

It can be shown (see [WulfftenPalthe76], [Kuttruff73]) that for a small sound source (compared to wavelength):

$$
\hat{p}=\frac{j \omega \rho}{4 \pi} U
$$

where $U$ is the volume velocity $\left[\mathrm{m}^{3} / \mathrm{s}\right]$ of the monopole source.

The geometrical reflection field can be described using a mirror source at the position of the focusing point. The pressure caused by this mirror source will depend on the distance between $r$ and the focusing point (see figure 5.2):

$$
p(r)=X \frac{e^{-j k r}}{|r|}
$$

The amplitude descriptor $X$ can be determined by setting the pressure from the (real) source and the mirror source at the focusing point equal at the surface of the concave reflector:

$$
\hat{p} \frac{e^{-j k s}}{|s|}=X \frac{e^{-j k w}}{|w|}
$$

Since at the mirror, the phase of the pressure of the reflection will equal to the phase of the incident pressure, the amplitude of the (mirror) source at the focusing point will be:

$$
X=\hat{p}\left|\frac{w}{s}\right|
$$

The reflected sound field can now be described by:

$$
p(r)=\hat{p} \cdot\left|\frac{w}{s}\right| \cdot \frac{e^{-j k(s+w+r)}}{|r|}=\hat{p} \cdot\left|\frac{w}{s}\right| \cdot \frac{e^{-j k(s+u)}}{|u-w|}
$$

where $u$ is the distance of the receiver to the surface $(u=w+r)$. This is also valid for small $s(s<f \cos \theta)$ with negative $w$. In that case, $r=u$-w will represent the distance from position $M$ on the other side of the reflector to the receiver. 
The increase or decrease of the sound pressure level compared to the sound level at $1 \mathrm{~m}$ from the source will be:

$$
\Delta L_{1 m}=20 \lg \left(\left|\frac{w}{s}\right| \cdot \frac{1}{|u-w|}\right)=20 \lg \left(\left|\frac{R \cos \theta}{2 s-R \cos \theta}\right| \frac{1}{|u-w|}\right)=-20 \lg \left(\left|\frac{2 u s}{R \cos \theta}-(u+s)\right|\right)
$$

In this expression the first term results from the convergence of the sound field, caused by the concave reflector, and the second term is the geometrical distance. At distance $u$ from a flat surface the amplitude of the reflection will be:

$$
|p(u)|=\frac{\hat{p}}{s+u}
$$

From (5.10) and (5.12) the amplification $q$ of the pressure from the concave surface (relative to the flat surface) will be:

$$
q=(s+u) \frac{w}{s} \cdot \frac{1}{|u-w|} \Rightarrow \frac{1}{q}=\left|\frac{1}{\left(\frac{1}{u}+\frac{1}{s}\right)} \frac{2}{R \cos \theta}-1\right|
$$

The sound pressure level increase compared to a flat reflector will be:

$$
\Delta L_{c}=-20 \lg \left(\left|\frac{1}{\left(\frac{1}{u}+\frac{1}{s}\right)} \frac{2}{R \cos \theta}-1\right|\right)
$$

At a receiver position the difference between the reflected sound and the direct sound $\Delta L$ will be composed of the decrease with distance of the reflection $\Delta L_{r}$ and the amplification due to the curvature $\Delta L_{c}$ :

$$
\Delta L=\Delta L_{r}+\Delta L_{c}
$$

\section{$\underline{5.2 .2 \text { cylindrical surfaces }}$}

Formula (5.14) can be interpreted as the SPL increase of a double curved surface with radius of curvature $R$ in both directions.

If the radius of curvature $R$ is different in say $x$ - and $y$-direction ( $z$-direction being the axis from the centre through the middle of the curved segment), the sound pressure level increase can be separated in the $x$ - and $y$-directions:

$$
\Delta L_{c}=-10 \lg \left(\left|\frac{1}{\left(\frac{1}{u}+\frac{1}{s}\right)} \frac{2}{R_{x} \cos \theta_{x}}-1\right|\right)-10 \lg \left(\left|\frac{1}{\left(\frac{1}{u}+\frac{1}{s}\right)} \frac{2}{R_{y} \cos \theta_{y}}-1\right|\right)
$$

For cylindrically curved surfaces $\left(R_{y} \rightarrow \infty\right)$ this reduces to:

$$
\Delta L_{c}=-10 \lg \left(\left|\frac{1}{\left(\frac{1}{u}+\frac{1}{s}\right)} \frac{2}{R_{x} \cos \theta_{x}}-1\right|\right)
$$


This expression corresponds to the expressions given by [Kuttruff73] and [Rindel85], (see also figure 5.3). Convex surfaces can be calculated by using negative $R$.

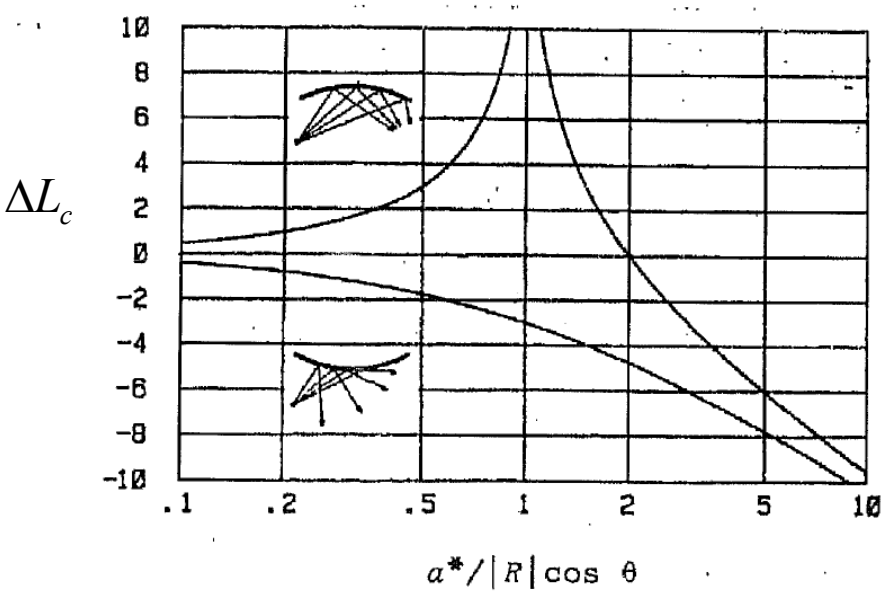

Figure 5.3. Attenuation due to cylindrically curved surfaces, relative to the reflection from a flat panel, with $a^{*}=\frac{2 s u}{s+u}=\frac{2}{\left(\frac{1}{u}+\frac{1}{s}\right)}$, from [Rindel85]

For example, for a cylinder with the source in the centre, we can obtain the pressure of the reflected signal at distance $u$ from the reflecting surface (the travelled path of the sound is $R+u$ ):

$$
L_{p}=L_{p 1 m}-20 \lg (R+u)+\Delta L_{c}
$$

Using $s=R, \cos \theta=1$ we can obtain from (5.17):

$$
\begin{aligned}
\Delta L_{c} & =-10 \lg \left(\left|\frac{1}{\left(\frac{1}{u}+\frac{1}{R}\right)} \frac{2}{R}-1\right|\right) \\
& =-10 \lg \left(\left|\frac{u-R}{R+u}\right|\right)
\end{aligned}
$$

The pressure at the receiver will be, from (5.18):

$$
L_{p}=L_{p 1 m}-10 \lg \left(\left|(R+u)^{2} \frac{u-R}{R+u}\right|\right)=L_{p 1 m}-10 \lg \left(\left|R^{2}-u^{2}\right|\right)
$$

It is noted that for $u=w$ (at the mirror point $M$ ) the calculated sound pressure according to (5.10) will be infinite. This is the case for :

$$
\frac{1}{\left(\frac{1}{u}+\frac{1}{s}\right)}=\frac{1}{2} R \cos \theta
$$

This is the basic limitation of the geometric model. A high $\Delta L_{c}$ indicates that the position is close to the focus and therefore a wave-based approximation should be used. 


\section{$\underline{5.2 .3 ~ E l l i p t i c a l ~ s u r f a c e s ~}$}

For an elliptical surface (elliptical cylinder) the radius of curvature depends on the reflection point on the ellipse.

For an ellipse described by:

$$
\frac{x^{2}}{a^{2}}+\frac{y^{2}}{b^{2}}=1
$$

with $a$ and $b$ the semi-major and semi-minor axis of the ellipse $(a>b)$.

The radius of curvature of an ellipse, as a function of $x$, is given by:

$$
R=\frac{1}{a b}\left(a^{2}-\left(1-\frac{b^{2}}{a^{2}}\right) x^{2}\right)^{3 / 2}
$$

The maximum and minimum radius will be $R=a^{2} / b$ for $x=0$ and $R=b^{2} / a$ for $x=a$.

Based on Snel's law, the reflection point on the ellipse can be determined. However the solution of this equation requires a numerical approach.

Since an exact determination of the reflection point is generally not necessary, having only an indication of the reflection point will be sufficient to estimate the local radius of curvature; the reflection point $x$ on the ellipse can be obtained from drawing the ellipse, tangents and reflections.

The radius of curvature from (5.22) can then be applied to (5.17) to calculate the $\Delta L_{c}$.

For prolate ellipsoid shapes that have a circular shape in cross section and an elliptical shape in longitudinal section (see e.g., par. 14.5), the geometrical amplification can be calculated in the same way, using (5.16), 


\section{$\underline{5.2 .4 \text { Other shapes }}$}

The methods described in the preceding paragraphs are applicable for spherical, cylindrical or elliptical curved surfaces. For other curvatures a more general expression can be given for the geometrical amplification (e.g., [Makrinenko86]):

$$
\Delta L_{c}=10 \lg \left(S_{u} / S_{r}\right)
$$

where $S_{u}$ is the area of the undisturbed spherical wavefront, for the relevant angle of incidence and $S_{r}$ is the area of the reflected geometrical wavefront. By "manually" constructing the wavefront as illustrated in figure 5.1, the area of this reflected wavefront can be determined.

Geometrical acoustics can be applied outside the focusing point or when the focusing point is not formed. Another presumption, which was already mentioned in the introduction, is that the wave length is considerably smaller than the dimensions of the reflecting surface. 


\section{$\underline{5.3 \text { Geometrical computer models }}$}

Computer models are used as a prediction tool for practical room acoustical purposes. The common prediction models are based on geometrical acoustics. Methods used are Image Source Methods (ISM), Ray Tracing (RT) and Beam Tracing (BT). In this paragraph the possibilities will be investigated for using computer-based geometrical methods for the calculation of reflections from concave surfaces in and around the focusing point. Further, there will be an evaluation of the differences between the calculation results and reality.

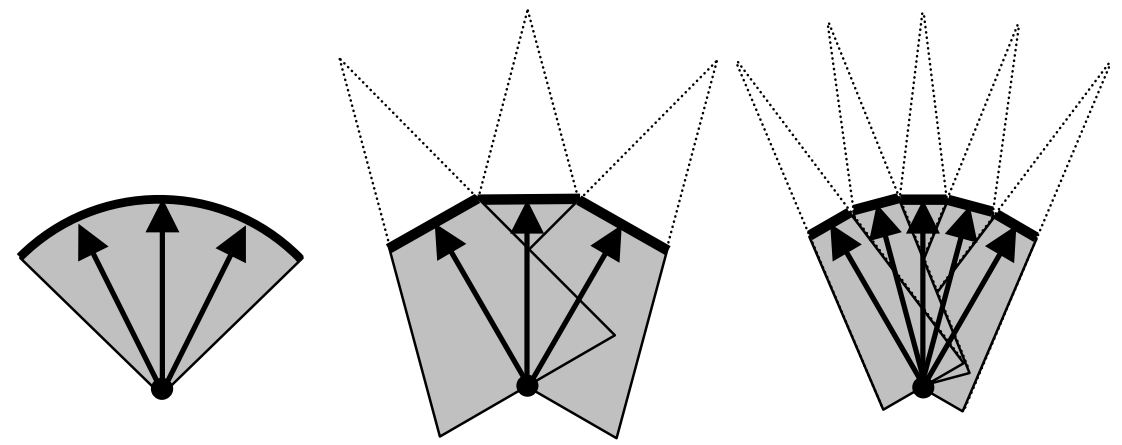

Figure 5.4. Illustration of the geometrical reflection by a continuous curvature (left) and segmented curvatures (middle and right)

In the practice of room acoustic modeling, curved (sphere) elements are not modeled as curved elements but are replaced by small plane surfaces, segmenting the curved element (see figure 5.4). Depending on the shape of the curved segment, they are modeled as rectangular, trapezium or triangular planes.

This segmenting will influence the calculated pressure in the focusing point.

In this paragraph the influence of segmenting the curved surface will be discussed. This influence will depend on the method used. First, the image source method will be discussed.

For all geometrical methods wave phenomena are basically not included. It is assumed that the (curved) reflector is large compared to the wavelength, thus this approach is valid for relatively high frequencies.

In geometrical models the signals can be added in two ways:

1. energy based

The energy (proportional to $p^{2}$ ) of reflections from different surfaces or surface elements is added. The contributions are considered to be non-coherent. Phase is not considered and recording interference is not possible.

2. pressure based

The pressure due to reflections from different surfaces or surfaces is added, also the phase of the signal is considered. The contributions are from "coherent" (mirror) sources. In this concept interference is possible.

The geometrical methods described in paragraph 5.2 are basically energy-based. Most of the geometrical computer-based methods are energy-based, some of them however can also be pressure based, as will be indicated. 


\subsubsection{Image Source Method}

\section{ISM with plane surfaces and mirror sources outside the model}

By "mirroring" a source relative to a surface, a mirror source can be constructed. Assuming full (geometrical) reflection by the surface, the reflected sound field will be identical to the sound field by the mirror source, without the surface present. Although a "mirror" position (as the origin of a spherical wave) can also be constructed for some situations with curved surfaces (par. 5.2), the usual (computerbased) application is to construct mirror sources by mirroring in flat surfaces. Curved surfaces will be segmented into plane segments.

The sound pressure in the centre of a hemisphere, estimated using the Image Source Method (ISM), will be compared to the theoretical sound pressure. The source is also assumed to be in the centre of the hemisphere.

Assuming the plane surfaces have characteristic dimension $b$, the area of each element will be $b^{2}$. Applying ISM for a hemisphere the number of mirrors will be:

$$
N=2 \pi R^{2} / b^{2} .
$$

Conventionally in ISM the energy of the visible image sources is added.

In that case, the pressure at distance $r$ from a (mirror) source can be written as:

$$
p(r)_{r m s}^{2}=\frac{1}{2} \hat{p}^{2} / r^{2} .
$$

With the source in the centre of a hemisphere, the pressure in the centre caused by all mirror sources at distance $r=2 R$ will be:

$$
p(0)_{r m s}^{2}=\frac{1}{2} N \hat{p}^{2} /(2 R)^{2} .
$$

In chapter 7 it will be derived that the pressure in the focusing point of a hemisphere is: $|p(0)|=\hat{p} k$ and $p(0)_{r m s}^{2}=\frac{1}{2} \hat{p}^{2} k^{2}$. This value can be obtained by segmenting in plane surfaces, when the optimal number of plane surfaces $N$ is applied (using $k=2 \pi(\lambda)$ :

$$
N=4 R^{2} k^{2}=(4 \pi R / \lambda)^{2} .
$$

That means that the number of surfaces required will be frequency dependent. For real situations in room acoustics, for example a frequency of $500 \mathrm{~Hz}$ and a radius $R=10 \mathrm{~m}: N>34000$. This is not practically feasible. The large value for $N$ is due to the summation of energy, which is basically not correct for coherent sources.

Although conventional applications of ISM are based on the addition of energy, this is not necessary since the phase and traveled distance of the signal from all mirror sources are known (see e.g.[Allen79]). If pressure-based addition is used, the number of surfaces can be reduced. Assuming a point source in the centre of the sphere: $p(r)=\hat{p} e^{-i k r} / r$, the contribution of each image source in the centre will be $p(r)=\hat{p} e^{-i k 2 R} / 2 R$. When adding all image sources (with the same phase) the total pressure in the centre will be:

$$
|p(0)|=N \hat{p} / 2 R \text {. }
$$


When comparing it to the correct pressure in the focusing point of a hemisphere $(|p(0)|=\hat{p} k)$, a correct prediction in the focusing point could be obtained by applying an optimized number of image source $N$ :

$$
N=2 k R=4 \pi R / \lambda \text {. }
$$

For a correct prediction in the focusing point, exactly this frequency dependent number of surfaces would be required, not less but also not more. For the calculation example given above $N=370$ is needed at $500 \mathrm{~Hz}$. This is much less than in the approach using energy summation and might even be practically possible.

The width of the surfaces will be:

$$
b=\sqrt{2 \pi R^{2} / N}=\sqrt{\frac{1}{2} \lambda R}
$$

In this example $b=1,3 \mathrm{~m}$. This agrees with the required width of plane segments to model a cylinder found by [Kuttruff93]. The illuminated width in the centre by the mirror sources will be $2 b$, in this case $2,6 \mathrm{~m}$. In paragraph 7.4 .4 it is shown that in the case of a hemisphere the actual width at the focusing point is approximately $\frac{1}{2} \lambda$. Thus, when $R>2 \lambda$, which is usually the case (except in small rooms at low frequencies), the focusing area calculated with mirror images is considerably larger than $\frac{1}{2} \lambda$. So by segmenting the curved surface and applying ISM it is not possible to correctly predict both focusing strength and width of the focusing area.

The next question is whether the distribution outside the focusing area can be calculated correctly.

When moving the observation point away from the centre, the observation point will be out of the visibility zone of a number of image sources. The number of visible image sources for a hemisphere will be calculated while moving the receiver along the $z$-axis.

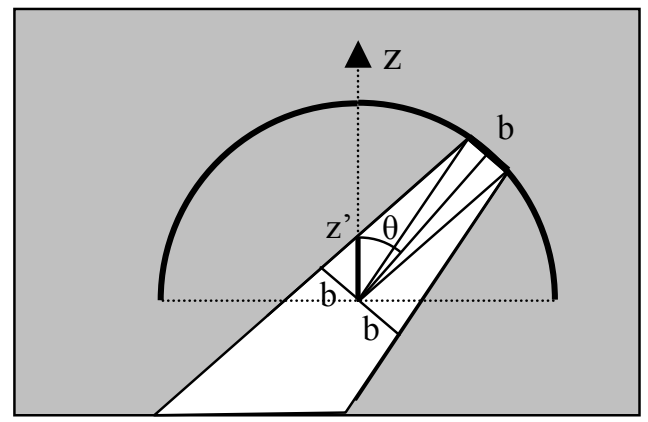

Figure 5.5. Illustration of the illuminated part of the z-axis by a plane segment.

The illuminated length along the z-axis by a single plane surface with dimensions $b \cdot b$ can be approximated (for small $b$ ) by $z^{\prime}=b / \sin \theta$ (see figure 5.5 ). For position $z^{\prime}: \theta_{v}=\arcsin \left(b / z^{\prime}\right)$, with $z^{\prime} \geq b$. On the $z$-axis, all mirror sources will be visible for $z^{\prime}$ for which $0<\theta<\theta_{v}$. In the horizontal plane the observation point at $x=y=0$ will be visible for all mirror images (provided that $0<\theta<\theta_{v}$ ). For position $z^{\prime}$ the total number of mirror sources visible $n\left(z^{\prime}\right)$ will be the area of the hemisphere where the sources are visible, divided by the area per segment:

$$
n(z)=\frac{R^{2}}{b^{2}} \int_{0}^{2 \pi \theta_{0}} \int_{0} \sin \theta d \theta d \varphi=\frac{2 \pi R^{2}}{b^{2}}\left[1-\sqrt{1-\frac{b^{2}}{z^{2}}}\right]
$$


This results in a sound pressure:

$$
p(z)_{r m s}^{2}=\frac{n(z) \hat{p}^{2}}{8 R^{2}}=\hat{p}^{2} \frac{\pi}{4 b^{2}}\left[1-\sqrt{1-\frac{b^{2}}{z^{2}}}\right]
$$

For $z \geq b$ the rms pressure can be estimated by the first two terms of the Taylor series:

$$
p(z)_{r m s}^{2} \approx \frac{\pi \hat{p}^{2}}{8 z^{2}}
$$

When $z$ varies from $b$ to $z>>b$, the pressure $p(z)_{r m s}^{2}$ will vary from $\frac{\pi}{4} \hat{p}^{2} / z^{2}$ to $\frac{\pi}{8} \hat{p}^{2} / z^{2}$, which comes close to the geometrical decrease with distance:

$$
p(z)_{r m s}^{2}=\frac{1}{2} \hat{p}^{2} / z^{2}
$$

In reality, the calculated pressure will vary stepwise. Figure 5.6 shows the calculated pressure along the $z$-axis of a hemisphere $(R=10 \mathrm{~m})$ for $N=255$ for the situation that the surfaces have equal area $b^{2}$. For $z>b$ (in this case $b \approx 1,5 \mathrm{~m}$ ) this corresponds quite well to the geometrical decrease with distance.

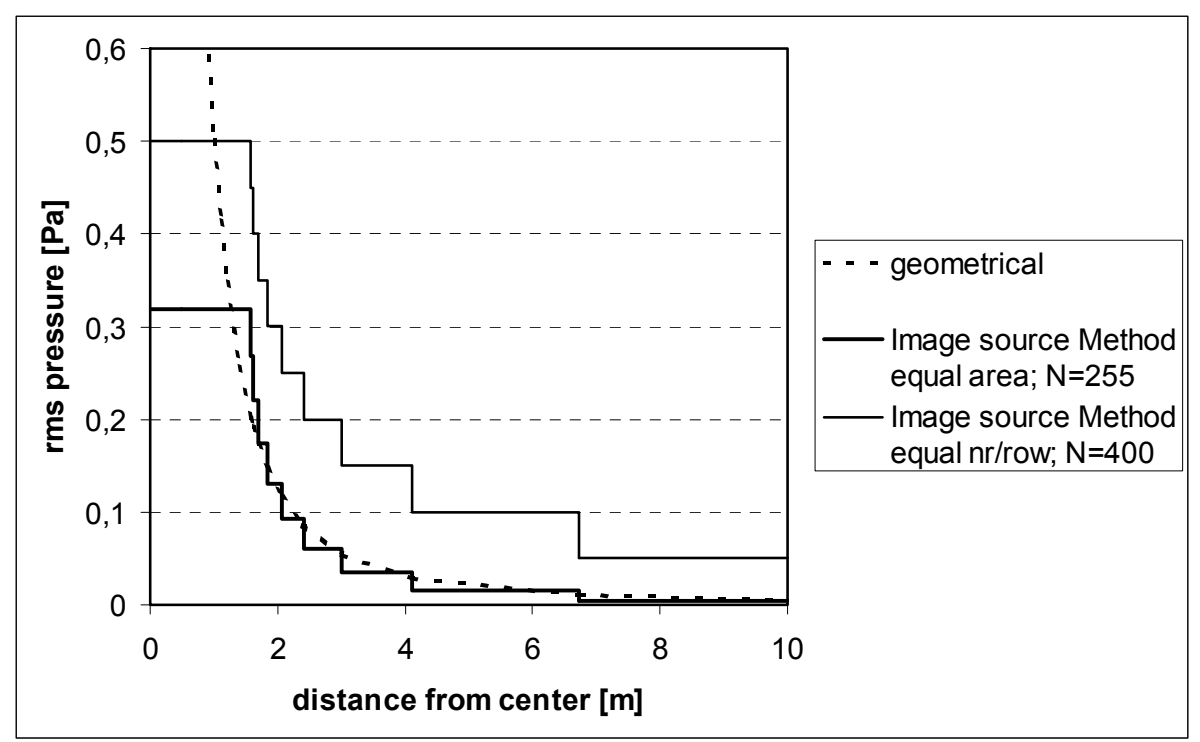

Figure 5.6. Rms Sound Pressure along the z-axis of a hemisphere with the sound source ( $\hat{p}=1 \mathrm{~N} / \mathrm{m}$ ) in the centre, geometrical propagation (5.32) and ISM with segmented modeling of the hemisphere, with surfaces with equal area and with surfaces with different areas (constant number of surfaces in each row).

A requirement of room acoustical computer models is that these models are closed: without openings that result in loss of rays. For a sphere this can not be done with equal surfaces $b \cdot b$. Figure 5.6 also shows the calculation result when the number of surfaces is kept constant in each row, to be able to connect the corners. Moving upward in the cupola the area of the plane surfaces decreases. However, with ISM, the contribution to the pressure on the $x$-axis depends on the number of surfaces, not on the area of each surface. The total number of surfaces is 400 . Quite different results can be seen, which do not correspond to the geometrical decrease.

It can be concluded that even out of the focusing point the use of segmentation in combination with ISM can produce quite erroneous results when modeling a spherical reflector. 


\section{ISM with mirror source inside the model}

Improving the prediction of the reflection by concave surfaces, especially in the focusing area, is only possible by considering the curved surfaces as such, instead of segmenting the model.

The introduction to this chapter mentions that for spherically concave surfaces the focusing point position $M$ can be seen as a mirror source, with a source strength as described in paragraph 5.2.

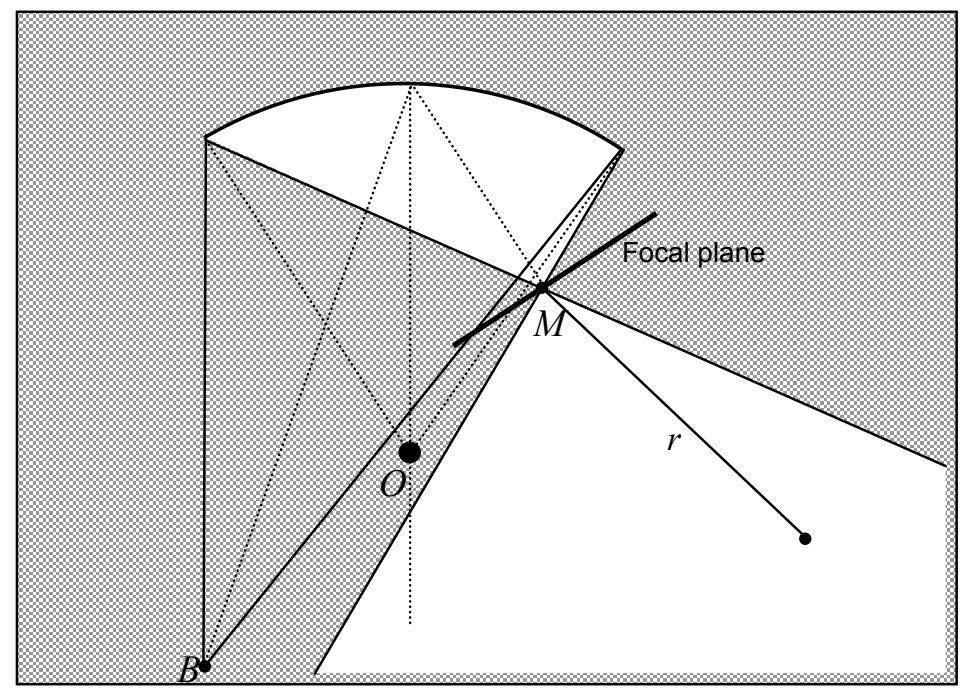

Figure 5.7. Geometry showing the illuminated reflection area in white and the receiver at distance $r$ from the focusing point. The area outside the illuminated area is a shadow zone for the reflection.

With this mirror source, the reflected sound field can be described outside the focusing point. Outside this focusing point the amplitude does not depend on the size of the mirror; the reception point is either visible or not.

The visibility check is basically similar to ordinary mirror sources. The mirror source is visible (or "a reflection has to be calculated") when a line from the receiver point through the mirror source hits the concave surface. The area where this is the case, the illuminated area, is shown in figure 5.7.

One difference between mirror sources for concave surfaces and mirror sources for flat surfaces is that for flat surfaces the surface is between the reception point and the mirror source. With concave surfaces the mirror source can be between the reception point and the reflecting surface.

It can be concluded that it is geometrically possible to use mirror imaging with concave spherical surfaces; the mirror image is then located inside the reflection space. The amplitude and time delay from the mirror source and the visibility check have to be adapted.

For other curved surfaces, such as cylindrical shapes this is not possible, since there is not a single focusing point. 


\subsubsection{Ray Tracing}

Ray tracing is used in many fields such as optics, seismics and acoustics.

The propagating wave is modeled with a ray in the propagating direction.

The source is emitting the rays mostly, but not necessarily, with a uniform distribution. The addition of rays is energy-based.

If we assume the squared sound pressure at distance $r$ of a monopole to be:

$$
p_{r m s}(\omega)^{2}=\frac{1}{2} \frac{\hat{p}^{2}}{r^{2}}
$$

then the sound power of this source will be (intensity $I$ and surface $S$ calculated at distance $r$ ):

$$
P=I \cdot S=\frac{\hat{p}^{2}}{2 \rho c \cdot r^{2}} \cdot 4 \pi r^{2}=\frac{2 \pi}{\rho c} \hat{p}^{2}
$$

Assuming a uniform distribution of $N$ rays emitted by the source, the power represented by each ray $i$ will be:

$$
P_{i}=\frac{2 \pi}{\rho c} \frac{\hat{p}^{2}}{N}
$$

Note: In the general case of a non-uniform source (with directivity factor $Q_{i}$ for ray $i$ ) the power of ray $i$, after $J$ reflections from surfaces with absorption factor $\alpha_{j}$ and having traveled over a distance $L$ with air absorption $m$, will be [Xiangyang03]:

$$
P_{i}=\frac{2 \pi}{\rho c} \frac{\hat{p}^{2} Q_{i}}{N} e^{-m L} \sum_{j=1}^{J}\left(1-\alpha_{j}\right)
$$

The rays are detected using a spherical receiver volume. The energy in that receiver volume depends on the travel time of the ray through the volume:

$$
E_{i}=P_{i} \cdot \Delta t
$$

with $\Delta t=\frac{l_{i}}{c}$, where $l_{i}=$ the path length of ray $i$ through the receiver volume.

The average intensity (averaged over the volume of the receiver) of the sound wave of ray $i$ inside the receiver is [Xiangyang03]:

$$
I_{i}=\frac{E_{i} \cdot c}{V}
$$

where $V=$ volume of the receiver. This will result in:

$$
I_{i}=\frac{P_{i} \cdot \Delta t \cdot c}{V}=\frac{P_{i} \cdot l_{i}}{V}
$$

In a situation where the model of a curved surface is segmented and the source is in the centre, there will be a spread of energy around the focusing point at the centre (see also figure 5.4). If this spread is limited to $\frac{1}{2} \lambda$ width, as it is the case for a hemisphere (see par. 7.4.4), the dimensions of the segments should be $\frac{1}{4} \lambda$. Contrary to the mirror source method, the pressure in the focusing point will not increase when the number of segments increases, since the number of rays that hit 
the focusing area depends on the number of rays emitted from the source and the total opening angle of the concave surface.

Now an exact model is formulated in the sense that all rays reflect in the correct specular direction, depending on the orientation of the small surface element at the impact position of the ray. This model can either be a parameterized model or a sufficiently segmented model.

Note: [Mommertz95] attempts to improve the ray tracing technique by using the exact form instead of a segmented form. His conclusion is that a high number of rays is required as well as a small receiver volume to detect focusing effects. It is noted that the real sound field can only be predicted by wave field methods.

Next the case will be considered of a source in or around the centre of a full sphere and a receiver in the exact focusing point with all reflected rays passing this focusing point. There will be full reflection (i.e. no absorption). Assuming the centre of the receiver volume is in this focusing point, the path length $l$ will be equal to the diameter of the receiver volume.

The total pressure in the receiver volume (with diameter $D$ ) results from energy summation:

$$
p_{r m s}^{2}=\rho c \sum_{i=1}^{N} I_{i}=\rho c \sum_{i=1}^{N} \frac{P_{i} \cdot l_{i}}{V}=\rho c \sum_{i=1}^{N} \frac{P_{i}}{\frac{1}{6} \pi D^{2}}=12 \frac{\hat{p}^{2}}{D^{2}}
$$

This formulation will also be valid for sources outside the centre of the sphere, as long as it can be assumed that the reflected rays in the focusing point pass through the centre of the receiver volume. This expression is independent of the number of rays (as it should be) but also that it is dependent on the volume of the receiver. The energy is equally distributed over the receiver volume. A larger receiver volume will not be compensated by more rays (as it will be in a statistical sound field) since all rays pass through the centre.

In paragraph 7.4.4, it will be illustrated that in fact the energy will be distributed over an area depending on the wavelength. When we assume the diameter of the receiver volume $D=\frac{1}{2} \lambda$, the total pressure in the receiver volume at the focusing point, with the source in the centre, will be:

$$
p_{r m s}^{2}=12 \frac{\hat{p}^{2}}{D^{2}}=48 \frac{\hat{p}^{2}}{\lambda^{2}}
$$

while the correct solution for the pressure in the focusing point of a full sphere (see par. 7.2, formula (7.3c) with $\theta_{m}=\pi$ ) is:

$$
p_{r m s}^{2}=2 \hat{p}^{2} k^{2}=8 \pi^{2} \frac{\hat{p}^{2}}{\lambda^{2}}
$$

For this case it seems fairly possible, presuming a correct reflection direction of rays and the optimum (frequency dependent) selection of the receiver volume, to approximate the correct solution with the ray tracing procedure rather accurately. However, when we consider a hemisphere instead of a full sphere, only half of the rays will return to the focusing point. The total pressure using ray tracing and assuming a receiver size $D=\frac{1}{2} \lambda$ will be: 


$$
p_{r m s}^{2}=\rho c \sum_{i=1}^{\frac{1}{2} N} \frac{P_{i}}{\frac{1}{6} \pi D^{2}}=6 \frac{\hat{p}^{2}}{D^{2}}=24 \frac{\hat{p}^{2}}{\lambda^{2}}
$$

while the correct solution for the pressure in the focusing point of a hemisphere, with the source in the centre, will be (see par 7.2):

$$
p_{r m s}^{2}=\frac{1}{2} \hat{p}^{2} k^{2}=2 \pi^{2} \frac{\hat{p}^{2}}{\lambda^{2}}
$$

Now the ratio between the ray solution and the correct solution is different from the full sphere. This results from the energy summation. Energy summation is only correct in case of random phase. In case of sound focusing however, the sound waves arrive at equal phase, so basically pressure summation should be applied. Tricking an energy based summation for this purpose is only possible if you know the outcome beforehand.

It is noted that in commercially available ray tracing programs the size of the receiver can not be chosen.

Furthermore it is noted that only the pressure in the focusing point is calculated. For this particular case (all rays passing the receiver at an exact focusing point) there is no spread of energy calculated in the focusing plane. Due to the energy approach, the interference pattern is not calculated. Therefore, obtaining the right value by using ray tracing would be purely coincidental.

This is due to the fact that in the energy approach it is not possible to introduce the phase into the calculation. Thus, when the phase should be incorporated, beam tracing should be used. 


\subsubsection{Beam Tracing}

The main difference between Ray Tracing and Beam Tracing is the way that the decrease with distance is handled. In Ray Tracing the decrease of sound pressure of an expanding sound field with the distance from the source is implicitly in the calculation method since the distance between the rays becomes larger and in a statistical approach the probability of hitting a (fixed size) volume receiver decreases. Beam Tracing is deterministic in the sense that the whole radiating area of the source is covered. The decrease with distance is calculated from:

$$
p_{r m s}^{2} \cong \frac{\Delta \Omega}{S(r)}
$$

where $\Delta \Omega$ is the spatial opening angle of the beam and $S(r)$ is the cross-sectional area of the beam at distance $r$ from the source.

When applying this method to curved surfaces, the beam will converge and due to the smaller $S(r)$ the geometrically correct increase of sound pressure will be found. For example it is applied for the reflection of a spherical dome by [Moreno81]. In the focusing point however, $S(r)=0$ which leads to an (incorrect) infinite sound pressure. Therefore, this method is not capable of calculating the sound pressure in the focusing point.

Outside the focusing point however, this method can be applied and is expected to give basically the same results as ray tracing.

As with ray tracing, Beam Tracing is used as an energy-based method. Contrary to Ray Tracing, Beam Tracing is deterministic in the sense that at each position the pressure and phase can be calculated from (5.43) and the distance traveled.

Calculation results for pressure-based calculations on curved surfaces are reported in [Mommertz96] and [Jean07].

Outside the focusing point, coherent Beam Tracing is capable of determining the interference pattern, see figure 5.8. In this respect it is much better than the other methods. The main disadvantage of Beam Tracing is that the maximum pressure in the focusing point cannot be determined. The pressure is obtained from the square root of (5.43), still resulting in a singularity for $S(r)=0$.

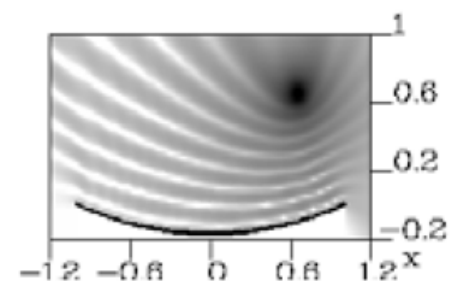

Figure 5.8. Calculation of coherent Beam Tracing for a concave surface, from [Jean07]. 


\section{$\underline{5.3 .4 \text { Overview of geometrical methods }}$}

Table 5.1 presents an overview of the possibilities and limitations of the geometrical methods presented above.

Table 5.1 Applicability of geometrical methods for reflections from concave surfaces

\begin{tabular}{|l|l|l|}
\hline Method & Applicability in focusing area & Applicability outside focusing area \\
\hline $\begin{array}{l}\text { ISM, segmented, } \\
\text { energy based }\end{array}$ & $\begin{array}{l}\text { Only correct for specific number } \\
\text { of segments (frequency } \\
\text { dependent) }\end{array}$ & $\begin{array}{l}\text { Rough approximation for } z>2 b \text { with } \\
\text { equal size segments, no interference }\end{array}$ \\
\hline $\begin{array}{l}\text { ISM, segmented, } \\
\text { pressure based }\end{array}$ & $\begin{array}{l}\text { Only correct for specific (but } \\
\text { lower) number of segments } \\
\text { (frequency dependent) }\end{array}$ & $\begin{array}{l}\text { Rough approximation for } z>2 b \text { with } \\
\text { equal size segments, with } \\
\text { interference }\end{array}$ \\
\hline $\begin{array}{l}\text { Image source } \\
\text { inside the space, } \\
\text { energy based }\end{array}$ & Not correct & $\begin{array}{l}\text { Good approximation, without } \\
\text { interference }\end{array}$ \\
\hline $\begin{array}{l}\text { Ray Tracing (RT), } \\
\text { energy based }\end{array}$ & $\begin{array}{l}\text { Rough approximation in the } \\
\text { case of receiver size } D=\frac{1}{2} \lambda ; \\
\text { too low for } D>\frac{1}{2} \lambda\end{array}$ & Rough approximation, no interference \\
\hline $\begin{array}{l}\text { Beam Tracing (BT) } \\
\text { energy based }\end{array}$ & Not correct & fair approximation, no interference \\
\hline $\begin{array}{l}\text { Beam Tracing (BT) } \\
\text { pressure based }\end{array}$ & Not correct & $\begin{array}{l}\text { Good approximation, with } \\
\text { interference }\end{array}$ \\
\hline
\end{tabular}

It can be concluded that none of the methods gives satisfactory results in the focusing point. In order to use ISM a very large (frequency dependent) number of segments is needed. The best (practical) results can be obtained with Ray Tracing if there are a sufficient (very large) number of rays and frequency dependent receiver size $D=\frac{1}{2} \lambda$.

The best results outside the focusing point, including the interference pattern, can be obtained with pressure-based Beam Tracing.

It can be concluded that better tools are needed than the geometrical computer models to approximate the sound pressure field, especially around the focusing point, due to reflections from concave surfaces.

Outside the focusing point, geometrical methods are sufficient to predict the average sound field. Interference might be incorporated by using pressure-based beam tracing.

The suitability of geometrical computer models (ISM and Ray Tracing) is also illustrated by a case study in paragraph 14.6. 


\section{Chapter6}

\section{Wave-based methods}

\subsection{The Huygens-Fresnel Principle}

The Huygens principle, which was developed by Christiaan Huygens in 1678, is based on the wave theory of light. The Huygens Principle states that every point on the primary wavefront can be thought of as an emitter of secondary wavelets. The secondary wavelets combine to produce a new wavefront in the direction of propagation.

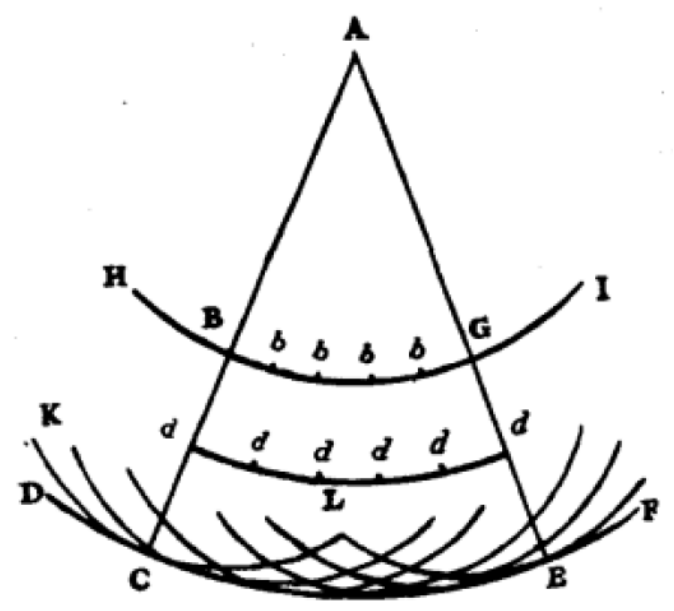

Figure 6.1. Huygens' depiction of the propagation of a spherical wavefront [Huygens1678]

But if every wavelet radiates in all directions uniformly there would also be a wavefront travelling back towards the source. Since no such wavefront is found the uniform radiation of the wavelets must be questioned (see par.6.3).

Augustin Fresnel extended the theory of Huygens, which is called the HuygensFresnel principle, by stating that the secondary wavelets mutually interfere, thus giving an explanation for the diffraction effects. At any given point the amplitude of the wave equals the superposition of the amplitudes of all the secondary wavelets at that point.

Fresnel also indicated the need for a law according to which the intensity of the wavelets varies.

It was Kirchhoff however, who put the Huygens-Fresnel principle on a sounder mathematical basis by formulating an expression for the obliquity or inclination factor. 


\subsection{The Kirchhoff integral}

An external force on a medium that has a static pressure and is at equilibrium will disturb this static pressure. These disturbances will be passed on in the medium. A wave will start travelling through the medium. Considering only the disturbance, the acoustic pressure is the difference between the in time varying total pressure and the static pressure. The acoustic pressure is time and location dependent.

The wave equation for a compressional wave in a homogeneous medium, without external forces, is:

$$
\nabla^{2} p(\bar{r}, t)-\frac{1}{c^{2}} \frac{\partial^{2} p(\bar{r}, t)}{\partial t^{2}}=0
$$

where: $p(\bar{r}, t)=$ the time dependent pressure at $\bar{r}=(x, y, z)$

$c=$ propagation velocity of compression waves

$$
\begin{aligned}
& \text { Using spherical coordinates with: } \\
& \qquad \begin{array}{l}
x=r \cdot \sin \theta \cdot \cos \phi \\
y=r \cdot \sin \theta \cdot \sin \phi \\
z=r \cdot \cos \theta
\end{array} \\
& \text { the wave equation will be: } \\
& \qquad \frac{\partial^{2} p}{\partial t^{2}}=c^{2} \cdot\left\{\frac{\partial^{2} p}{\partial r^{2}}+\frac{2}{r} \cdot \frac{\partial p}{\partial r}+\frac{1}{r^{2}} \cdot \frac{\partial^{2} p}{\partial \theta^{2}}+\frac{\cot \theta}{r^{2}} \cdot \frac{\partial p}{\partial \theta}+\frac{1}{r^{2} \sin ^{2} \theta} \cdot \frac{\partial^{2} p}{\partial \phi^{2}}\right\}
\end{aligned}
$$

For a wave originating in $(r, \theta, \phi)=(0,0,0)$ and independent of $\phi$ or $\theta$ this will reduce to:

$$
\frac{\partial^{2} p}{\partial t^{2}}=c^{2} \cdot\left\{\frac{\partial^{2} p}{\partial r^{2}}+\frac{2}{r} \cdot \frac{\partial p}{\partial r}\right\}
$$

One solution of this equation is:

$$
p(r, t)=\hat{p} \cdot \frac{e^{j(\omega t-k r)}}{r}=\hat{p} \cdot \frac{e^{-j k r}}{r} \cdot e^{j \omega t}
$$

In the frequency domain the wave equation can be written as the Helmholtz equation:

$$
\nabla^{2} P(\bar{r}, \omega)+k^{2} P(\bar{r}, \omega)=0
$$

where: $k=$

$$
\begin{array}{ll}
\omega= & \text { angular frequency of the signal } \\
P(\bar{r}, \omega)= & \text { Fourier transformation of the time signal } p(\bar{r}, t)
\end{array}
$$

This is a homogeneous equation, thus it excludes external forces. This applies for a volume $V$ with the sound source outside this volume.

Say volume $V$ has the boundary surface $S$ and point $A$ within volume $V$. A disturbance in a specific point at surface $S$ will result in a disturbance at point $A$. However, the transmission from a point at surface $S$ to point $A$ will be the same as the reverse: the transmission of a disturbance from $A$ to that point on surface $S$.

In the case of a disturbance by a source in point $A$, the wave field at distance $u$ from the source can be described by a function $G(u, \omega)$ that fulfills the following condition (unit excitation): 


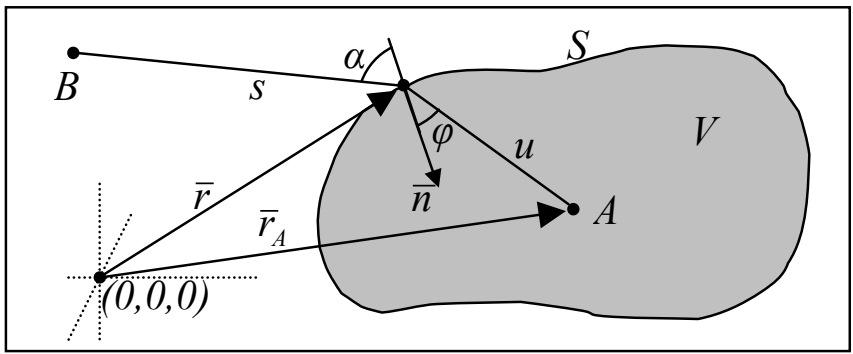

Figure 6.2. Geometry and notation used

$$
\nabla^{2} G(u, \omega)+k^{2} G(u, \omega)=-4 \pi \delta(u)
$$

where: $\delta(u)=$ the spatial delta pulse

A particular solution of this equation will be

$$
G(u, \omega)=\frac{e^{-j k u}}{u}
$$

For two functions $P$ and $G$, which are continuous for their first and second derivatives, Greens theorem (or Green's "second identity") can be applied:

$$
\left.\int_{V} P \nabla^{2} G-G \nabla^{2} P\right) d V=\int_{S}(P \nabla G-G \nabla P) \cdot \bar{n} d S
$$

where: $\bar{n}=$ normal vector on surface $S$, pointing inward.

If the functions $P$ and $G$ fulfill the homogeneous Helmholtz equation, the left part of (6.5) will be zero, so the surface integral with first order derivatives of $P$ and $G$ will remain:

$$
\int_{S}(P \nabla G-G \nabla P) \cdot \bar{n} d S=\int_{S}\left(P \frac{\partial G}{\partial n}-G \frac{\partial P}{\partial n}\right) d S=0
$$

However, function $G$ is not continuous at the source position $A$, so the preconditions of Greens theorem are not fulfilled. This problem can be solved by creating a small sphere with radius $\varepsilon$ around this source in $A$, as indicated in figure 6.3 (see e.g., [Born59]). The total surface $S$ of the integration will be: $S=S_{o}+S_{i}$, so:

$$
\int_{S_{o}}\left(P \frac{\partial G}{\partial n}-G \frac{\partial P}{\partial n}\right) d S+\int_{S_{i}}\left(P \frac{\partial G}{\partial n}-G \frac{\partial P}{\partial n}\right) d S=0
$$

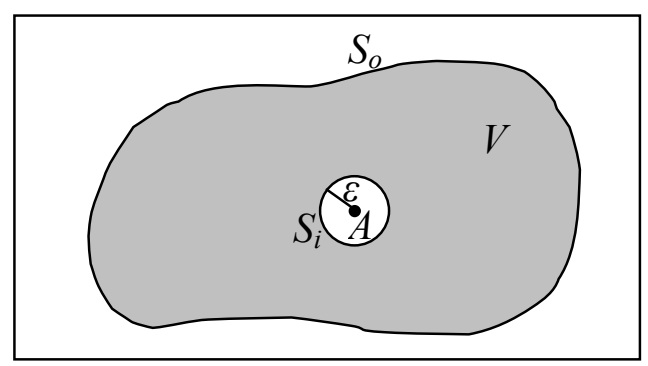

Figure 6.3. Creation of a small sphere around point $A$ for function $G$.

For $\varepsilon \rightarrow 0$ the second part of the integral over $S_{i}$ will be zero since $G$ will be constant and $\partial P / \partial n$ will cancel out over $S_{i}$. For the first part of the integral over $S_{i}$ (with the normal $n$ pointing inward, so opposed to $\varepsilon$ ): 


$$
\begin{aligned}
& \frac{\partial G}{\partial n}=-\frac{\partial G}{\partial \varepsilon}=-\frac{j k \varepsilon \cdot e^{j k \varepsilon}-e^{j k \varepsilon}}{\varepsilon^{2}}=-\left(j k-\frac{1}{\varepsilon}\right) \frac{e^{j k \varepsilon}}{\varepsilon} \approx-\frac{e^{j k \varepsilon}}{\varepsilon^{2}} \\
& \lim _{\varepsilon \rightarrow 0} \int_{S_{i}} P \frac{\partial G}{\partial n} d S=\lim _{\varepsilon \rightarrow 0}-p\left(\bar{r}_{A}\right) \int_{S_{i}} \frac{e^{j k \varepsilon}}{\varepsilon^{2}} d S=-4 \pi p\left(\bar{r}_{A}\right)
\end{aligned}
$$

With this result for the integral over $S_{i}$, the integral equation (6.7) will result in:

$$
p\left(\bar{r}_{A}\right)=\frac{1}{4 \pi} \int_{S_{o}}\left(P \frac{\partial G}{\partial n}-G \frac{\partial P}{\partial n}\right) d S
$$

Note: If point $A$ is at the (continuous) boundary, the source in $A$ will radiate over half a sphere only and the surface integral will be equal to $\frac{1}{2} p\left(\bar{r}_{A}\right)$. If point $A$ is outside $V$, the surface integral will be zero.

Equation (6.10) means that the pressure in every receiver point $A$ inside $V$ can be derived from the surface integral over functions $P$ and $G$ on that surface and their derivatives, where $P$ can be considered the pressure $p(\bar{r})$ on the surface from one or more sources outside $V$, and $G$ is the Green function describing the monopole radiation from internal point $A$ to the surface.

At the surface $S_{o}$ the derivative of $G$ in the direction of $\bar{n}$ will be:

$$
\frac{\partial G}{\partial n}=-\frac{-j k \cdot e^{-j k u} u-e^{-j k u}}{u^{2}} \cos \varphi=\left(j k+\frac{1}{u}\right) \cos \varphi \frac{e^{-j k u}}{u}
$$

where: $u=\left(\bar{r}-\bar{r}_{A}\right)=\sqrt{\left(x_{A}-x\right)^{2}+\left(y_{A}-y\right)^{2}+\left(z_{A}-z\right)^{2}}$ and

$\cos \varphi$ is the cosine of the angle between $\bar{n}$ and the line from $A$ to the point on surface $S_{o}$, in fact this is a dipole radiation pattern (figure 6.4)

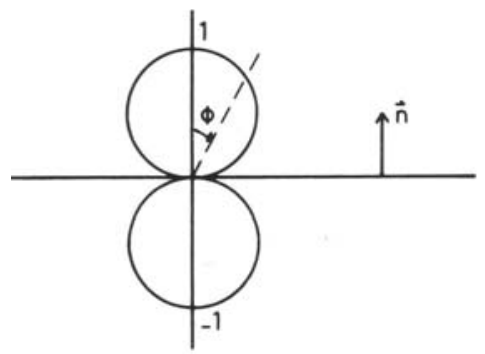

Figure 6.4. Directivity pattern of a dipole.

From Newton's second law it is known that:

$$
\frac{\delta p(\bar{r})}{\delta n}=-j \omega \rho \cdot v_{n}(\bar{r})
$$

where: $v_{n}(\bar{r})=$ velocity in the direction of $\bar{n}$, normal to the surface.

So the pressure in $A, p\left(\bar{r}_{A}\right)$, can be written:

$$
p\left(\bar{r}_{A}\right)=\frac{1}{4 \pi} \int_{S}\left(p(\bar{r}) \frac{1+j k u}{u} \cos \varphi \frac{e^{-j k u}}{u}+j \omega \rho \cdot v_{n}(\bar{r}) \frac{e^{-j k u}}{u}\right) d S
$$


This is the Kirchhoff integral (also called Helmholtz-Kirchhoff integral equation or Helmholtz-Kirchhoff theorem). It states that for a point $A$ in volume $V$ with surface $S$ the pressure can be calculated from the pressure at the surface and a dipole radiation from that surface element $d S$, with its axes normal to the surface (left part) and from the normal velocity at the surface $S$ and a monopole distribution (see also figure 6.5).

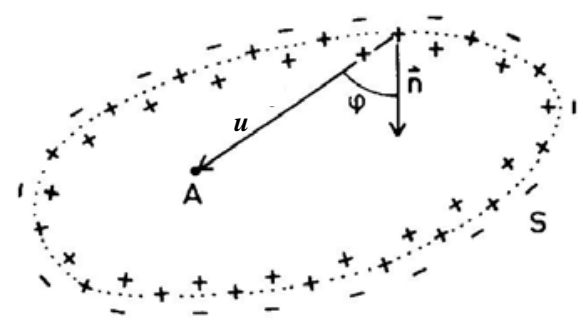

Figure 6.5. Graphic representation of the Kirchhoff integral, the pressure in A results from a contribution from dipole sources and monopole sources, including the directivity (from [Berkhout82]).

To be able to calculate the field inside $V$, the pressure and velocity fields on $S$ must be known. The pressure and velocity can be calculated e.g. for a monopole source outside $V$. However, it is noted that (6.13) is also valid for multiple sources, or more generally, every possible sound field, provided that at every position on $S$ (also at the "rear" side) the pressure and velocity are known.

Taking a monopole source $B$ outside $V$, a spherical sound field is generated. For a point on the surface $S$ at distance $s$ and omitting the time dependence $e^{-i \omega t}$, the pressure $p(\bar{r})$ will be, see also (5.7a):

$$
p(\bar{r})=\hat{p} \frac{e^{-j k s}}{s}
$$

The velocity at this point on $S$ can be calculated from:

$$
v(\bar{r})=-\frac{1}{j \rho \omega} \frac{\partial p}{\partial s}=\frac{1}{\rho c} \frac{1+j k s}{j k s} \hat{p} \frac{e^{-j k s}}{s}
$$

and the normal velocity: $v_{n}(\bar{r})=\cos \alpha \cdot v(\bar{r})$, where $\alpha$ is the angle of the incident wave (here for the normal pointing outward).

If this monopole source $B$ is used outside $V$ and $s$ is defined as the distance between the source and the point on the surface $S$, the pressure in $A$ can be expressed as:

$$
p\left(\bar{r}_{A}\right)=\frac{\hat{p}}{4 \pi} \int_{S}\left(\frac{1+j k u}{u} \cos \varphi \frac{e^{-j k(s+u)}}{s u}+\frac{1+j k s}{s} \cos \alpha \frac{e^{-j k(s+u)}}{s u}\right) d S
$$

For a bounded volume with a closed surface, (6.16) is a rigorous solution. 


\section{$\underline{6.3}$ The exterior problem}

The radiation from the surface to the bounded volume $V$ as shown in figure 6.2 is called the interior problem. Although the basic principle of the Kirchhoff integral, derived from Greens second identity, is basically for a bounded volume, the boundaries can be extended to infinity, which is known as the the exterior problem.

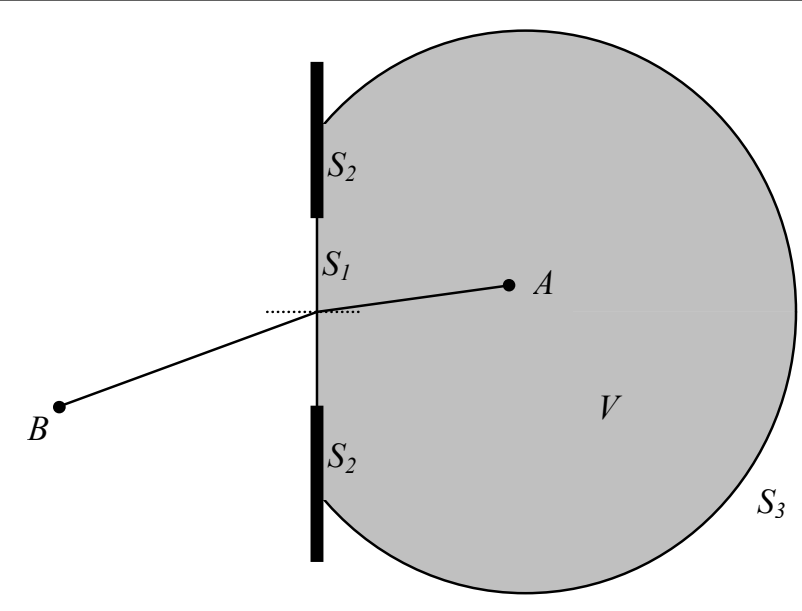

Figure 6.6. Geometry showing the exterior problem with three boundaries: $S_{1}$ exposed, $S_{2}$ not exposed and $S_{3}$ at infinity.

Figure 6.6 shows a situation of an opening in a rigid surface and a volume that is extended to infinity. There are three assumed boundary conditions:

- $\quad S_{I}$ : In this area the pressure and velocity are calculated from the monopole source $B$,

- $S_{2}$ : Directly behind the rigid surface the pressure and velocity is assumed to be zero.

- $\quad S_{3}$ : The boundary at infinite distance does not reflect energy. It can be shown that if the propagating wave fulfills the Sommerfeld radiation conditions that the contribution of this part of the integral in the limit will go to zero (see e.g., [Born59], [Wrobel02]).

In reality especially the areas $S_{1}$ and $S_{2}$ will influence each other. The assumption above on the boundary conditions $S_{1}$ and $S_{2}$ is called the Kirchhoff approximation. It is

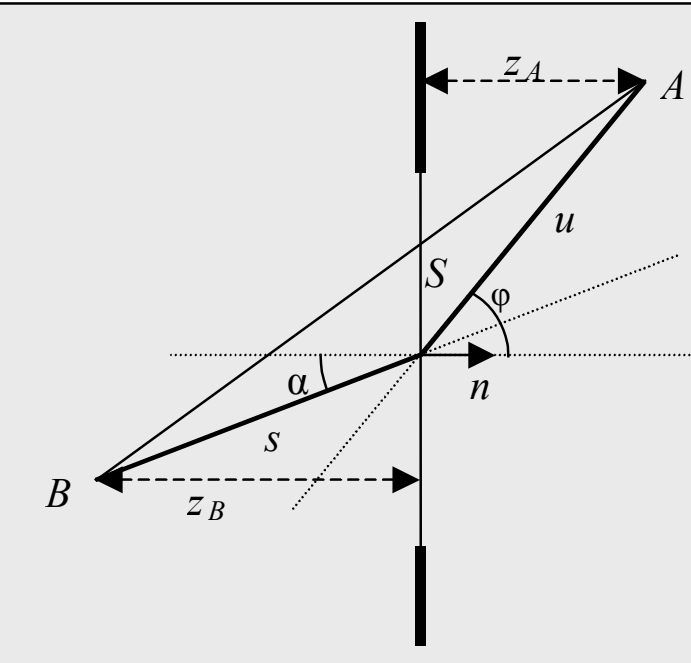

Note: This figure shows the opening in a rigid wall. For each point on the surface $S$ of this opening, there is an angle $\alpha$ at the side of source $B$ and angle $\varphi$ at the side of receiver $A$. If $S$ is a flat surface then:

$$
\cos \alpha=\frac{Z_{B}}{s} \text { and } \cos \varphi=\frac{Z_{A}}{u}
$$

In the more general case the cosine of the angle can be determined from the inner product (with $\bar{a}=$ the vector from the surface to the point and $\bar{n}$ is the normal to the surface):

$$
\cos \varphi=\frac{\bar{a} \cdot \bar{n}}{|\bar{a}| \cdot|\bar{n}|}
$$

Figure 6.7. Geometry of exterior problems with flat surface $S$ 
the basis of Kirchhoff's diffraction theory. With this assumption it is possible to limit the integration with (6.13) or (6.16) to real boundaries and calculate the sound radiation to an unbounded volume.

To derive analytic functions from (6.16) it might be necessary to approximate this function. In the far field $(u>>\lambda, k u>>1)$ and $(s>>\lambda, k s>>1)$ the Fresnel-Kirchhoff diffraction formula will be obtained:

$$
p_{A}=\frac{j \hat{p}}{\lambda} \int_{S}\left(\frac{\cos \alpha+\cos \varphi}{2}\right) \frac{e^{-j k(s+u)}}{s u} d S
$$

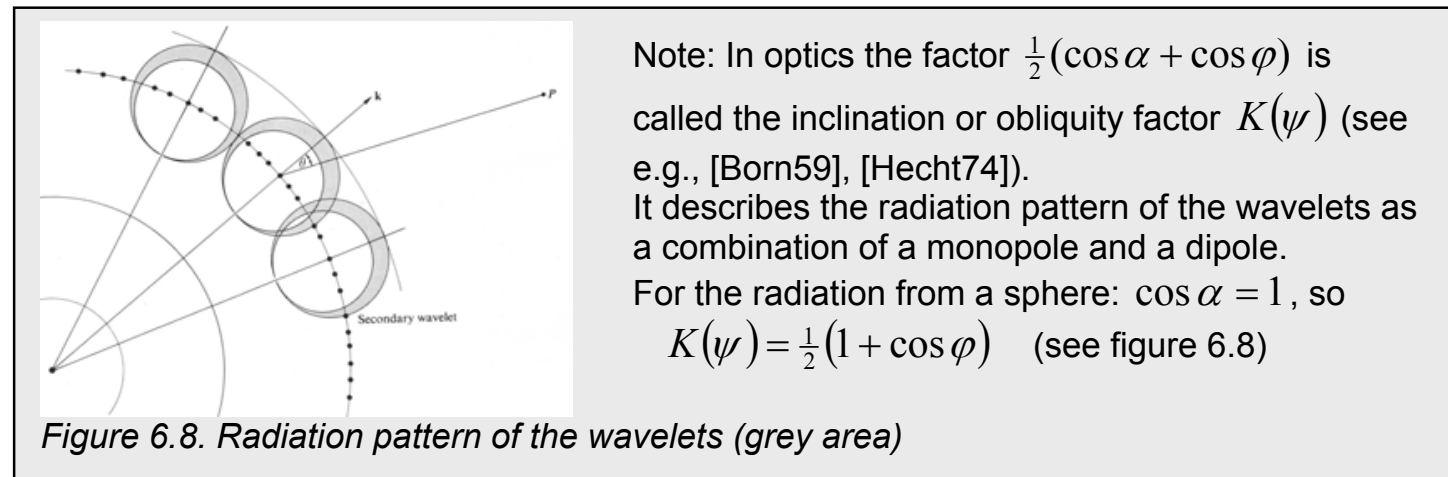

In many publications the inclination or obliquity factor $\frac{1}{2}(\cos \alpha+\cos \varphi)$ is set to 1 . This will further simplify (6.17) and is correct for large plane virtual surfaces (Rayleigh integral). For curved surfaces, (6.16) or (6.17) need to be applied. 


\section{$\underline{6.4 \text { Reflection and scattering }}$}

The formulation (6.13) is based on the homogeneous Helmholtz wave equation (6.2), meaning a source free field inside $V$. In situations with a source inside $V$ and reflections against boundaries, the reflected sound field can be determined by firstly calculating the sound field from the sound source inside $V$ on the boundary and secondly by formulating the correct boundary conditions in terms of pressure and velocity. Formula (6.13) will then provide the scattered sound field.

Obtaining the correct boundary conditions is a difficult task since the pressure at one point of the boundary will influence the pressure on another point and visa versa. By formulating the problem in Matrix notation this problem can be solved by matrix inversion, as done in BEM (Boundary Element Method).

Note: Boundary Element Method (BEM):

BEM is based on the Helmholtz-Kirchhoff integral equation. Including the direct field from the source, the generalised form is (see e.g.,[Cox04], [Kirkup07], [Wrobel02]) :

$$
c\left(r_{A}\right) P\left(r_{A}, \omega\right)=P\left(r_{s}-r_{A}\right)+\frac{1}{4 \pi} \int_{S}\left(P(r, \omega) \frac{\partial G\left(r-r_{A}, \omega\right)}{\partial n}-G\left(r-r_{A}, \omega\right) \frac{\delta P(r, \omega)}{\delta n}\right) d S
$$

with $P\left(r_{s}-r_{A}\right)$ is the pressure at point $A$, directly from the source

for $\quad A$ is within the volume $V: c\left(r_{A}\right)=1$

$A$ is on the surface $S: \quad c\left(r_{A}\right)=\frac{1}{2}$

$A$ is outside volume $V: \quad c\left(r_{A}\right)=0$

This formula can be written in Matrix form (see e.g., [Kirkup07]), by discretisation of the surface $S$. At this surface this will result in:

$$
\left\{\left(M+\frac{1}{2} I\right) p\right\}\left(r_{A}\right)=\{L v\}\left(r_{A}\right)
$$

with

$$
\begin{aligned}
& \{L v\}\left(r_{A}\right)=\int_{S}\left(G\left(r-r_{A}, \omega\right) \frac{\delta P(r, \omega)}{\delta n}\right) d S \\
& \left\{M p\left(r_{A}\right)\right\}=\int_{S} P(r, \omega) \frac{\partial G\left(r-r_{A}, \omega\right)}{\partial n} d S
\end{aligned}
$$

As a first step, the matrix equation at the boundary will be solved. When all pressures and velocities at the boundary are known, the radiation into the volume $V$ can be calculated. Since the correct pressures at the boundary are determined, this method can be considered as a full harmonic solution.

In this study the first Born approximation will be used, which is analogous to the "Kirchhoff approximation" or "tangential plane approximation" in optics (see e.g., [Elmore85], [Yamada05], [Ripoll01]). In (6.13) the pressure and velocity on the boundary $S$ will be based on the incident sound according to (6.14) with a source outside $V$. To be able to calculate the reflection, with the boundary $S$ acting as a reflector, a sound source inside $V$ will be assumed. For the reflected sound wave, the boundary conditions on $S$ will then be assumed to have the same signal but in the opposite direction (same pressure and particle velocity in opposite direction), see figure 6.9. The summated particle velocity of the incident field and reflected field will be zero, as is the case for a perfectly reflecting boundary. The incident pressure gradient will be related to the normal pointing inward. 


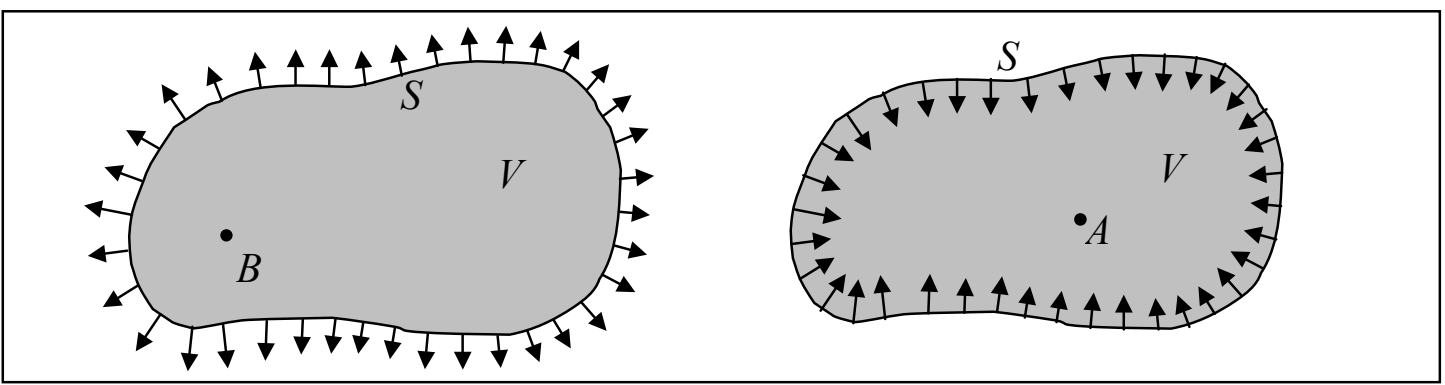

Figure 6.9. Schematic representation of the calculation of the reflected sound field. Left: determination of normal velocities on $S$ due to the source $B$ inside $V$. Right: inversion of the normal velocities on $S$ and calculation of the source free field inside $V$.

The pressure at point $A$, inside $V$, due to the reflection from boundary $S$ (not including the direct field), with the source inside $V$ will be:

$$
p_{A}=\frac{\hat{p}}{4 \pi} \int_{S}\left(\frac{1+j k u}{u} \cos \varphi \frac{e^{-j k(s+u)}}{s u}+\frac{1+j k s}{s} \cos \alpha \frac{e^{-j k(s+u)}}{s u}\right) d S
$$

with $s \neq 0, u \neq 0$

This is actually the same formula as the Kirchhoff integral of (6.16), now with normal pointing inward.

The consequence of the approximation is that a (partial) harmonic solution is obtained, with only the first reflection incorporated. Secondary reflections between different parts of the boundary are not considered. The boundary reflects for the first reflection only and is transparent after this first reflection, see figure 6.10.

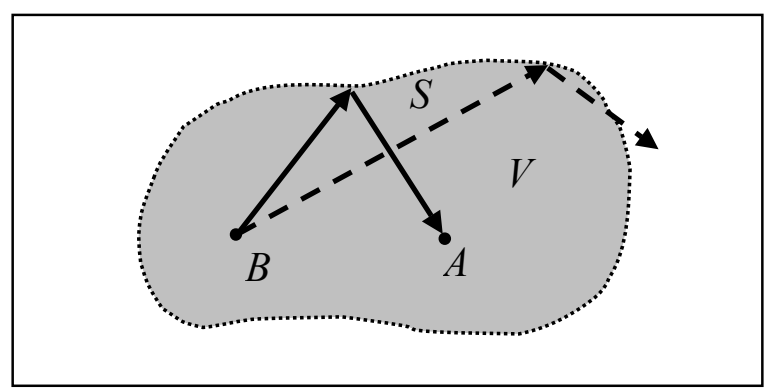

Figure 6.10. The boundary $S$ is reflecting for the first reflection (solid ray) but acoustically transparent for higher order reflections (dashed ray).

For the purpose of this study, which is the approximation of the concentrated sound field in and around the focusing point due to reflections from curved surfaces, the first reflection is of the main interest.

In practical applications second and higher order reflections are mostly reduced in strength due to diffraction and absorption. Moreover, the higher order reflections on the curved surface itself are spread over time and will not cause echo effects. So the alterations of the harmonic sound field in the focusing point due to these multiple reflections are of less practical relevance and it is without objection that these are not considered here. 


\section{$\underline{6.5 \text { Numerical calculations }}$}

By discretisation of (6.18) numerical calculations of the sound field can be performed:

$$
p_{A}=\frac{\hat{p}}{4 \pi} \sum_{S}\left(\frac{1+j k u}{u} \cos \varphi \frac{e^{-j k(s+u)}}{s u}+\frac{1+j k s}{s} \cos \alpha \frac{e^{-j k(s+u)}}{s u}\right) \Delta S
$$

The dimensions of the surface element $\Delta S$ have to be small. Most of the calculations in this study use 8 points per wavelength $(\sqrt{\Delta S} / \lambda)$. Sometimes this number is increased to 12 to obtain a "smoother" curve in the result.

The coordinates of the elements are the points of gravity of the surface elements.

At a short distance from a surface, errors may arise if the distance between the points is small compared to the size of the elements. This will be the case for long, small elements. So the meshing of the surface has to be done in a way that the dimensions of the surface element are in the same order of magnitude. For more complicated structures like spheres and ellipses, this requires special meshing routines.

The meshing depends on the wavelength and therefore on the frequency $f$. For calculating the pressure at a single point, the calculation time relates to $f^{2}$. When calculating the pressure along a line, the required distance between the calculation points on this line is also frequency dependent. So the calculation time of this calculation relates to $f^{3}$. When calculating the pressure in an area, the time dependency is $f^{4}$. Therefore, for the high frequencies there are severe time limitations for the calculation and presentation of the results.

The calculation is done in 3 steps: first meshing the geometry, secondly calculating the incident sound on the surface, and thirdly calculating the pressure in a field point or number of field points. 


\section{SECTION III. APPLICATION OF THE THEORY ON DIFFERENT GEOMETRICAL SHAPES}

The Kirchhoff Integral formulation of (6.18) is the basis for further investigations of the sound field. Whereas the Kirchhoff Integral formulation in (6.18) is a general formulation for each shape, in this section it will be applied to spheres in chapter 7 , cylinders in chapter 8 , ellipsoid shapes in chapter 9 and rectangular, double-curved surfaces in chapter 10.

The emphasis in this study is on the reflected sound field from spheres in chapter 7. In addition to the most relevant situation with the source and receiver in the focusing point or at the focusing line, situations will be investigated that have source positions and/or receiver positions outside the focusing point or line.

In these cases, approximations of the integral are needed, since full analytical solutions of the Kirchhoff Integral are not possible.

\section{Chapter 7}

\section{The sound field in a sphere}

In this chapter the more general formulation of the Kirchhoff integral (6.18) will be applied to the specific situation of reflections from a sphere or sphere segment.

The pressure in the focusing point can be derived directly from the integral formulation (par. 7.2). For a description of the sound field in the area around the focussing point, an approximate solution of the integral will be derived.

\section{$\underline{7.1 \text { Integral formulation for a sphere }}$}

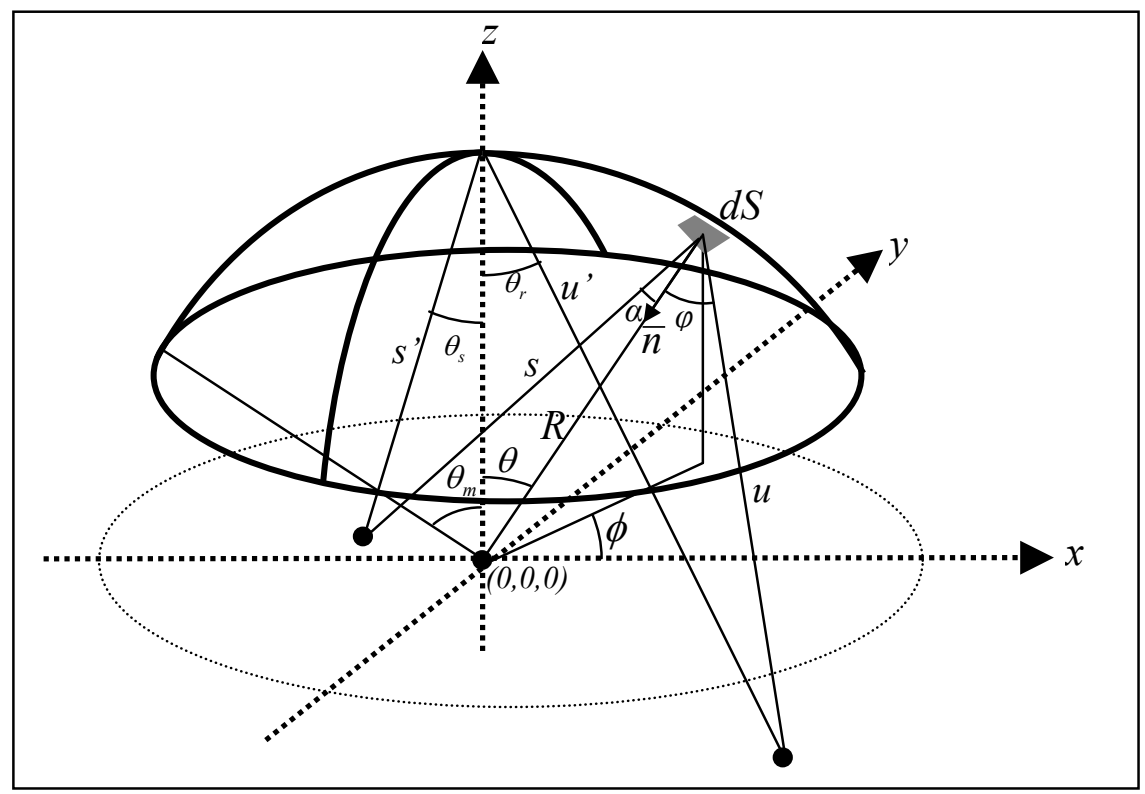

Figure 7.1. Coordinates used for the sphere segment.

The geometry of a spherical reflector with a sound source in $B$ and the receiver in $A$ is shown in figure 7.1. The origin is positioned in the centre of the spherical reflector. Using polar coordinates, positions on the sphere segment with radius $R$ are described by $x=R \sin \theta \cos \phi$ and $y=R \sin \theta \sin \phi, z=R \cos \theta$, a small surface element can be described by $d S=R^{2} \sin \theta d \theta d \phi$ and the integral formulation for the pressure in $A$ of a reflection from this surface becomes: 


$$
p(A, \omega)=\frac{\hat{p} R^{2}}{4 \pi} \int_{\theta=0}^{\theta_{m}} \int_{\phi=0}^{2 \pi} \sin \theta\left(\frac{1+j k u}{u} \cos \varphi+\frac{1+j k s}{s} \cos \alpha\right) \frac{e^{-j k(u+s)}}{s u} d \theta d \phi
$$

Figure 7.2 shows the result of some numeric calculations using (7.1) at the $y=0$ plane (a vertical section of the sphere segment shown in figure 7.1), with the source in the centre of the sphere segment. The results are shown for two frequencies and three aperture angles $\theta_{m}$.

For high frequencies, e.g., situation b) with frequency $1000 \mathrm{~Hz}$ and $\theta_{m}=\frac{1}{5} \pi$, an illuminated region is obtained with strong interferences; for low frequencies and small aperture angles a diverging sound field is obtained, e.g., for situation $f$ ) with frequency $250 \mathrm{~Hz}$ and $\theta_{m}=\frac{1}{10} \pi$.

In the next two paragraphs two specific situations will be discussed: the pressure in the focusing point and the pressure in the far field for small sphere segments.

For other situations, an approximate solution of (7.1) will be derived in chapter 7.4.

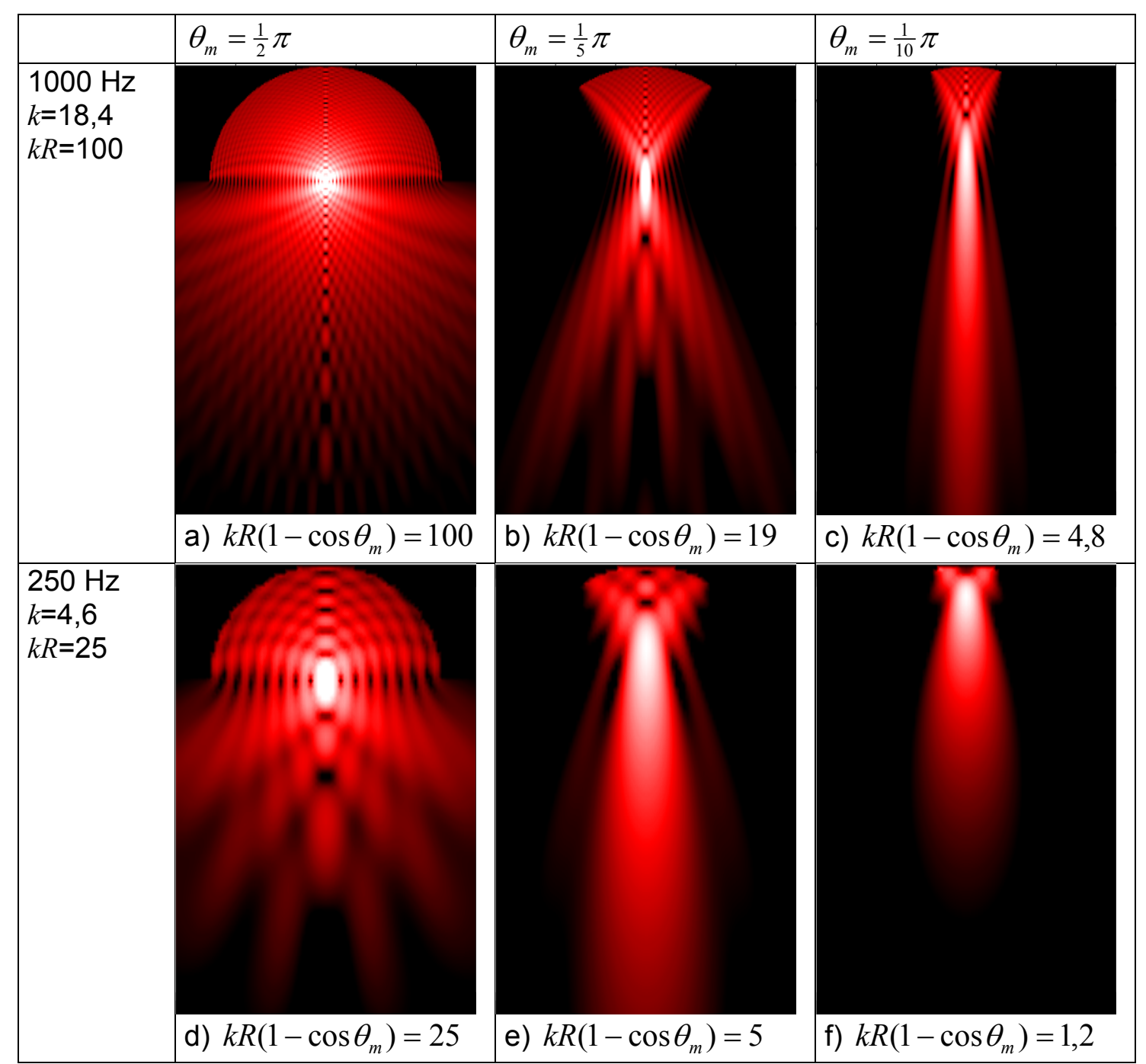

Figure 7.2. Numeric calculations of the pressure of the reflected sound, based on (7.1). For three different sizes of the sphere segment $\left(\theta_{m}=\frac{1}{2} \pi, \frac{1}{5} \pi, \frac{1}{10} \pi\right)$ and two frequencies (250 and $1000 \mathrm{~Hz}$ ). The radius $R$ of the sphere segment is $5,4 \mathrm{~m}$, the area shown is $14 \mathrm{~m} \times 21 \mathrm{~m}$. The source $(\hat{p}=1 \mathrm{~N} / \mathrm{m})$ is in the centre point. Color range: white $=+10 \mathrm{~dB}$, black $=-20 \mathrm{~dB}$. 


\section{$\underline{7.2 \text { Pressure in the focusing point of a sphere }}$}

In case the source is positioned in the centre of the sphere, the focusing point will be in the same point. At that point: $s=u=R, \cos \alpha=\cos \varphi=1$. Assuming $k R>>1$ the pressure in the focusing point reduces to:

$$
p(0, \omega)=\frac{j \hat{p}}{\lambda} \int_{\theta=0}^{\frac{1}{2} \pi} \int_{\phi=0}^{2 \pi} \sin \theta \cdot e^{-j k 2 R} d \phi d \theta=j k \hat{p} \cdot e^{-j k 2 R}
$$

For sphere segments with opening angle $\theta_{m}$ (for the hemisphere $\theta_{m}=\frac{1}{2} \pi$ ) the pressure will be:

$$
p(0, \omega)=j k \hat{p}\left(1-\cos \theta_{m}\right) \cdot e^{-j k 2 R}
$$

The amplitude of the pressure will be:

$$
|p(0, \omega)|=k \hat{p}\left(1-\cos \theta_{m}\right)
$$

The rms value will be:

$$
p_{r m s}^{2}=\frac{1}{2} \hat{p}^{2} k^{2}\left(1-\cos \theta_{m}\right)^{2}
$$

This pressure is not dependent on the radius of the concave element, only on opening angle and frequency.

With these results the maximum sound pressure in the focusing point, due to the first reflection from a spherically curved surface, can be calculated. The result is linearly dependent on frequency. High frequencies give very strong amplification in the focusing point. It is important to note here that a perfect spherically curved surface and a full reflection is assumed.

Note: For a full sphere $\left(\theta_{m}=\pi\right)$, from (7.3a), the pressure will be: $|p(0, \omega)|=2 k \hat{p}$

In [Mechel09] a different formula is derived for the pressure in the centre of a hemisphere with a reflecting floor, which is similar to the full sphere:

Mechel assumes the sound field in the sphere to be a spherical Bessel function:

$$
p(r)=a j_{0}(k r)
$$

with $\quad j_{0}(k r)=\sin (k r) / k r$ and

$r$ is the distance from the centre.

By setting the velocity to zero at the boundary, the amplitude $a$ of this spherical Bessel function is calculated to be:

$$
a=-P_{Q} \frac{h_{1}^{(2)}(k R)}{j_{1}(k R)}=-P_{Q} \frac{j_{1}(k R)-i y_{1}(k R)}{j_{1}(k R)}
$$

with $\quad j_{1}(x)=\frac{\sin x}{x^{2}}-\frac{\cos x}{x}$ and

$$
y_{1}(x)=-\frac{\cos x}{x^{2}}-\frac{\sin x}{x}
$$

This is a highly oscillating function that depends on frequency and radius, with singularities for $j_{1}(k R)=0$; it is clearly different from the solution $|p(0, \omega)|=2 k \hat{p}$, which is not dependent on the radius of the sphere. 
The difference is caused by the multiple reflections that are incorporated in the formulation of Mechel. In the full harmonic solution, multiple reflections will interfere with each other and may generate a further amplification or extinction of the pressure.

In the approximation in this study, only the first reflection is considered (see par. 6.3). This will generally be sufficient, especially in halls with absorbing floor (audience) instead of a reflecting floor, as assumed by Mechel. And even with a reflecting floor, the time domain solution will be more logical than the full harmonic solution, since repetitive reflections will occur. The determination of the reflection strength of the first reflection will be the starting point for that analysis.

For the description of the sound field, the pressure at a certain point $(A)$ will be related to the pressure in the focusing point:

$$
f(A)=\frac{p(A, \omega)}{p(0, \omega)}=\frac{p(A, \omega)}{\hat{p} k\left(1-\cos \theta_{m}\right)}
$$




\section{$\underline{7.3 \text { Pressure in the far field for small aperture angle }}$}

If $\theta_{m}$ is reduced, the curvature of the dome segment will be less effective at concentrating the sound field. Figure $7.2 f$ shows a diverging sound field for $k R\left(1-\cos \theta_{m}\right)=1,2$.

In figure 7.3 the reflected sound pressure of a dome segment is compared to the reflected pressure of a flat circular disk with the same $\theta_{m}$. For $\theta_{m}=\frac{1}{32} \pi$ and $\mathrm{k}=10$ there is little difference. For this frequency the width of the disk is little more than $3 \lambda$ and the depth of the curvature at the edges is $R \cos \left(1-\theta_{m}\right)=0,05 \mathrm{~m}$, less than $0,1 \lambda$.

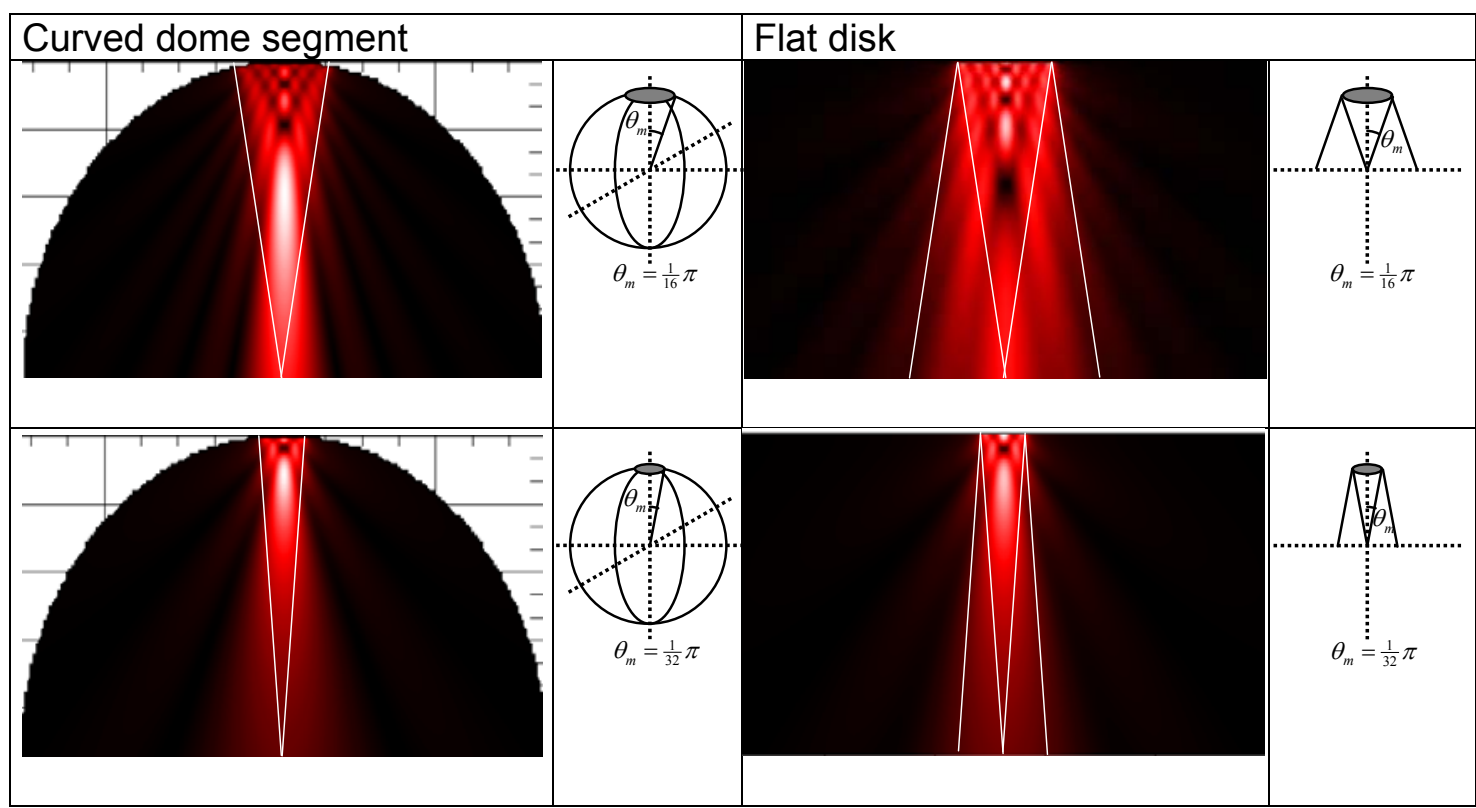

Figure 7.3. Reflected sound pressure from a sphere segment and a flat disk; $R=10 \mathrm{~m}, k=10$ $(f=500 \mathrm{~Hz}), \hat{p}=1 \mathrm{~N} / \mathrm{m}$, Color range: linear, black $=0$, white $\geq 1 \mathrm{~Pa}$.

Figure 7.4 shows the pressure along the $x$-axis (for $z=0$, so through the centre) of the flat circular disk and the dome segment for $\theta_{m}=\frac{1}{32} \pi(k=10, R=10)$. It shows that there is little difference.

Also included in this figure is the theoretical solution for the far field radiation of a small circular disk. This solution presumes the radius $a<<R$ and a plane surface wave incident on the disk. This solution is known as the Fraunhofer diffraction, for the case of a circular disk also called "Airy pattern" (see e.g., [Hecht74], [Born59]). This pressure at distance $R$ from the disk is:

$$
p=\left|\frac{k \hat{p} a^{2}}{R} \cdot \frac{J_{1}\left(\frac{k a x}{R}\right)}{\frac{k a x}{R}}\right|
$$

where: $x=$ the distance along the $x$-axis and

$J_{1}()=$ the Bessel function of the first kind with order 1. 


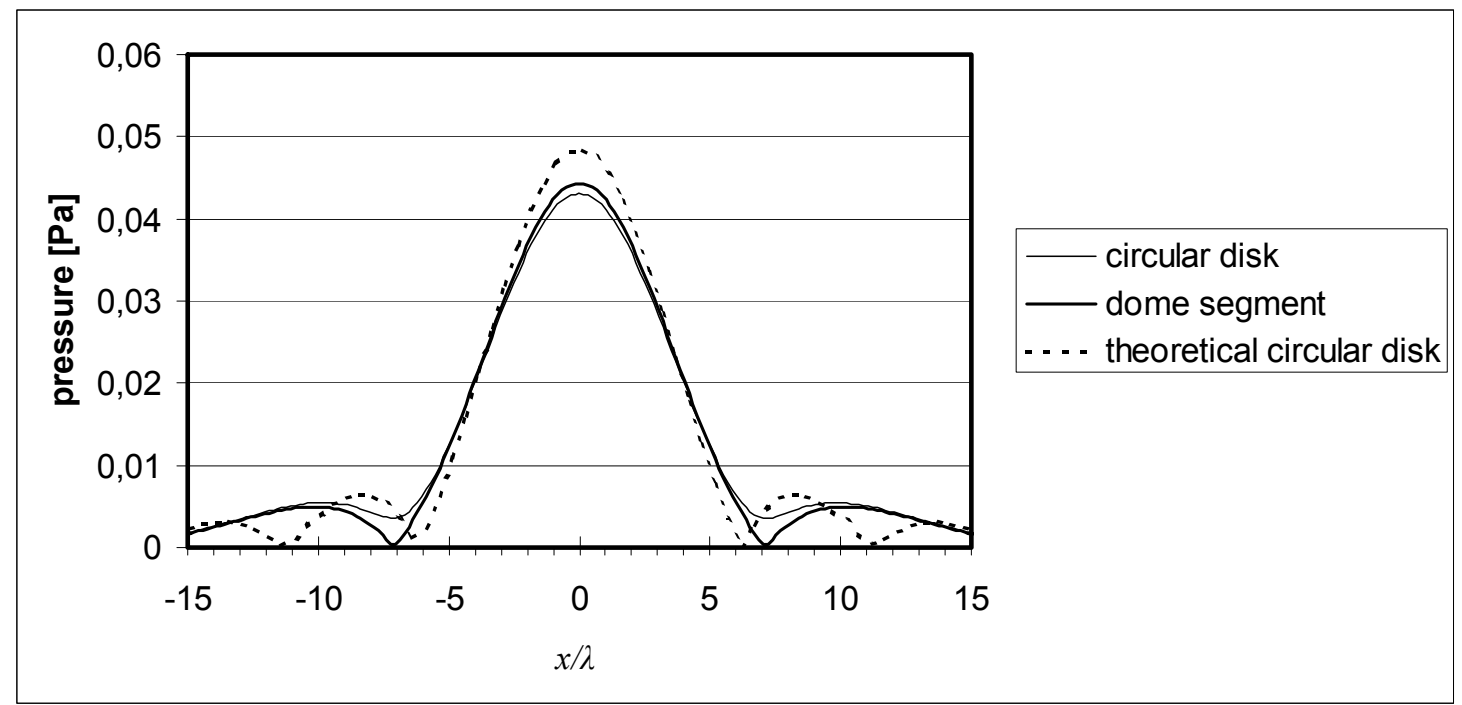

Figure 7.4. Reflected sound pressure at $z=0$ from a dome segment, $\theta_{m}=\pi / 32$, a flat circular disk and the theoretical solution for a flat circular disk in the far field (with plane incident wave); $R=10 \mathrm{~m}, k=10(f \sim 500 \mathrm{~Hz}), \hat{p}=1 \mathrm{~N} / \mathrm{m}$, as a function of the relative distance $x / \lambda$.

It can be concluded that for shallow curved segments (the "depth" of the segment $\left.R \cos \left(1-\theta_{m}\right)<<\frac{1}{4} \lambda\right)$ the reflected sound field is diverging and can be accurately calculated with the Fraunhofer diffraction. 


\subsection{Approximation of the sound field in a sphere}

To solve the integral formulation for a circular sphere segment (7.1) with opening angle $\theta_{m}$, for source and/or receiver positions other than the centre of the sphere, the distances $s$ and $u$ have to be expressed in terms of the integration angles $\theta$ and $\phi$. This will result in expressions containing a root in the phase and amplitude. There are no analytical solutions for this kind of integrals. Therefore, approximations are necessary. The obvious approximation is to apply the Taylor series for the root:

$$
\sqrt{1+x}=1+\frac{x}{2}-\frac{x^{2}}{8}+\ldots
$$

The accuracy of this approximation improves for smaller $x$. The strategy would be to have a first "estimate", independent of the angles $\theta$ and $\phi$, that is as large as possible and second variable, as small as possible, that is dependent on the angles $\theta$ and $\phi$.

The approximation and solution technique used here will follow the strategy used in [Chen93]. Additionally the incident sound will be incorporated (in [Chen93] only the in-phase radiation from a piezoelectric element is considered) and larger aperture angles will be considered.

\subsubsection{Approximate solution of the integral}

The geometry of the problem is indicated in figure 7.1 .

The coordinates for the source $B$ are: $\left(x_{B}, y_{B} . z_{B}\right)$ and for the receiver $A$ : $\left(x_{A}, y_{B}, z_{B}\right)$.

The positions on the sphere segment are described by $z=R \cos \theta, x=R \sin \theta \cos \phi$ and $y=R \sin \theta \sin \phi$.

The first step is to approximate the distances $s$ and $u$ from source and receiver respectively to surface element $d S$, as a function of the angles $\theta$ and $\phi$.

Derivation:

The distance from the source $B$ to the surface element $d S$ is described by:

$$
\begin{aligned}
s^{2} & =\left(R \sin \theta \cos \phi-x_{B}\right)^{2}+\left(R \sin \theta \sin \phi-y_{B}\right)^{2}+\left(R \cos \theta-z_{B}\right)^{2} \\
& =R^{2}-2 R \sin \theta\left(x_{B} \cos \phi+y_{B} \sin \phi\right)-2 z_{B} R \cos \theta+y_{B}{ }^{2}+x_{B}{ }^{2}+z_{B}{ }^{2}
\end{aligned}
$$

The best possible approximation of the distance, independent of $\varphi$ or $\theta$, will be the distance from $B$ to the middle of the sphere segment $s^{\prime}$ (see figure 7.1):

$$
s^{\prime 2}=\left(R-z_{B}\right)^{2}+x_{B}{ }^{2}+y_{B}{ }^{2}=R^{2}-2 z_{B} R+z_{B}{ }^{2}+x_{B}{ }^{2}+y_{B}{ }^{2},
$$

This will result in:

$$
s^{2}=s^{\prime 2}-2 R \sin \theta\left(x_{B} \cos \phi+y_{B} \sin \phi\right)+2 z_{B} R(1-\cos \theta)
$$


In the same way the distance from $d S$ to the receiver in $A$ can be determined:

$$
u^{2}=u^{\prime 2}-2 R \sin \theta\left(x_{A} \cos \phi+y_{A} \sin \phi\right)+2 z_{A} R(1-\cos \theta)
$$

The first approximation to make is taking the first two terms of the Taylor series.

$$
\begin{aligned}
& u \approx u^{\prime}-\frac{R}{u^{\prime}} \sin \theta\left(x_{A} \cos \phi+y_{A} \sin \phi\right)+\frac{R}{u^{\prime}} z_{A}(1-\cos \theta) \text { and } \\
& s \approx s^{\prime}-\frac{R}{s^{\prime}} \sin \theta\left(x_{B} \cos \phi+y_{B} \sin \phi\right)+\frac{R}{s^{\prime}} z_{B}(1-\cos \theta)
\end{aligned}
$$

This approximation is valid if the third term fulfills the following condition (for the receiver):

$$
\begin{aligned}
& \varepsilon<k \frac{\left(-2 R \sin \theta\left(x_{A} \cos \phi+y_{A} \sin \phi\right)+2 z_{A} R(1-\cos \theta)\right)^{2}}{8 u^{\prime 3}}<\frac{2 \pi}{8}, \text { so } \\
& \sin ^{2} \theta\left(x_{A} \cos \phi+y_{A} \sin \phi\right)^{2}-2 z_{A}(1-\cos \theta) \sin \theta\left(x_{A} \cos \phi+y_{A} \sin \phi\right)+z_{A}{ }^{2}(1-\cos \theta)^{2} \\
& <\left(x_{A}+y_{A}\right)^{2}-2 z_{A}\left(x_{A}+y_{A}\right)+z_{A}{ }^{2}<r_{A}{ }^{2}<\frac{\lambda R}{4}
\end{aligned}
$$

where $r_{A}$ is distance of $A$ to the centre.

So the approximation is valid for:

$$
r_{A}<\frac{1}{2} \sqrt{\lambda R}
$$

For a distance of the source $B$ to the centre $r_{B}<\frac{1}{2} \sqrt{\lambda R}$ and a distance of the receiver $A$ to the centre $r_{A}<\frac{1}{2} \sqrt{\lambda R}$, the distances $s$ and $u$ may be approximated, based on the distances to the middle of the sphere segment:

$$
\begin{aligned}
& u \approx u^{\prime}-\frac{R}{u^{\prime}} \sin \theta\left(x_{A} \cos \phi+y_{A} \sin \phi\right)+\frac{R}{u^{\prime}} z_{A}(1-\cos \theta) \text { and } \\
& s \approx s^{\prime}-\frac{R}{s^{\prime}} \sin \theta\left(x_{B} \cos \phi+y_{B} \sin \phi\right)+\frac{R}{s^{\prime}} z_{B}(1-\cos \theta)
\end{aligned}
$$

The second approximation is, using (7.1), only to consider the far field $(k u>>1$ and $k s>>1)$. Thirdly a position of the source and receiver not far from the centre of the sphere segment can be assumed, resulting in the assumption $\cos \alpha=\cos \varphi=1$ :

$$
p(A, \omega)=j \frac{\hat{p} k R^{2}}{2 \pi} \int_{\theta=0}^{\theta_{m}} \int_{\phi=0}^{2 \pi} \sin \theta \frac{e^{-j k(u+s)}}{s u} d \theta d \phi
$$

Next step is to use the expressions for $s$ and $u$ from (7.11) in (7.13). 
Derivation: Substituting the obtained approximations for $u$ and $s$ (for the amplitude use $u$ ' and $\left.s^{\prime}\right)$ in (7.13):

$$
\begin{aligned}
& p(A, \omega)=j \frac{\hat{p} k R^{2} e^{-j k\left(u^{\prime}+s^{\prime}\right)}}{2 \pi u^{\prime} s^{\prime}} . \\
& \int_{\theta=0}^{\theta_{m}} \sin \theta e^{-j k R\left(\frac{z_{A}}{u^{\prime}}+\frac{z_{B}}{s^{\prime}}\right)(1-\cos \theta)} \int_{\phi=0}^{2 \pi} e^{j k R \sin \theta\left(\left(\frac{x_{A}}{u^{\prime}}+\frac{x_{B}}{s^{\prime}}\right) \cos \phi+\left(\frac{y_{B}}{s^{\prime}}+\frac{y_{A}}{u^{\prime}}\right) \sin \phi\right)} d \theta d \phi
\end{aligned}
$$

Using $\frac{1}{2 \pi} \int_{0}^{2 \pi} e^{j z(a \cos \phi+b \sin \phi)} d \phi=J_{o}\left(z \sqrt{a^{2}+b^{2}}\right)$,

with $J_{o}$ is the 0 -th order Bessel function of the first kind:

$$
\begin{aligned}
& p(A, \omega)=j \frac{\hat{p} k R^{2} e^{-j k\left(u^{\prime}+s^{\prime}\right)}}{u^{\prime} s^{\prime}} . \\
& \int_{\theta=0}^{\theta_{m}} \sin \theta e^{-j k R\left(\frac{z_{A}}{u^{\prime}}+\frac{z_{B}}{s^{\prime}}\right)(1-\cos \theta)} J_{o}\left(k R \sin \theta \sqrt{\left(\frac{x_{A}}{u^{\prime}}+\frac{x_{B}}{s^{\prime}}\right)^{2}+\left(\frac{y_{B}}{s^{\prime}}+\frac{y_{A}}{u^{\prime}}\right)^{2}}\right) d \theta
\end{aligned}
$$

Substituting $w=\frac{\sin \theta}{\sin \theta_{m}}$ and $\frac{d w}{d \theta}=\frac{\cos \theta}{\sin \theta_{m}}$ results in:

$$
\begin{aligned}
& \begin{array}{l}
p(A, \omega)=j \frac{\hat{p} k R^{2} e^{-j k\left(u^{\prime}+s^{\prime}\right)}}{u^{\prime} s^{\prime}} \\
\qquad \int_{w=0}^{1} \frac{\sin ^{2} \theta_{m} w}{\cos \theta} e^{-j k \sin ^{2} \theta_{m} \frac{R}{2}\left(\frac{z_{A}}{u^{\prime}}+\frac{z_{B}}{s^{\prime}}\right) w^{2}} J_{o}\left(k R \sin \theta_{m} w \sqrt{\left(\frac{x_{A}}{u^{\prime}}+\frac{x_{B}}{s^{\prime}}\right)^{2}+\left(\frac{y_{B}}{s^{\prime}}+\frac{y_{A}}{u^{\prime}}\right)^{2}}\right) d w(7.17) \\
\text { For small } \theta \text { this can be simplified into: } \\
\qquad p(A, \omega)=j \frac{\hat{p} R \cdot e^{-j k\left(u^{\prime}+s^{\prime}\right)}}{\left(s^{\prime} z_{A}+u^{\prime} z_{B}\right)} Y \int_{w=0}^{1} w e^{-j \frac{Y}{2} w^{2}} J_{o}(Z w) d w \\
\text { with } Y=k R \sin ^{2} \theta_{m}\left(\frac{z_{A}}{u^{\prime}}+\frac{z_{B}}{s^{\prime}}\right) \text { and } Z=k R \sin \theta_{m} \sqrt{\left(\frac{x_{A}}{u^{\prime}}+\frac{x_{B}}{s^{\prime}}\right)^{2}+\left(\frac{y_{B}}{s^{\prime}}+\frac{y_{A}}{u^{\prime}}\right)^{2}}
\end{array}
\end{aligned}
$$

The double integral is now reduced to a single integral of a Bessel function:

$$
p(A, \omega)=j \frac{\hat{p} R \cdot e^{-j k\left(u^{\prime}+s^{\prime}\right)}}{\left(s^{\prime} z_{A}+u^{\prime} z_{B}\right)} I(Y, Z)
$$

with:

$$
\begin{aligned}
& I(Y, Z)=Y \int_{w=0}^{1} w e^{-j \frac{Y}{2} w^{2}} J_{o}(Z w) d w, \\
& Y=k R \sin ^{2} \theta_{m}\left(\frac{z_{A}}{u^{\prime}}+\frac{z_{B}}{s^{\prime}}\right) \text { and } \\
& Z=k R \sin \theta_{m} \sqrt{\left(\frac{x_{A}}{u^{\prime}}+\frac{x_{B}}{s^{\prime}}\right)^{2}+\left(\frac{y_{B}}{s^{\prime}}+\frac{y_{A}}{u^{\prime}}\right)^{2}}
\end{aligned}
$$


Note: In [Chen93] the radiation of a spherical piezoelectric radiator is described. The radiation of the surface of this radiator has equal phase. So this corresponds to the situation with a sound source in the origin and full reflection against the surface.

For small $\theta$ and $x_{B}=y_{B}=z_{B}=0$ :

$$
Y=k R \sin ^{2} \theta_{m}\left(\frac{z_{A}}{u^{\prime}}\right) \text { and } Z=k R \sin \theta_{m} \sqrt{\left(\frac{x_{A}}{u^{\prime}}\right)^{2}+\left(\frac{y_{A}}{u^{\prime}}\right)^{2}}
$$

This single integral can be solved by a power series solution (Lommel integrals, see text block, see also [Born59]).

$$
\begin{aligned}
& I(Y, Z)=e^{-j \frac{Y}{2}}\left(u_{1}(Y, Z)+j u_{2}(Y, Z)\right) \\
& \text { with } \begin{aligned}
u_{1}(Y, Z) & =\sum_{n=0}^{\infty}(-1)^{n}\left(\frac{Y}{Z}\right)^{2 n+1} J_{2 n+1}(Z) \\
u_{2}(Y, Z) & =\sum_{n=0}^{\infty}(-1)^{n}\left(\frac{Y}{Z}\right)^{2 n+2} J_{2 n+2}(Z)
\end{aligned}
\end{aligned}
$$

In the shadow zone (for $Y<Z$ ) these series will converge.

In the illuminated zone (where $Z<Y$ ) a better convergence is realized by using:

$$
\begin{aligned}
u_{1}(Y, Z) & =\sin \left(\frac{Y}{2}+\frac{Z^{2}}{2 Y}\right)-v_{1}(Y, Z) \\
u_{2}(Y, Z) & =-\cos \left(\frac{Y}{2}+\frac{Z^{2}}{2 Y}\right)+v_{0}(Y, Z) \\
\text { with } \quad v_{0}(Y, Z) & =\sum_{n=0}^{\infty}(-1)^{n}\left(\frac{Z}{Y}\right)^{2 n} J_{2 n}(Z) \\
v_{1}(Y, Z) & =\sum_{n=0}^{\infty}(-1)^{n}\left(\frac{Z}{Y}\right)^{2 n+1} J_{2 n+1}(Z)
\end{aligned}
$$

For practical applications using the first term of $v_{0}$ and $v_{1}$ will be sufficient:

$$
I(Y, Z)=e^{-j \frac{Y}{2}}\left(\sin \left(\frac{Y}{2}+\frac{Z^{2}}{2 Y}\right)-\left(\frac{Z}{Y}\right) J_{1}(Z)+j\left(-\cos \left(\frac{Y}{2}+\frac{Z^{2}}{2 Y}\right)+J_{o}(Z)\right)\right)
$$

This will result in the following expression for the pressure in $A$ :

$$
|p(A, \omega)|=\frac{\hat{p} R}{\left(s^{\prime} z_{A}+u^{\prime} z_{B}\right)}|I(Y, Z)|
$$

with $|I(Y, Z)|=\left\{\left(J_{o}(Z)-\cos \left(\frac{Y}{2}+\frac{Z^{2}}{2 Y}\right)\right)^{2}+\left(\frac{Z}{Y} J_{1}(Z)-\sin \left(\frac{Y}{2}+\frac{Z^{2}}{2 Y}\right)\right)^{2}\right\}^{\frac{1}{2}}$

and $\quad Y$ and $Z$ as indicated in (7.18).

The pressure in $A$, related to the pressure in the focusing point will be:

$$
f(A)=\frac{R}{k\left(1-\cos \theta_{m}\right)\left(s^{\prime} z_{A}+u^{\prime} z_{B}\right)}|I(Y, Z)|
$$

Based on (7.22), the sound field in the focusing point, on the focusing axis, in the focusing plane and in the far field will be described in the following paragraphs. 


\subsubsection{Pressure in the focusing point based on the approximation}

The pressure in the focusing point will be considered, both for the situation with the source in the centre of the sphere and in the situation with the source outside the centre. For the source in the centre the approximation is expected to give the same result as the direct solution presented in (7.3).

\section{Source in the centre}

At the focusing point, with the source in the centre, both $Y$ and $Z$ will be zero.

Due to the singularity the pressure in the centre can not be calculated using (7.22), and (7.18) will be used:

$$
\begin{aligned}
p(A, \omega) & =j \frac{\hat{p} k R^{2} \sin ^{2} \theta_{m} e^{-j k(2 R)}}{R^{2}} \int_{w=0}^{1} w J_{o}(0) d w \\
& =j \hat{p} k \frac{\sin ^{2} \theta_{m}}{2} e^{-j 2 k R}
\end{aligned}
$$

Since $\frac{1}{2} \sin ^{2} \theta_{m} \approx\left(1-\cos \theta_{m}\right)$ for small $\theta_{m}$, this yields the same as (7.3).

\section{Source position outside the centre}

From the definition of $Y$ and $Z$ it can be seen directly that they both will be zero (and the focusing effect will be maximum) when:

$$
\frac{x_{A}}{u^{\prime}}=-\frac{x_{B}}{s^{\prime}}, \frac{y_{B}}{s^{\prime}}=-\frac{y_{A}}{u^{\prime}} \text { and } \frac{z_{A}}{u^{\prime}}=-\frac{z_{B}}{s^{\prime}} .
$$

These relations correspond to the thin lens formula.

However, it should be noted that there have been some approximations made. In the integration the terms $\cos \alpha$ and $\cos \varphi$ (see formula (7.1)) should be considered. For $z_{A}=z_{B}=0$ the effect of incorporating this factor in the integration can be approximated by (pressure relative to the pressure in the focussing point with the source in the centre):

$$
f\left(-x_{B}\right) \approx 1-\frac{1}{2} \frac{x_{B}^{2}+y_{B}^{2}}{R^{2}} .
$$

Additionally, changing the angle influences the distances $u$ and $s$ due to spherical aberration. The effect can be approximated by taking into account the third term of the Taylor series.

Including the third term of the Taylor series, but still leaving out all terms with $x_{B}{ }^{4}$ or higher order, the distances $s$ and $u$ can be written:

$$
\begin{aligned}
& s \approx R-x_{B} \sin \theta \cos \phi+\frac{x_{B}{ }^{2}}{2 R}-\frac{x_{B}{ }^{2} \sin ^{2} \theta \cos ^{2} \phi}{2 R}+\frac{x_{B}{ }^{3} \sin \theta \cos \phi}{2 R^{2}} \\
& u \approx R+x_{B} \sin \theta \cos \phi+\frac{x_{B}{ }^{2}}{2 R}-\frac{x_{B}{ }^{2} \sin ^{2} \theta \cos ^{2} \phi}{2 R}-\frac{x_{B}{ }^{3} \sin \theta \cos \phi}{2 R^{2}} \\
& \text { and } u+s \approx 2 R+\frac{x_{B}{ }^{2}\left(1-\sin ^{2} \theta \cos ^{2} \phi\right)}{R}
\end{aligned}
$$


The reduction factor of the pressure in the receiver point can be approximated by:
$f\left(-x_{B}\right)=\frac{1}{2 \pi\left(1-\cos \theta_{m}\right)} \int_{0}^{2 \pi \theta_{m}} \sin \theta \cdot e^{-j k \frac{x_{B}{ }^{2}\left(1-\sin ^{2} \theta \cos ^{2} \phi\right)}{R}} d \theta d \phi$, or
$f\left(-x_{B}\right)=\frac{e^{-j k \frac{x_{B}{ }^{2}}{R}}}{2 \pi\left(1-\cos \theta_{m}\right)} \int_{0}^{2 \pi \theta_{m}} \int_{0} \sin \theta \cdot e^{j k \frac{x_{B}{ }^{2}}{R} \sin ^{2} \theta \cos ^{2} \phi} d \theta d \phi$
using $\frac{1}{2 \pi} \int_{0}^{2 \pi} e^{j z \cos ^{2} \phi} d \phi=J_{o}\left(\frac{z}{2}\right)$ will result in:
$f\left(-x_{B}\right)=\frac{e^{-j k \frac{x_{B}{ }^{2}}{R}}}{\left(1-\cos \theta_{m}\right)} \int_{0}^{\theta_{m}} \sin \theta \cdot J_{o}\left(\frac{\pi x_{B}{ }^{2}}{\lambda R} \sin ^{2} \theta\right) d \theta$

This results in the following single integral for the reduction of the pressure at a position $-x_{B}$, relative to the maximum pressure with the source in the centre $\left(-x_{B}=0\right)$.

$$
f\left(-x_{B}\right)=\frac{e^{-j k \frac{x_{B}{ }^{2}}{R}}}{\left(1-\cos \theta_{m}\right)} \int_{0}^{\theta_{m}} \sin \theta \cdot J_{o}\left(\frac{\pi x_{B}{ }^{2}}{\lambda R} \sin ^{2} \theta\right) d \theta
$$

These effects are not incorporated into the approximate solution in (7.18), the validity of that approximation is limited to $x<\frac{1}{2} \sqrt{\lambda R}$, see (7.11).

The reduction factor according to (7.31), as a function of the relative distance $x / \sqrt{\lambda R}$ of source and receiver to the centre (with $x=x_{A}=-x_{B}$ ), is illustrated in figure 7.5 for a hemisphere. Also shown is the numerical solution, which is obtained from several calculations with different $\lambda$ and $R$ (with $R \cos \left(1-\theta_{m}\right)>\lambda$ ). The results show that the approximation of (7.31) is very close to the numerical solution for $x<0,85 \sqrt{\lambda R}$.

For a hemisphere $\left(\theta_{m}=\pi / 2\right)$ and $x<0,85 \sqrt{\lambda R}$ the reduction function may be estimated by:

$$
f(x) \approx \cos \left(\frac{\pi x^{2}}{2 \lambda R}\right)
$$

The reduction effect is less pronounced for smaller opening angles $\theta_{m}$ (see note). 


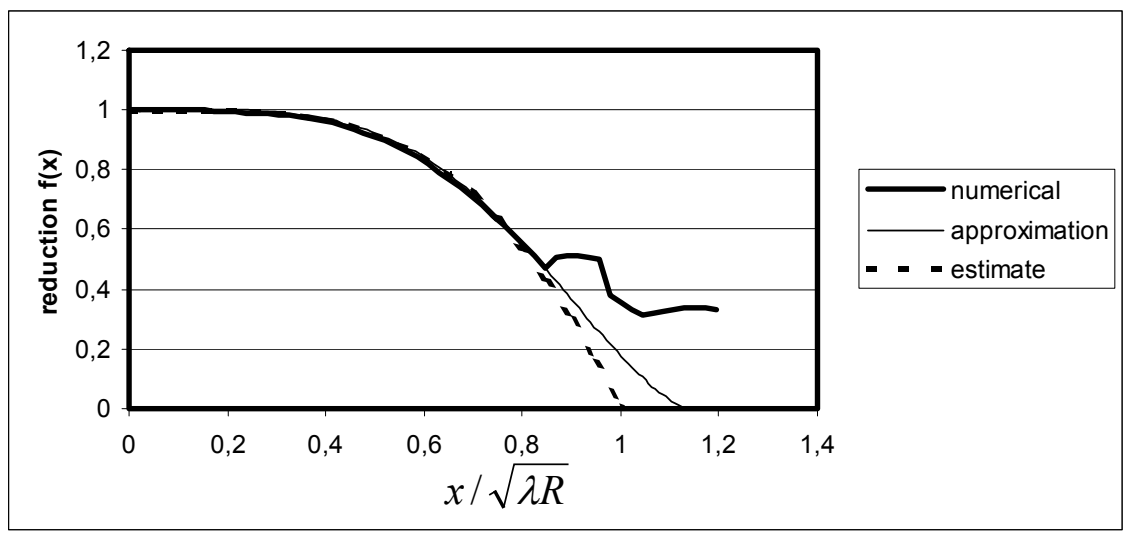

Figure 7.5. The reduction factor $f(x)$ of the pressure in the focusing point depending on the relative distance $x / \sqrt{\lambda R}$ of source and receiver from the centre of the hemisphere. Numerical solution with (7.1) for $\theta_{m}=\pi / 2$, the approximation with (7.31) (by numerical integration) and estimate with (7.32a)

Note: From numerical experiments it was found that for $x<0,85 \sqrt{\lambda R}$ and $R \cos \left(1-\theta_{m}\right)>\lambda$ a fair approximation of the reduction factor can be found, for opening angles smaller than $\theta_{m}=\pi / 2$ :

$$
f(x) \approx \cos \left(\theta_{m} \frac{x_{B}{ }^{2}}{\lambda R}\right)
$$




\subsubsection{Pressure at the axis of the sphere segment}

The pressure along the axis of the sphere segment, through the focusing point will be considered here, again both for the situation with the source at the centre of the sphere and in the situation with the source outside the centre.

\section{Source in the centre}

First, the source will be left in the centre of the sphere while considering the pressure at position $\left(0,0, z_{A}\right)$ along the $z$-axis.

This means, according to (7.18) the parameters $Z$ and $Y$ will be:

$$
\begin{aligned}
& Z=0 \text { and } \\
& Y=k R\left|\frac{z_{A}}{R-z_{A}}\right| \sin ^{2} \theta_{m} .
\end{aligned}
$$

Using (7.22) with $v_{o}=1$ and $v_{1}=0$ the integral $I(Y, Z)$ will be:

$$
\begin{aligned}
& |I(Y, Z)|=\left\{\left(1-\cos \left(\frac{Y}{2}\right)\right)^{2}+\left(\sin \left(\frac{Y}{2}\right)\right)^{2}\right\}^{\frac{1}{2}} \\
& =\sqrt{2} \sqrt{1-\cos \left(k R\left|\frac{z_{A}}{R-z_{A}}\right| \frac{\sin ^{2} \theta_{m}}{2}\right)}
\end{aligned}
$$

This will result in a pressure in $A$ :

$$
|p(A, \omega)|=\frac{\hat{p} R}{\left(s^{\prime} z_{A}+u^{\prime} z_{B}\right)}|I(Y, Z)|=\frac{2 \hat{p}}{z_{A}} \sin \left(k R\left|\frac{z_{A}}{R-z_{A}}\right| \frac{\sin ^{2} \theta_{m}}{4}\right)
$$

When relating the pressure to the pressure in the focusing point:

$$
|f(A)| \approx\left|\frac{R}{R-z_{A}}\right| \frac{\sin \left(\frac{1}{2} k R\left(1-\cos \theta_{m}\right)\left|\frac{z_{A}}{R-z_{A}}\right|\right)}{\frac{1}{2} k R\left(1-\cos \theta_{m}\right)\left|\frac{z_{A}}{R-z_{A}}\right|}
$$

In the centre of the sphere: $z_{A}=0:|f(A)|=1$

Figure 7.6 shows a comparison between numerical results (7.1) and (7.35) for small and large $k R\left(1-\cos \theta_{m}\right.$ ) (from figure 7.2 situation a) and e)). The approximation by (7.35) gives a slightly higher result than the numerical result, but in general the differences are small. The difference may be explained from the taking into account the angle of incidence: In the numerical integration, the term $\cos \varphi$ (see (7.1)) is incorporated, but it is not in (7.35). Furthermore figure 7.6 shows that , for the smaller opening angle, the maximum is not at the focusing point but slightly closer to the reflector. 


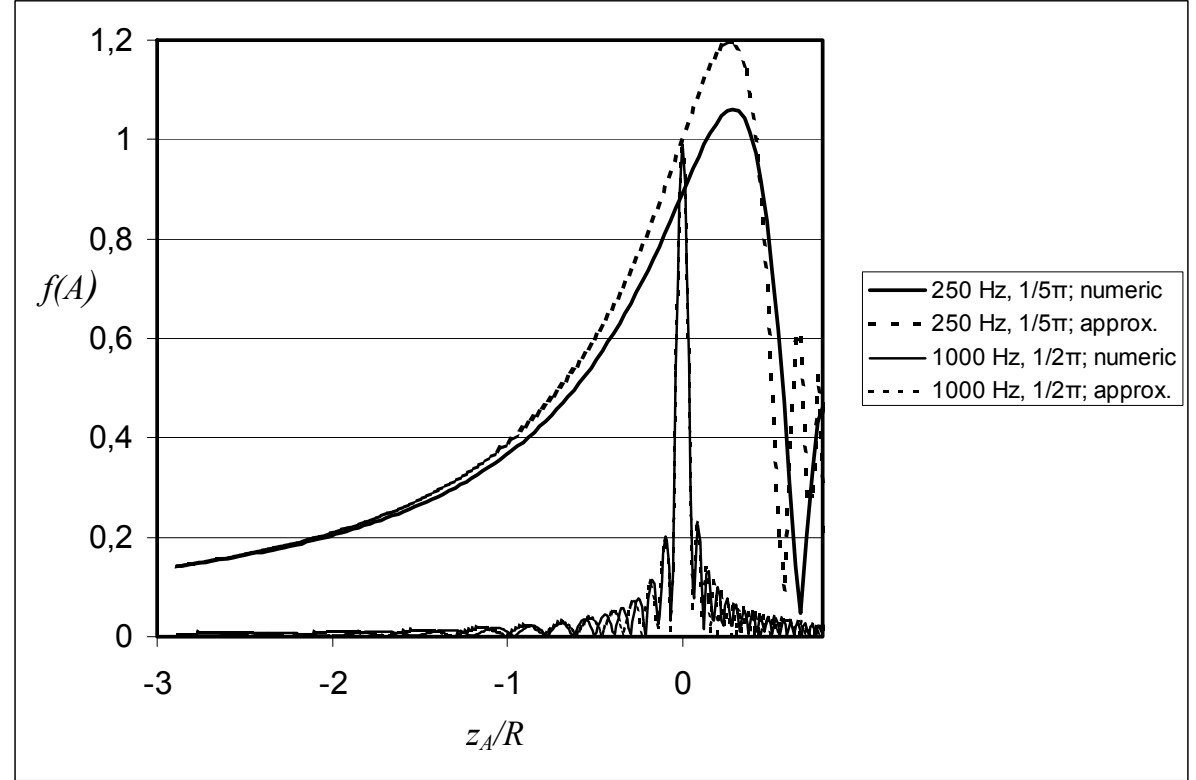

Figure 7.6. Sound pressure relative to the pressure in the centre along the axis $f(A)$. The $x$ axis is the relative distance $\left(z_{A} / R\right)$ to the centre. Results for 1000 and $250 \mathrm{~Hz}$ (see figure 7.2a) and e)), obtained by numeric calculation (7.1) and the approximation with (7.35).

The 'depth' (distance along the $z$-axis) of the focusing effect, described by the $-3 \mathrm{~dB}$ points will occur at $|f(A)|=\frac{1}{2} \sqrt{2}$. For $z_{A}<<R$ this can be approximated by:

$$
z_{A} \approx \pm 0,44 \frac{\lambda}{\left(1-\cos \theta_{m}\right)}
$$

Derivation: The $-3 \mathrm{~dB}$ points $|f(A)|=\frac{1}{2} \sqrt{2}$ will occur for:

$$
\left|\frac{R}{R-z_{A}}\right| \frac{\sin \left(\frac{1}{2} k R\left(1-\cos \theta_{m}\right)\left|\frac{z_{A}}{R-z_{A}}\right|\right)}{\frac{1}{2} k R\left(1-\cos \theta_{m}\right)\left|\frac{z_{A}}{R-z_{A}}\right|}=\frac{1}{2} \sqrt{2},
$$

for $z_{A}<<R$ :

$$
f(A) \approx \frac{\sin \left(\frac{1}{2} k\left(1-\cos \theta_{m}\right)\left|z_{A}\right|\right)}{\frac{1}{2} k\left(1-\cos \theta_{m}\right)\left|z_{A}\right|}=\frac{1}{2} \sqrt{2}
$$

This will be the case for:

$$
\frac{1}{2} k\left(1-\cos \theta_{m}\right)\left|z_{A}\right| \approx 1,3915
$$

which means that:

$$
\left|z_{A}\right| \approx \frac{1,3915 \lambda}{\pi\left(1-\cos \theta_{m}\right)} \approx 0,44 \frac{\lambda}{\left(1-\cos \theta_{m}\right)}
$$


For a further analysis of the decrease with distance, it is interesting to see in what situation there are no zeros beyond the focusing point. This will occur in (7.35) when:

$$
k R\left|\frac{z_{A}}{R-z_{A}}\right| \frac{\left(1-\cos \theta_{m}\right)}{2}<k R \frac{\left(1-\cos \theta_{m}\right)}{2}<\pi,
$$

so when

$$
\begin{aligned}
& k R\left(1-\cos \theta_{m}\right)<2 \pi, \text { or: } \\
& R\left(1-\cos \theta_{m}\right)<\lambda .
\end{aligned}
$$

It is noted that $R\left(1-\cos \theta_{m}\right)$ is the 'depth' of the sphere segment. The condition $R\left(1-\cos \theta_{m}\right)<\lambda$ means that less than one wavelength fits in this depth.

The following three situations can be distinguished:
A. $k R\left(1-\cos \theta_{m}\right)>2 \pi$ or $R\left(1-\cos \theta_{m}\right)>\lambda$
B. $\frac{1}{2} \pi<k R\left(1-\cos \theta_{m}\right)<2 \pi$ or $\lambda / 4<R\left(1-\cos \theta_{m}\right)<\lambda$
C. $k R\left(1-\cos \theta_{m}\right)<\frac{1}{2} \pi$ or $R\left(1-\cos \theta_{m}\right)<\lambda / 4$

Situation A (depth $R\left(1-\cos \theta_{m}\right)>\lambda$ ): Transition to geometrical decrease with distance

For situation $A$ the point will be considered where the curve will cross the curve of the geometric decrease with distance, assuming the centre point as the source (beyond that point strong interference's may be found, but these are of minor concern):

$$
|p(A, \omega)|=\frac{2 \hat{p}}{z_{A}} \sin \left(k R\left|\frac{z_{A}}{R-z_{A}}\right| \frac{\left(1-\cos \theta_{m}\right)}{2}\right)=\frac{\hat{p}}{z_{A}}
$$

This will be for $k R \frac{\left(1-\cos \theta_{m}\right)}{2} \frac{z_{A}}{R-z_{A}}=n \pi \pm \frac{1}{6} \pi$.

Particularly interesting will be the situation: $\pm \frac{5}{6} \pi$.

Since $k R\left(1-\cos \theta_{m}\right)$ is sufficiently large, the intersection points will be at:

$$
z_{A}= \pm \frac{5 R \lambda}{6 R\left(1-\cos \theta_{m}\right) \pm 5 \lambda} \approx \pm \frac{R \lambda}{R\left(1-\cos \theta_{m}\right) \pm \lambda}
$$

This is illustrated in figure 7.7. In between the intersection points (indicated with area $w$ ) the pressure can best be approximated using (7.35). Outside these points (indicated with $g$ ) the average pressure may be approximated by using a geometrical method. 


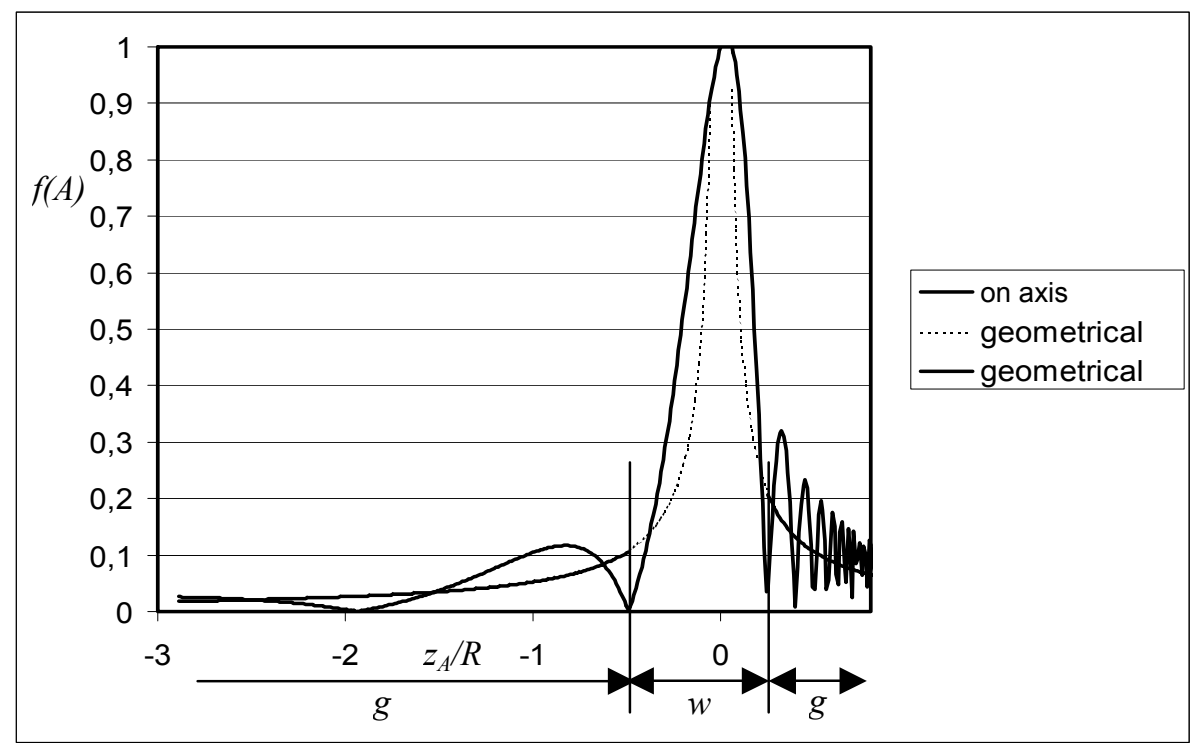

Figure 7.7. Calculated sound pressure relative to the pressure in the centre $f(A)$ as a function of the relative distance $z_{A} / R$ on $z$-axis. The decrease calculated with (7.35) is shown with a solid line and the geometrical decrease from the points indicated by (7.40) is shown here as a solid line and between these points it is shown as a dotted line. Calculation for situation of figure 7.2b: $k R\left(1-\cos \theta_{m}\right)=19$

\section{Situation $C$ (depth $R\left(1-\cos \theta_{m}\right)<\lambda / 4$ ): strong diffraction}

When the depth of the segment is less than a quarter wavelength, diffraction from the segment will occur, similar or even almost equal to the diffraction of a flat disk.

The pressure amplitude will be inversely proportional to the distance from the sphere instead of the distance from the centre:

$$
|p(z)|=\hat{p} \frac{k R\left(1-\cos \theta_{m}\right)}{R-z}
$$

where $R-z$ is the distance from the sphere along the $z$-axis.

If we assume a geometrical decrease with distance from the centre of the sphere segment (position: $R-z$ ), then $U$ should be a factor, independent of $z$ :

$$
|p(z)|=\frac{2 \hat{p}}{z_{A}} \sin \left(\frac{1}{2} k R\left|\frac{z}{R-z}\right|\left(1-\cos \theta_{m}\right)\right)=\left|\frac{U \hat{p}}{R-z}\right|
$$

For small $k R\left(1-\cos \theta_{m}\right)$ :

$$
\begin{aligned}
& \sin \left[\frac{z}{2(R-z)} k R\left(1-\cos \theta_{m}\right)\right] \approx \frac{z}{2(R-z)} k R\left(1-\cos \theta_{m}\right), \text { so: } \\
& \left|\frac{2 \hat{p}}{z_{A}}\left[\frac{z}{2(R-z)} k R\left(1-\cos \theta_{m}\right)\right]\right|=\left|\frac{\hat{p}}{(R-z)} k R\left(1-\cos \theta_{m}\right)\right|=\left|\frac{U \hat{p}}{R-z}\right|
\end{aligned}
$$

Meaning that:

$$
\begin{aligned}
& U=k R\left(1-\cos \theta_{m}\right) \text { and } \\
& p(z)=\hat{p} \frac{k R\left(1-\cos \theta_{m}\right)}{R-z}
\end{aligned}
$$


This is valid within $10 \%$ for $z<\frac{R \sqrt{2,4}}{\sqrt{2,4}+k R\left(1-\cos \theta_{m}\right)}$ (with $0<z<R$ )

The validity is limited to small arguments, for which $\sin x \approx x$.

The error can be approximated from the Taylor series:

$$
\sin x=x-\frac{x^{3}}{3 !}+\frac{x^{5}}{5 !}-\ldots+\ldots
$$

The error will be smaller than the 2 nd term, and the relative error will be: $\eta<\frac{x^{2}}{3 !}$.

If this error will be limited to $10 \%$ of the pressure:

$$
\begin{aligned}
& \eta=\frac{1}{6}\left(\frac{z}{2(R-z)}\right)^{2} k^{2} R^{2}\left(1-\cos \theta_{m}\right)^{2}<0,1, \text { or } \\
& \left|\frac{z}{(R-z)}\right|<\frac{\sqrt{2,4}}{k R\left(1-\cos \theta_{m}\right)}, \text { for } 0<z<R: \\
& \frac{z}{(R-z)}<\frac{\sqrt{2,4}}{k R\left(1-\cos \theta_{m}\right)}, \text { so } z<\frac{R \sqrt{2,4}}{\sqrt{2,4}+k R\left(1-\cos \theta_{m}\right)}
\end{aligned}
$$

Situation $B\left(\lambda / 4<R\left(1-\cos \theta_{m}\right)<\lambda\right)$ :

The situation for $\frac{1}{2} \pi<k R\left(1-\cos \theta_{m}\right)<2 \pi$ can be considered as an 'in between' situation with a virtual monopole somewhere between the sphere segment and the centre. A sort of beam will be obtained, as can be seen in figure 7.2 (situation c and e). Formula (7.35) has to be used to describe the decrease of the pressure, also at longer distances.

\section{Source position along the axis}

As a second step, the pressure will be considered if the source lies outside of the centre, along the axis of the sphere segment:

Parameters $Z$ and $Y$ according to $(7.18)$ will be:

$$
Z=0 \text { and } Y=k R \sin ^{2} \theta_{m}\left(\frac{z_{A}}{R-z_{A}}+\frac{z_{B}}{R-z_{B}}\right)
$$

From (7.22), this will result in:

$$
|p(A, \omega)|=\frac{2 \hat{p}}{\left(z_{A}+z_{B}-\frac{2}{R} z_{A} z_{B}\right)} \sin \left(\frac{1}{2} k R\left(1-\cos \theta_{m}\right)\left(\frac{z_{A}}{R-z_{A}}+\frac{z_{B}}{R-z_{B}}\right) \mid\right)
$$

If source and receiver are opposite the centre, according to $z_{A} / R-z_{A}=-z_{B} / R-z_{B}$, the pressure will be equal to the pressure calculated with the source in the centre. It has to be realized that due to spherical aberration the pressure in the focusing point for eccentric situations may be somewhat lower, as explained in chapter 7.4.2. 
The transition to the geometrical field can be determined by finding the first zero of (7.44). This will be for:

$$
\frac{z_{A}}{R-z_{A}}+\frac{z_{B}}{R-z_{B}}= \pm \frac{\lambda}{R\left(1-\cos \theta_{m}\right)}
$$

\section{Other source positions}

A third possibility will be that the source position is not in the centre but also not at the axis of the sphere segment.

The parameter $Z$, according to (7.18), will be zero along the specular line, with:

$$
\frac{x_{A}}{u^{\prime}}=-\frac{x_{B}}{s^{\prime}} .
$$

The parameter $Y$ according to (7.18) will be:

$$
Y=k R \sin ^{2} \theta_{m}\left(\frac{z_{A}}{u^{\prime}}+\frac{z_{B}}{s^{\prime}}\right)
$$

this will result in:

$$
|p(A, \omega)|=\frac{2 \hat{p} R}{\left(s^{\prime} z_{A}+u^{\prime} z_{B}\right)} \sin \left(\frac{1}{2} k R\left(1-\cos \theta_{m}\right)\left(\frac{z_{A}}{u^{\prime}}+\frac{z_{B}}{s^{\prime}}\right)\right)
$$

When the receiver positions are considered along the specular line, $z_{A}$ can be written as a function of $u$ :

$$
\begin{aligned}
& \cos \theta=\frac{R-z_{B}}{s^{\prime}}=\frac{R-z_{A}}{u^{\prime}} \quad \text {, so } \\
& z_{A}=R-u^{\prime} \cos \theta
\end{aligned}
$$

Now, from (7.49) to (7.51), the pressure can be written as a function of the distance $u$ ' from the reflector:

$$
|p(A, \omega)|=\frac{2 \hat{p} R}{R s^{\prime}+u^{\prime}\left(2 z_{B}-R\right)} \sin \left(\frac{1}{2} k R\left(1-\cos \theta_{m}\right)\left(\frac{R}{u^{\prime}}+\frac{2 z_{B}-R}{s^{\prime}}\right)\right)
$$

The pressure in $A$ related to the pressure in the focusing point will be:

$$
|f(A)|=\frac{R^{2}}{s^{\prime} u^{\prime}} \frac{\sin \left(\frac{1}{2} k R\left(1-\cos \theta_{m}\right)\left(\frac{R}{u^{\prime}}+\frac{2 z_{B}-R}{s^{\prime}}\right)\right)}{\frac{1}{2} k R\left(1-\cos \theta_{m}\right)\left(\frac{R}{u^{\prime}}+\frac{2 z_{B}-R}{s^{\prime}}\right)}
$$

It is noted that $(7.51)$ is equal to $(7.44)$ for normal incidence.

Beyond the point where interference starts to occur, the sound field may be approximated by the geometrical sound field. The first zeros will occur at:

$$
\frac{1}{u^{\prime}}=\frac{ \pm \lambda}{R^{2}\left(1-\cos \theta_{m}\right)}-\frac{2 z_{B}-R}{R s^{\prime}}
$$

It should be noted that the second zero will only occur when:

$$
\frac{2 z_{B}-R}{s^{\prime}}<-\frac{\lambda}{R\left(1-\cos \theta_{m}\right)}
$$




\subsubsection{Pressure in the focusing plane}

The pressure in the focusing plane of the sphere segment will be considered here, again both for the situation with the source at the centre of the sphere and in the situation with the source outside the centre.

\section{Source in the centre}

For the calculation of the pressure in the focusing plane, the source will be placed at the centre of the sphere and the pressure is considered at positions $\left(x_{A}, y_{A}, 0\right)$. Since this situation is cylindrically symmetrical, a variation over the $x$-axis will do and $y_{A}=0$ is assumed. For that situation, the $Y$ and $Z$ according to (7.18) will be:

$$
\begin{aligned}
& Y=k R \sin ^{2} \theta_{m}\left(\frac{z_{A}}{u^{\prime}}+\frac{z_{B}}{s^{\prime}}\right)=0 \\
& Z=k R \sin \theta_{m} \frac{x_{A}}{u^{\prime}}
\end{aligned}
$$

By applying formula (7.19), with $\lim (Y \rightarrow 0)$, only the first term of $u_{1}$ is needed:

$$
\begin{aligned}
& u_{1}(0, Z)=\frac{Y}{Z} J_{1}(Z) \text { and } \\
& u_{2}=0, \text { so: } \\
& I(Y, Z)=e^{-j \frac{Y}{2}} \frac{Y}{Z} J_{1}(Z)
\end{aligned}
$$

From (7.18) this will result in:

$$
|p(A, \omega)|=\hat{p} k \frac{R}{u^{\prime}} \sin ^{2} \theta_{m} \frac{J_{1}\left(k R \sin \theta_{m} \frac{x_{A}}{u^{\prime}}\right)}{k R \sin \theta_{m} \frac{x_{A}}{u^{\prime}}}
$$

The pressure in $A$ related to the pressure in the focusing point will be:

$$
\left|f\left(x_{A}\right)\right|=\frac{p(A, \omega)}{\hat{p} k\left(1-\cos \theta_{m}\right)}=\frac{R}{u^{\prime}} \frac{2 J_{1}\left(k R \sin \theta_{m} \frac{x_{A}}{u^{\prime}}\right)}{k R \sin \theta_{m} \frac{x_{A}}{u^{\prime}}}
$$

A comparison of (7.58) with numeric results from (7.1) is presented in figure 7.8.

For $k x_{A} \sin \theta_{m}<2$ (or $x_{A}<\lambda /\left(\pi \sin \theta_{m}\right)$ ) the function $f\left(x_{A}\right)$ can be simplified to:

$$
\left|f\left(x_{A}\right)\right| \approx \cos \left(\frac{1}{2} k R \sin \theta_{m} \frac{x_{A}}{u^{\prime}}\right) .
$$

This will only approximate the main lobe, as can be seen in figure 7.8 , but that will be sufficient for our purposes. 


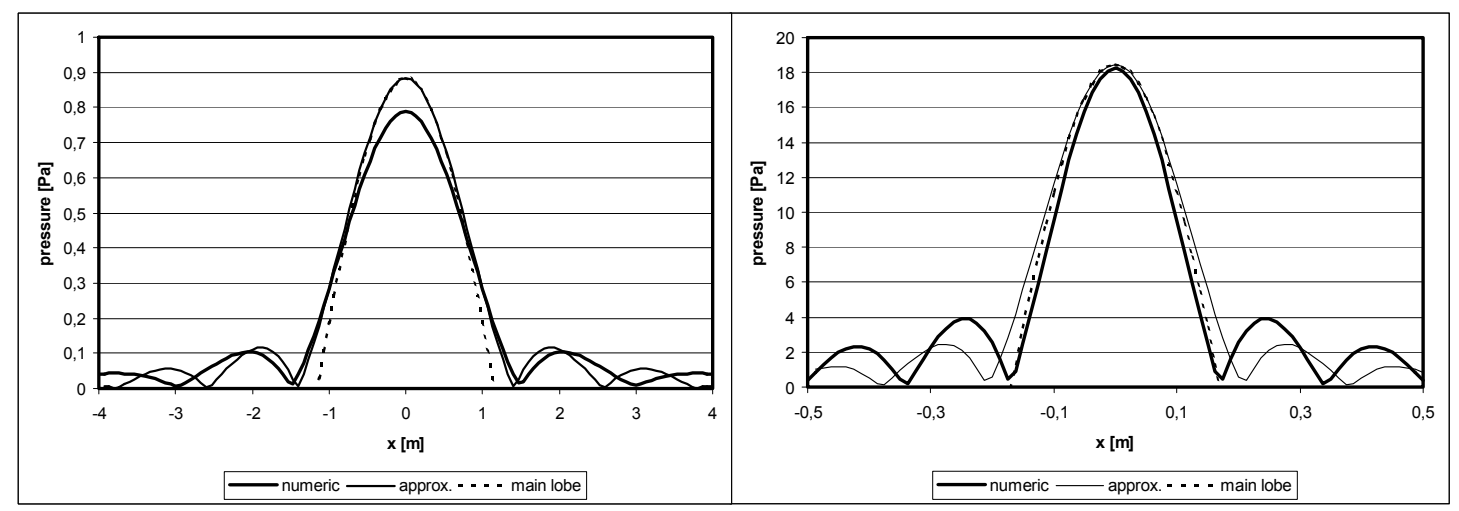

Figure 7.8. Pressure in the focusing plane. $R=5,4 \mathrm{~m}$. The source $(\hat{p}=1 \mathrm{~N} / \mathrm{m})$ is in the centre point. Left: $250 \mathrm{~Hz}, \theta_{m}=\frac{1}{5} \pi$ (fig.7.2 e), right: $1000 \mathrm{~Hz}, \theta_{m}=\frac{1}{2} \pi$ (fig.7.2 a),. Numerical calculation using (7.1), approximation using (7.58) and simplification with only the main lobe (7.60)

The width of the lobe, defined by the $-3 \mathrm{~dB}$ points, will be:

$$
-\frac{1}{4} \pi<\frac{1}{2} k R \sin \theta_{m} \frac{x_{A}}{u^{\prime}}<\frac{1}{4} \pi
$$

which is approximately $\left(u^{\prime} \approx R\right)$ :

$$
-\frac{\lambda}{4 \sin \theta_{m}}<x_{A}<\frac{\lambda}{4 \sin \theta_{m}}
$$

For a hemisphere $\left(\theta_{m}=\frac{1}{2} \pi\right)$ the total width of the lobe will be $\frac{1}{2} \lambda$.

Note: At the focusing $(x, y)$ plane $\left(z_{A}=0\right)$ of a hemisphere $\left(\theta_{m}=\frac{1}{2} \pi\right)$ the focusing area will be a circle with a diameter of half a wavelength:

$$
S_{F} \approx \frac{\pi}{16} \lambda^{2}
$$

The sound power $P_{S}$ of a sound source can be written as (5.34):

$$
P_{S}=\hat{p}^{2} \cdot 2 \pi / \rho c
$$

The power from a sound source in the centre, incident on a hemisphere, will be half this value:

$$
P_{I}=\hat{p}^{2} \cdot \pi / \rho c .
$$

This sound power will be reflected towards the focusing point. When averaging this reflected sound power over the focusing area $S_{F}$ the $r m s$ pressure will be:

$$
p_{r m s}{ }^{2}=\rho c P_{S} / S_{F} \approx 0,4 \hat{p}^{2} k^{2}
$$

this is close to the theoretical peak value (see paragraph 7.2):

$$
p_{r m s}^{2}=\frac{1}{2} \hat{p}^{2} k^{2}
$$

So, for a hemisphere, the energy is distributed over a circular area with a width of approximately half a wavelength. Outside this circular area, the receiver is either in the shadow zone (figure 5.7) and the SPL will decrease rapidly, or it is in the illuminated area (only for $\theta_{m} \geq \frac{1}{2} \pi$ ) and the average SPL (without interference) can be estimated with geometrical methods. 
Beyond the point where interference starts to occur, the sound field may be approximated by the geometrical sound field. To determine the distance from the intersection point of the geometrical field to the focusing point, the first zero of the $f\left(x_{A}\right)$ function can be used, approximately:

$$
x_{A}=\frac{\lambda}{2 \sin \theta_{m}}
$$

\section{Source outside the centre}

Next, the pressure in the focusing plane will be considered when moving the source along the $x$ axis $\left(y_{A}=y_{B}=0\right)$. The $Y$ and $Z$ according to (7.18) will be:

$$
Z=k R \sin \theta_{m}\left(\frac{x_{A}}{u^{\prime}}+\frac{x_{B}}{s^{\prime}}\right)
$$

A first approximation with $z_{A}=-z_{B} \approx 0$ results in $Y \approx 0$, so the $Y$ in (7.21) can be set to zero. From (7.22) the pressure will be:

$$
\begin{aligned}
|p(A, \omega)| & =\frac{\hat{p} R\left|e^{-j \frac{Y}{2}}\right|}{\left(s^{\prime} z_{A}+u^{\prime} z_{B}\right)}\left|\frac{Y}{Z} J_{1}(Z)\right| \cdot \\
& =\frac{\hat{p} R}{\left(s^{\prime} z_{A}+u^{\prime} z_{B}\right)}\left|\frac{k R \sin ^{2} \theta_{m}\left(\frac{z_{A}}{u^{\prime}}+\frac{z_{B}}{s^{\prime}}\right)}{k R \sin \theta_{m}\left(\frac{x_{A}}{u^{\prime}}+\frac{x_{B}}{s^{\prime}}\right)} J_{1}\left(k R \sin \theta_{m}\left(\frac{x_{A}}{u^{\prime}}+\frac{x_{B}}{s^{\prime}}\right)\right)\right|
\end{aligned}
$$

or, when relating to the pressure in the focusing point:

$$
|f(x)|=\frac{R^{2}}{s^{\prime} u^{\prime}}\left|\frac{2 J_{1}\left(k R \sin \theta_{m}\left(\frac{x_{A}}{u^{\prime}}+\frac{x_{B}}{s^{\prime}}\right)\right)}{k R \sin \theta_{m}\left(\frac{x_{A}}{u^{\prime}}+\frac{x_{B}}{s^{\prime}}\right)}\right|
$$

For the main lobe $\left(\frac{x_{A}}{u^{\prime}}+\frac{x_{B}}{s^{\prime}}\right)<\frac{\lambda}{2 R \sin \theta_{m}}$, the function $f$ can be simplified as:

$$
\left|f\left(x_{A}\right)\right| \approx \cos \left(\frac{1}{2} k R \sin \theta_{m}\left(\frac{x_{A}}{u^{\prime}}+\frac{x_{B}}{s^{\prime}}\right)\right) .
$$

Since for the focusing point $x_{B} / s^{\prime}=-x_{A} / u^{\prime}=\sin \theta$, this means that (approximately) the pressure field will 'move' towards the direction of the specular reflection.

With point $A$ being the focusing point, $x_{r}$ the distance relative to $x_{A}$, and considering specular reflection $\left(x_{B} / s^{\prime}=-x_{A} / u^{\prime}\right),(7.66)$ will become:

$$
\left|f_{f p}\left(x_{A}\right)\right| \approx \cos \left(\frac{1}{2} k R \sin \theta_{m}\left(\frac{x_{r}}{u^{\prime}}\right)\right)
$$


It is noted however, that this will only be valid only for small deviations from the centre, since the effects described in par 7.4.2 and figure 7.5 have to be taken into account.

If the source is not in the plane through the centre, $z_{B} \neq 0$, the assumption of $Y \approx 0$ in the focusing plane may not be valid anymore and the approximation becomes less accurate. 


\subsubsection{Pressure in the far field}

The pressure in the far field will be considered here, both for the situation with the source in the centre of the sphere segment and in the situation with the source outside the centre.

\section{Source in the centre}

The source will be placed in the centre of the sphere again and the pressure in the far field will be considered. This situation is cylindrical symmetrical, so $y_{A}=0$ can be assumed, and $\frac{x_{A}}{u^{\prime}}=\sin \theta_{r}$. Since $z_{A}>>$ it can be assumed that:

$$
\frac{z_{A}}{u^{\prime}} \approx \frac{z_{A}-R}{u^{\prime}}=\cos \theta_{r} .
$$

This will result in parameters $Y$ and $Z$ according to (7.18):

$$
\begin{aligned}
Y & =k R \sin ^{2} \theta_{m}\left(\frac{z_{A}}{u^{\prime}}+\frac{z_{B}}{s^{\prime}}\right) \\
& \approx k R \sin ^{2} \theta_{m} \cos \theta_{r} \quad, \text { and } \\
Z & =k R \sin \theta_{m} \sqrt{\left(\frac{x_{A}}{u^{\prime}}+\frac{x_{B}}{s^{\prime}}\right)^{2}+\left(\frac{y_{B}}{s^{\prime}}+\frac{y_{A}}{u^{\prime}}\right)^{2}} \\
& =k R \sin \theta_{m} \sin \theta_{r} \\
\text { So } \frac{Y}{Z} & =\frac{\sin \theta_{m}}{\tan \theta_{r}} \text { and } \frac{Y}{2}+\frac{Z^{2}}{2 Y}=\frac{1}{2} k R\left(\sin ^{2} \theta_{m} \cos \theta_{r}+\frac{\sin ^{2} \theta_{r}}{\cos \theta_{r}}\right)
\end{aligned}
$$

When the pressure near the axis of the sphere segment $(Z<<Y)$ is considered, $\cos \theta_{r} \approx 1$ can be assumed, which results in:

$$
\frac{Y}{2}+\frac{Z^{2}}{2 Y} \approx \frac{1}{2} k R\left(\sin ^{2} \theta_{m}+\sin ^{2} \theta_{r}\right)
$$

In the illuminated zone, for $Z<Y$, so $\tan \theta_{r}<\sin \theta_{m}$, (7.22) can be used, giving:

$$
|I(Y, Z)|=\left\{\begin{array}{l}
\left(J_{o}\left(k R \sin \theta_{m} \sin \theta_{r}\right)-\cos \left(\frac{1}{2} k R\left(\sin ^{2} \theta_{m}+\sin ^{2} \theta_{r}\right)\right)\right)^{2}+ \\
\left(\frac{\tan \theta_{r}}{\sin \theta_{m}} J_{1}\left(k R \sin \theta_{m} \sin \theta_{r}\right)-\sin \left(\frac{1}{2} k R\left(\sin ^{2} \theta_{m}+\sin ^{2} \theta_{r}\right)\right)\right)^{2}
\end{array}\right\}^{\frac{1}{2}}
$$

This expression is only sufficiently accurate for small $\theta_{m}$ and small $\theta_{r}$.

With the source in the centre (7.22) will reduce to:

$$
|p(A, \omega)|=\frac{\hat{p}}{z_{A}}|I(Y, Z)|
$$

Formulas (7.72) and (7.73) describe the interference pattern in the far field. However, the deviations around the geometrical value are limited, so for most room acoustical purposes the geometrical approximation will be sufficient. 
There are however some interesting cases to consider:

When $k R\left(1-\cos \theta_{m}\right)=\pi, 3 \pi, . ., n \pi$ with $n$ being an uneven whole number (the 'depth' of the sphere segment corresponds to $\left.\frac{1}{2} n \lambda\right)$, there is a pronounced maximum on the axis $\left(\theta_{r}=0\right)$. For small angles $\theta_{r},|I(Y, Z)|$ can be approximated by:

$$
\begin{aligned}
& |I(Y, Z)| \approx\left\{1+J_{o}\left(k R \sin \theta_{m} \sin \theta_{r}\right)^{2}-2 J_{o}\left(k R \sin \theta_{m} \sin \theta_{r}\right) \cos \left(\frac{1}{2} k R\left(\sin ^{2} \theta_{m}+\sin ^{2} \theta_{r}\right)\right)\right\}^{\frac{1}{2}}(7.72) \\
& |I(Y, Z)| \approx 1+\cos \left(\frac{1}{\sqrt{2}} k R \sin \theta_{m} \sin \theta_{r}\right)
\end{aligned}
$$

On the axis $\left(\theta_{r}=0\right)$ this will result in $|I(Y, Z)|=2$. The pressure will be twice the pressure based on the geometrical approach.

Formula (7.75) can be applied for $\cos \left(\frac{1}{\sqrt{2}} k R \sin \theta_{m} \sin \theta_{r}\right)>0$, so:

$$
\begin{aligned}
& \left|\frac{1}{\sqrt{2}} k R \sin \theta_{m} \sin \theta_{r}\right|<\frac{1}{2} \pi, \text { therefore } \\
& \left|\sin \theta_{r}\right|<\frac{\sqrt{2}}{4} \frac{\lambda}{R \sin \theta_{m}}
\end{aligned}
$$

When $k R\left(1-\cos \theta_{m}\right)=2 \pi, 4 \pi, . ., n \pi$ with $n$ being an even whole number (the 'depth' of the sphere segment corresponds to $\left.\frac{1}{2} n \lambda\right)$, there is a minimum on the axis $\left(\theta_{r}=0\right)$.

Some calculation examples are given in figure 7.9

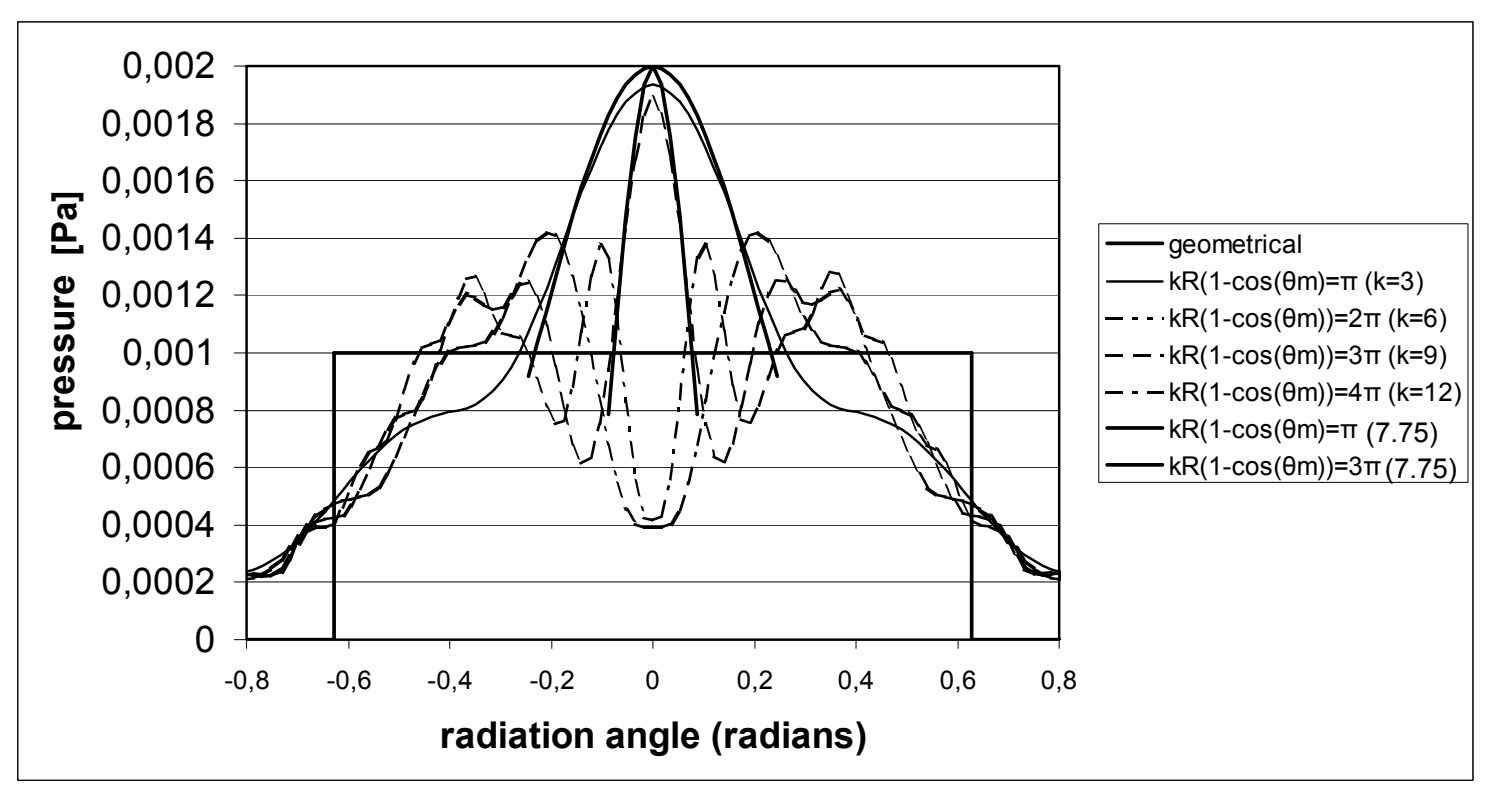

Figure 7.9. Calculated pressure, using (7.1), in the far field $(1000 \mathrm{~m})$ as a function of the radiation angle. Source $(\hat{p}=1 \mathrm{~N} / \mathrm{m})$ is in the centre. Calculations for different frequencies, corresponding with 'depth' $=\frac{1}{2} n \lambda . R=5,4 \mathrm{~m}, \theta_{m}=\frac{1}{5} \pi$. Also showing the approximated peaks using (7.75). The rectangle indicates the geometrical solution. 
The pressure at the borders of the illuminated area (in the case of figure 7.9 indicated by the geometrical approach: $\pm \theta_{m}= \pm \frac{1}{5} \pi \approx \pm 0,62$ ) is roughly half the geometrical pressure.

For a shallow sphere segment, $k R\left(1-\cos \theta_{m}\right) \leq \frac{1}{2} \pi$ a diverging sound field will be obtained, outside the borders of the 'illuminated' area.

For this small depth the pressure on the axis $\left(\sin \theta_{r}=0\right)$ will be:

$$
|p(A, \omega)| \approx \frac{\hat{p}}{z_{A}} k R \sin ^{2} \theta_{m}
$$

Defining the 'radius' $a=R \sin \theta_{m}$ and the area $S=\pi a^{2}$ of the sphere segment, the pressure can be written:

$$
|p(A, \omega)|=\hat{p} \frac{2 S}{\lambda R z_{A}}
$$

This is equal to the well-known Fraunhofer diffraction (see par. 7.3).

This situation will also occur in the limiting case with $a=R \sin \theta_{m}$ and $R \rightarrow \infty$.

Depending on the radiation angle $\theta_{r}$ the pressure can be approximated by:

$$
|p(A, \omega)|=\hat{p} \frac{k a^{2}}{R u^{\prime}} \frac{\sin \left(k a \sin \theta_{r}\right)}{k a \sin \theta_{r}}
$$

The approximation used is:

$$
\frac{2 J_{1}(x)}{x} \approx \frac{\sin (x)}{x}
$$

This is valid for $|x| \leq \pi$, so

$$
\left|k a \sin \theta_{r}\right|<\pi \text {, or }\left|\sin \theta_{r}\right|<\frac{\lambda}{2 R \sin \theta_{m}}
$$

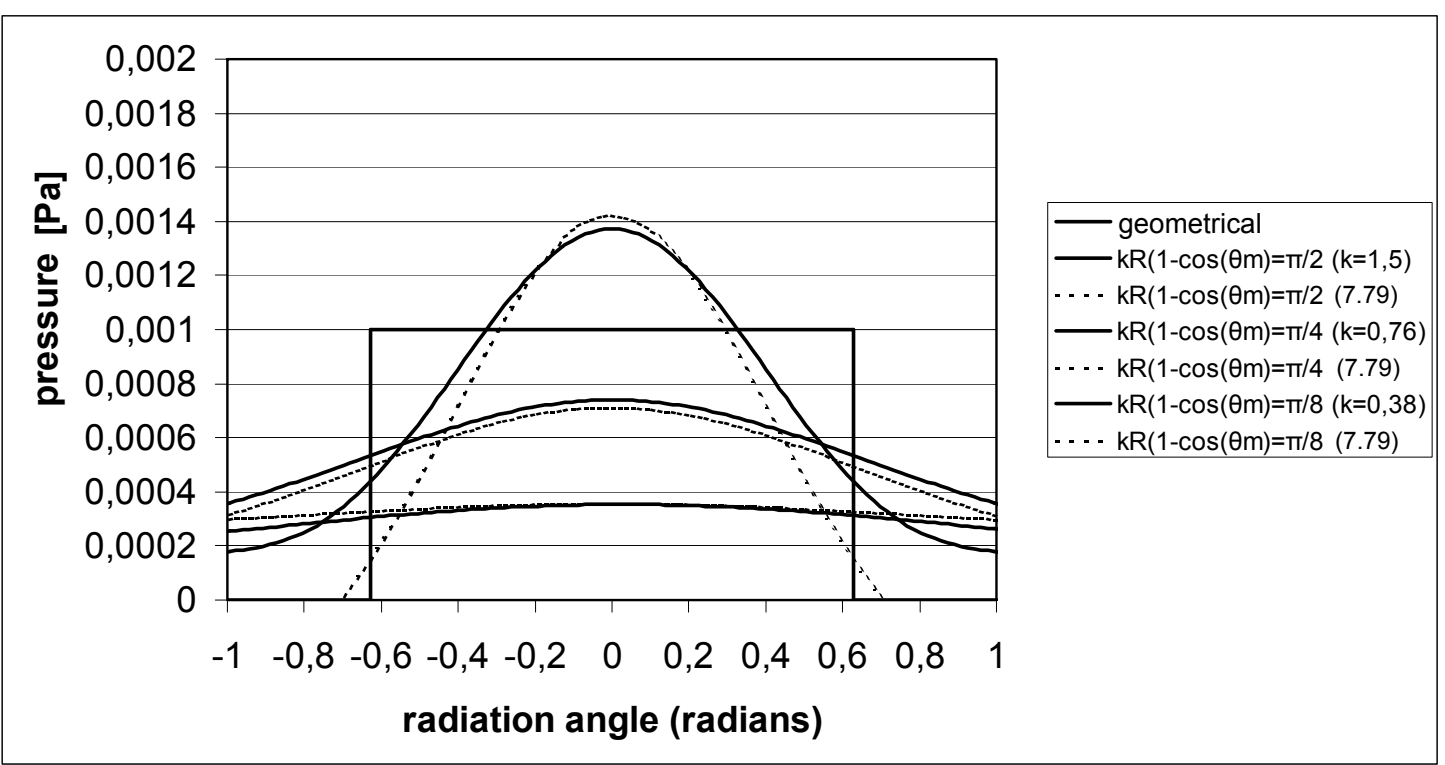

Figure 7.10. Calculated pressure for a sphere segment with small depth, using (7.1)(solid lines), in the far field $(1000 \mathrm{~m})$. Source $(\hat{p}=1 \mathrm{~N} / \mathrm{m})$ is in the centre Calculations for different frequencies, corresponding with 'depth' $=\frac{1}{4} \lambda, \frac{1}{8} \lambda, \frac{1}{16} \lambda . R=5,4 m, \theta_{m}=\frac{1}{5} \pi$. 


\section{Source outside the centre}

First, the source will be positioned outside the centre of the sphere on the $z$-axis $\left(x_{B}=y_{B}=0\right)$ and the pressure in the far field will be considered.

This will be a symmetrical situation, so $y_{A}=0$ can be assumed.

This will result in parameters $Y$ and $Z$ according to (7.18):

$$
Y=k R \sin ^{2} \theta_{m}\left(\cos \theta_{r}+\frac{z_{B}}{s^{\prime}}\right)
$$

And $Z$ will remain:

$$
Z=k R \sin \theta_{m} \sin \theta_{r}
$$

With (7.18) this will result in:

$$
\frac{Y}{2}+\frac{Z^{2}}{2 Y}=\frac{1}{2} k R\left(\sin ^{2} \theta_{m}\left(\cos \theta_{r}+\frac{z_{B}}{s^{\prime}}\right)+\frac{\sin ^{2} \theta_{r}}{\left(\cos \theta_{r}+\frac{z_{B}}{s^{\prime}}\right)}\right)
$$

For $x_{A}=\sin \theta_{r}=0$ (on the $z$-axis), the $Z=0$ and:

$$
\frac{Y}{2}+\frac{Z^{2}}{2 Y}=\frac{1}{2} k R \sin ^{2} \theta_{m}\left(1+\frac{z_{B}}{s^{\prime}}\right)
$$

From (7.22) it can be obtained:

$$
|I(Y, Z)| \approx \sqrt{2-2 \cos \left(\frac{1}{2} k R \sin ^{2} \theta_{m}\left(1+\frac{z_{B}}{s^{\prime}}\right)\right)}
$$

and the pressure can be determined:

$$
|p(A, \omega)|=\frac{\hat{p} R}{s^{\prime} u^{\prime}\left(1+\frac{z_{B}}{s^{\prime}}\right)} \sqrt{2-2 \cos \left(\frac{1}{2} k R \sin ^{2} \theta_{m}\left(1+\frac{z_{B}}{s^{\prime}}\right)\right)}
$$

The first part of (7.85) describes the geometrical effect, the root describes the interference (between 0 and 2), similar to the situation with the source in the centre. The maximum will occur at a different 'depth' of the sphere segment.

Secondly, the source will be moved along the $x$-axis $\left(z_{B}=y_{B}=0\right)$ and the pressure in the far field will be considered:

Assuming $y_{A}=0, \frac{x_{A}}{u^{\prime}}=\sin \theta_{r}$ and $\frac{x_{B}}{s^{\prime}}=\sin \theta_{s}$, this will result in parameters $Y$ and $Z$ according to (7.18):

$$
\begin{aligned}
& Y=k R \sin ^{2} \theta_{m} \cos \theta_{r} \\
& Z=k R \sin \theta_{m}\left(\sin \theta_{r}+\sin \theta_{s}\right)
\end{aligned}
$$

At the direction of the specular reflection, for $\theta_{r}=-\theta_{s}, Z=0$ and the result will be similar to the $z$-axis with the source in the centre. The pressure field is rotated over an angle $-\theta_{r}$. 


\section{$\underline{7.5 \text { Diffuse reflections in a sphere }}$}

In the previous sections a full reflection is assumed against a hard surface, so there will be a clear phase relation of the contributing signals in the reception point. In the case of perfect diffuse reflections (momentarily disregarding the practical possibilities of achieving this), there is random phase relation at each reception point, so energy will be added instead of pressure.

With the source in the centre of a hemisphere, the incident sound intensity $I_{i}$ on the surface element $d S$ of the hemisphere with radius $R$ will be:

$$
I_{i}=\frac{\hat{p}^{2}}{2 \rho c \cdot R^{2}}
$$

Thus, the incident power on a small surface element $d S$ of the hemisphere will be:

$$
d P_{i}=I_{i} d S=\frac{\hat{p}^{2} d S}{2 \rho c \cdot R^{2}}
$$

All the surfaces $d S$ will act as independent radiators. For each of these radiators a Lambert radiation directionality will be assumed. The intensity of Lambert radiation is dependent on the angle $\theta$ with the normal to the radiating surface: $I_{\varphi}=I_{0} \cdot \cos \theta$. The total power $P$ that is radiated by the Lambert radiator will result from integrating over the radiating surfaces, as seen from the radiator:

$$
P=I_{0} u^{2} \int_{0}^{2 \pi} \int_{0}^{\frac{1}{2} \pi} \sin \theta \cos \theta d \theta d \phi=\pi u^{2} I_{0}
$$

where $I_{0}$ is the on-axis sound intensity at distance $u$ from the radiating surface.

This means that the angle-dependent intensity of each Lambert radiator will be:

$$
I_{\varphi}=\frac{P}{\pi u^{2}} \cdot \cos \theta
$$

Assuming that all incident energy will be reflected, the incident power $d P_{i}$ of (7.89) can be entered in (7.91) for the radiant power $P$, obtaining the intensity in a point at angle $\theta$ and distance $u$ from surface element $d S$ :

$$
d I_{\varphi}=\frac{\hat{p}^{2} d S}{2 \pi \rho c u^{2} R^{2}} \cos \theta
$$

The total pressure at distance $u$ results from integration over $d S$ :

$$
p_{r m s}^{2}=\rho c \cdot I=\int_{s} \frac{\hat{p}^{2}}{2 \pi} \frac{\cos \theta}{u^{2} R^{2}} d S
$$

In the centre of the hemisphere this reduces to $(u=R, \cos \theta=1)$ :

$$
p_{r m s}^{2}=\frac{\hat{p}^{2}}{2 \pi R^{4}} \int_{s} d S=\frac{\hat{p}^{2}}{R^{2}}
$$

Therefore, the rms pressure is inversely proportional to the square of the radius.

The pressure of the specular reflecting concave reflector $(7.3 \mathrm{c})$ is much higher than the pressure from the perfect diffuse reflector (7.94). 
Solving the problem of the focusing effect could therefore theoretically be done by generating diffuse reflections. The practical possibilities will be discussed in chapter 13.1

\section{$\underline{7.6 \text { Conclusions on the sound field in a sphere }}$}

Based on the general Kirchhoff Integral (6.18), in this chapter the integral is formulated for the reflected sound field from a spherical reflector, in polar coordinates.

For the specific situation with the source and the receiver in the centre of the spherical reflector, a closed form solution is found.

For all other situations, approximations of the integral solution have to be made to describe the sound field.

With the approximations made, the sound pressure along the specular reflection axis, in the focusing plane and in the far field is described. Further, the influence of a sound source outside the centre of the spherical reflector is investigated for each of these situations.

The approximations are compared to numerical solutions using (7.1). For all described situations sufficiently accurate approximations are obtained.

In the next chapters the sound field in other curved shapes will be investigated. 


\section{Chapter 8 \\ The sound field in a cylinder}

In this chapter the general formulation of the Kirchhoff integral (6.18) is applied to the specific situation of reflections from a cylinder. An important difference between the sphere and the cylinder is that in a sphere the reflections are converging in two directions, while in a cylinder the reflections will converge in one direction and will diverge in the other.

Approximate solutions are given in the literature ([Kuttruff92], [Yamada05]) and will be summarized here for completeness.

Additionally, the theory is extended for sources outside the axis of the cylinder.

\subsection{Integral formulation for a cylinder}

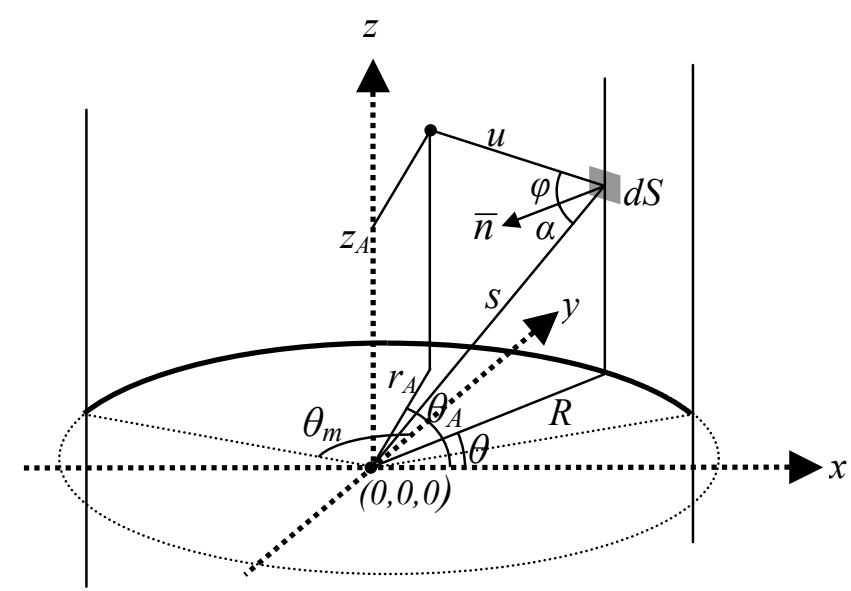

Figure 8.1. Cylindrical coordinates

The geometry of a circular cylindrical reflector is shown in figure 8.1. The origin is on the cylindrical axis. The positions within the cylinder are described by cylindrical coordinates $(r, \theta, z)$. The surface elements on the cylinder have dimension $d S=R d \theta d z$. The source is assumed in the origin $(0,0,0)$ and the pressure on the cylinder's surface will be:

$$
p(s, \omega)=\hat{p} \frac{e^{-j k s}}{s}
$$

where: $s=\sqrt{R^{2}+z^{2}}$ and $R=$ radius of the cylinder.

For receiver point $A$ the distance to the radiating element $d S$ will be $u$. The Kirchhoff integral (6.2) can then be written:

$$
\begin{aligned}
& p(A, \omega)=\frac{\hat{p}}{4 \pi} \int_{S}\left(\frac{1+j k s}{s} \cos \alpha+\frac{1+j k u}{u} \cos \varphi\right) \frac{e^{-j k(u+s)}}{s u} d S \\
& =\frac{\hat{p} R}{4 \pi} \int_{-\infty-\theta_{m}}^{\infty} \int_{\theta_{m}}^{\theta_{m}}\left(\frac{1+j k s}{s} \cos \alpha+\frac{1+j k u}{u} \cos \varphi\right) \frac{e^{-j k(u+s)}}{s u} d \theta d z
\end{aligned}
$$

Assuming source and receiver in the far field $(s>>\lambda, u>>\lambda)$ :

$$
p(A, \omega)=\frac{j \hat{p}}{\lambda} \int_{S}\left(\frac{\cos \alpha+\cos \varphi}{2}\right) \frac{e^{-j k(u+s)}}{s u} d S
$$




\subsection{Pressure at the origin of a cylinder}

\subsubsection{Pressure at the origin of a circular cylinder}

First the pressure in the origin will be determined, with the source also in the origin.

Derivation: For a sound source and the receiver positioned in $(0,0,0),(8.2)$ can be simplified by using $u=s$ and $\cos \alpha=\cos \varphi=\frac{R}{u}$. For a full circular cylinder $\left(\theta_{m}=\pi\right)$ :

$$
p(O, \omega)=\frac{\hat{p} R}{4 \pi} \int_{-\infty-\pi}^{\infty} \int_{u}^{\pi}\left(\frac{1+j k u}{u} \frac{R}{u}+\frac{1+j k u}{u} \frac{R}{u}\right) \frac{e^{-j k 2 u}}{u^{2}} d \theta d z
$$

Assuming a far field situation $(u>>\lambda)$, the near field components can be neglected. Furthermore, with source and receiver in $(0,0,0)$, the distance $u$ is independent of $\theta$. This will result in:

$$
p(O, \omega)=j \frac{\hat{p} R^{2}}{\lambda} \int_{-\infty-\pi}^{\infty} \int^{\pi} \frac{e^{-j k 2 u}}{u^{3}} d \theta d z=j \hat{p} k R^{2} \int_{-\infty}^{\infty} \frac{e^{-j k 2 u}}{u^{3}} d z
$$

Since $u=\sqrt{R^{2}+z^{2}}$ :

$$
p(O, \omega)=j \hat{p} k R^{2} \int_{-\infty}^{\infty} \frac{e^{-2 j k \sqrt{R^{2}+z^{2}}}}{\left(R^{2}+z^{2}\right)^{3 / 2}} d z
$$

For an infinite circular cylinder, (8.2) can be approximated by the single integral:

$$
p(O, \omega)=j \hat{p} k R^{2} \int_{-\infty}^{\infty} \frac{e^{-2 j k \sqrt{R^{2}+z^{2}}}}{\left(R^{2}+z^{2}\right)^{3 / 2}} d z
$$

and for a finite, open cylinder from $-z_{m}$ to $z_{m}$ :

$$
p(O, \omega)=j \hat{p} k R^{2} \int_{-z_{m}}^{z_{m}} \frac{e^{-2 j k \sqrt{R^{2}+z^{2}}}}{\left(R^{2}+z^{2}\right)^{3 / 2}} d z
$$

The integral part of this expression is a highly oscillating function related to the Fresnel integrals. Figure 8.2 shows a calculation example of (8.7) for integration between $-z_{m}$ and $z_{m}$.

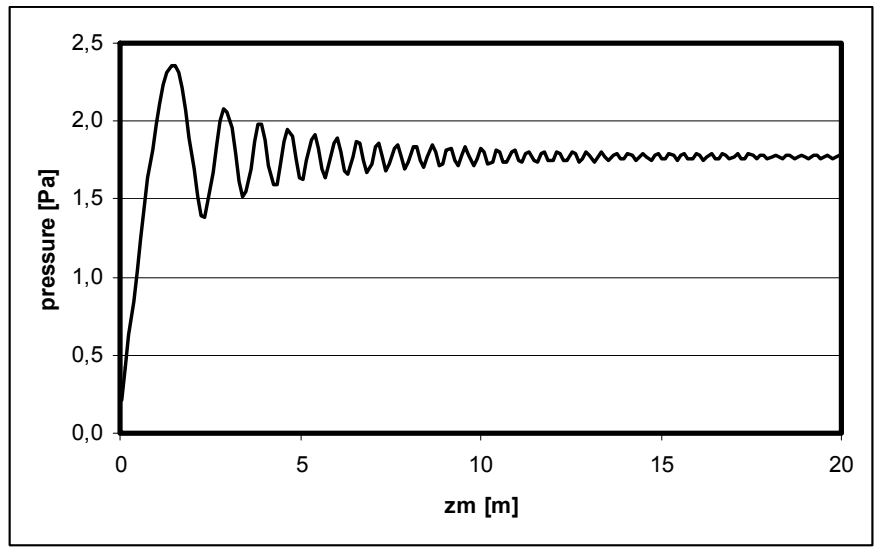

Figure 8.2. Calculation example of (8.7) for increasing the integration limit $z_{m} ; \hat{p}=1 \mathrm{~N} / \mathrm{m}$, $k=10, R=10 \mathrm{~m}$. 
The first maximum is reached when the size of the cylinder has the dimension of the first Fresnel zone: $z_{m}=\sqrt{\frac{1}{2} \lambda R}$.

The integral is solved with the stationary phase method in [Kuttruff92] and [Yamada05]: (for $R>\lambda$ ):

$$
p(O, \omega)=\hat{p}(1+j) \sqrt{\frac{\pi k}{2 R}} e^{-2 j k R}
$$

For a cylindrical segment, integration of (8.5) takes place over the opening angle from $-\theta_{m}$ to $\theta_{m}$. The pressure in the origin $(0,0,0)$ will be :

$$
p(O, \omega)=j \hat{p} k R^{2} \frac{\theta_{m}}{\pi} \int_{-\infty}^{\infty} \frac{e^{-j k 2 u}}{u^{3}} d z=\hat{p}(1+j) \frac{\theta_{m}}{\pi} \sqrt{\frac{\pi k}{2 R}} e^{-2 j k R}
$$

The amplitude of the pressure will be:

$$
|p(O, \omega)|=\hat{p} \frac{\theta_{m}}{\pi} \sqrt{\frac{\pi k}{R}}
$$

The rms value of the pressure will be:

$$
p_{r m s}^{2}=\frac{1}{2} \hat{p}^{2}\left(\frac{\theta_{m}}{\pi}\right)^{2} \frac{\pi k}{R}
$$

This is valid for sufficiently large $z_{m}$. For small $z_{m}$ the accuracy of this expression is within 2-3 dB (see figure 8.2), provided that $z_{m}>\sqrt{\frac{1}{2} \lambda R}$.

With these results the maximum sound pressure in the focal point, due to reflections from circular cylindrical curved surfaces, can be calculated. As with the sphere, the result is strongly dependent on the frequency. High frequencies give strong amplification in the focal point. Contrary to the sphere, the pressure in the focal point depends on the radius of curvature. The sound wave is concentrated in one direction but is still able to diverge in the other direction.

A comparison between the amplification in a (hemi)sphere and a cylinder is presented in chapter 12 . 


\subsubsection{Pressure at the focal point of an elliptical cylinder}

In [Yamada05] the pressure in the focal point is also given for an elliptical cylinder with the source in one of the focal axes and the receiver in the other, at the same height. For a full elliptical cylinder $\left(\frac{x^{2}}{a^{2}}+\frac{y^{2}}{b^{2}}=1, a>b\right)$ this is:

$$
\begin{aligned}
& p=\hat{p}(1-j) \frac{E I}{2 \pi} \sqrt{\frac{\pi k}{2 a}} \cdot e^{j 2 k a}, \quad \text { with } \\
& E I=\int_{0}^{2 \pi} \frac{1}{\sqrt{1-\left(1-\frac{a^{2}}{b^{2}}\right) \sin ^{2} \xi}} d \xi
\end{aligned}
$$

The elliptical integral $E I$ in (8.10) expresses a slight reduction of the pressure in the focal point in case the ellipse is oblong (see figure 8.3). The elliptical integral can be approximated for $1<a / b<2$ :

$$
E I \approx 2 \pi \sqrt{\frac{b}{a}}
$$

The result is almost equal to the circular cylinder, except for the slight reduction shown in figure 8.3 .

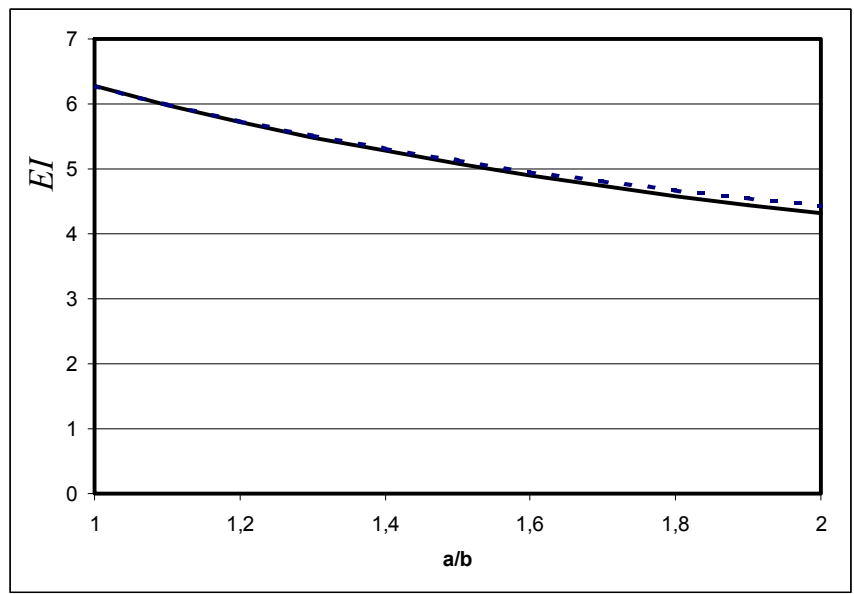

Figure 8.3. Elliptical integral EI in (8.10) as a function of a/b, solid line. The dotted line shows the approximation using (8.11).

Note: For a partial elliptical cylinder [Yamada05]:

$$
E I=\int_{0}^{\varphi_{2}} \frac{1}{\sqrt{1-\left(1-\frac{a^{2}}{b^{2}}\right) \sin ^{2} \xi}} d \xi-\int_{0}^{\varphi_{1}} \frac{1}{\sqrt{1-\left(1-\frac{a^{2}}{b^{2}}\right) \sin ^{2} \xi}} d \xi
$$

where

$$
\varphi=\sin ^{-1}\left[\frac{b_{e} \sin \phi d d_{e}^{2} \cos \phi+a e_{e}\left(a_{e}^{2}+d_{e}^{2} \sin ^{2} \phi\right)^{1 / 2}}{a_{e}}\right] .
$$

and $d_{e}=\sqrt{a_{e}^{2}-b_{e}^{2}}$ 


\subsection{Approximate solution of the sound field in a circular cylinder}

For source and/or receiver positions outside the centre axis of the cylinder, there is no closed form solution for (8.2) available. Approximations, made by taking the first terms from the Taylor series of the root, are necessary to be able to describe the sound field.

\subsubsection{Approximate solution of the integral for a cylinder}

An approximate solution for the sound field in a circular cylinder with the source in the origin will be given, according to the derivation in [Kuttruff92].

The following approximations will be made:

- $\quad$ A far field situation will be assumed

- $\quad$ The receiver point $A$ will be assumed to be relatively close to the source. It will be assumed that $\cos \varphi=\cos \alpha$

Derivation: The geometry of the problem is indicated in figure 8.1.

Using $\cos \alpha=\cos \varphi=\frac{R}{s}$, (8.2) can be written:

$$
p(A, \omega)=j k \frac{\hat{p} R^{2}}{2 \pi} \int_{-\infty}^{\infty} \int_{0}^{2 \pi} \frac{e^{-j k(u+s)}}{s^{2} u} d \theta d z
$$

From the geometry (see figure 8.1) the distance $u$ from the surface $d S$ on the cylinder to receiver point $A\left(r_{A}, \theta_{A}, z_{A}\right)$ can be described as:

$$
u^{2}=R^{2}+r_{A}^{2}-2 R r_{A} \cos \theta_{A}+\left(z_{A}-z\right)^{2}
$$

For small $r_{A}$ this can be approximated by:

$$
u^{2}=R^{2}+\left(z_{A}-z\right)^{2}-2 R r_{A} \cos \theta_{A}
$$

The first two terms of the Taylor series will be used for $u$ in the phase:

$$
u=\sqrt{R^{2}+\left(z_{A}-z\right)^{2}}-\frac{R r_{A} \cos \theta_{A}}{\sqrt{R^{2}+\left(z_{A}-z\right)^{2}}}
$$

In the denominator this will be further simplified to $u=\sqrt{R^{2}+\left(z_{A}-z\right)^{2}}$.

The pressure can now be approximated by:

$$
p(A, \omega)=j k \frac{\hat{p} R^{2}}{2 \pi} \int_{-\infty}^{\infty} \frac{e^{-j k\left(\sqrt{R^{2}+\left(z_{A}-z\right)^{2}}+\sqrt{R^{2}+z^{2}}\right)}}{\sqrt{R^{2}+\left(z_{A}-z\right)^{2}}\left(R^{2}+z^{2}\right)} \int_{0}^{2 \pi} e^{j k\left(\frac{R r_{A} \cos \theta_{A}}{\sqrt{R^{2}+\left(z_{A}-z\right)^{2}}}\right)} d \theta d z
$$

or, by using the Bessel function $J_{0}(z)=\int_{0}^{2 \pi} e^{-i z \cos \theta} d \theta$ :

$$
p(A, \omega)=j k \frac{\hat{p} R^{2}}{2 \pi} \int_{-\infty}^{\infty} \frac{e^{-j k\left(\sqrt{R^{2}+\left(z_{A}-z\right)^{2}}+\sqrt{R^{2}+z^{2}}\right)}}{\sqrt{R^{2}+\left(z_{A}-z\right)^{2}}\left(R^{2}+z^{2}\right)} J_{0}\left(\frac{k R r_{A}}{\sqrt{R^{2}+\left(z_{A}-z\right)^{2}}}\right) d z
$$

The approximate solution for this integral is:

$$
p(A, \omega)=\hat{p}(1+i) R\left(\frac{\pi k}{2\left(R^{2}+z_{A}^{2} / 4\right)^{3 / 2}}\right)^{1 / 2} J_{o}\left(\frac{k R r_{A}}{\sqrt{R^{2}+z_{A}^{2} / 4}}\right) e^{-2 j k \sqrt{R^{2}+z_{A}^{2} / 4}}
$$


The amplitude of the pressure in the receiver point $A$ will be:

$$
|p(A, \omega)|=\hat{p} R\left(\frac{\pi k}{\left(R^{2}+z_{A}{ }^{2} / 4\right)^{3 / 2}}\right)^{1 / 2} J_{o}\left(\frac{k R r_{A}}{\sqrt{R^{2}+z_{A}{ }^{2} / 4}}\right)
$$

\subsubsection{Pressure in the origin based on the approximation}

\section{Source in the origin}

With the source and the receiver positioned in the origin $(0,0,0)$ of a full cylinder $\left(r_{A}=0, z_{A}=0\right.$ and $\left.\theta_{m}=2 \pi\right)$, formula (8.19) will result in (8.9):

$$
|p(O, \omega)|=\hat{p} R\left(\frac{\pi k}{\left(R^{2}\right)^{3 / 2}}\right)^{1 / 2}=\hat{p} \sqrt{\frac{\pi k}{R}}
$$

\section{Source position outside the origin}

For a source position at some (small) distance $-r_{A}$ from the centre axis, the pressure in the point-symmetrical focusing point at $r_{A}$ from the centre axis can be derived. When using the first to terms of the Taylor series, the result will be identical to (8.6), meaning that moving the source away from the centre axis will not influence the pressure in the focussing point.

Derivation:

Much like the distance $u$ in (8.14), the distance $s$ can be written as a function of $r_{A}$. For $z_{A}=0$ :

$$
s^{2}=R^{2}+r_{A}^{2}+2 R r_{A} \cos \theta_{A}+z^{2}
$$

For small $r_{A}$ this can be approximated by:

$$
s^{2}=R^{2}+z^{2}+2 R r_{A} \cos \theta_{A}
$$

The first two terms of the Taylor series will be used for the approximation of $s$ in the phase:

$$
s=\sqrt{R^{2}+z^{2}}+\frac{R r_{A} \cos \theta_{A}}{\sqrt{R^{2}+z^{2}}}
$$

In the denominator this will be further simplified to $s=\sqrt{R^{2}+z^{2}}$.

Using (8.22) and (8.15) (for $z_{A}=0$ ), the pressure can now be approximated by:

$$
p(A, \omega)=j k \frac{\hat{p} R^{2}}{2 \pi} \int_{-\infty}^{\infty} \frac{e^{-j k 2 \sqrt{R^{2}+z^{2}}}}{\left(R^{2}+z^{2}\right)^{3 / 2}} \int_{0}^{2 \pi} d \theta d z
$$

where the positive and negative terms of (8.15) and (8.22) disappear and the result is identical to the formulation with the source and the receiver in the focal

As shown for the reflection from the sphere in par. 7.4.2, this result is only a firstorder approximation. The spherical aberration can be estimated by taking also into account the third term of the Taylor series. 


\section{Derivation:}

Including the third term in (8.15) and (8.22):

$$
\begin{aligned}
& u=\sqrt{R^{2}+z^{2}}-\frac{R r_{A} \cos \theta_{A}}{\sqrt{R^{2}+z^{2}}}-\frac{\left(R r_{A} \cos \theta_{A}\right)^{2}}{2\left(R^{2}+z^{2}\right)^{3 / 2}} \\
& s=\sqrt{R^{2}+z^{2}}+\frac{R r_{A} \cos \theta_{A}}{\sqrt{R^{2}+z^{2}}}-\frac{\left(R r_{A} \cos \theta_{A}\right)^{2}}{2\left(R^{2}+z^{2}\right)^{3 / 2}}
\end{aligned}
$$

This will result in:

$$
\begin{aligned}
& p(A, \omega)=j k \frac{\hat{p} R^{2}}{2 \pi} \int_{-\infty}^{\infty} \frac{e^{-j k 2 \sqrt{R^{2}+z^{2}}}}{\left(R^{2}+z^{2}\right)^{3 / 2}} \int_{0}^{2 \pi} e^{j k \frac{R^{2} r_{A}{ }^{2}}{\left(R^{2}+z^{2}\right)^{3 / 2} \cos ^{2} \theta_{A}} d \theta d z} \\
& \text { Using } \frac{1}{2 \pi} \int_{0}^{2 \pi} e^{j z \cos ^{2} \varphi} d \varphi=J_{o}\left(\frac{z}{2}\right): \\
& p(A, \omega)=j k \hat{p} R^{2} \int_{-\infty}^{\infty} \frac{e^{-j k 2 \sqrt{R^{2}+z^{2}}}}{\left(R^{2}+z^{2}\right)^{3 / 2}} J_{o}\left(\frac{k R^{2} r_{A}{ }^{2}}{2\left(R^{2}+z^{2}\right)^{3 / 2}}\right) d z
\end{aligned}
$$

Taking into account the third term of the Taylor series will result in:

$$
p(A, \omega)=j k \hat{p} R^{2} \int_{-\infty}^{\infty} \frac{e^{-j k 2 \sqrt{R^{2}+z^{2}}}}{\left(R^{2}+z^{2}\right)^{3 / 2}} J_{o}\left(\frac{k R^{2} r_{A}{ }^{2}}{2\left(R^{2}+z^{2}\right)^{3 / 2}}\right) d z
$$

This integral is solved numerically. Figure 8.4 shows the reduction of the pressure $f\left(r_{A}\right)=p\left(r_{A}, 0,0\right) / p(0,0,0)$ as a function of the relative distance $r_{A} / \sqrt{\lambda R}$.

For $r_{A}<0,75 \sqrt{\lambda R}$ this function can be estimated by:

$$
f\left(r_{A}\right) \approx \cos \left(2,2 \frac{r_{A}^{2}}{\lambda R}\right)
$$

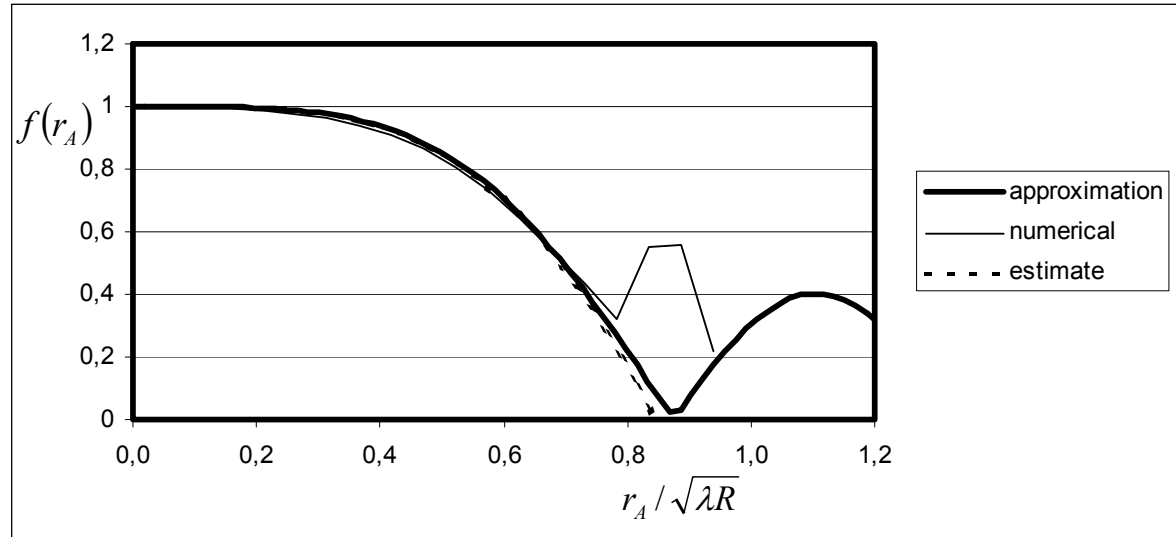

Figure 8.4. The reduction factor $f\left(r_{A}\right)$ of the pressure in the focusing point depending on the relative distance $r_{A} / \sqrt{\lambda R}$ from the centre axis of a cylinder. Numerical calculation using (6.18), approximate solution using (8.27) and estimate using (8.28a). 
Note: From numerical experiments it was found that for $x<0,75 \sqrt{\lambda R}$ and $R \cos \left(1-\theta_{m}\right)>\lambda$ a fair approximation of the reduction factor can be found for opening angles smaller than $\theta_{m}=\pi / 2$ :

$$
f\left(r_{A}\right) \approx \cos \left(1,4 \theta_{m} \frac{r_{A}^{2}}{\lambda R}\right)
$$

The results show that indeed the third term of the Taylor series explains the reduction of the pressure for sources moving away from the centre. For practical purposes the estimates (8.28a) and (8.28b) will be sufficiently accurate.

\subsubsection{Pressure at the axis of the cylinder}

The pressure along the axis of the circular cylinder will be considered here for the situation with the source in the centre of the cylinder.

Along the axis: $r_{A}=0$. Formula (8.19) will become:

$$
\begin{aligned}
|p(A, \omega)| & =\hat{p} \sqrt{\frac{R^{3}}{\left(R^{2}+z_{A}^{2} / 4\right)^{3 / 2}}} \cdot \sqrt{\frac{\pi k}{R}} \\
& =\hat{p} \sqrt{\frac{\pi k}{R}}\left(1+\left(\frac{z_{A}}{2 R}\right)^{2}\right)^{-3 / 4}
\end{aligned}
$$

The function $f\left(z_{A}\right)=\left(1+\left(\frac{z_{A}}{2 R}\right)^{2}\right)^{-3 / 4}$ is a slowly decreasing function for an increasing distance of the receiver $A$ on the $z$-axis from the source, see figure 8.5 .

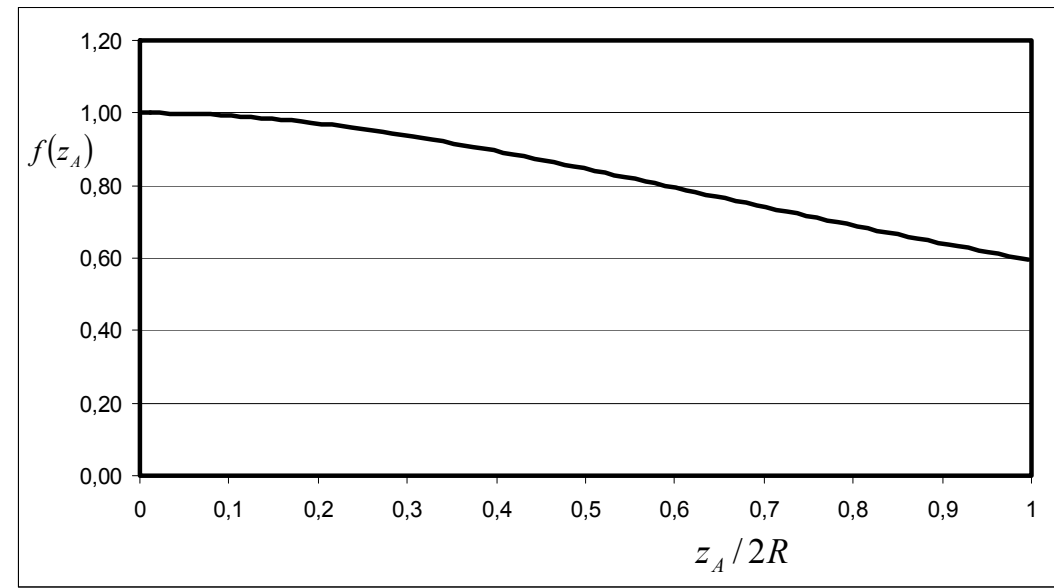

Figure 8.5. The reduction factor $f\left(z_{A}\right)$ of the pressure in the focusing point depending on the relative distance $z_{A} / 2 R$ along the $z$-axis $\left(r_{A}=0\right)$ of a cylinder according to (8.29). Numerical result using (8.2) is identical. 


\subsubsection{Pressure in the radial plane of the cylinder}

The pressure in the radial plane through the origin of the circular cylinder will be considered here for the situation with the source in the centre of the cylinder For that case: $z_{A}=0$. Formula (8.19) will become:

$$
|p(A, \omega)|=-\hat{p} \sqrt{\frac{\pi k}{R}} \cdot J_{o}\left(k r_{A}\right)
$$

A calculation example is shown in figure 8.6. The result of $(8.30)$ is very close to the numerical solution. The geometrical sound field is approximately the average of the oscillating sound field, except when it is close to the focal line $\left(r_{A}=0\right)$ where the geometrical solution is not defined.

For small $r_{A}$ the main lobe of (8.30) can be approximated by:

$$
|p(A, \omega)|=-\hat{p} \sqrt{\frac{\pi k}{R}} \cdot \cos \left(\frac{k r_{A}}{\sqrt{2}}\right)
$$

The width of this main lobe, defined by the $-3 \mathrm{~dB}$ points will be: $-\frac{\pi}{4}<\frac{k r_{A}}{\sqrt{2}}<\frac{\pi}{4}$

This corresponds to: $-0,18 \lambda<r_{A}<0,18 \lambda$

Thus for a full cylinder, the width of the lobe will be approx. $0,35 \lambda$

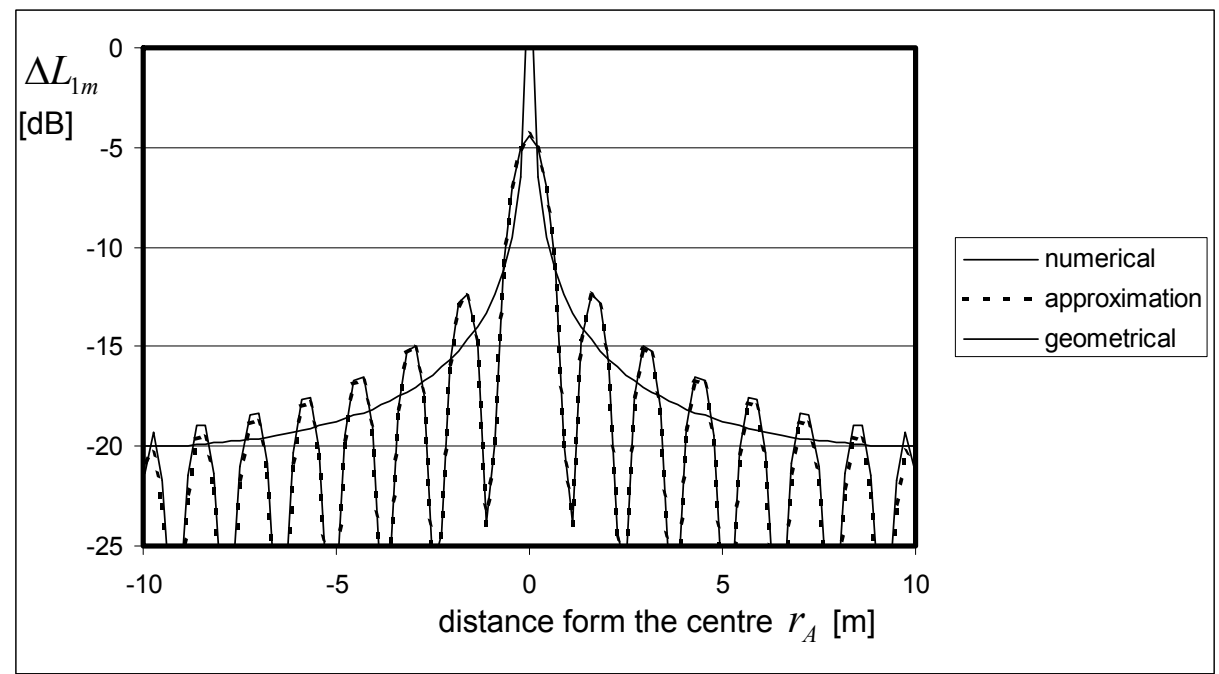

Figure 8.6. Calculation example of the oscillating sound field in a cylinder $z_{s}=z_{A}=0$. Shown is the SPL with the numerical solution using (8.2), the approximate solution using (8.30) and the geometrical sound field using (5.20), all relative to the SPL at $1 \mathrm{~m} . R=10 \mathrm{~m}$, $f=125 \mathrm{~Hz}$

Beyond the point where interference starts to occur, the sound field may be approximated by the geometrical sound field. To approximate the distance from the intersection point of the geometrical field to the focusing point, the first zero of (8.31) can be used:

$$
r_{A}=0,35 \lambda
$$




\section{$\underline{\text { 8.4 Diffuse reflections in a cylinder }}$}

In the previous sections a full reflection is assumed against a hard surface.

As with the sphere, in the case of perfect diffuse reflections, a random phase relation at each reception point can be assumed. Therefore energy will be added instead of pressure.

The intensity in a point at angle $\varphi$ and distance $u$ from surface element $d S$ can be calculated from:

$$
I=\frac{I_{i} d S \cos \alpha \cos \varphi}{\pi s^{2} u^{2}}
$$

Where $I_{i}=$ the intensity of the incident wave:

$$
I_{i}=\frac{\hat{p}^{2}}{2 \rho c \cdot s^{2}}
$$

The total pressure results from integration over $d S$ :

$$
p_{r m s}^{2}=\int_{s} \frac{\hat{p}^{2}}{2 \pi} \frac{\cos \alpha \cos \varphi}{s^{2} u^{2}} d S
$$

If we consider the pressure in the origin $\left(s=u=\sqrt{R^{2}+z^{2}}, \cos \alpha=\cos \varphi=\frac{R}{u}\right)$ this reduces to:

$$
p_{r m s}^{2}=\frac{\hat{p}^{2}}{2 \pi} R^{3} \int_{0}^{2 \pi} \int_{-\infty}^{\infty} \frac{1}{u^{6}} d z d \theta=\hat{p}^{2} R^{3} \int_{-\infty}^{\infty} \frac{1}{\left(R^{2}+z^{2}\right)^{3}} d z
$$

It can be shown that the solution of this integral is:

$$
p_{r m s}^{2}=\frac{3}{8} \pi \frac{\hat{p}^{2}}{R^{2}}
$$

This is considerably lower than the specular reflection in the focusing point (8.9).

Formula (8.36) is the solution for the full cylinder. For the half cylinder it will be:

$$
p_{r m s}^{2}=\frac{3}{16} \pi \frac{\hat{p}^{2}}{R^{2}}
$$

This is a factor $\frac{3}{16} \pi$ (approximately $2 \mathrm{~dB}$ ) lower than the diffuse sound pressure in the hemisphere (7.94).

As with the spherical reflector, the problem of the focusing due to reflections from a cylinder could theoretically be solved by generating diffuse reflections. The practical possibilities will be discussed in chapter 13.1. 


\section{Chapter 9}

\section{The sound field in an ellipsoid}

The remarkable property of the ellipsoid is that it has 2 focal points. A monopole sound source in one focal point will cause focusing in the other focal point. In practical cases of room acoustics, this shape poses the highest risk of adverse focusing effects.

However, the mathematics of ellipsoidal shapes is rather complex. A closed form solution or approximation of the sound field is not available at this moment.

Nevertheless, for completeness, the ellipsoidal shape will be discussed here. Some numerical examples will be given as well as a numerical approximation of the pressure in the focal point.

\section{$\underline{9.1 \text { Geometry of the ellipsoid }}$}

A numerical solution using (6.19) requires the calculation of $\cos \alpha, \cos \varphi$ (with $\alpha, \varphi$ being the angles of the incident and reflected sounds respectively) and for that, the normal to the surface must be calculated.

An ellipsoid can be described by:

$$
\frac{x^{2}}{a^{2}}+\frac{y^{2}}{b^{2}}+\frac{z^{2}}{c^{2}}=1
$$

The normal to the surface at position $(x, y, z)$ will be:

$$
\bar{n}=\left(\frac{x}{a^{2}}, \frac{y}{b^{2}}, \frac{z}{c^{2}}\right)
$$

A special case is: $a=b=c$. In that case the ellipsoid is a sphere.

Here a prolate ellipsoid will be assumed: $a=b$ and $c>a$.

The distance from either of the two focal points to the centre of the ellipsoid is:

$$
z=\sqrt{c^{2}-a^{2}}
$$

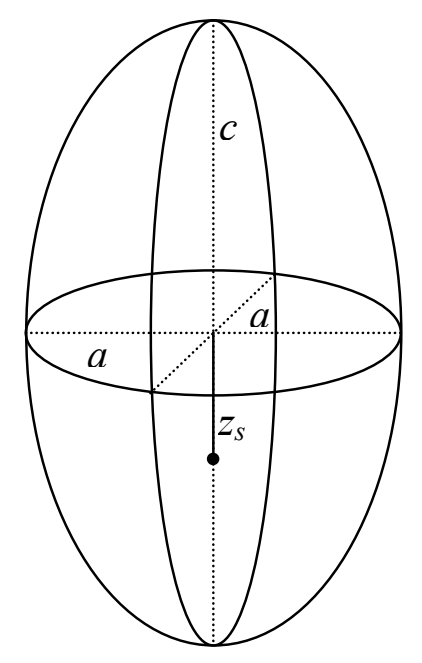

Figure 9.1. Prolate ellipsoid with axis $a$ and $c(c>a)$. 


\subsection{Pressure in the focal point of an ellipsoid}

When the source is in one focal point and the receiver is in the other, $\cos \alpha$ and $\cos \varphi$ in (6.19) will be smaller than 1 (as is the case for the sphere).

A closed form solution for the pressure in the focal point is not available at this moment.

Numerical experiments have been performed with prolate ellipsoids with a $c / a$ ratio ranging from 1 to 2 (in 0.05 steps). The calculation is performed for frequencies 250 $\mathrm{Hz}, 500 \mathrm{~Hz}, 1 \mathrm{kHz}$ and $2 \mathrm{kHz}$ and for $a=b=2 \mathrm{~m}$. The source is positioned in one of the focal points. From (6.19), with the use of (9.2), the pressure in the other focal point is calculated and compared to the pressure in the focal point of a sphere $(c / a=1)$.

The decrease of pressure as a function of increasing $c / a$ ratio is shown in figure 9.2. From regression it is found that the decrease of pressure can be approximated by a power function:

$$
|p| \approx 2 \hat{p} k\left(\frac{a}{c}\right)^{1,4}
$$

The coefficient of determination $R^{2}$ of this approximation is 0,998 . The deviation is within $3 \%$ for $1<c / a<2$.

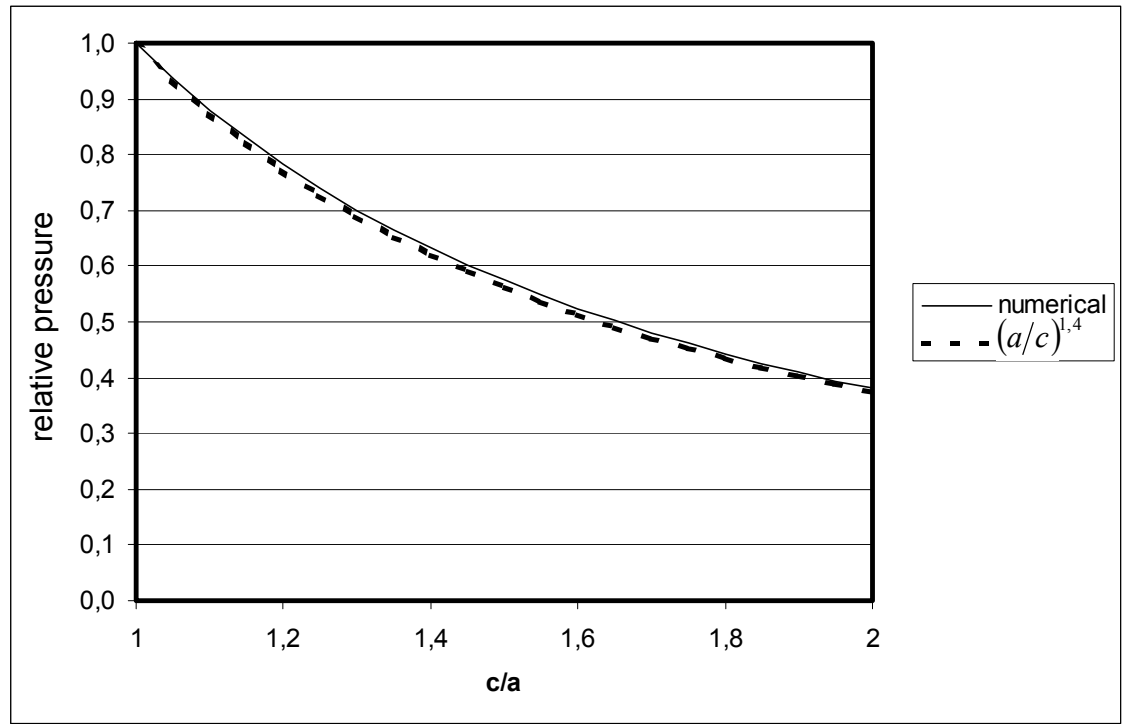

Figure 9.2. Decrease of the pressure in the focal point depending on the ratio c/a of a prolate ellipsoid, relative to the pressure in a sphere with c/a=1. Numeric calculation (with (6.19), using (9.1) and (9.2)) and approximation (9.4) 


\subsection{Example for the sound pressure field in an ellipsoid.}

A calculation example of the pressure field in an ellipsoid is shown in figure 9.3.
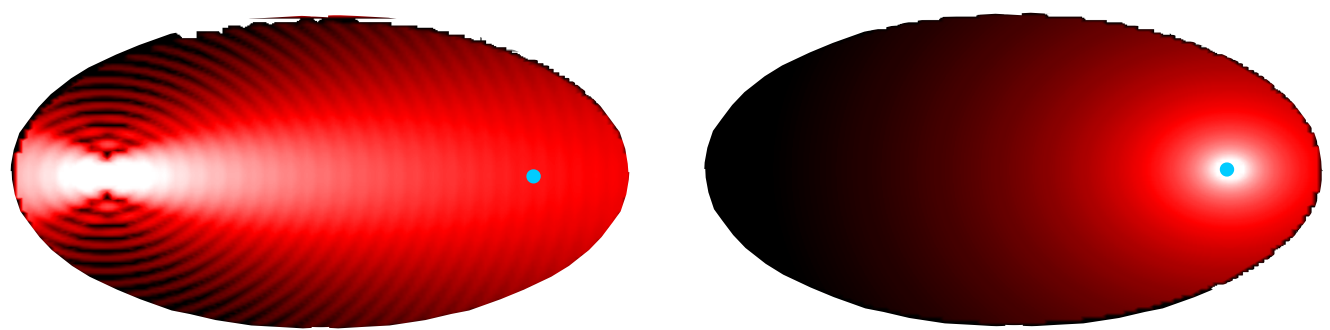

Figure 9.3. Calculation example of the sound pressure in an ellipsoid $(a=2,07 \mathrm{~m}, c=2,93 \mathrm{~m})$ with source $(\hat{p}=1 \mathrm{~N} / \mathrm{m})$ in the right focal point (Blue dot). Right: direct sound. Left: reflected sound. Frequency: $1000 \mathrm{~Hz}$. Color range: white $\geq+20 \mathrm{~dB}$, black $\leq-10 \mathrm{~dB}$.

It is interesting that the reflected sound pressure is relatively high along the long axis of the ellipsoid. Figure 9.4 shows the results when only half of the ellipsoid exists. Especially when the source is outside the ellipsoid, there seems to be relatively high pressure in a narrow range along the axis.
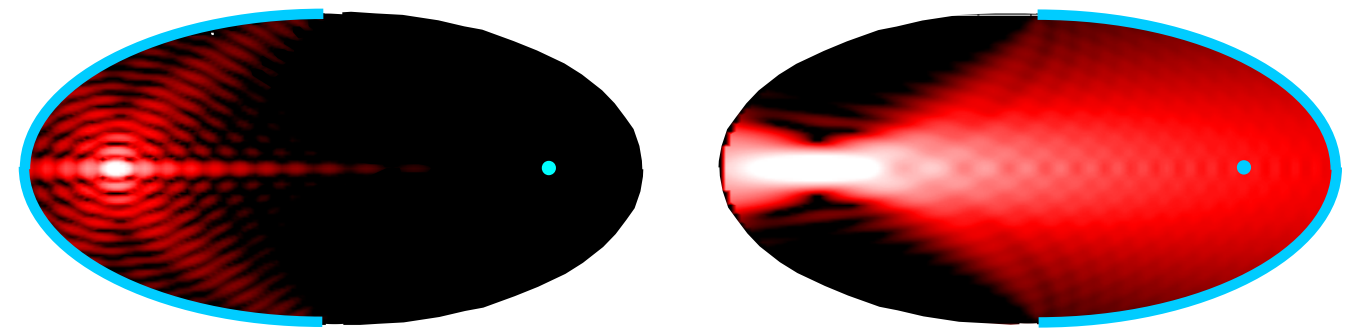

Figure 9.4. Calculation example of the reflected sound pressure in a half ellipsoid $(a=2,07 m$, $c=2,93 \mathrm{~m})$ with source $(\hat{p}=1 \mathrm{~N} / \mathrm{m})$ in the right focal point (Blue dot). Right: half ellipsoid on the right side (blue line); left: half ellipsoid on the left side (blue line). Frequency: $1000 \mathrm{~Hz}$. Color range: white $\geq+20 \mathrm{~dB}$, black $\leq-10 \mathrm{~dB}$. 


\section{Chapter 10}

\section{The sound field from a double-curved rectangular surface}

In sections 7,8 , and 9 the sound field is described for spherical, cylindrical and ellipsoidal shapes. In this section, an approximation will be given of the reflection from a double-curved surface of a rectangular (projected) shape.

\subsection{Integral formulation for the double-curved surface}

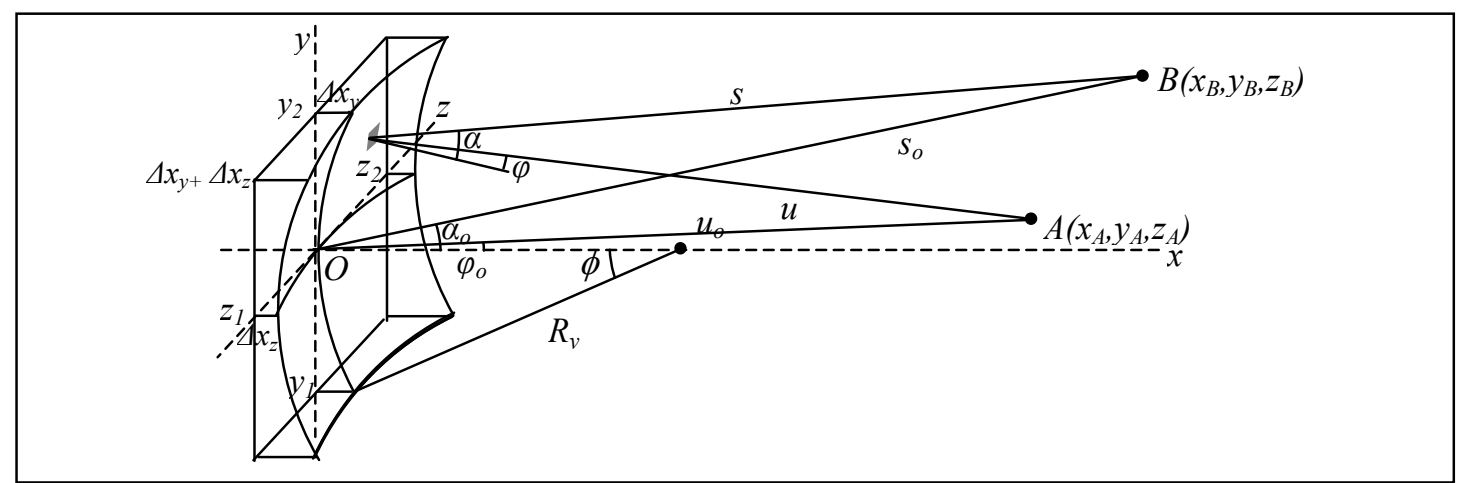

Figure 10.1. Coordinates and symbols used for the calculation of the reflected sound field from a rectangular, double-curved surface.

Figure 10.1 shows the model with a rectangular, curved segment, where the curvature can be different in $y$ - and $z$-direction. The origin is in the middle of the surface.

For a sound source in $B$ the general formulation of the sound pressure in $A$, using $d S=d y d z$ and integrating over the dimensions of the rectangle $y_{1}-y_{2}$ and $z_{1}-z_{2}$ will be, from (6.18):

$$
p(A, \omega)=\frac{\hat{p}}{4 \pi} \int_{z=z_{1}}^{z_{2}} \int_{y=y_{1}}^{y_{2}}\left(\frac{1+j k s}{s} \cos \alpha+\frac{1+j k u}{u} \cos \varphi\right) \frac{e^{-j k(s+u)}}{s u} d y d z
$$

In this formulation a small error is made in the surface: the surface $d S=d y d z$ is in fact the projected surface on the $x=0$ plane. That means that (10.1) is only valid for small opening angles $\phi$ in the $y$-and $z$-direction.

\subsection{Pressure in the centre of the double-curved surface}

As a first check, the pressure in the centre of the double-curved surface will be derived. In the special case the source and receiver are in the centre of curvature, both for the $y$ - and $z$-direction (this means that the double-curved surface is a sphere segment), $s=u=R$ and $\cos \alpha=\cos \varphi=1$, so (10.1) will reduce to:

$$
p(A, \omega)=\frac{\hat{p}}{2 \pi} \int_{z=z_{1}}^{z_{2}} \int_{y=y_{1}}^{y_{2}} \frac{1+j k R}{R^{3}} e^{-j k 2 R} d y d z
$$

In the far field $(k R>>1)$ :

$$
p(A, \omega)=\frac{j k \hat{p}}{2 \pi R^{2}}\left(y_{2}-y_{1}\right)\left(z_{2}-z_{1}\right) e^{-j k 2 R}=\frac{j k \hat{p} S}{2 \pi R^{2}} e^{-j k 2 R}
$$

where $S$ is the total projected area of the double-curved surface. This formulation corresponds with (7.3), when accounting for the appropriate reflecting surface. 
The next step will be to make further approximations to be able to solve (10.1) for other positions of source and receiver and different curvatures in the $y$ - and $z$ directions.

\subsection{Approximate solution for the double-curved surface}

Due to the small size and curvature of the element (compared to the distance of source and receiver) some of the distances to $d S$ and the angles may be approximated by the distance and angle to the centre of the sphere segment.

With this assumption the double integral (10.1) can be reduced into two single integrals.

The following approximations for the amplitude part of the integral can be used:

$$
\begin{aligned}
& u \approx u_{0}, \cos \varphi \approx \cos \varphi_{0} \\
& s \approx s_{0}, \cos \alpha \approx \cos \alpha_{0}
\end{aligned}
$$

this will result in:

$$
p(A, \omega)=\frac{\hat{p}}{4 \pi \cdot u_{0} s_{0}}\left(\frac{1+j k s_{0}}{s_{0}} \cos \alpha_{0}+\frac{1+j k u_{0}}{u_{0}} \cos \varphi_{0}\right) \iint e^{-j k(u+s)} d y d z
$$

The phase function is much more sensitive. Therefore, more exact expressions for $u$ and $s$ as function of $y$ and $z$ are needed. With the curvature in the $x$-direction $u$ can be approximated:

$$
\begin{aligned}
u^{2} & =\left(x_{A}-\left(\frac{y^{2}}{2 R_{y}}+\frac{z^{2}}{2 R_{z}}\right)\right)^{2}+\left(y_{A}-y\right)^{2}+\left(z_{A}-z\right)^{2} \\
& =x_{A}^{2}+y_{A}^{2}+z_{A}^{2}-2 x_{A}\left(\frac{y^{2}}{2 R_{y}}+\frac{z^{2}}{2 R_{z}}\right)-2 y_{A} y-2 z_{A} z+\left(\frac{y^{2}}{2 R_{y}}+\frac{z^{2}}{2 R_{z}}\right)^{2}+y^{2}+z^{2}
\end{aligned}
$$

The curvature in the $y$ and $z$ dimension can be separately entered by $R_{y}$ and $R_{z}$.

Assuming that $y$ and $z$ are small compared to $u_{0}$, the last three terms can be neglected. Furthermore: $u_{0}^{2}=x_{A}^{2}+y_{A}^{2}+z_{A}^{2}$

Using the first two terms of the series, $u$ and $s$ can be approximated by:

$$
\begin{aligned}
& u \approx u_{0}-\frac{x_{A}}{u_{0}}\left(\frac{y^{2}}{2 R_{y}}+\frac{z^{2}}{2 R_{z}}\right)-\frac{y_{A}}{u_{0}} y-\frac{z_{A}}{u_{0}} z \\
& s \approx s_{0}-\frac{x_{B}}{s_{0}}\left(\frac{y^{2}}{2 R_{y}}+\frac{z^{2}}{2 R_{z}}\right)-\frac{y_{B}}{s_{0}} y-\frac{z_{B}}{s_{0}} z
\end{aligned}
$$

The phase function will be:

$$
-j k(u+s)=-j k\left(u_{0}+s_{0}\right)+j C_{1} y^{2}+j C_{2} y+j C_{3} z^{2}+j C_{4} z
$$

with: $C_{1}=k\left(\frac{x_{A}}{2 u_{0} R_{y}}+\frac{x_{B}}{2 s_{0} R_{y}}\right)$,

$$
\begin{array}{ll}
C_{1}=k\left(\frac{x_{A}}{2 u_{0} R_{y}}+\frac{x_{B}}{2 s_{0} R_{y}}\right), & C_{2}=k\left(\frac{y_{A}}{u_{0}}+\frac{y_{B}}{s_{0}}\right) \\
C_{3}=k\left(\frac{x_{A}}{2 u_{0} R_{z}}+\frac{x_{B}}{2 s_{0} R_{z}}\right), & C_{4}=k\left(\frac{z_{A}}{u_{0}}+\frac{z_{B}}{s_{0}}\right)
\end{array}
$$

In this formula $C_{1}$ and $C_{3}$ are related to the curvature of the surface while $C_{2}$ and $C_{4}$ are related to the deviation of source and receiver from the specular direction: 


$$
\begin{aligned}
& C_{1}=\frac{k}{2 R_{y}}\left(\cos u_{o}+\cos s_{o}\right), \\
& C_{2}=k\left(\sin u_{0, z=0}+\sin s_{0, z=0}\right),
\end{aligned}
$$

with the sinus taken from the projection on the $z=0$ surface

$$
\begin{aligned}
& C_{3}=\frac{k}{2 R_{z}}\left(\cos u_{o}+\cos s_{o}\right), \\
& C_{4}=k\left(\sin u_{0, y=0}+\sin s_{0, y=0}\right),
\end{aligned}
$$

with the sinus taken from the projection on the $y=0$ surface

The double integral in (10.1) and (10.4) can now be replaced by two single integrals.

$$
p(A, \omega)=\frac{\hat{p} \cdot e^{-j k\left(u_{0}+s_{0}\right)}}{4 \pi \cdot u_{0} s_{0}}\left(\frac{1+j k s_{0}}{s_{0}} \cos \alpha_{0}+\frac{1+j k u_{0}}{u_{0}} \cos \varphi_{0}\right) \int e^{j\left(C_{1} y^{2}+C_{2} y\right)} d y \int e^{j\left(C_{3} z^{2}+C_{4} z\right)} d z
$$

with $C_{1}, C_{2}, C_{3}$ and $C_{4}$ as indicated for (10.8), see text block.

The integrals are almost identical for the $y$ and $z$ direction.

The single integral integrating over $d y$ (from $y_{1}$ to $y_{2}$ ) can be solved:

$$
\begin{aligned}
\int_{y_{1}}^{y_{2}} e^{j\left(C_{1} y^{2}+C_{2} y\right)} d y= & \sqrt{\frac{\pi}{8 C_{1}}(1+j) \cdot e^{-j \frac{C_{2}{ }^{2} C_{1}}{1}} \cdot} \\
& {\left[\operatorname{erf}\left(\frac{(1-j)}{\sqrt{8 C_{1}}}\left(2 C_{1} y_{2}+C_{2}\right)\right)-\operatorname{erf}\left(\frac{(1-j)}{\sqrt{8 C_{1}}}\left(2 C_{1} y_{1}+C_{2}\right)\right)\right] }
\end{aligned}
$$

For this integral a closed form solution exists [Abramowitz70] (in general form, so the $a, b$, $x$ and $z$ used here are not related to the problem above):

$$
\int e^{j\left(a x^{2}+b x\right)} d x=\sqrt{\frac{j \pi}{4 a}} \cdot e^{-j \frac{b^{2}}{4 a}} \cdot \operatorname{erf}\left(\sqrt{-j a} x-\frac{j b}{2 \sqrt{-j a}}\right)=\sqrt{\frac{\pi}{8 a}}(1+j) \cdot e^{-j \frac{b^{2}}{4 a}} \cdot \operatorname{erf}\left((1-j)\left(\sqrt{\frac{a}{2}} x+\frac{b}{\sqrt{8 a}}\right)\right)
$$

with the error function: $\operatorname{erf}(z)=\frac{2}{\sqrt{\pi}} \int_{0}^{z} e^{-t^{2}} d t$

where $z$ can be a complex number.

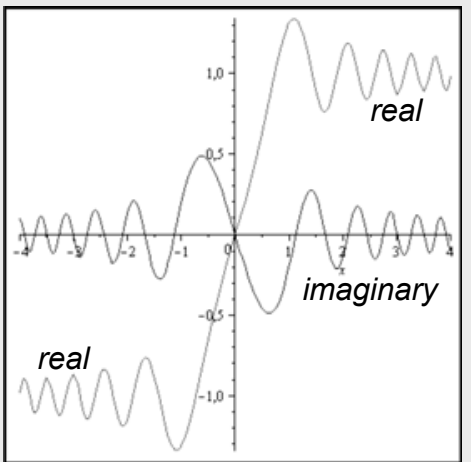

A graphical representation of the error function with $z=(1+j) x$ is presented in figure 10.2.

The error function can be approximated by two expansion series, for small and large $x$.

This closed form solution can be used for solving (10.10)

Figure 10.2. Real and imaginary parts of the function $z=(1+j) x$ with real $x$

An example of this error-difference function is shown in figure 10.3. 
This error-difference function has a maximum for $C_{2}=0$ (for $\frac{y_{A}}{u_{0}}=-\frac{y_{B}}{s_{0}}$ ) in the case that $y_{1}=-y_{2}$. This reflects the specular reflection direction.

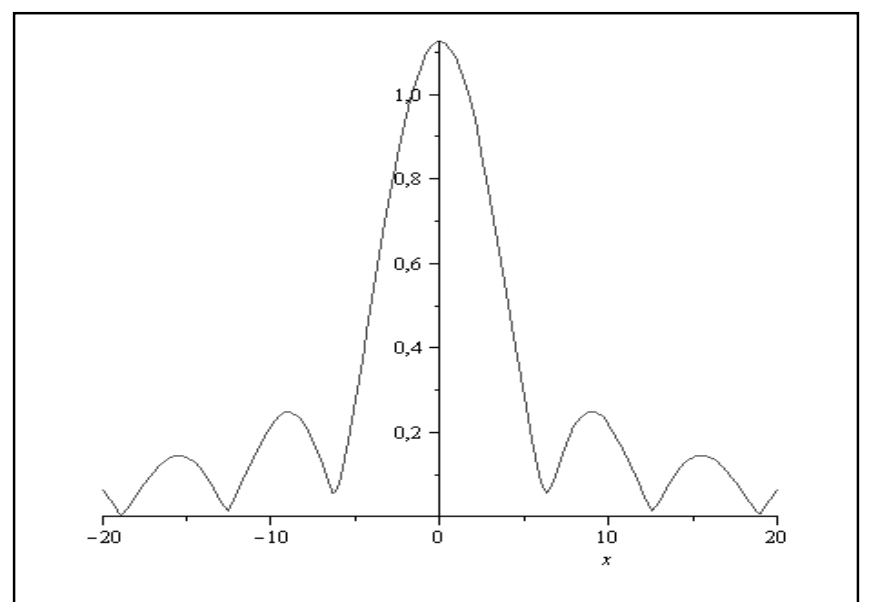

Figure 10.3. Example of the difference between two (shifted) error functions (amplitude as a function of $x)$ : $\operatorname{erf}\left(\frac{1+j}{\sqrt{8}}(1-x)\right)-\operatorname{erf}\left(\frac{1+j}{\sqrt{8}}(-1-x)\right)$

Since the solution for $\mathrm{z}$ will be similar (integrating from $z_{1}$ to $z_{2}$, where $z_{1}=-z_{2}$ ) to the solution for $y$, the integral solution of the pressure in $A$ will be:

$$
\begin{aligned}
& p(A, \omega)=\frac{\hat{p} \cdot e^{-j k\left(u_{0}+s_{0}\right)}}{4 \pi \cdot u_{0} s_{0}}\left(\frac{1+j k s_{0}}{s_{0}} \cos \alpha_{0}+\frac{1+j k u_{0}}{u_{0}} \cos \varphi_{0}\right) . \\
& \sqrt{\frac{\pi}{8 C_{1}}}(1+j) \cdot e^{-j \frac{C_{2}^{2}}{4 C_{1}}} \cdot\left[\operatorname{erf}\left(\frac{(1+j)}{\sqrt{8 C_{1}}}\left(2 C_{1} y_{2}-C_{2}\right)\right)-\operatorname{erf}\left(\frac{(1+j)}{\sqrt{8 C_{1}}}\left(2 C_{1} y_{1}-C_{2}\right)\right)\right] \\
& \cdot \sqrt{\frac{\pi}{8 C_{1}}}(1+j) \cdot e^{-j \frac{C_{4}^{2}}{4 C_{3}}} \cdot\left[\operatorname{erf}\left(\frac{(1+j)}{\sqrt{8 C_{3}}}\left(2 C_{3} z_{2}-C_{4}\right)\right)-\operatorname{erf}\left(\frac{(1+j)}{\sqrt{8 C_{3}}}\left(2 C_{3} z_{1}-C_{4}\right)\right)\right]
\end{aligned}
$$

If the surface is symmetrical around the origin: $y_{1}=-y_{2}$ and $z_{1}=-z_{2}$ :

$$
\begin{aligned}
& p(A, \omega)=\frac{\hat{p} \cdot e^{-j k\left(u_{0}+s_{0}\right)}}{4 \pi \cdot u_{0} s_{0}}\left(\frac{1+j k s_{0}}{s_{0}} \cos \alpha_{0}+\frac{1+j k u_{0}}{u_{0}} \cos \varphi_{0}\right) \cdot \\
& \sqrt{\frac{\pi}{8 C_{1}}}(1+j) \cdot e^{-j \frac{C_{2}^{2}}{4 C_{1}}} \cdot\left[\operatorname{erf}\left(\frac{(1+j)}{\sqrt{8 C_{1}}}\left(2 C_{1} y_{2}-C_{2}\right)\right)+\operatorname{erf}\left(\frac{(1+j)}{\sqrt{8 C_{1}}}\left(2 C_{1} y_{2}+C_{2}\right)\right)\right] \\
& \cdot \sqrt{\frac{\pi}{8 C_{1}}}(1+j) \cdot e^{-j \frac{C_{4}^{2}}{4 C_{3}}} \cdot\left[\operatorname{erf}\left(\frac{(1+j)}{\sqrt{8 C_{3}}}\left(2 C_{3} z_{2}-C_{4}\right)\right)+\operatorname{erf}\left(\frac{(1+j)}{\sqrt{8 C_{3}}}\left(2 C_{3} z_{2}+C_{4}\right)\right)\right]
\end{aligned}
$$

This formulation can be seen as an extension of the Fraunhofer formula for curved surfaces. A calculation example of (10.13) compared to the numerical solution of (6.19), is given in figure 10.4 . 

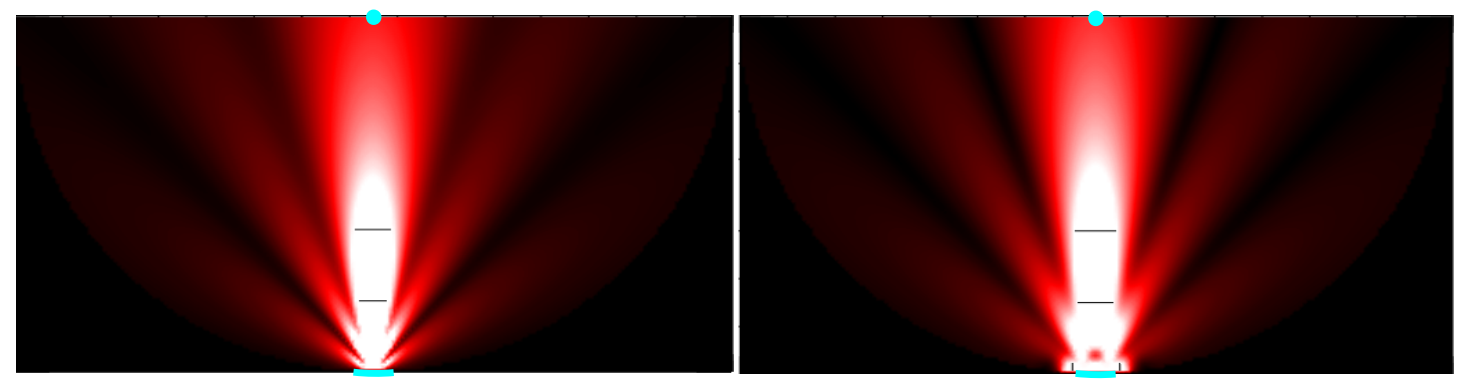

Figure 10.4. Reflected sound pressure by a rectangular curved surface of $1 \times 1 \mathrm{~m}$ (Blue line), radius $R=10 \mathrm{~m}$, source $(\hat{p}=1 \mathrm{~N} / \mathrm{m})$ in centre point (Blue dot), $k=9,2(500 \mathrm{~Hz})$; Area shown: 10 m x 20 m. Left: Approximation using (10.13), Right: Numerical result using (6.19). Color range: linear scale, white: $\geq 0,1 \mathrm{~Pa}$, black: $0 \mathrm{~Pa}$.

At a short distance, some deviations from the numerical solution can be seen. At sufficient distance from the reflecting surface, the pressure of the reflected sound field can be calculated with sufficient accuracy using (10.13).

Although the error function can numerically be calculated, a further simplification of (10.13) would be helpful. However, at this moment a further simplification is not available. 


\section{SECTION IV. VERIFICATION OF THE KIRCHHOFF INTEGRAL}

The theory on sound focusing presented in section III is based on the Kirchhoff Integral, as explained in chapter 6 . A verification of this theory is performed with experiments described in chapter 11.

\section{Chapter 11 \\ Experiments}

To verify the theoretical amplification in the focal point a small experimental setup was made. This setup is described in par.11.2. To obtain the field sound pressure relative to the direct pressure from the sound source, impulse response measurements were performed. The measurement method is described in par. 11.1.

\section{$\underline{11.1 \text { Measurement method }}$}

To obtain the pressure in the focal point (relative to the pressure at $1 \mathrm{~m}$ from the source) both the pressure due to the reflection and the direct sound have to be measured. As for the reflected sound, we are only considering the first reflection (see also chapter 6 ). Measuring both the direct sound and the first reflection within the same measurement is done by measuring the impulse response function and time separation by applying identical time windows over the direct signal and over the reflected signal as shown schematically in figure 11.1 .

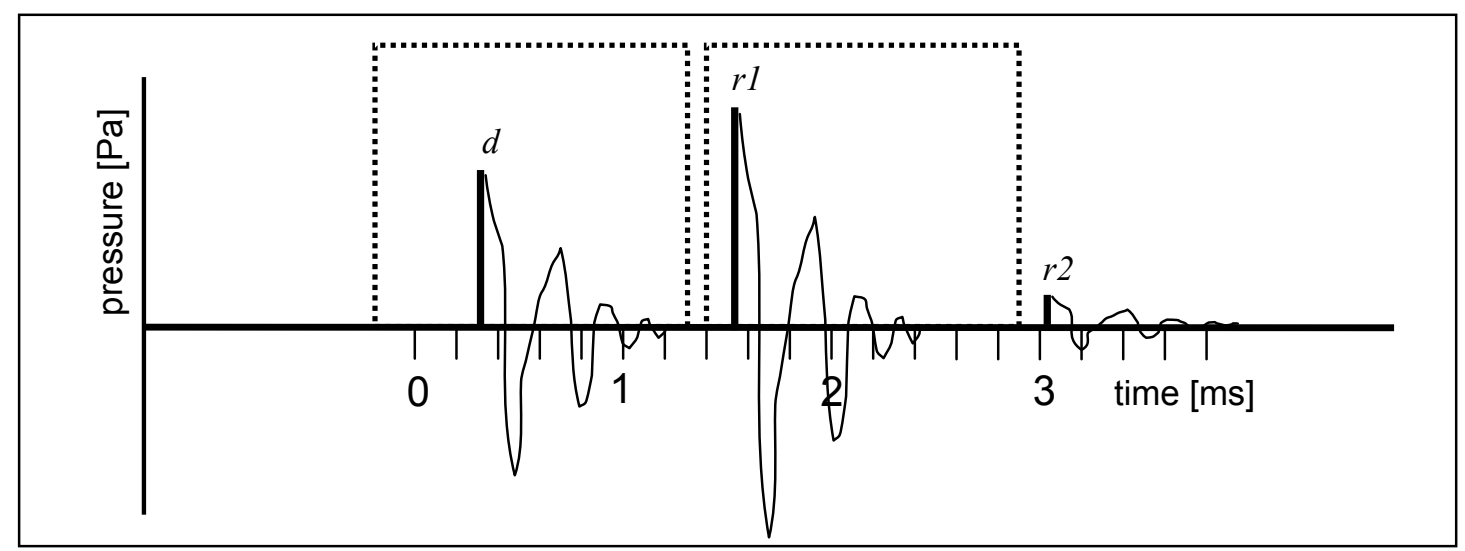

Figure 11.1. Schematic representation of the expected impulse response from the measurement setup. Time $t=0 \mathrm{~ms}$ corresponds to the moment the impulse leaves the tip of the source. $d=$ direct sound, $r 1=$ first reflection, $r 2=$ second reflection or internal reflection from the source. Dotted: time windows over direct and reflected sound.

\subsubsection{Sound source}

To be able to compare the results to numerical calculations the source has to be omni-directional. This is also relevant because the reference, the direct sound, is measured at one microphone position, so for a distinct direction. This makes the measurement sensitive to deviations from ideal omni-directionality. Ways to obtain some degree of omni-directional sources are:

- the use of spark sources; the disadvantage is the poor reproducibility and risk for non linear effects; 
- the use of multiple loudspeakers (dodecahedron) for different directions; the advantage is the possibility of using two channel methods (using the input to the loudspeaker and the microphone signal) to determine the impulse response from MLS signal or sweep, avoiding non-linearity; the disadvantage is the very limited and rather frequency-dependent omni-directionality;

- the use of a point source with very small dimension, by coupling a driver to a conical element narrowing the opening. The advantages are the possibility of using two channel methods and the omni-directionality up to high frequencies; the disadvantages are the low sound power (due to the small opening) and the internal reflection between opening and driver.

The last technique is used, amongst others, by the author for room acoustics scale model research and offers the best possibilities for performing the verification.

However, the internal reflection, which is sufficiently suppressed for normal use in room acoustics, had to be removed for this particular purpose, to obtain an exact measurement result. This is done by increasing the distance between the driver and the opening of the source, so that the internal reflection is delayed and arrives at the microphone after the first reflection from the sample. The internal reflection (from the tip back to the driver and then again back to the tip) will be outside the measurement window (see $r_{2}$ in figure 11.1).

The source used is a Vifa XT-200 driver that is connected to a $1 \mathrm{~m}$ long copper tube (internal diameter $=5 \mathrm{~mm}$ ) with a small cone (see also figure 11.7). The tip has been made conical. This diameter corresponds to $1 / 8$ wavelength at $8500 \mathrm{~Hz}$. Up to this frequency a rather good omni-directionality can be expected.

The frequency response of the source is given in figure 11.2. For frequencies up to $30 \mathrm{kHz}$, which covers the relevant frequency range, the source has sufficient sound power.

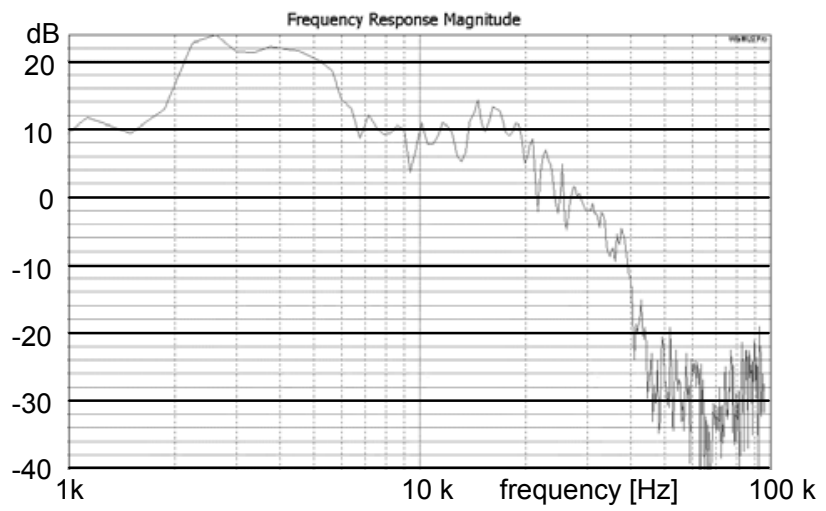

Figure 11.2. Frequency response of the sound source on axis direction.

The directional characteristics of this source are measured with a rotating table in an anechoic room. Figure 11.3 shows the measurement results in the radial and axial plane for the third-octave bands from $2 \mathrm{kHz}$ until $6.3 \mathrm{kHz}$. The maximum deviation is about $3 \mathrm{~dB}$. The dip at $180^{\circ}$ in the axial plane is caused by the shading of the driver.

\subsubsection{Microphone}

As for the sound source, the omni-directionality of the sensitivity of the microphone is also important, especially for the measurement of the direct signal.

The microphone used in this study was a free field microphone that was developed for Peutz for room acoustic scale model research. It has a $1 / 4$ " Sennheiser capsule and is shown in figure 11.4. The preamplifier is built into the wider base of the microphone.

The measured directional sensitivity of the microphone is shown in figure 11.5. The maximum deviation for the relevant opening angle is about $1 \mathrm{~dB}$.

The sensitivity is flat $\pm 1.5 \mathrm{~dB}$ until $25 \mathrm{kHz}$, then it falls off with $6 \mathrm{~dB} / o c t a v e$. 


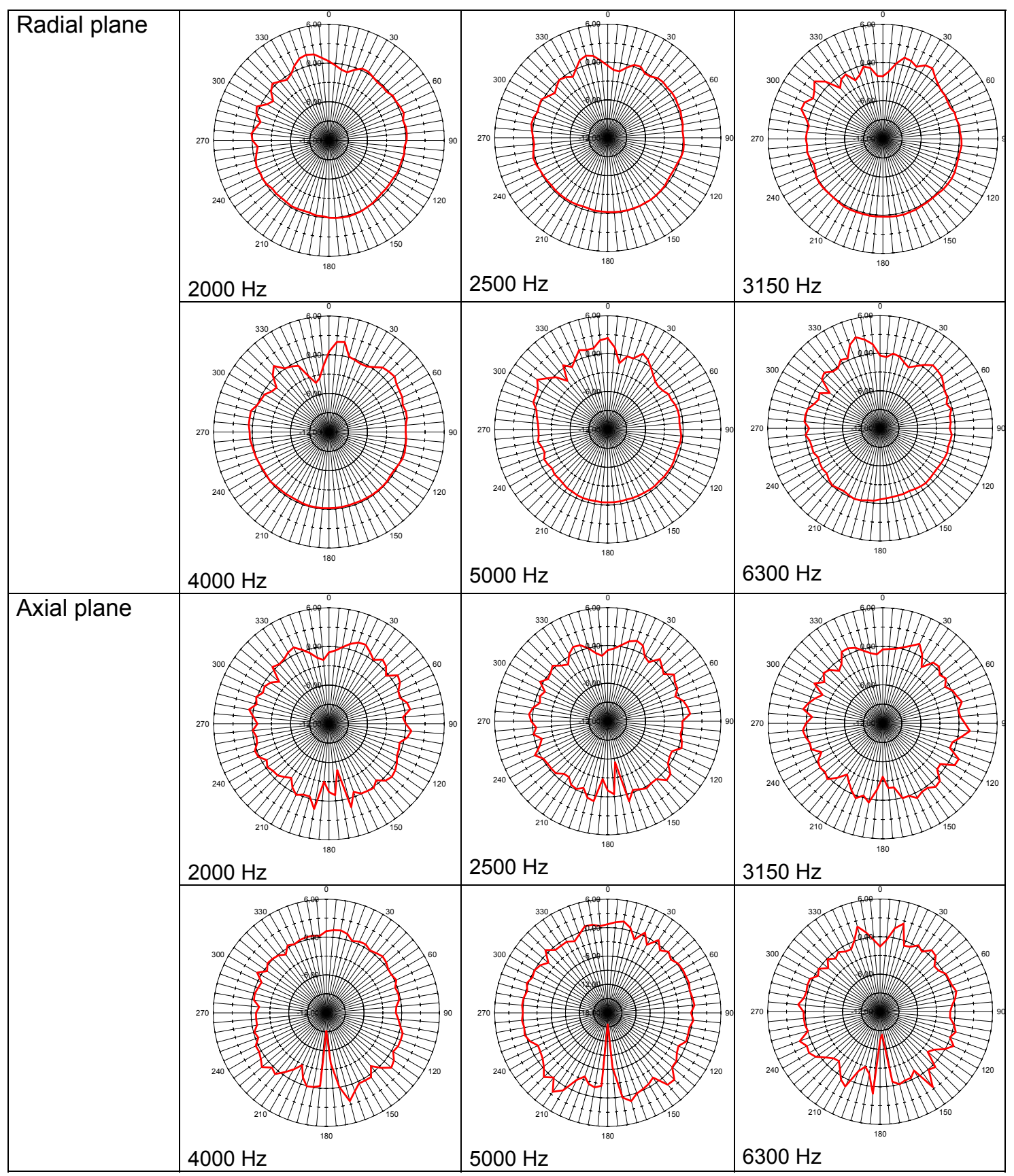

Figure 11.3. Polar plots of the sound source directionality in radial and axial plane. Measurement results as SPL in $d B$. Distance between centric solid lines is $6 \mathrm{~dB}$

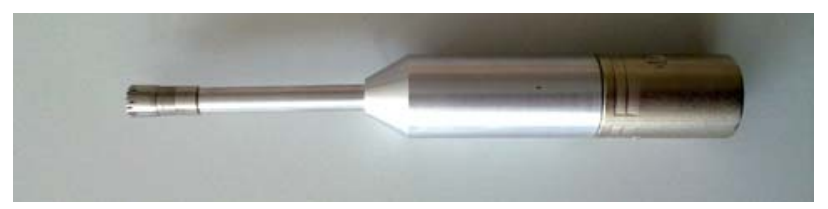

Figure 11.4. Picture of the room acoustics scale model microphone 


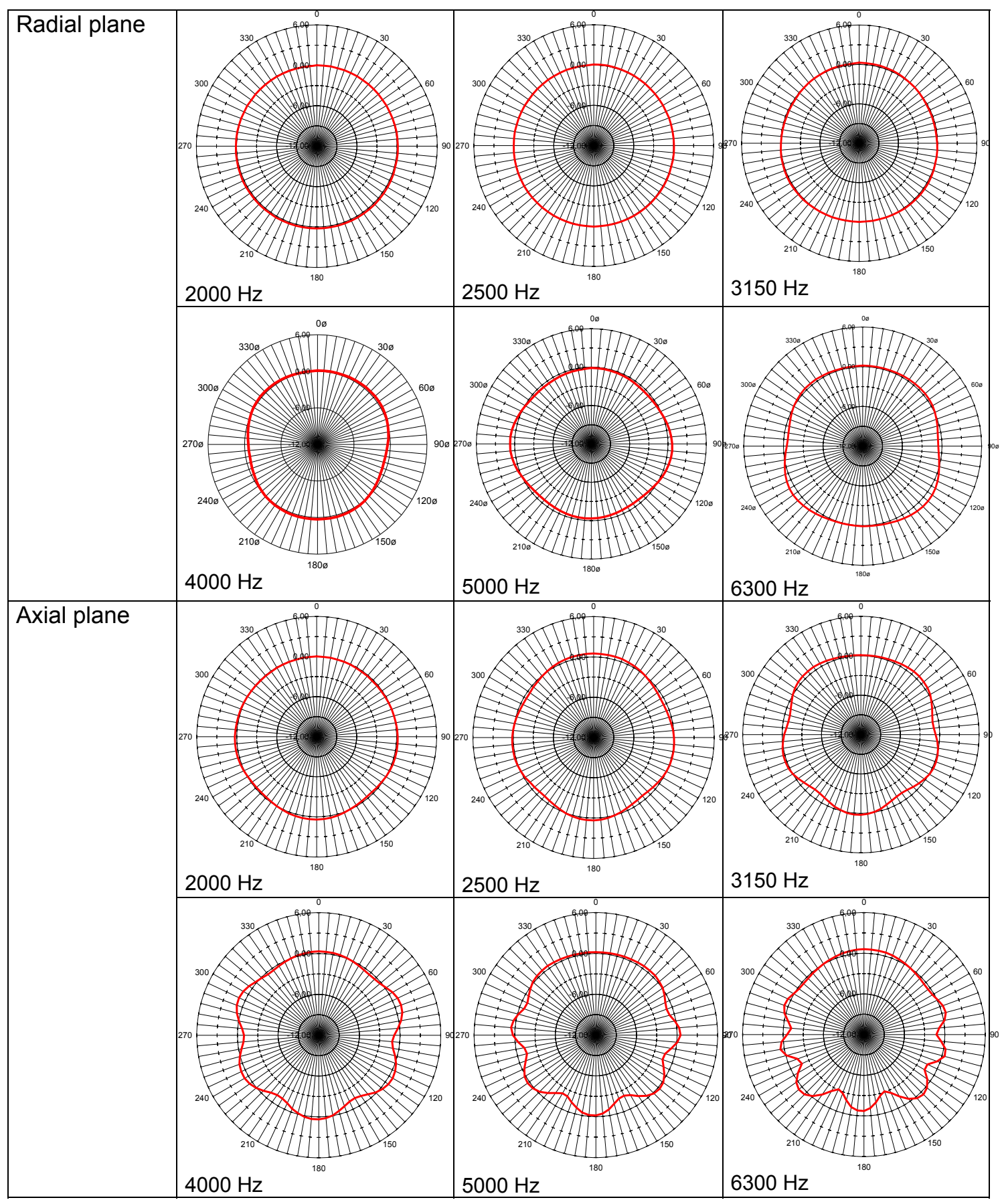

Figure 11.5. Polar plot of the microphone sensitivity in the radial and axial plane of the microphone. Measurement results as SPL in $\mathrm{dB}$. Distance between centric solid lines is $6 \mathrm{~dB}$

\subsubsection{Measuring system}

The periodic impulse response (PIR) is measured with a MLS (Maximum Length Sequence) signal and a WinMLS-2004 data-acquisition system. The sampling rate is $196 \mathrm{kHz}$. To reduce the influence of background noise, 16 measurements are averaged in the time domain. The time windows over direct sound and first reflection are rectangular windows with duration 1,5 ms. Multiple reflections (if there are any) are outside the measurement window. The signal within the time window is converted to frequency domain (FFT) and a third octave band synthesis is performed. Next, for 
each third octave band the difference between the direct and reflected signal is determined.

\section{$\underline{11.2 \text { Ellipsoidal reflector }}$}

The reflector used in the experimental setup consists of a half ellipsoid with the two focal points at a relatively small distance apart. By using an ellipsoidal shape the source and microphone can be at different positions and yet both can be in a focal point. This would not be the possible for a sphere. The use of a sphere would only be possible for positions of the source or microphone, or both, outside the centre. In that case, the focusing would be influenced by spherical aberration.

By using a small distance between the focal points the time delay between direct sound and reflected sound is maximized. Furthermore, the difference between SPL of the direct sound and SPL of the reflected sound will be influenced by the distance between the focal points. The SPL of the reflected sound is only moderately influenced by the distance between the focal points, see formula (9.4), while the direct sound is strongly influenced by this distance. By having focal points only a short distance apart, the levels of direct sound and reflected sound are in the same order of magnitude, which is important for measurement accuracy.

The model is especially CAD/CAM milled for this purpose, from a solid polyurethane block (Ebaboard PW 920), a material with a high density and excellent low surface porosity. The accuracy of the shape of the ellipsoid is approximately $0.01 \mathrm{~mm}$.

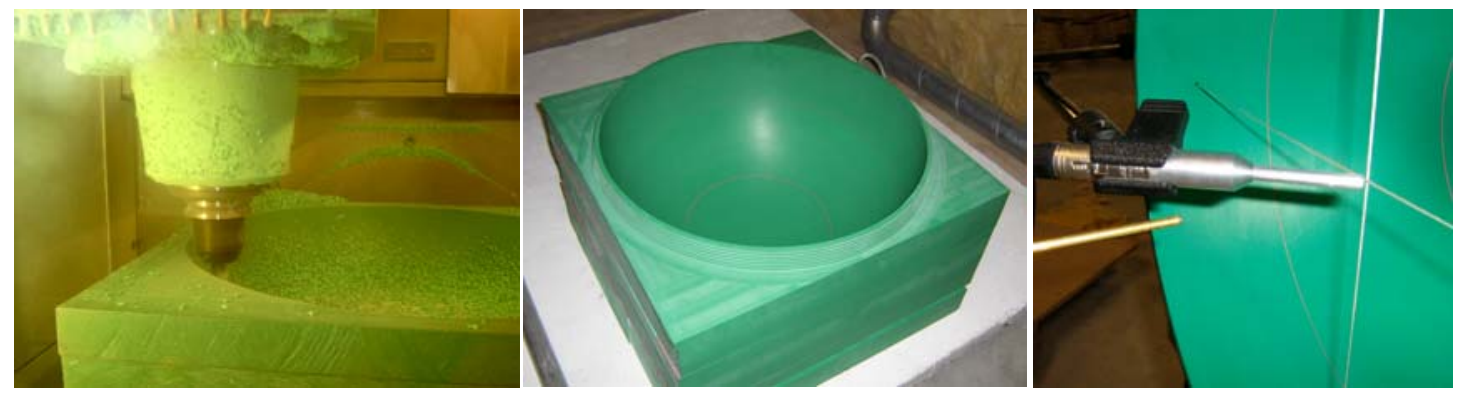

Figure 11.6. Polyurethane scale model. Left: production; middle: finished model; right: source and microphone positions

Figure 11.6 shows some pictures, giving an impression of the milling of the shape, the finished model and the measurement setup.

A section of the measurement setup is shown in figure 11.7.

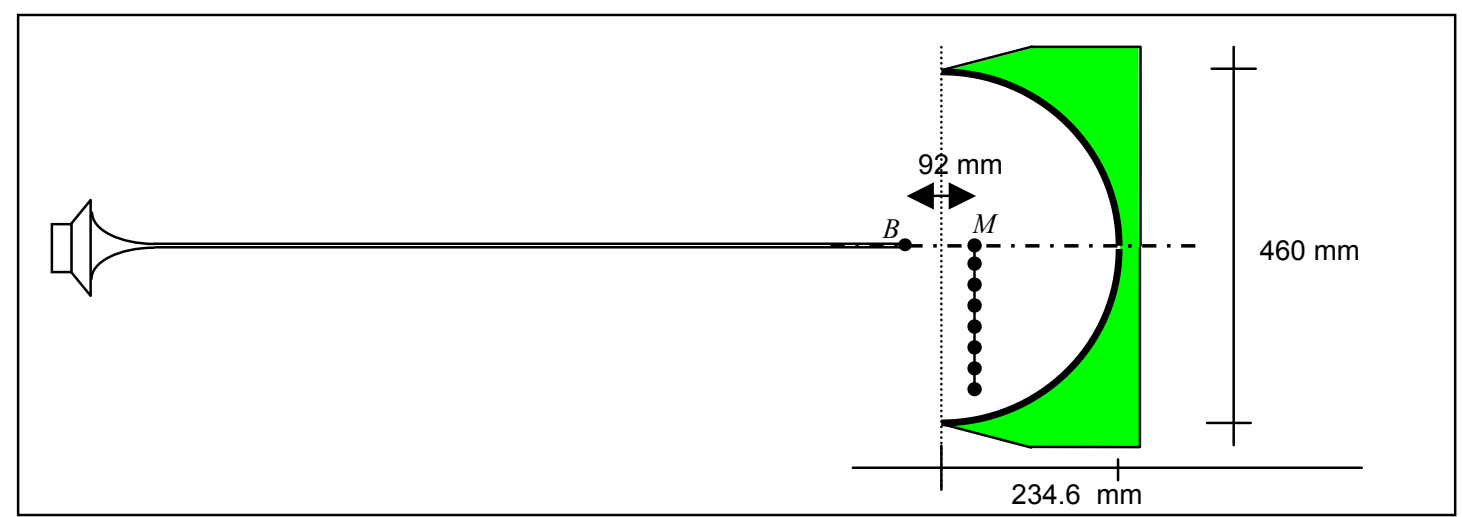

Figure 11.7. Section of the experimental setup (half-ellipsoid), $B$ is the tip of the source and $M$ is the microphone 


\subsection{Numerical approximation}

The sound pressure in the half ellipsoid, due to the source positioned in the left focal point as indicated in figure 11.7, is also calculated by numerical calculation of the Kirchhoff integral (6.19). The normal to the surface elements $d S$ is calculated using (9.2). The largest distance between the surface elements $d S$ is $1 / 8$ of the wavelength. The distance between the field points is also $1 / 8$ of the wavelength.

The numerical calculation with the Kirchhoff integral considers only the first reflection (see par.6.4). This corresponds to the applied measuring technique.

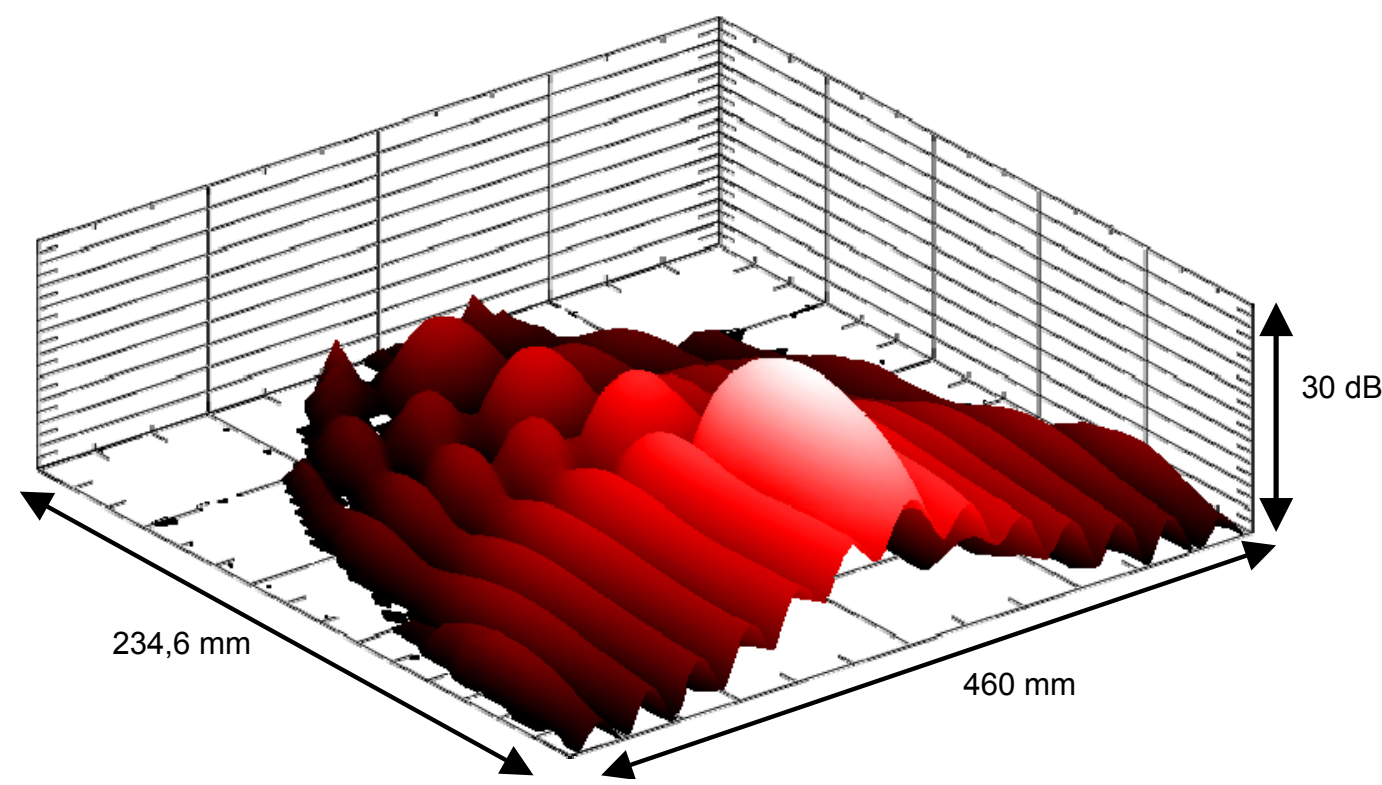

Figure 11.8. Reflected sound pressure field at $5 \mathrm{kHz}$ (pure tone) from a source at the position of figure 11.7. Calculated using (6.19). Calculated for the section of the half-ellipsoid indicated in figure 11.7

An impression of the calculated sound field in the axial plane (the area shown in figure 11.7, within the half ellipsoid) is shown for $5 \mathrm{kHz}$ (pure tone) in figure 11.8.

The calculated frequency dependent relative pressure in the focal point and the calculated relative pressure along a line through the focal point (see figure 11.7) are shown in figures 11.9 and 11.10 .

\section{$\underline{11.4 \text { Measurement results }}$}

The pressure is measured in the right focal point with the source in the left focal point (see figure 11.7). $\Delta L$, the increase of the reflected sound pressure level relative to the direct sound pressure level at the focal position, is shown in figure 11.9.

The measurement results corresponds to the calculation results within $1 \mathrm{~dB}$.

Figure 11.10 shows $\Delta L$, the pressure at the microphone positions along a line in the focal plane, as indicated in figure 11.7, relative to the direct sound pressure level at the focal position. Up until approximately $10 \mathrm{~cm}$ from the focal point, the measurement and calculation data correspond within approximately $1 \mathrm{~dB}$. For points further away the correspondence is a little less good. 


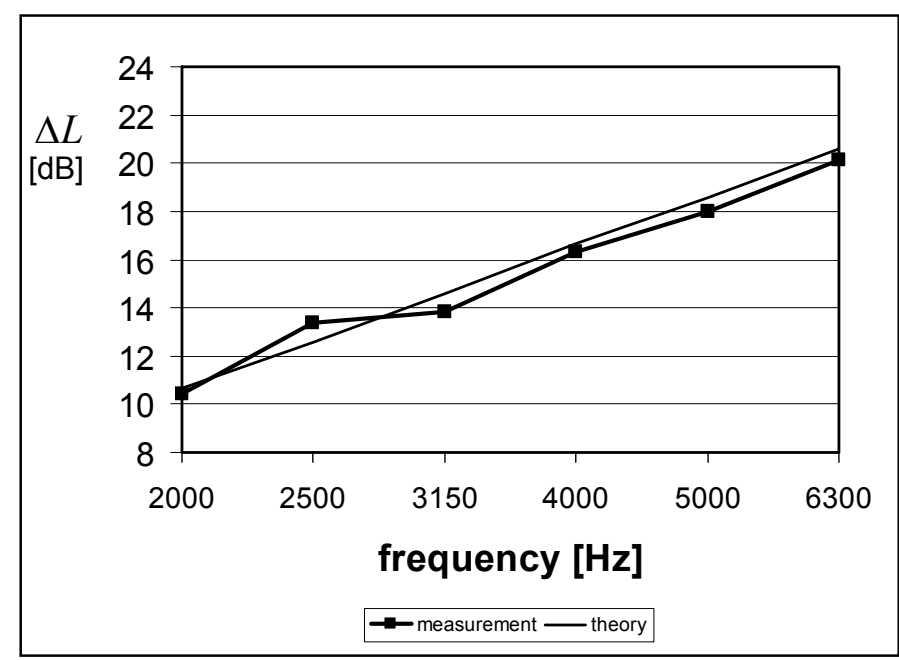

Figure 11.9. $\Delta L$, the SPL of the reflected sound in the focal point, relative to the direct sound, as a function of frequency. Continuous line: calculation result (6.19); line with markers: measurement results (both 1/3 oct values).

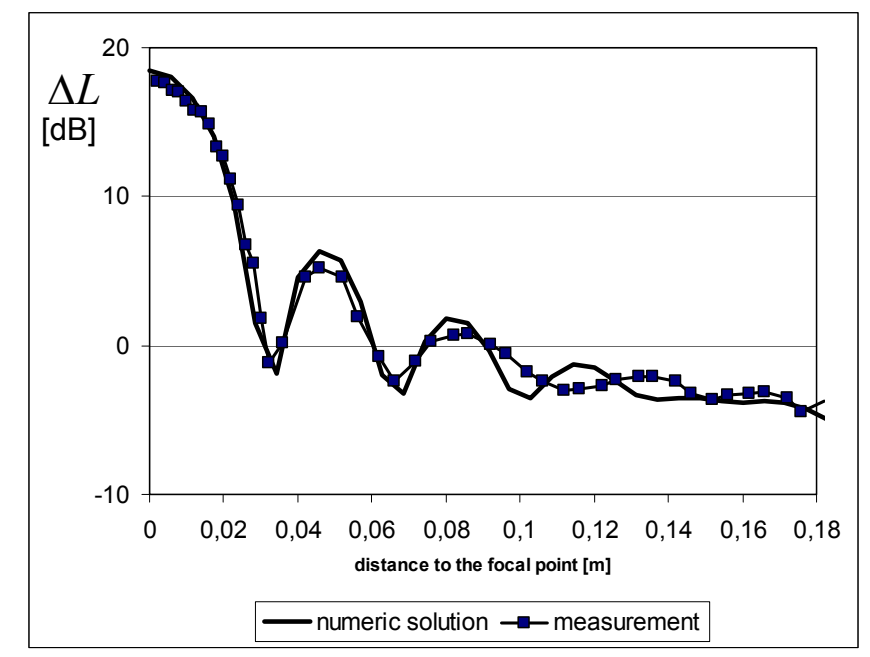

Figure 11.10. $\triangle L$, the SPL of the reflected sound along a line in the focal plane (see fig.11.7), relative to the direct sound in the focal point, at $5000 \mathrm{~Hz}$ (both $1 / 3$ oct). Continuous line: Numeric solution (6.19); line with markers: measurement result. 


\subsection{Measurement accuracy}

The method of relating the measured pressure of the reflected sound in the focal point to the measured pressure of the direct field eliminates systematic influences, such as microphone characteristics and non-flat frequency response of the source. The major factors contributing to the measurement accuracy are the deviations from the omni-directionality of the source and the microphone as presented in paragraph 11.1.

Assuming a maximum deviation of $3 \mathrm{~dB}$ for the source and $1 \mathrm{~dB}$ of the microphone the maximum total deviation of the measurement from the real value is $4 \mathrm{~dB}$. The correspondence between measurement and calculation result in figure 11.9 is well within that range. From performing ten measurements, the standard deviation of the SPL in the focal point (relative to the direct pressure) was determined. This standard deviation varied, for the third octave bands from $2 \mathrm{kHz}$ to $6,3 \mathrm{kHz}$, from 0,1 to $1,3 \mathrm{~dB}$.

The measurement accuracy will be reduced when part of the signal is outside the time window. At lager distances from the focal point (see figure 11.10) the measured average pressure level is correct but the interference patterns do not correspond. For positions outside the focal point the reflections will not arrive at the same moment, as in the focal point, but are distributed in time. Since the spread in time will be larger than the measuring window, some of the reflected energy will be outside of the measurement window, resulting in too low measured pressure.

\subsection{Conclusion}

It is concluded that, despite small differences, the measurement results show good agreement with the theory based on the Kirchhoff integral (6.19) for the calculation of the first reflection. 


\section{SECTION V \\ A PRACTICAL APPROACH ON ESTIMATION OF SOUND CONCENTRATION}

The theory of wave field calculation using the Kirchhoff Integral for the first reflection is applied to several shapes in section III. This rather theoretical work has practical implications that will be discussed in this section.

The first step will be the analysis of the design of the space and its reflecting surfaces. The most important formulas from section III are summarized in chapter 12, presenting a practical approach to the analysis of a room shape, e.g., in the design phase. The main goal here is to estimate the sound pressure level and to evaluate whether sound reducing measures are necessary.

Next step might be to reduce the sound focusing. Practical measures that can be used to reduce the focusing, and the efficiency of these possibilities, are discussed in chapter 13.

Chapter 14 presents a number of different cases of sound focusing. In a number of these cases the method presented in chapter 12 is applied.

When an analytical or numerical approach is hardly possible, e.g., because of the complex geometry, an alternative solution is to perform scale model research. In chapter 14 two cases of halls with severe sound focusing problems are described. In these cases the problems were investigated and solved with the use of scale model research.

\section{Chapter 12 \\ Engineering approach}

\section{$\underline{12.1 \text { Geometrical sound field vs. wave field approach }}$}

To make a prediction of the sound pressure level it must be determined whether the sound field can be considered a geometrical sound field following the rules of geometrical acoustics (chapter 5), or whether actual focusing is to be expected. In the latter case the wave field approximation formulas presented in section III have to be applied; in the first case the geometrical formulas will suffice.

\section{Position of the focusing point}

As a first step a geometrical investigation is needed regarding:

- $\quad$ the position of the focusing point or focusing line;

- $\quad$ the expected focusing positions, given the (known) source position(s).

To determine the position of the focusing point due to reflection from a spherical or circular cylindrical shape, a line is to be constructed between the source $B$ and the intersection of the curved surface with its (rotational) axis, as indicated in figure 12.1. The focusing point will be on the specular line (this is according to Snel's law: angle of reflection is equal to the angle of incidence).

A source position in the centre will have its focusing point at that same position.

For source positions close to the centre of a sphere or cylinder, the focusing position will be approximately point symmetrical to the centre. For source positions further away, the distance $w$ from the focusing point to the reflecting surface can be determined from the thin lens formula (5.6):

$$
\frac{1}{w}+\frac{1}{s}=\frac{2}{R \cos \theta}
$$


where $s: \quad$ distance from the source to the reflecting surface

$R: \quad$ radius of the sphere or cylinder

$\theta$ : $\quad$ angle of incidence

$w$ : distance from the focusing point to the reflecting surface

A source at an infinite distance from the reflector (incident plane wave) will have its focal point halfway between the centre and the surface $(w=R / 2)$.

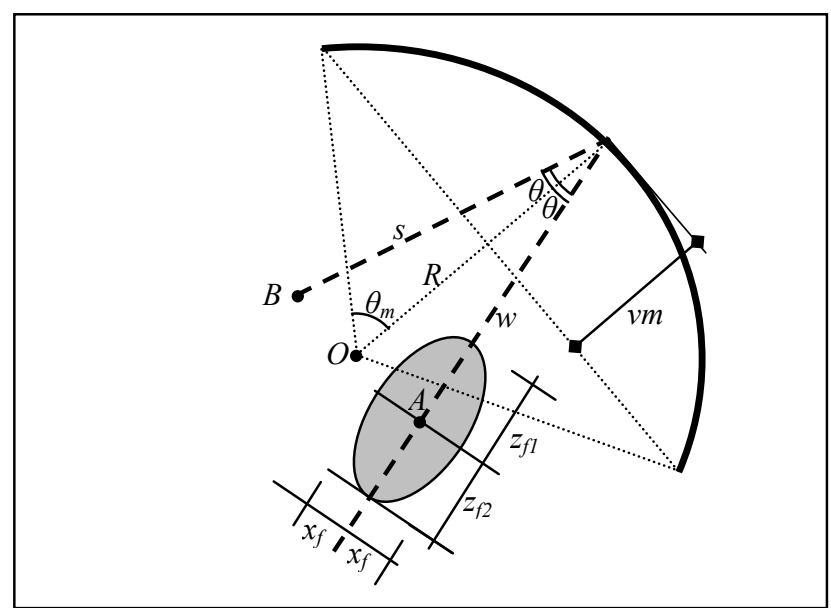

Figure 12.1. Indication of the focusing area (in grey) around the focusing point $A$, with dimensions $z_{f 1,2}$ and $x_{f}$.

For elliptical or ellipsoid shapes the positions of the focal points can be determined from (9.3):

$$
z=\sqrt{c^{2}-a^{2}}
$$

where: $z: \quad$ distance from the centre at the long axis

$a, c:$ the dimensions of the ellipsoid along the axes $(c>a)$, see figure 9.1

With the source in one focal point the focusing will occur in the other focal point.

If the focusing points will be sufficiently outside the audience areas (e.g., significantly under the floor level or above the audience level), a geometrical approach would be appropriate and sufficient.

An example of a situation where the geometrical approach is sufficient is given in chapter 14.4 (the dome reflection in the Royal Albert Hall).

\section{Size of the focusing area}

However, the question is: What is "sufficiently" outside the focusing positions? The focusing does not only occur in the focusing point, but in an area around that point, which will be defined as the focusing area where reflections from a significant number of wavelets are in phase and no interference is observed. This is the grey area in figure 12.1 .

The area outside this (grey) focusing area will be called the geometrical field. In this area the calculated monochromatical pressure shows a frequency-dependent interference pattern.

The transition point of the focusing area to the geometrical field can be defined as the location of the first interference dip in the pressure. This can be obtained from the approximations of the sound field covered in chapter 7 and 8 .

For a sphere or sphere segment, the distances $z_{f}$ from the focusing point to the intersection points of the wave approximation with the geometrical field along the specular line, can be derived from (7.53), see also (12.15): 


$$
z_{f 1,2}=\frac{R}{\frac{ \pm \lambda}{R\left(1-\cos \theta_{m}\right)}-\frac{2 z_{B}-R}{s}}-w
$$

where $\lambda \quad$ wavelength [m]

$z_{B} \quad$ the projection of the source position on the axis of the spherical segment

The two solutions $z_{f 1}$ and $z_{f 2}$ "in front" and "behind" the focusing point are different. In the radial direction, away from the reflection axis (see figure 12.1), the starting point of the geometrical field due to a sphere segment can be obtained from (7.67):

$$
x_{f}=\frac{\lambda w}{2 R \sin \theta_{m}}
$$

For example, for a hemisphere with a source close to the centre $(w=R)$, this results in

$$
x_{f}=0,5 \lambda
$$

This distance in a full cylinder is, from (8.32):

$$
x_{f}=0,35 \lambda
$$

It is noted that for sphere or cylinder segments a smaller opening angle $\theta_{m}$ leads to a larger focusing area. Apart from the opening angle, the size of the focusing area is primarily wavelength/frequency-related. The amplification at lower frequencies may be less than the amplification at higher frequencies, the focusing area is considerably larger at low frequencies (see also par. 12.3.2).

Two calculation examples of the determination of the focusing area are given in par.12.3.4.

With the method described above it is possible to determine the dimensions (width and length) of the focusing area, to estimate if the audience area is within this focusing area, and thus if a wave field approach is necessary or a geometrical approach of the sound field is sufficient.

Besides the method described above, there are two indicative alternative methods that can be considered to evaluate the necessity of a wave field approach.

The first alternative method of estimating if focusing might occur is to perform calculations with geometrical computer models. The example in par.14.1.4 shows that visualizing rays may enhance understanding of the situation. If the rays converge in a single point, a wave field approach is clearly necessary. The example in par. 14.6, however, shows that the calculation result in terms of calculated sound pressure level may be insufficient even to localize the focusing.

Contrary to the common method of modeling that uses surfaces that are as large as possible, to visualize sound concentration the segments have to be as small as possible.

A second alternative method of estimating if focusing occurs is to calculate the amplification in the audience area by the geometrical approach, as explained in the next paragraph. If the pressure at any position is more than e.g., $6 \mathrm{~dB}$ under the maximum pressure in the focusing point with the wave field approach (see par. 12.3.1), the geometrical approach is sufficient. A high pressure obtained from the geometrical approach indicates that a wave field approach is necessary. 


\subsection{Geometrical approach}

The pressure $\Delta L$ at a receiver position, relative to the direct sound at receiver position, can be calculated from the pressure $\Delta L_{r}$ due to the reflection from a plane surface at the same distance as the curved surface, adding the amplification caused by the curved surface $\Delta L_{c}(5.15)$ :

$$
\Delta L=\Delta L_{r}+\Delta L_{c}
$$

The sound pressure relative to the direct sound $\Delta L_{r}$ at the receiver position due to the reflection from a fully reflecting flat surface can be determined from (3.6):

$$
\Delta L_{r}=20 \lg \left(\frac{r_{d}}{s+u}\right)
$$

where $r_{d}$ is the distance between source and receiver, $s$ is the distance from the source to the reflecting surface and $u$ is the distance from the receiver position to the reflecting surface.

The geometrical amplification of the reflection due to the curvature of the reflecting surface $\Delta L_{c}$ can be determined from (5.16):

$$
\Delta L_{c}=-10 \lg \left(\left|\frac{1}{\left(\frac{1}{u}+\frac{1}{s}\right)} \frac{2}{R_{x} \cos \theta_{x}}-1\right|\right)-10 \lg \left(\left|\frac{1}{\left(\frac{1}{u}+\frac{1}{s}\right)} \frac{2}{R_{y} \cos \theta_{y}}-1\right|\right)
$$

In this formulation there is a separation made for the curvature in the $x$ - and $y$ direction. For spherical surfaces the radii are equal. For cylindrical surfaces only one term will be used $\left(R_{y} \rightarrow \infty\right)$. For ellipsoid surfaces different radii may be used (see the example of the Royal Albert Hall in par.14.4). In the case of elliptical shapes, the appropriate radius can be determined with (5.22), (see also the example in par.14.1.5).

By adding the $\Delta L_{c}$ from (5.16) and $\Delta L_{r}$ from (3.6), the SPL of the reflection from the curved surface relative to the direct sound $\Delta L$ is obtained.

Both $\Delta L$ and $\Delta L_{c}$ may be used to evaluate the echo, (see chapter 12.4).

A high $\Delta L_{c}$ (e.g., higher than the maximum pressure in the focusing point according to par. 12.3 minus $6 \mathrm{~dB}$ ) indicates that the receiver position is within the focusing area (for $u \rightarrow w: \Delta L_{c} \rightarrow \infty$ ) and a wave field approach has to be applied. 


\subsection{Wave field approach}

In chapter 7 a wave field approach is presented for calculating the sound field from a spherical reflector. It is found that, apart from the interference pattern, at a sufficient distance from the focusing point the sound field can be described by geometrical methods. For room acoustical purposes, the wave theoretical approach is only necessary for determining the pressure in the focusing point and focusing area.

In this paragraph, the most relevant results of the pressure at the focus, in the focusing plane and along the specular line of reflection are summarised and illustrated. This enables an estimate of the sound pressure level in practical situations.

\subsubsection{Pressure at the focusing point}

The situation will be considered of a hard spherical or circular cylindrical reflector with an opening angle $\theta_{m}$ (see figure 12.1). The depth of the spherical reflector $v m$ (from vertex to mouth) is more than a wavelength $\lambda$ (so $v m=R\left(1-\cos \theta_{m}\right.$ ) $>\lambda$ ). This will result in a focusing effect. For smaller depth $v m$, the focusing will be less severe and for $v m<\lambda / 4$ the focusing will not occur (only diffraction, see paragraph 7.4.3).

Based on the wave field approach using the Kirchhoff Integral, as described in Chapter 6 , in any field point the pressure of the single reflected wave can be calculated.

\section{Sphere:}

If the source $B$ is located in the centre $O$ of a sphere, the SPL at the focusing point can be calculated from (7.3c):

$$
\Delta L_{1 m}=20 \lg k\left(1-\cos \theta_{m}\right)
$$

where: $\Delta L_{1 m} \quad$ SPL at the focusing point, relative to the SPL at $1 \mathrm{~m}$ from the source.

$k \quad$ wavenumber: $k=\omega / c$ with $c=$ propagation velocity of sound $[\mathrm{m} / \mathrm{s}]$

$\theta_{m} \quad$ opening angle of the sphere segment $0<\theta_{m} \leq \pi$

For a hemisphere $\left(\theta_{m}=\frac{1}{2} \pi\right)$ this will be:

$$
\Delta L_{1 m}=20 \lg k
$$

The increase in the sound pressure level only depends on the opening angle and frequency and not on the radius of the (hemi)sphere. All energy radiated in the (hemi)sphere returns to the centre, independent from the radius.

Should the source position not correspond to the centre of the sphere segment, for source positions $x<0,85 \sqrt{\lambda R}$ (with $x$ = distance from the centre), the reduction $\Delta L_{f}$ of the SPL in the focusing point (12.3) compared to the situation with the source in the centre, can be approximated from (7.32b):

$$
\Delta L_{f} \approx 20 \lg \left(\cos \left(q \frac{x^{2}}{\lambda R}\right)\right)
$$

with $q=\pi / 2$ for $\theta_{m} \geq \pi / 2$ and $q=\theta_{m}$ for $\theta_{m}<\pi / 2$. 
Cylinder:

With source $B$ located in the centre $O$ of a cylinder segment with opening angle $\theta_{m}\left(\theta\right.$ ranging from $-\theta_{m}$ to $\left.\theta_{m}\right)$, the SPL at the focal point can be calculated from (8.9c):

$$
\Delta L_{1 m}=20 \lg \left(\frac{\theta_{m}}{\pi}\right)+10 \lg \left(\frac{\pi k}{R}\right)
$$

The length of the cylinder $l$ has to be $l>\sqrt{\lambda R}$.

In case of a full cylinder $\left(\theta_{m}=\pi\right)$ :

$$
\Delta L_{1 m}=10 \lg \left(\frac{\pi k}{R}\right)
$$

Contrary to a sphere, for a cylinder the $\Delta L_{1 m}$ is dependent on the radius of the cylinder, since there is a concentration in one direction and convergence in the other (along the axis of the cylinder).

Should the source position not correspond to the centre of the cylinder segment, for positions $x<0,75 \sqrt{\lambda R}$ (with $x=$ distance from the centre), the reduction $\Delta L_{f}$ of the SPL in the focusing point (12.6) compared to the situation with the source in the centre, can be approximated from $(8.28 \mathrm{~b})$ :

$$
\Delta L_{f} \approx 20 \lg \left(\cos \left(1,4 q \frac{x^{2}}{\lambda R}\right)\right)
$$

with $q=\pi / 2$ for $\theta_{m} \geq \pi / 2$ and $q=\theta_{m}$ for $\theta_{m}<\pi / 2$.

In case of an elliptic cylinder with elliptical axes $a$ and $b(a>b)$, the SPL in the focal point, with the source in the other focal point, can be derived from (8.10) and (8.11):

$$
\Delta L_{1 m}=10 \lg \left(\frac{b \pi k}{a^{2}}\right)
$$

\section{Ellipsoid:}

The SPL in the focal point of a prolate ellipsoid (with axis $a, b$ and $c$, for which $a=b$ and $c>a$, see also figure 9.1), with the source in the other focal point, may be obtained from the SPL in the focal point of a sphere (12.3) or (12.4) with a reduction factor $\Delta L_{f}$ of the SPL in the focal point compared to the SPL in the focal point of a sphere, obtained from (9.4):

$$
\Delta L_{f}=28 \lg \left(\frac{a}{c}\right)
$$

Figure 12.2 shows the $\Delta L_{1 m}$ at the focal point for a hemisphere and for cylinders with different radii. It shows the linear dependency on frequency for both shapes.

The $\Delta L_{1 m}$ of ellipsoids will be close to the line of the hemisphere, depending on the ration $a / c$, according to (12.10)

Figure 12.2 shows that the focusing effect of a spherical reflector, that is curved in two directions, is much stronger than the focusing effect of a cylinder, that is curved in only one direction. 


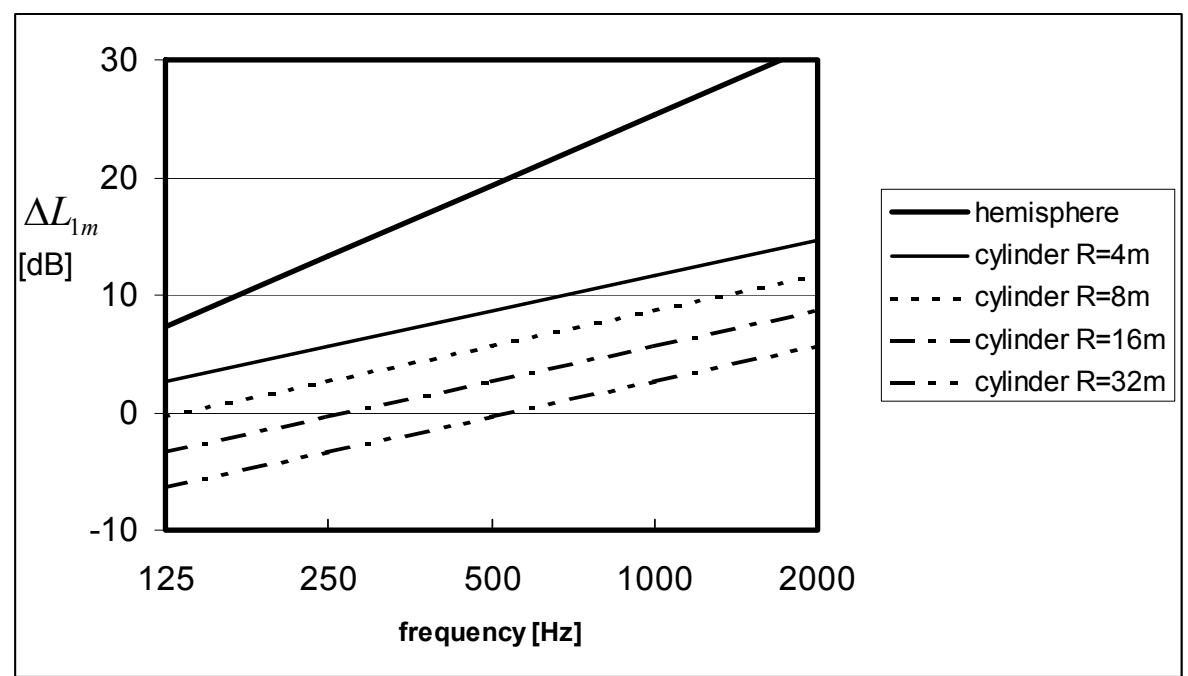

Figure 12.2. The $\Delta L_{1 m}$, the reflected $S P L$, relative to the $S P L$ at $1 \mathrm{~m}$ from the source, at the focal point or focal line for a hemisphere (12.4) and a cylinder with radius $R=4,8,16$ and 32 $m$ (12.7). The reflected SPL of the hemisphere is independent of the radius.

At the position of the receiver, the SPL of the reflection compared to the direct sound will be:

$$
\Delta L=\Delta L_{1 m}+20 \lg \left(r_{d}\right)
$$

To evaluate the strength of the reflection at a receiver position at distance $u$ from the reflecting surface, compared to the strength of reflections from a plane, fully reflecting surface, with the same traveled distance, the $\Delta L_{c}$ can be determined from:

$$
\Delta L_{c}=\Delta L_{1 m}+20 \lg (s+u)
$$

The method presented above will be illustrated in the cases described in chapter 14 . In most cases it will be sufficient to know the maximum pressure.

In the next two paragraphs the sound field in the focusing plane and along the specular line is described, for those situations that more information is needed on the distribution of the pressure within the focusing area. 


\subsubsection{Pressure in the focusing plane}

\section{Sphere:}

The focusing plane is the plane through the focusing point and normal to the specular axis of reflection. The pressure in this focusing plane will be high at the centre (focusing point), rapidly decreasing outside the centre, with some remaining side lobes due to interference (see figure 7.8).

Within the focusing area (see figure 12.1) the $\Delta L_{f}$, the pressure relative to the pressure in the focusing point can be obtained from (7.60):

$$
\Delta L_{f} \approx 20 \lg \left(\cos \left(\frac{x R k \sin \theta_{m}}{2 w}\right)\right)
$$

where $x$ is the distance to the focusing point in the focusing plane (see figure 12.1).

A calculation example is given in figure 12.3 for 3 frequencies. The practical meaning of this distribution function is that the area where the focusing effect can be noticed is much larger for the lower frequencies than for the higher ones. For example a receiver positioned at $0,3 \mathrm{~m}$ from the geometrical focusing point is outside the focusing area at $1000 \mathrm{~Hz}$, however for the $250 \mathrm{~Hz}$ signal this point is still within the focusing area. So the amplification will be strongly frequency-dependent. Since the area where the low frequency amplification occurs is much larger, focusing problems are mostly perceived as rather low or middle frequency problems.

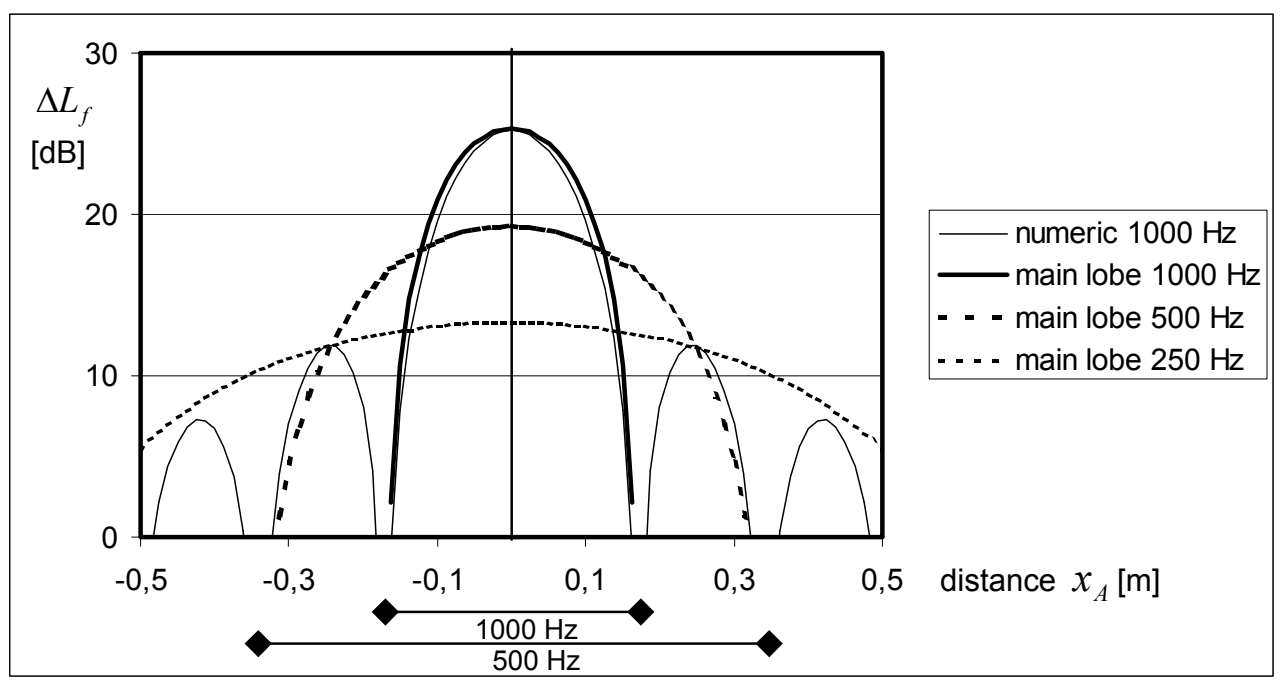

Figure 12.3. Calculation example of $\Delta L_{f}$, the $S P L$ in the focal plane of a hemisphere, relative to the SPL at $1 \mathrm{~m}$ from the source. Numerical solution (7.1) and the main lobe using (12.13) for $1000 \mathrm{~Hz}$. Also showing the main lobe for 500 and $250 \mathrm{~Hz}$. Under the graph: indication of the width of the focusing area for 500 and $1000 \mathrm{~Hz}$.

\section{Cylinder:}

When considering the radial plane of a full cylinder, for $|x|<0,35 \lambda$, the $\Delta L_{f}$, the SPL at distance $x$ from the centre (with the source in the centre) relative to the SPL in the focusing point, can be calculated from (8.31):

$$
\Delta L_{f} \approx 20 \lg \left(\cos \left(\frac{k x}{\sqrt{2}}\right)\right)
$$




\subsubsection{Pressure along the specular line}

The pressure at the specular line of reflection will be high at the focusing point and then decrease towards the edges of the focusing area.

Within the focusing area, at distance $z$ from the focusing point, the $\Delta L_{f}$, the SPL relative to the SPL in the focusing point can be approximated from (7.52):

$$
\Delta L_{f}=20 \lg \left\{\frac{R^{2}}{s(w+z)} \frac{\sin \left(\frac{1}{2} k R\left(1-\cos \theta_{m}\right)\left(\frac{R}{(w+z)}+\frac{2 z_{B}-R}{s}\right)\right)}{\frac{1}{2} k R\left(1-\cos \theta_{m}\right)\left(\frac{R}{(w+z)}+\frac{2 z_{B}-R}{s}\right)}\right\}
$$

Figure 12.4 illustrates this approximation in a calculation example. It shows that the approximation agrees quite well with the numeric solution. The geometrical solution is to be considered as an average of the oscillating reflected sound field and can be applied outside the points defined by (12.1).

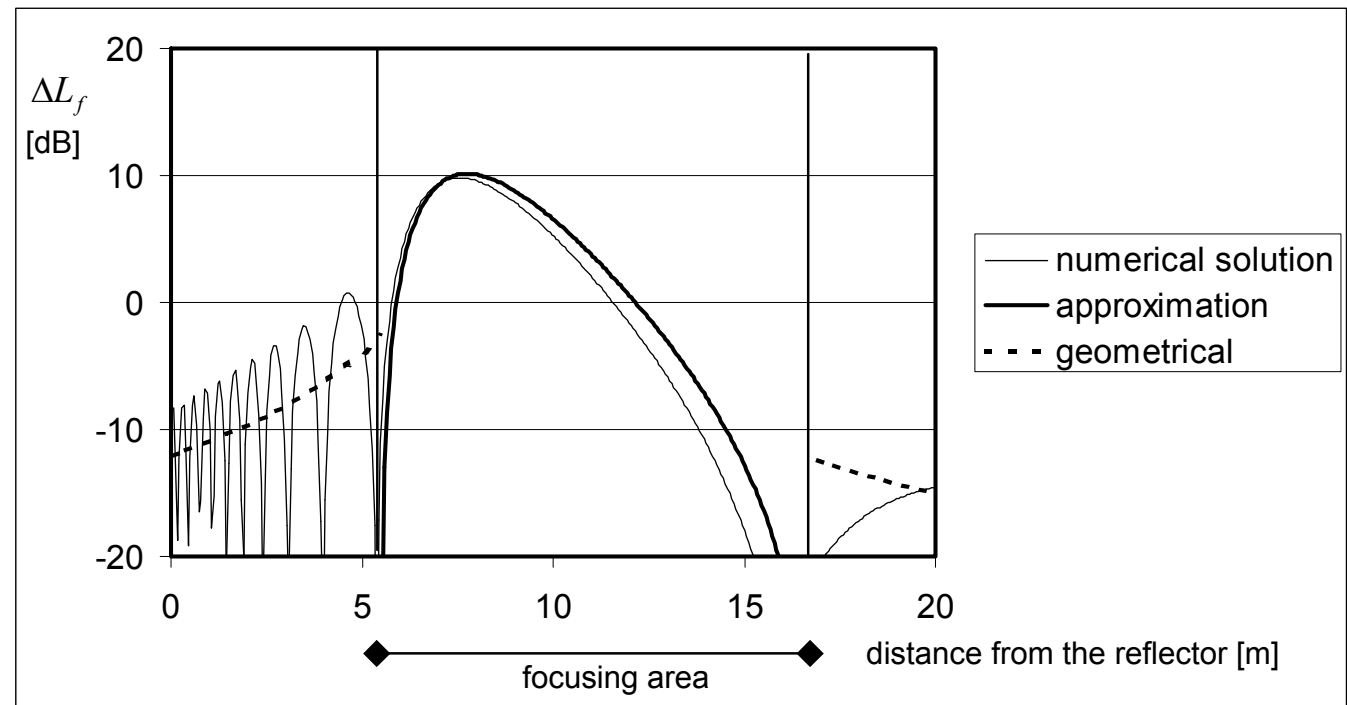

Figure 12.4. The $\Delta L_{f}$, the $S P L$ along the specular axis relative to the $S P L$ at $1 \mathrm{~m}$ from the source, in a calculation example: $R=5,4 \mathrm{~m}, f=1000 \mathrm{~Hz}, \theta_{m}=0,2 \pi, \theta=0, s=4 \mathrm{~m}(w=8,3 \mathrm{~m})$; numerical solution (7.1), approximation using (12.15) between the zeros given by (12.1) and the geometrical field by (5.10) outside the focusing area. 


\subsubsection{Calculation examples}

In this paragraph two examples will be given of the construction of the focusing area and the sound pressure within the focusing area.

Figure 12.5 shows the first calculation example of a hemisphere (opening angle $\pi / 2$ ) with a radius of $5,4 \mathrm{~m}$. The source is positioned outside the centre of the sphere segment, with $s=6,18 \mathrm{~m}$ and $\theta$ is $15^{\circ}$. From (5.6) the distance from the focusing point to the reflector can be calculated: $w=4,54 \mathrm{~m}$, so the position of the focusing point can be defined (see figure 12.5).
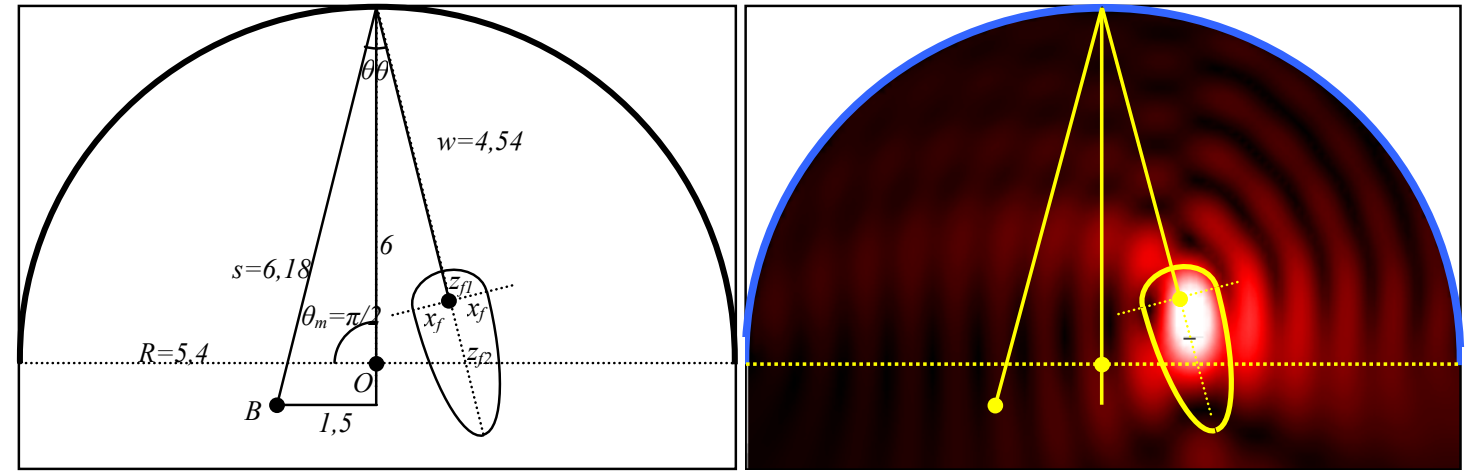

Figure 12.5. Calculation example of a hemisphere. Left: geometry with $B=$ source, focusing area for $250 \mathrm{~Hz}$. Right: numerical calculation result using (6.19), $250 \mathrm{~Hz}$. Source: $\hat{p}=1 \mathrm{~N} / \mathrm{m}$. Color range: white: $\geq 3 \mathrm{~Pa}$, black: $0 \mathrm{~Pa}$.

The next step will be to define the focusing area. For $250 \mathrm{~Hz}\left(k=4,6 \mathrm{~m}^{-1}\right)$ the size along the specular axis can be calculated with (12.1): $z_{f 1}=-0,45 \mathrm{~m}$ and $z_{f 2}=2,08 \mathrm{~m}$. The size in the focusing plane, from (12.2), will be $x_{f}=0,57 \mathrm{~m}$. The focusing area is indicated in figure 12.5 .

From (12.3) the pressure in the focusing point, with the source in the centre, can be calculated, this will be $\Delta L_{1 m}=13,3 \mathrm{~dB}$ (SPL relative to the SPL at $1 \mathrm{~m}$ from the source). Since the source is outside the centre, the SPL in the focusing point will be lower than with the source in the centre. From (12.5) the reduction is calculated: $\Delta L_{f}=-1,9 \mathrm{~dB}$. So the expected peak will be $\Delta L_{1 m}=11,4 \mathrm{~dB}$. Numerical calculations using (6.19) show a maximum of $10,9 \mathrm{~dB}$. The results of the numerical calculation are shown in figure 12.5. Figure 12.6 shows the SPL along the $z$-axis and the $x$-axis. Although there is a fair agreement, the results show that there is not an exact match between the approximation and the numerical result. Both the plots for the $x$-axis and the $z$-axis show a slight shift of the focusing point.
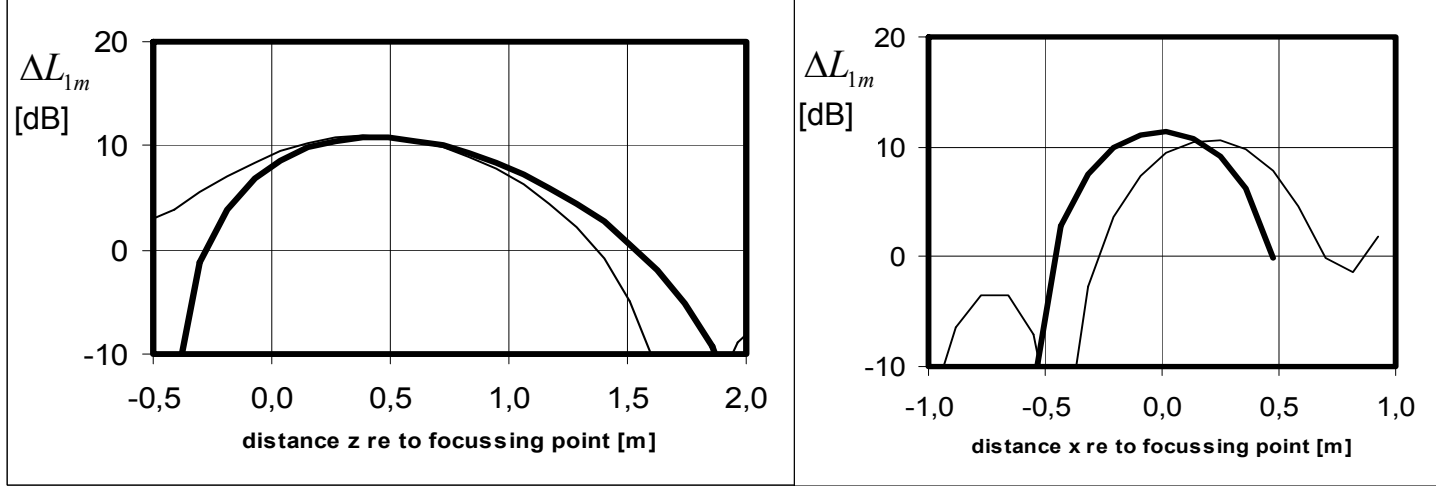

Figure 12.6. Calculation example shown in figure 12.5, SPL relative to SPL at $1 \mathrm{~m}$. Left: along the z-axis, Right: along the $x$-axis of the focusing area. Thin line: numerical calculation using (6.19); Thick line: approximation calculated using (12.3), (12.5), (12.13) and (12.15) 
The next example is indicated in figure 12.7. A sphere segment has an opening angle of $36^{\circ}(\pi / 5$ radians) and a radius of $5,4 \mathrm{~m}$. The depth of the segment $\mathrm{vm}$ is $1 \mathrm{~m}$, which is more than a wavelength for frequencies above $340 \mathrm{~Hz}$. The source is positioned outside the centre of the sphere segment, with $s=4,27 \mathrm{~m}$ and $\theta$ is $20^{\circ}$. From (5.6) the distance from the focusing point to the reflector can be calculated: $w=6,19$ $\mathrm{m}$, so the position of the focusing point can be marked (see figure 12.7).

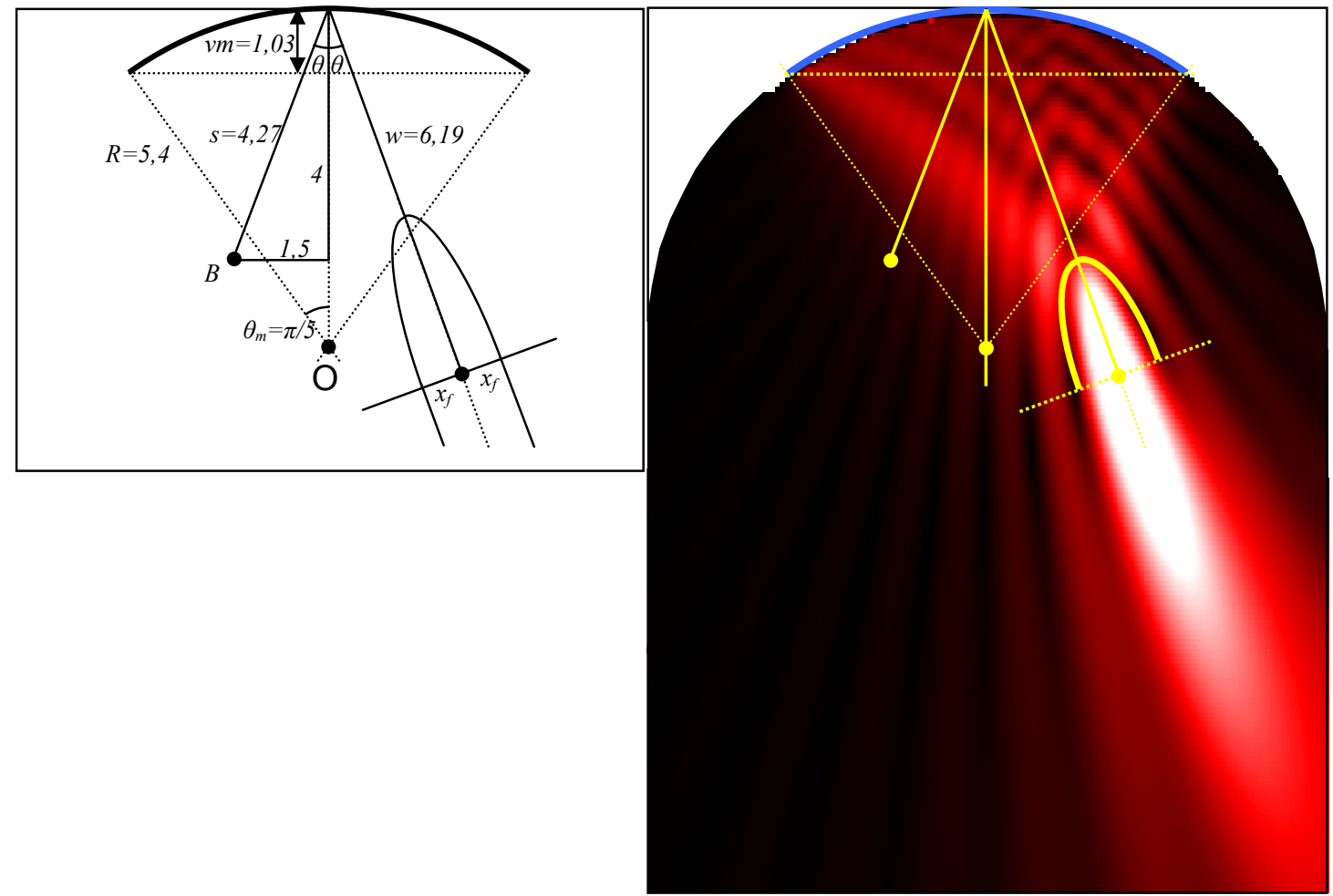

Figure 12.7. Calculation example of a sphere segment. Left: geometry with $B=$ source, focusing area for $500 \mathrm{~Hz}$. Right: numerical calculation result using (6.19). Source: $\hat{p}=1 \mathrm{~N} / \mathrm{m}$. Color range: white: $\geq 1 \mathrm{~Pa}$, black: $0 \mathrm{~Pa}$.

For $500 \mathrm{~Hz}\left(k=9,2 \mathrm{~m}^{-1}\right)$ the first $z_{f}$ of the focusing area along the specular axis can be calculated using (12.1): $z_{f 1}=-1,93 \mathrm{~m}$. The second zero does not exist, thus there is no interference behind the focus point. The half-width of the focusing area, from (12.2), will be $x_{f}=0,66 \mathrm{~m}$. The focusing area is indicated in figure 12.7, left.

From (12.3) the pressure in the focusing point, with the source in the centre, can be calculated, this will be $\Delta L_{1 m}=4,9 \mathrm{~dB}$ (SPL relative to the SPL at $1 \mathrm{~m}$ from the source). Since the source is outside the centre, the SPL in the focusing point will be lower than with the source in the centre. From (12.5) the reduction is calculated: $\Delta L_{f}=-3,4 \mathrm{~dB}$. However, this is an overestimate (see figure 7.5), since the distance from the source to the centre of the sphere segment is $1,07 \sqrt{\lambda R}$, this is more than $0,8 \sqrt{\lambda R}$, for which the approximation of (12.5) is valid.

The expected peak will be about $\Delta L_{1 m}=1,5 \mathrm{~dB}$. Numerical calculations with (6.19) show a maximum of $3,2 \mathrm{~dB}$, indicating that indeed the reduction due to the distance from the centre is overestimated.

The results of the numerical calculation are shown in figure 12.7,right. Figure 12.8 shows the SPL along the $z$-axis and the $x$-axis. 
Again there is a fair amount of agreement. Apart from the slight difference in the peak level, these results also show a slight shift of the focusing point.
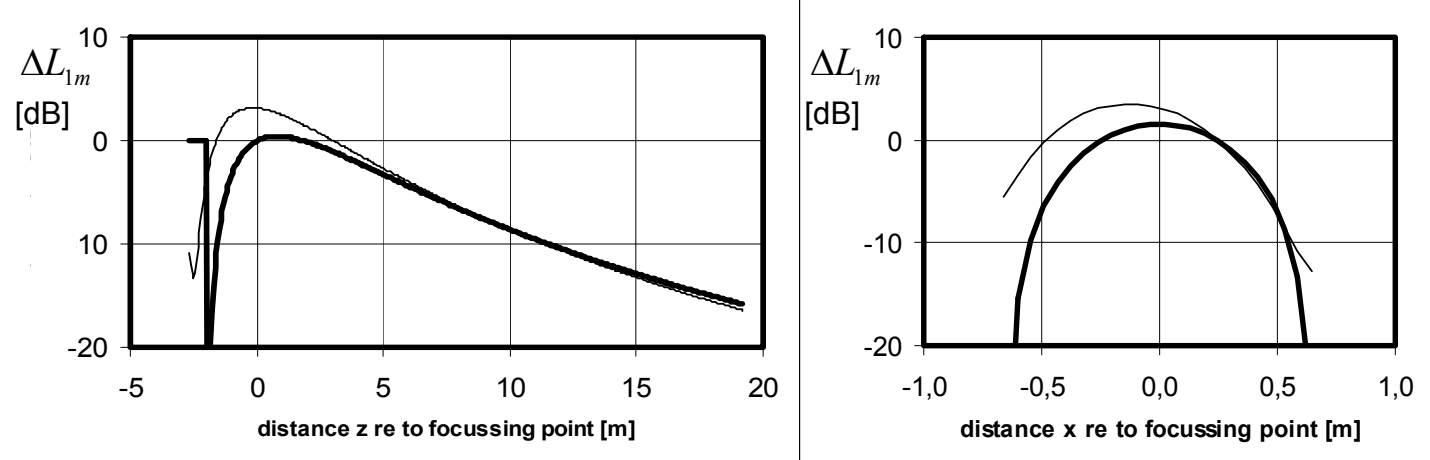

Figure 12.8. Calculation example shown in figure 12.7. Left: SPL along the z-axis, Right SPL along the $x$-axis of the focusing area. Thin line: numerical calculation using (6.19); Thick line: approximation calculated using (12.3), (12.5), (12.13) and (12.15)

It should be noted that for source positions either at the centre axis or at a distance $R$ from the reflector, the agreement within the focusing area is better (see e.g., figure 12.3 and 12.4). 


\subsection{Evaluation of the echo}

In this chapter methods are presented to calculate the SPL of the reflected sound from a curved surface. This calculation can be can either be obtained with the geometrical method described in paragraph 12.2 or the wave field method described in paragraph 12.3 .

There are basically two methods to evaluate the result in respect to echo perception. These methods will be summarized here, more details can be found in chapter 3 .

\section{Evaluation of the $\Delta L$}

The level difference $\Delta L$ is determined, this is the SPL of the reflection compared to the SPL of the direct sound at the receiver position, e.g. using (12.11).

This level difference $\Delta L$ may then be used for the evaluation of the reflection.

The maximum $\Delta L$ depends on the time delay $\Delta t$ between direct sound and reflection. If the actual $\Delta L$ is higher than this value, the reflection is likely to be audible as an echo.

For speech, the threshold of echo detectability can approximated, from (3.1), see also figure 3.5:

$$
\Delta L=-38,4 \lg (\Delta t)+55,1
$$

However, it should be noted that other reflections may mask the reflection from the curved surface to some extent (see also par. 3.6), so this requirement might be too stringent. The second approach using $\Delta L_{c}$ may be more appropriate.

\section{Evaluation of the $\Delta L_{c}$}

Instead of using (3.1) the excess sound pressure level over the other reflections from plane surfaces may be evaluated. Especially in a space with one curved surface and several plane surfaces, the reflection from the curved surface may be masked to some extent by the reflections of the plane surfaces. A direct way to evaluate this excess is by using $\Delta L_{c}$. The amplification $\Delta L_{c}$ is not only useful for obtaining the pressure in a receiver position, but it is also an indicator for the significance of the problem. The reflection from the curved surface will rise $\Delta L_{c} \mathrm{~dB}$ above other "normal" reflections with the same traveled distance. The reflection might be audible if the reflection from the curved surface arrives later than $50 \mathrm{~ms}$ after the direct sound $\left(u+s-r_{d}>17 \mathrm{~m}\right)$ and $\Delta L_{c}$ is larger than approx. 3-4 dB. This value is obtained from the method of Yamamoto in par. 3.4.

However this is just a first indication of possible echo disturbance. Single reflections from plane surfaces $\left(\Delta L_{c}=0\right)$, for example the rear wall of a theatre, may also result in echo perception.

This may especially be the case for directional sound sources.

The sound focusing by spherical or cylindrical shapes, as presented in par. 12.3.1 can be formulated in terms of $\Delta L_{c}$.

For source and receiver positions close to the centre of a sphere or a cylinder $(s+u \approx 2 R)$ the $\Delta L_{c}$ will be approximately (from 12.12):

$$
\Delta L_{c} \approx \Delta L_{1 m}+20 \lg (2 R)
$$

$\Delta L_{c}$ as a function of radius $R$ is given for the $500 \mathrm{~Hz}$ octave band in figure 12.9. 
Due to the SPL decrease of the reference (the SPL of the reflection from a plane surface, with the same travelled distance), the amplification $\Delta L_{c}$ increases with increasing radius. This is true both for the radius of the hemisphere and the cylinder.

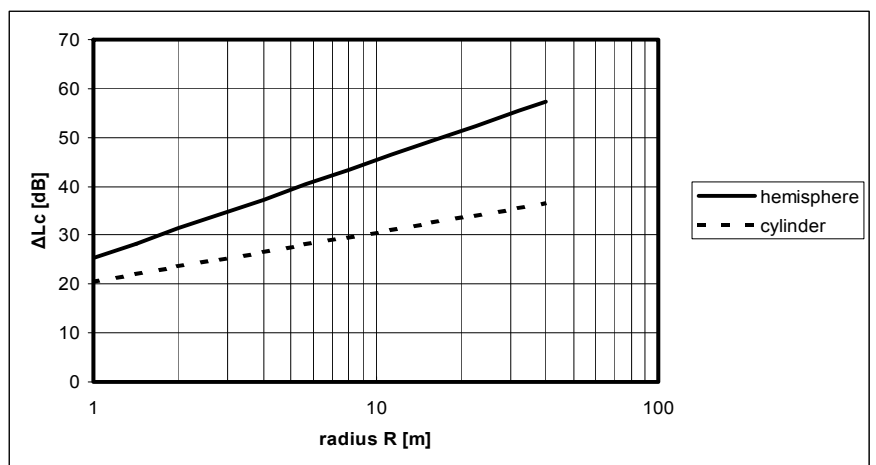

Figure 12.9. The SPL amplification $\Delta L_{c}$ at $500 \mathrm{~Hz}$ in a hemisphere, from (12.4) and (12.16), and in a cylinder, from (12.7) and (12.16), relative to the SPL of a reflection from a plane surface with the same travelled distance. Source and receiver are close to the centre.

With source and receiver close to the centre of the hemisphere or cylinder, the travelled distance of the reflection also depends on the radius. That means that the $x$-axis also represents the time delay of the reflection $(\Delta t=2 R / c)$

For a radius $R$ of more than $8,5 \mathrm{~m}$ the reflection will arrive after $50 \mathrm{~ms}$. For this size of hemisphere or cylinder the maximum amplification $\Delta L_{c}$ is more than $40 \mathrm{~dB}$ for the hemisphere and more than $30 \mathrm{~dB}$ for the cylinder. It is obvious that these reflections are heard as echoes.

For a radius $R$ of less than $8,5 \mathrm{~m}$ the reflection will arrive within $50 \mathrm{~ms}$. For source and receiver positions close to the centre, the amplification $\Delta L_{c}$ will still be more than $20 \mathrm{~dB}$ in this range. This will be perceived as strong coloration and may result in an inability to localise the source.

Both the geometrical method and the wave field method presented assume a fully reflective surface. Methods that can be used to reduce the sound pressure will be presented in the next chapter. 


\section{Chapter 13 \\ Reduction of reflected energy}

There are basically three methods of reducing the concentration of sound reflection. The first and most obvious one would be to alter the geometry of the curved surface in a way that its reflection would no longer be disturbing. The engineering approach presented in the previous chapter can be used in that design process.
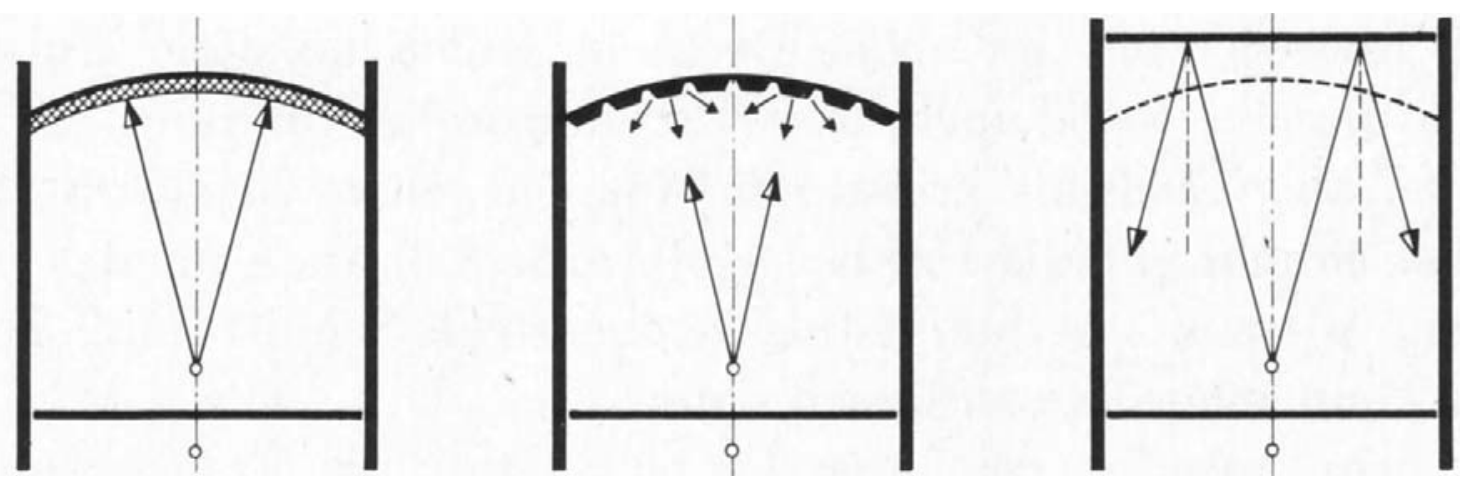

Figure 13.1. Three sound reduction principles for sound concentration: Left: sound absorption, middle: sound diffusion, right: transparent room boundary [Cremer78]

The other two basic methods of reducing the concentration of sound are diffusion and absorption. An additional possibility is to make a visual boundary in the room that is acoustically transparent. This way architecture and acoustics are separated. Behind the (curved) transparent boundary there might be a non-curved surface, or a surface that provides sound absorption or diffusion. These three methods are illustrated in figure 13.1.

In addition to these three methods, which are discussed in the next paragraphs, a method will be presented where the reflections are deliberately sent in a specific direction; this is named the redirection of reflections (see paragraph 13.4).

\subsection{Sound diffusion}

The focusing effect due to curved surfaces is caused by the convergence of energy and by the addition of pressure with an approximately equal phase in the focusing area. As shown in paragraph 7.5 and 8.4, randomizing the phase relation by using of diffusion takes away the focusing effect. So the application of diffusion may be an important option that can be used to reduce the focusing.

The SPL in the focusing point as indicated in figure 12.2 is the maximum SPL that can occur with a perfectly reflecting surface. Although the expected SPL at high frequencies is higher, problems most commonly arise for lower frequency bands, typically those around $500 \mathrm{~Hz}$. For high frequencies there is generally more diffusion and the focusing area is smaller.

The sound reduction in a focusing point by using a practical sound diffusing structure depends on the diffusing properties of the structure. It must be determined how much of the incident energy will be diffused and how much of the (remaining) energy is reflected in the specular direction.

Examples of some convex diffusers are shown in figure 13.2. 

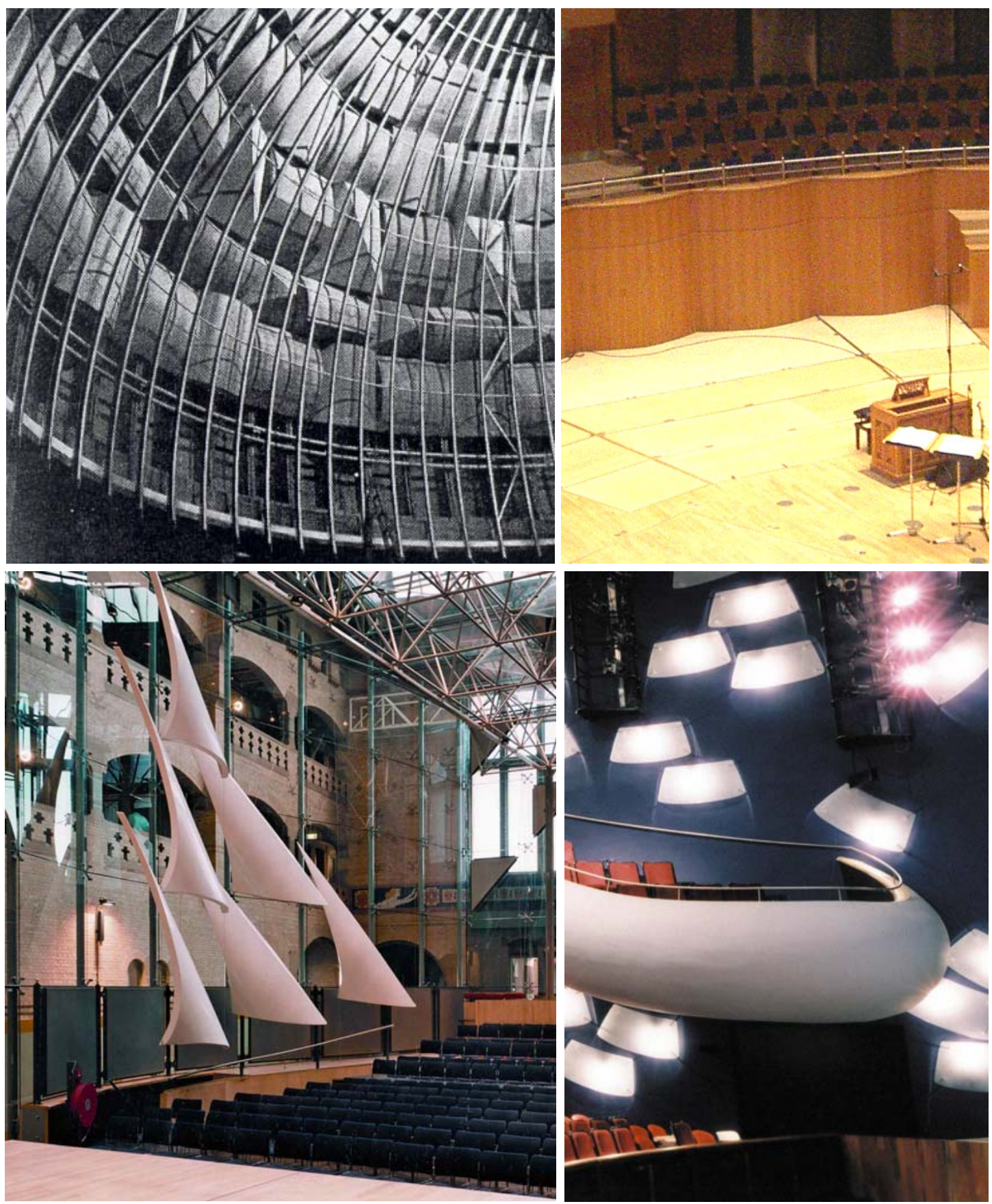

Figure 13.2. Examples of convex diffusing structures. Upper left: the diffusers in the dome of the former Nürnberger Planetarium [Cremer78]. Upper right: rear stage wall of the Tonhalle Düsseldorf; Lower left: convex sails in the glass recital hall inside the "Beurs" (Stock Exchange) building by architect Berlage, Amsterdam. Lower right: diffusion by convex car window panes in theatre De Harmonie, Leeuwarden. 
Diffusers do not necessarily have to be convex. Figure 13.3 shows some examples of other shapes applied in halls.

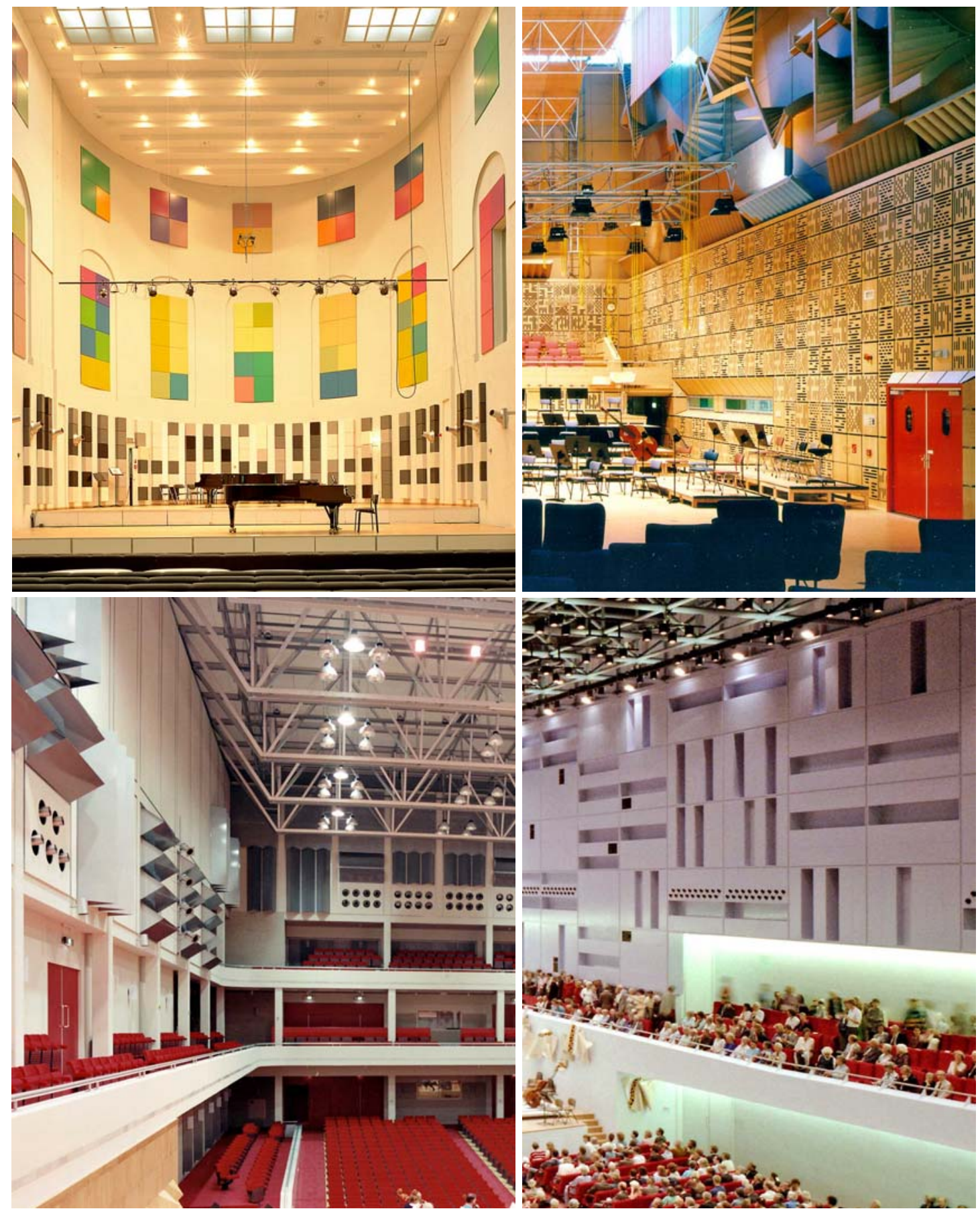

Figure 13.3. Examples of diffusing structures. Upper left: recital hall with half cylindrical stage in the School of the Arts (conservatory), Utrecht. Upper right: Rehearsal hall "Studio 5" of the MCO (Music foundation of Dutch radio and television), Hilversum, diffusion by "staircases" attached to the upper walls. Lower left: Concert hall Muziekcentrum, Enschede, Lower right: concert hall Dr. Anton Philipszaal, The Haque. 
It is also possible to diffuse sound using a Schroeder-diffuser, which has wells of different depths, see figure 13.4. The phase differences caused by the reflections from these different wells, causes a diffusing reflection pattern. The pattern of the wells may be based on different sequences, such as the quadratic residue sequence (see e.g., [Cox04]).

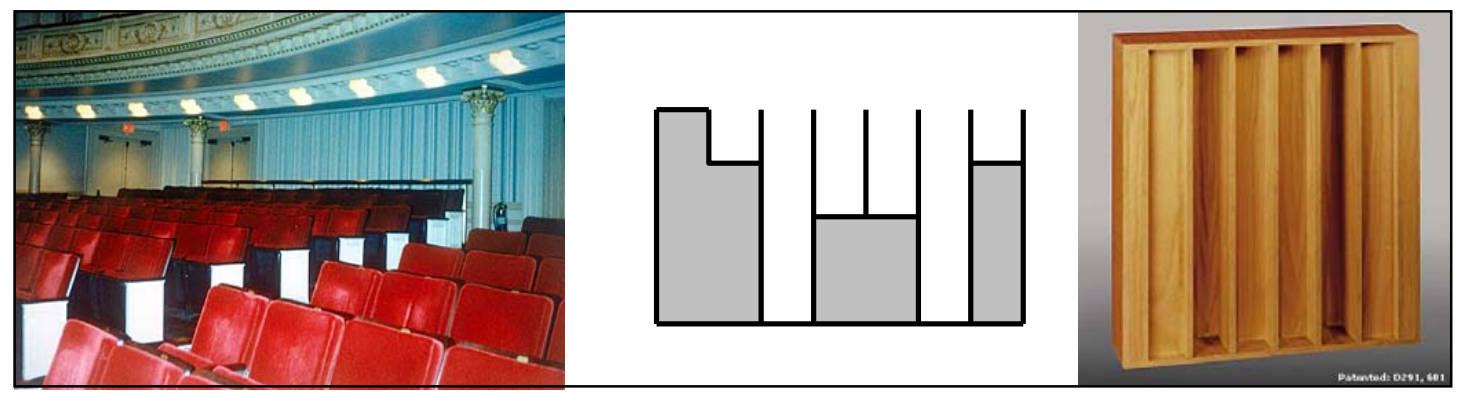

Figure 13.4. Example of a Schroeder diffuser. Left: Carnegie Hall, New York with rear wall covered with a quadratic residue diffuser with a depth of $15 \mathrm{~cm}$; Middle: section of a $N=7$ quadratic residue diffuser ; Right: Picture of wooden $N=7$ quadratic residue diffuser, from [Cox04] and Beranek[96].

The lower frequency of a Schroeder-diffuser is wavelength related. For a high diffusion, a well depth of $\lambda / 4$ is required (see par. 7.4.3). The efficiency of the diffuser however, not only depends on the single diffuser but also on the combination with the adjacent structure, e.g., other diffusers.

The diffusing properties of a material can be determined in a reverberation room according to [ISO17497-1] or in an anechoic room according to [AES01] (now in preparation as ISO17497-2). To investigate how much energy can be removed from the specular direction, measurements are taken of the angular dependent reflection properties of an array of half pipes.

$6,67 \mathrm{~m}$
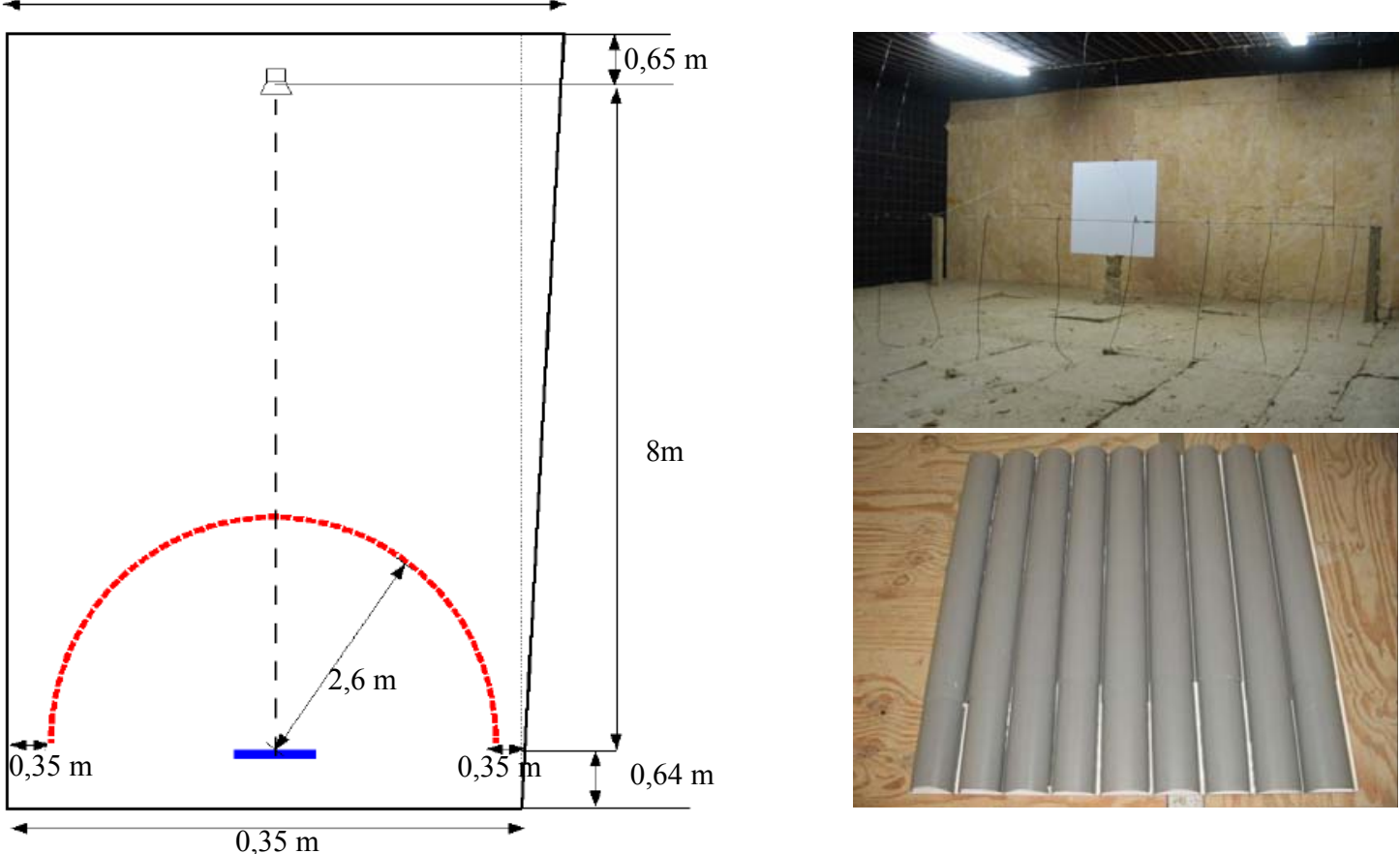

Figure 13.5. Measurement of the directional characteristics of the reflected sound according to [AES01]. Ground plan of the measurement setup(left), photo of the free field measurement facility with measurement setup (upper right) and array of half pipes measured, 9xø110mm (scale 1:12, so at 1:1 this will be $R=1,32 \mathrm{~m}$ ). Size of the measurement object $1 \times 1 \mathrm{~m}^{2}$. 
Figure 13.5 shows a measurement facility (Peutz' acoustics laboratory) for a free field method of measuring the directional properties of the reflection of small elements, e.g. scale models (see [AES01], [Hargreaves00]). Also shown is the array of half pipes being measured. Figure 13.6 shows some polar plots of the reflected energy from this array of half pipes. This figure shows that for this construction the reduction of specular sound is limited; there is still a significant amount of energy returned in the specular direction $\left(0^{\circ}\right)$.

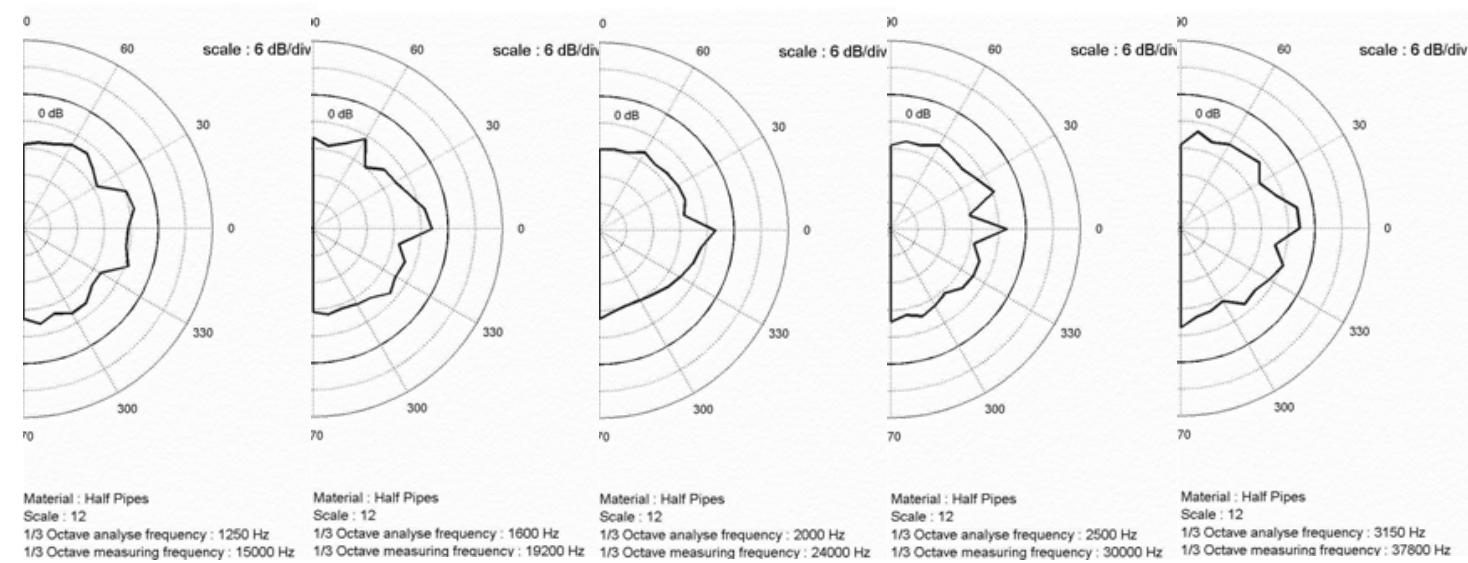

Figure 13.6. Polar plots of the measured reflection from an array of half pipes, frequencies $1250-3150 \mathrm{~Hz}$ (on scale 1:12; measuring frequencies 15000-37800 Hz).

The sound diffusing properties of a material can be described by the scattering coefficient, which describes the ratio between diffuse energy and specular reflected energy and is measured according to [ISO17497] (see also [Mommertz96], [Geetere04]).

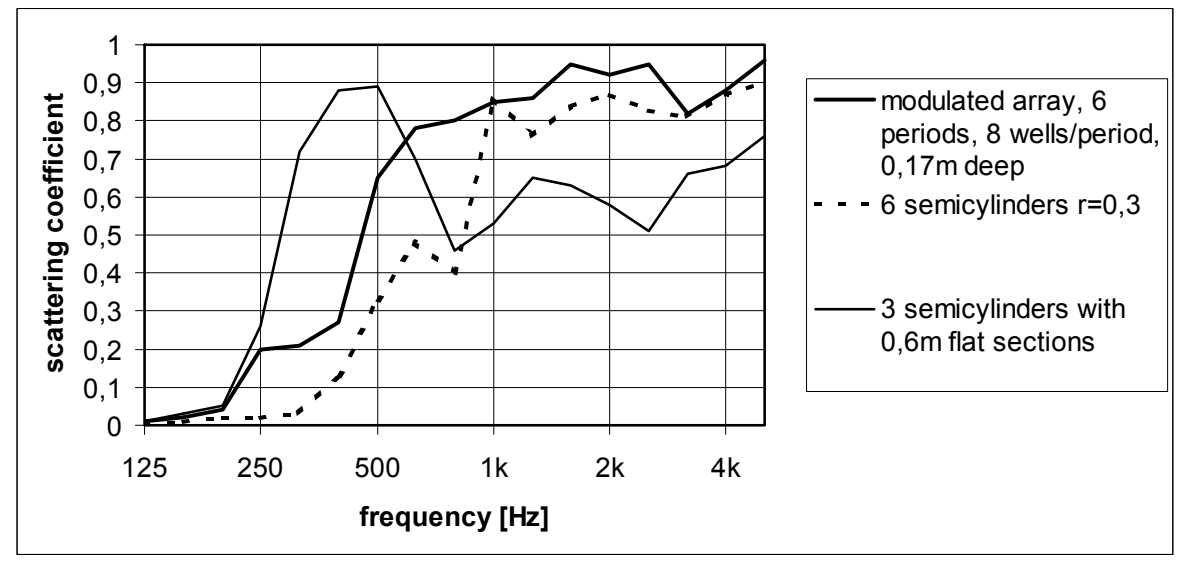

Figure 13.7. The scattering coefficient of three different diffusers (from [Cox04])

Figure 13.7 shows some typical data of the scattering coefficient of well diffusing structures (taken from [Cox04]). These data confirm the measurement results shown in figure 13.6. For practical diffusers, the reduction of specular energy that can be obtained is limited. The scattering coefficient is generally not more than $90 \%$, indicating a maximum of a $10 \mathrm{~dB}$ reduction of the specularly reflected energy. And if the $90 \%$ is achieved, than this is only for a limited frequency range. Especially at low frequencies a $10 \mathrm{~dB}$ reduction is difficult to obtain. 


\subsection{Sound absorption}

If it is the case that the reflected sound is reduced due to the absorption of the surface, the pressure of the reflected sound wave has to be multiplied by the (complex) reflection factor $R_{Z}$ that is related to the surface impedance $Z$ : ( $R_{Z}=\left|R_{Z}\right| e^{i \chi}$, with $0 \leq\left|R_{Z}\right| \leq 1$ ). Both the pressure and the velocity field in (6.18) will be reduced by the reflection factor. If the reflection factor is uniform over the surface $S:$

$$
\begin{aligned}
p_{A} & =\frac{\hat{p}}{4 \pi} \int_{S}\left(R_{Z} \frac{1+j k u}{u} \cos \varphi \frac{e^{-j k(s+u)}}{s u}+R_{Z} \frac{1+j k s}{s} \cos \alpha \frac{e^{-j k(s+u)}}{s u}\right) d S \\
& =R_{Z} \frac{\hat{p}}{4 \pi} \int_{S}\left(\frac{1+j k u}{u} \cos \varphi \frac{e^{-j k(s+u)}}{s u}+\frac{1+j k s}{s} \cos \alpha \frac{e^{-j k(s+u)}}{s u}\right) d S
\end{aligned}
$$

In (13.1) it is assumed that the reflection factor is not dependent on the angle of incidence or the angle of reflection. In general, this is not a correct assumption. However, for a sphere with the source and receiver close to the centre, the assumption may be sufficiently valid. The reflection factor will be the reflection factor for normal incidence.

Using this formulation of the Kirchhoff integral, the pressure in the focusing point of a sphere segment (see (7.3b)) will become:

$$
|p(0, \omega)|=\left|R_{z}\right| k \hat{p}\left(1-\cos \theta_{m}\right)
$$

and the SPL relative to the pressure $1 \mathrm{~m}$ from the source (see (12.3)):

$$
\Delta L_{1 m}=10 \lg \left|R_{z}\right|^{2} k^{2}\left(1-\cos \theta_{m}\right)^{2}
$$

The relation of the reflection factor with the absorption coefficient (see e.g., [Kuttruff73]) is:

$$
\alpha=1-\left|R_{z}^{2}\right|
$$

so the SPL in the focusing point will be reduced due to absorption by:

$$
\Delta L_{1 m}=10 \lg (1-\alpha) k^{2}\left(1-\cos \theta_{m}\right)^{2}=10 \lg k^{2}\left(1-\cos \theta_{m}\right)^{2}+10 \lg (1-\alpha)
$$

The absorption of materials is wavelength-dependent. Some examples of absorption coefficients for random incidence measured in a reverberation chamber are given in figure 13.8 .

For porous absorbers a high absorption coefficient can be obtained if the thickness is more than $\lambda / 4$ or when the absorption material is placed at an air gap with this thickness. That means that to obtain high absorption at low frequencies a lot of space is required. An alternative is to apply a plate resonator type of absorber. At the resonant frequency a high absorption will be realised, but generally the absorption for higher frequencies is much less. By using highly damped resonators in combination with high frequency porous absorption, multi-layer systems can be created that provide high absorption over a wide frequency range. Figure 13.9 shows this optimised absorption applied to the slightly curved roof of the Rotterdam Ahoy Sportpaleis, a large venue for popular music. 


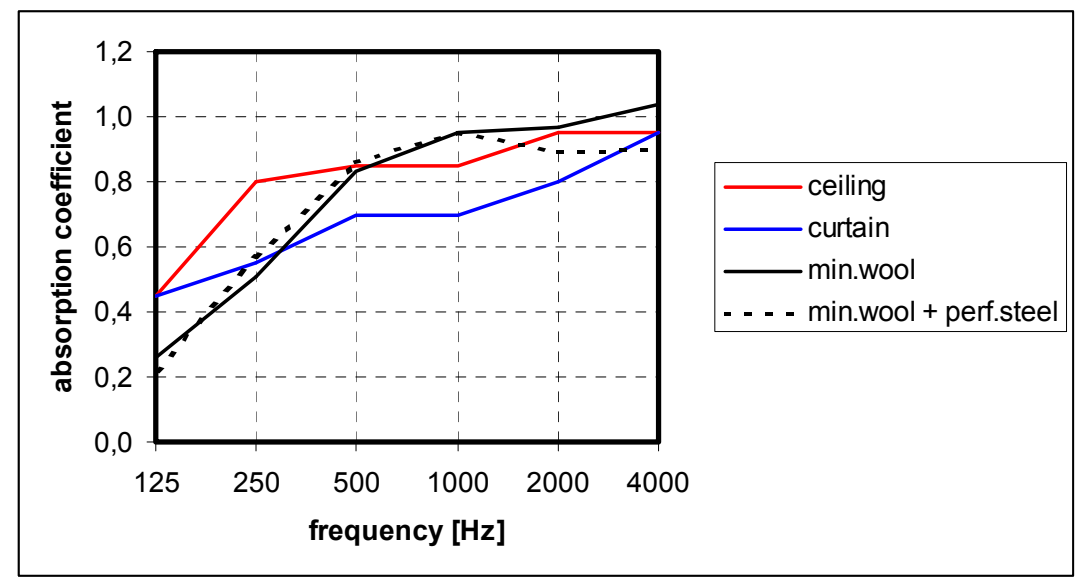

Figure 13.8. Some examples of absorption data, absorption coefficient measured in the reverberation chamber according to [ISO 354]. Ceiling: mineral wool tiles with an air gap of $200 \mathrm{~mm}$. Curtain: heavy curtain $0.5 \mathrm{~kg} / \mathrm{m} 2$, folded (factor 2) at $100 \mathrm{~mm}$ from the wall (on average). Min.wool: $50 \mathrm{~mm}$ of mineral wool, directly on the floor. Min.wool + perf. steel: 50 $\mathrm{mm}$ mineral wool with perforated steel on top, $29 \%$ open. Measurement data from the Peutz laboratory.

Due to the large dimensions and the resulting time delays, all the reflections in such a hall may cause disturbing echoes and thus the reflection strength had to be reduced by about $10 \mathrm{~dB}$. This requires a broad band absorption of $90 \%$ or more and could be realised for frequencies of $125 \mathrm{~Hz}$ and higher.
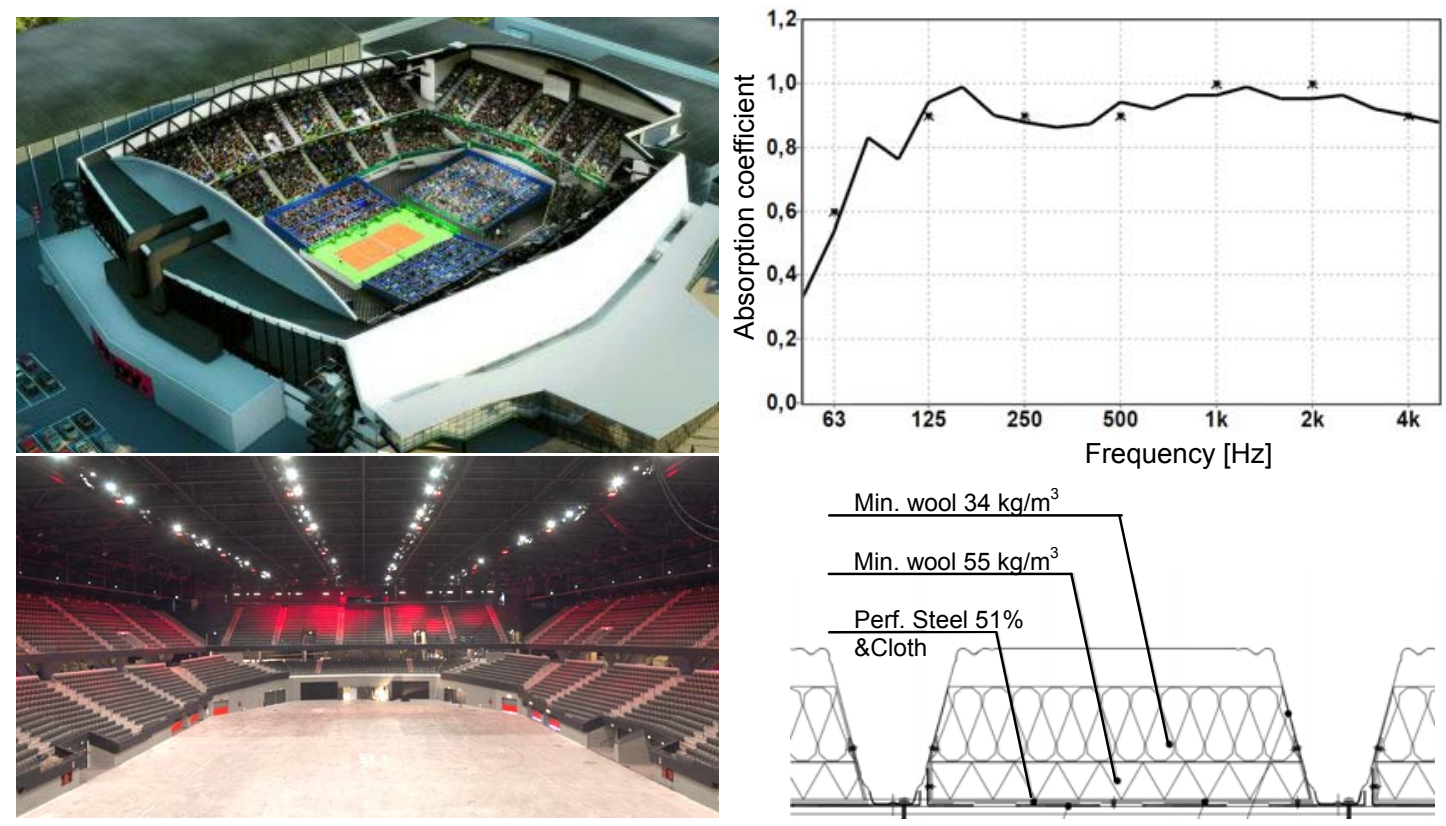

Figure 13.9. Sound absorption applied for the new curved roof of the enlarged Sportpaleis Ahoy in Rotterdam. Upper left: Artist impression of the enlarged venue (without roof), Lower left: Inside view after enlargement in 2010. Lower right: sound absorbing light weight roof construction, with the bottom side exposed. Upper right: Measured sound absorption coefficient according to [ISO354] in the Peutz laboratory, lines: third octave band results, asterisk: octave band results. 
It should be noted that the measurement of the absorption coefficient in the reverberation chamber suffers from inaccuracies, causing the $\left|R_{z}\right|^{2}=(1-\alpha)$ to be rather unreliable for high absorption coefficients. A more reliable method of obtaining the reflection coefficient, at least for normal incidence, would be the measurement with the impedance tube ([ISO10534-1] or [ISO10534-2]). The impedance tube measurement requires rather small samples (smaller than the wavelength) so it is only suitable for locally reacting materials.

A third method of measuring the reflection is a reflection measurement in an anechoic room based on [AES01], see also the next paragraph.

Since for a propagating sound wave the impedance of air in an absorbing material will always be different from the impedance in free moving air, the transition into the material will always cause some degree of reflection. A further improvement in absorption can therefore be obtained if the sound wave gradually enters the material, e.g., by application of wedges. In anechoic rooms very efficient sound-absorbing wedges with a length of about $1 \mathrm{~m}$ are usually needed to obtain the required absorption coefficient of $99 \%$ [ISO3745] at $100 \mathrm{~Hz}$, corresponding to an SPL reduction of $20 \mathrm{~dB}$.

It can be concluded that the data presented illustrate that, for practical applications, the maximum absorption that can be obtained is around 90 to $95 \%$. The reduction in reflected sound energy that can be obtained is therefore limited to 10 to $15 \mathrm{~dB}$. Especially for the low frequencies, it is very difficult to obtain reductions of $10 \mathrm{~dB}$ or more.

Figure 12.2 shows that the amplification by curved surfaces, especially those that are spherically-curved, can be (much) more than 10 or $15 \mathrm{~dB}$. That means that the application of absorption may be not sufficient for spherically-curved structures.

For cylindrical shapes however, the SPL in the focal line is much lower (see figure 12.2) and application of absorption or diffusing elements might be sufficient to take away the echo effects. Examples of sound absorbing structures in cylindrical halls and rooms are shown in figures 13.10 to 13.12 .

It is noted that a choice between absorption or diffusion or the combination of these methods also depends on other aspects like the reverberation time to be achieved. 

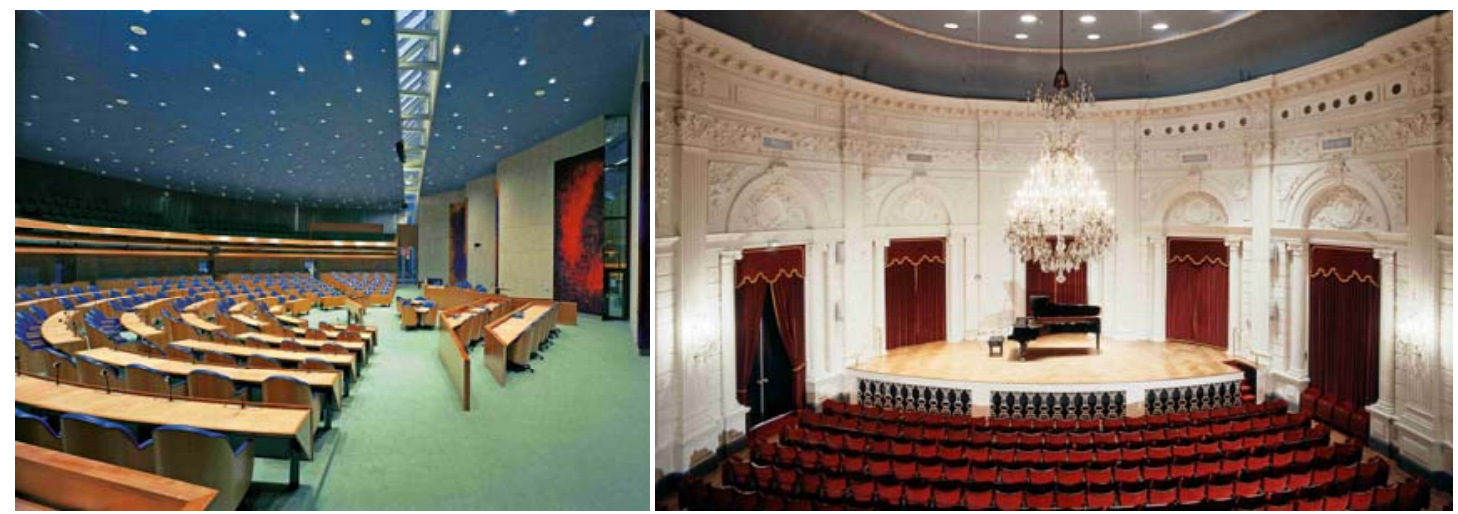

Figure 13.10. Examples of cylindrical halls with absorption: Left Tweede Kamer The Hague. The cylindrical rear wall is made highly absorptive with mineral wool and a covering of a wire mesh. Right: recital hall (kleine zaal) of the Concertgebouw, Amsterdam an elliptical cylinder where the focusing is reduced by a combination of curtains and diffusion.

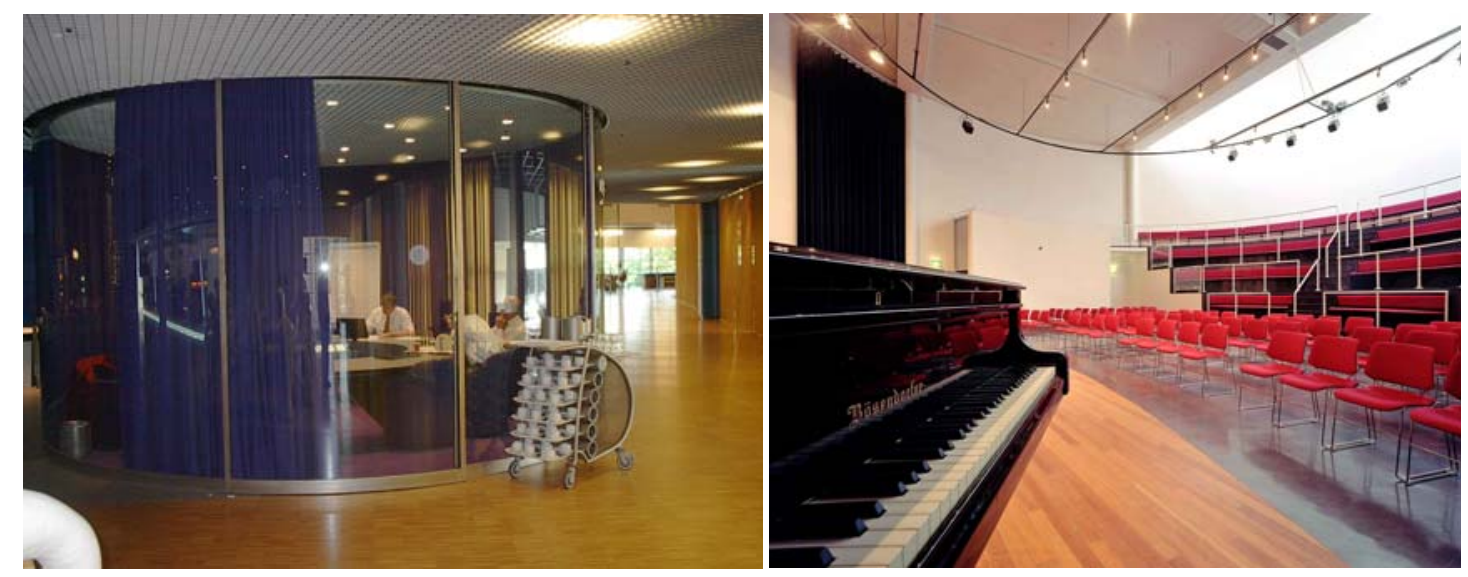

Figure 13.11. Examples of cylindrical rooms with curtains as (variable) absorption. Left: meeting room Interpolis in Tilburg, Right: recital hall (Keuningzaal) in the Centre for Arts and Culture ICO in Assen.
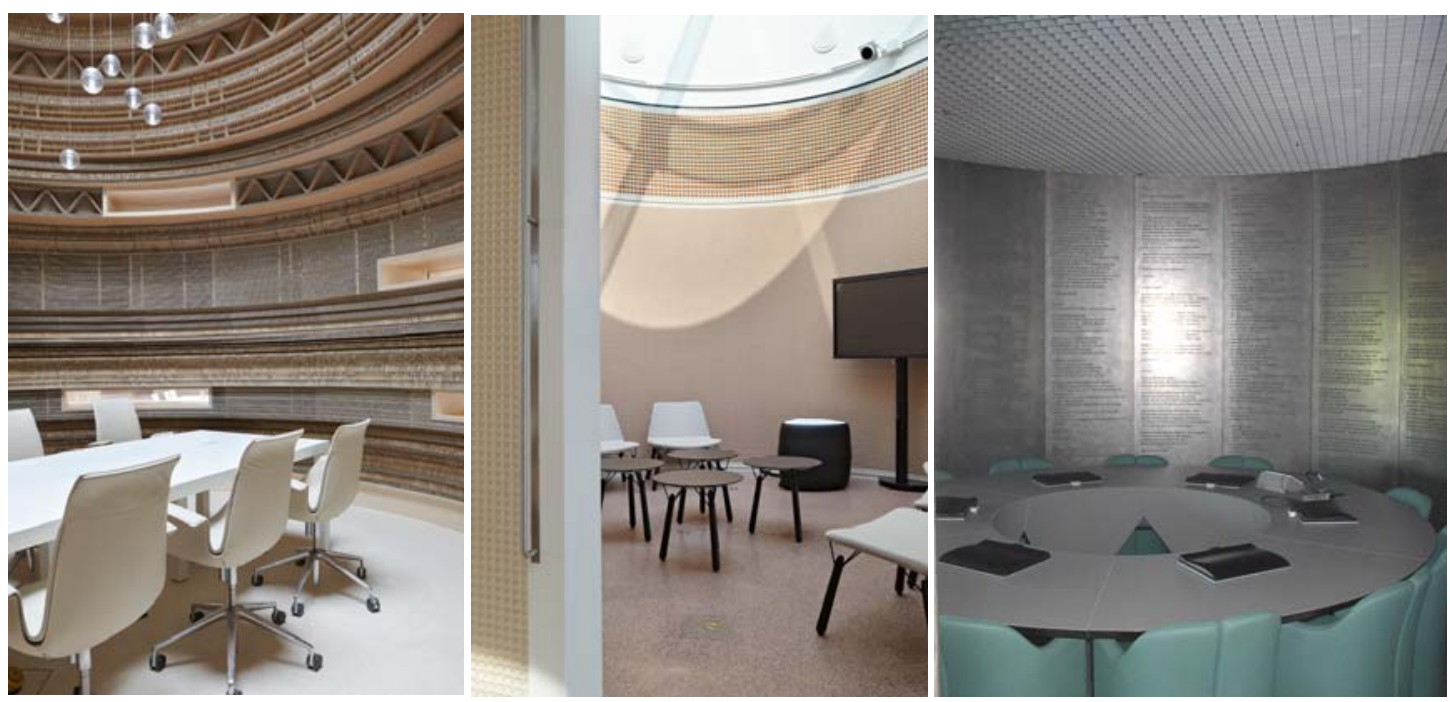

Figure 13.12. Examples of sound absorbing solutions in cylindrical meeting rooms. Left: Absorbing and diffusing structures of Cardboard. Middle: perforated structure in front of mineral wool. Right: letters as perforation in a steel plate with mineral wool behind it. Left and middle: Rabo, Utrecht, right: Interpolis, Tilburg 


\subsection{Acoustically transparent boundary}

An acoustically transparent boundary makes it possible to give the space an architectural shape, but have a different shape behind it. Examples of such structures are e.g., mentioned in [Sabine22] (Philadelphia planetarium) and Cremer[78] (Nürnberger Planetarium, figure 13.2 upper left).

Planetaria and space theatres are typically spaces that are hemispheres by definition. Since projection can be made onto a perforated construction, the diffusion or absorption can be solved behind that layer. See also the examples in figure 13.13.
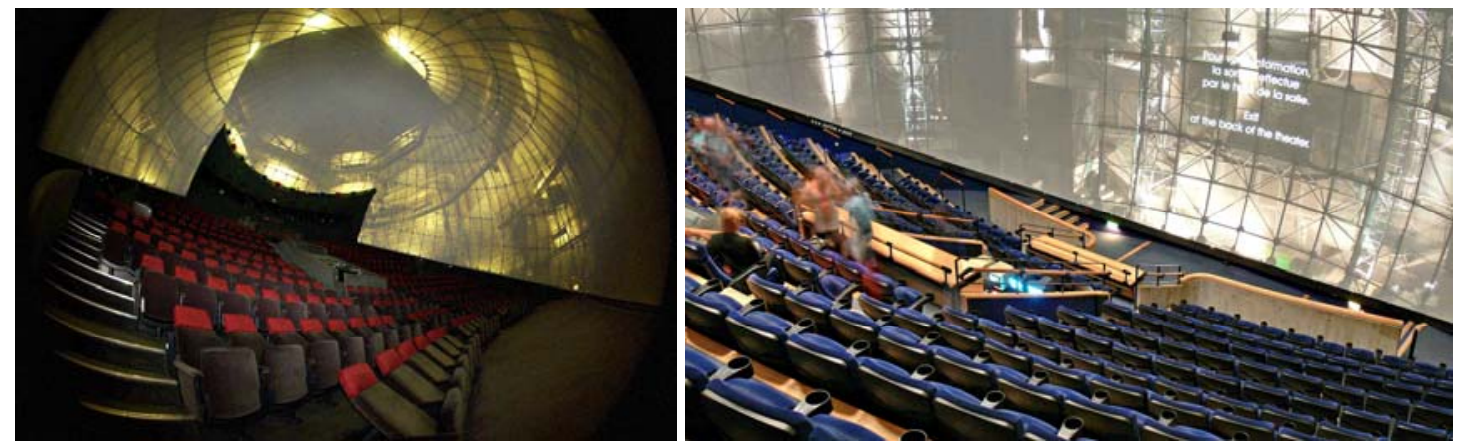

Figure 13.13. Examples of surround movie theaters. Left: Omniversum in The Hague, Right: La Geode at Parc de la Villette in Paris. Highly absorptive and diffusive materials were applied behind an acoustically transparent construction.

Another example of an acoustically transparent boundary is the renovated Tonhalle Düsseldorf, that is described more in detail in par. 14.3.

The transparency of the layer has to be sufficient. If this transparency is limited there will still be a significant amount of remaining (specular) reflection and the reduction of the focusing will be limited; this is comparable to the situation with a diffusing or an absorbing structure as described above. Perforated metal sheet or perforated wood panels are examples of materials with limited transparency, especially in the high frequencies. For the renovation of the Tonhalle Düsseldorf the transparency was investigated of a metal wire mesh with an open area of about $70 \%$ that was placed in the position of the former reflective dome [Lorenz06]. Including the reflections of the suspension frame, a $20 \mathrm{~dB}$ reduction of the reflected sound was achieved (see figure 13.14), which was sufficient to completely remove the echo, caused by this part of the cupola construction.
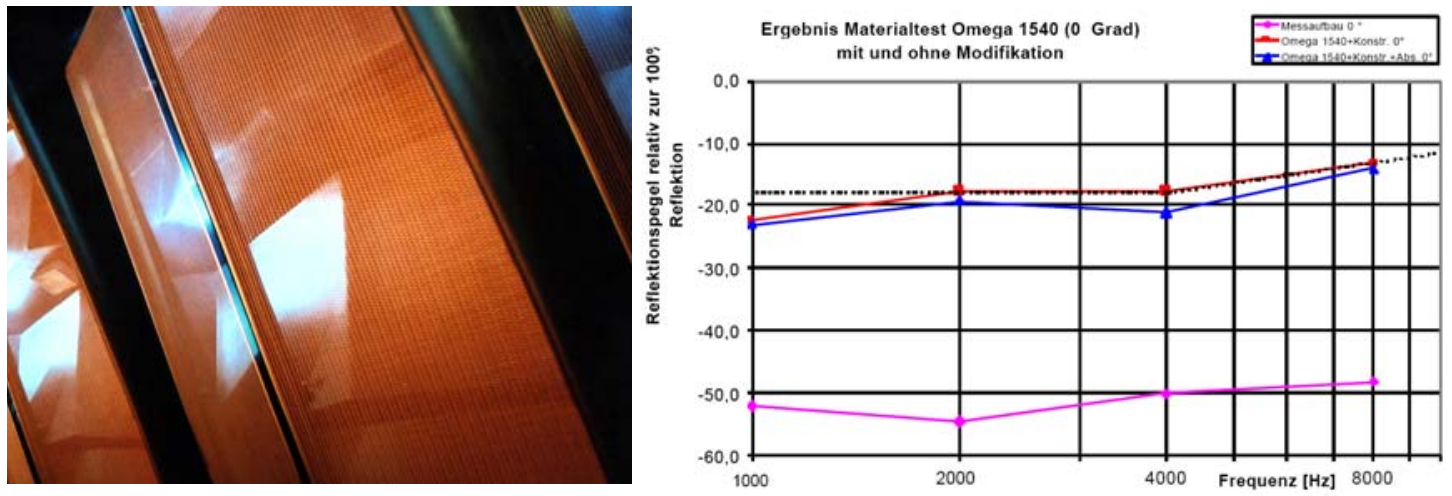

Figure 13.14. Left: photo of the wire mesh applied in the Tonhalle Düsseldorf. Right: Reflected energy in specular direction $\left(0^{\circ}\right)$ by a metal wire mesh (wire $1.5 \mathrm{~mm}$, distance 4 $\mathrm{mm}$ ), size $1 \times 1 \mathrm{~m}^{2}$, mounted on a steel frame, measured in an anechoic room (figure 13.2). Red line: with steel frame; Blue line: steel frame covered with absorption material; Purple line: reflected enerav without sample [Lorenz06] 


\subsection{Redirection of reflections}

If it is the case that the reflected energy can not be reduced sufficiently by using absorption or diffusion (which is generally the case if a reduction of $10 \mathrm{~dB}$ or more is required), then the reflected energy may be redirected, for example to areas where the focusing is not disturbing.

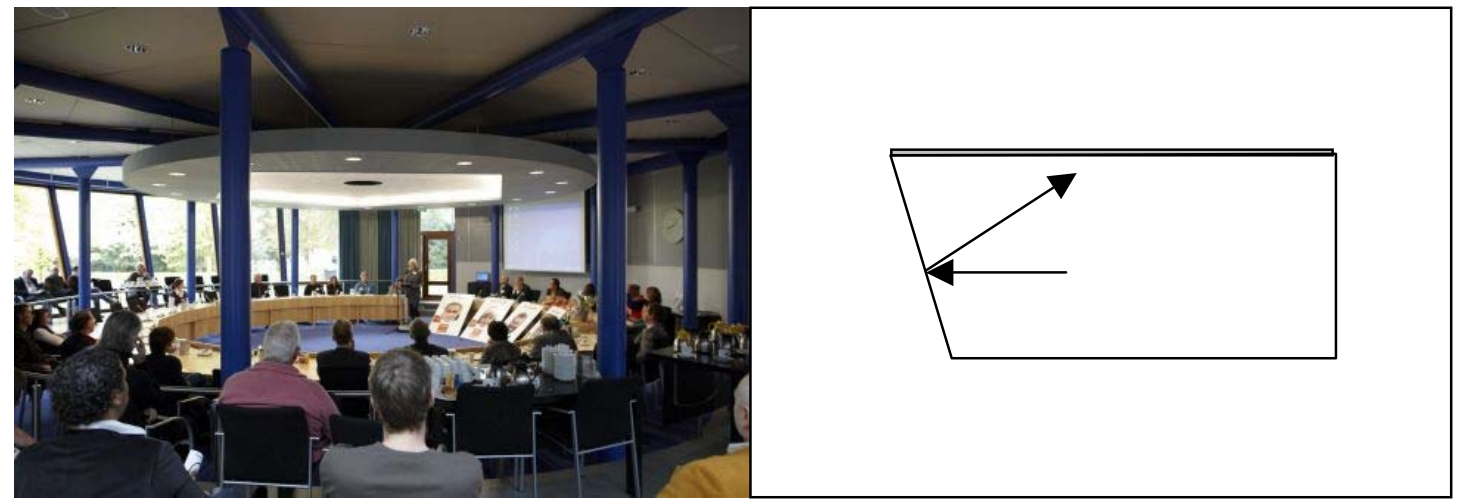

Figure 13.15. Example of a (half) circular town hall in Tiel. The circular glass facade is tilted (making it a cone segment). Left: picture of the interior. Right: schematic section showing the reflection redirected into the absorptive ceiling.

Figure 13.15 shows the section of a (half) circular town hall room where the concentrating reflection was redirected towards the (strong) absorbing ceiling.

When redirecting reflections by tilted panels, the size of the reflecting element is essential. If the size is too small, energy will still be reflected (diffraction) and add up at the focusing point. Figure 13.16 shows the reflected energy (reflected back to the source; both source and receiver in the far field) from a single rectangle flat panel, placed at an angle. The results show that the reduction is a function of panel size (related to wavelength) and angle of the panel. For example, with an angle of 30 degrees and a panel size of 2 times the wavelength, the reduction that can be obtained is approximately 15 to $20 \mathrm{~dB}$.

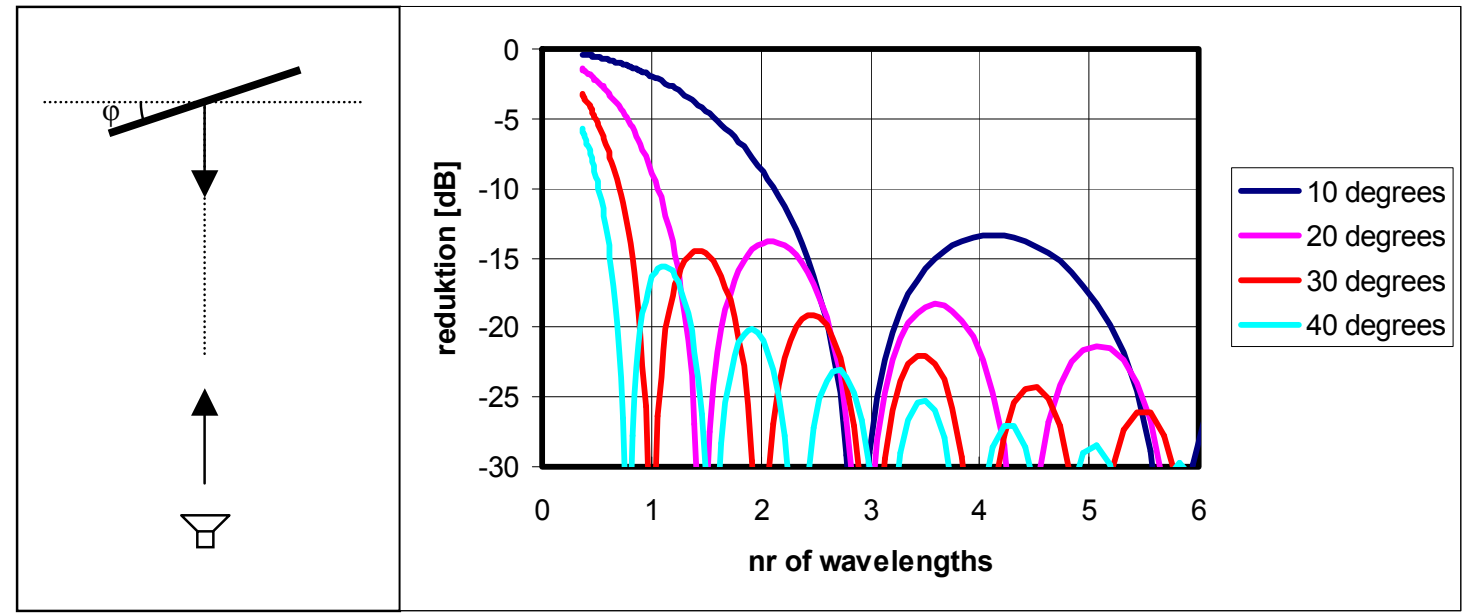

Figure 13.16. Reflected energy of a rectangle plane surface depending on the angle $\varphi$ and the size of the panel. Left: geometry of the situation; Right: X-axis: ratio of panel size to wavelengths, Y-axis: reduction of the reflected sound relative to the panel at angle of 0 degrees. Source and receiver are both in the far field. Calculated using (6.19). 
By combining tilted panels, prism-shaped reflectors can be made. Figure 13.17 shows how this prism works. With an angle of 30 degrees the energy will be reflected at 60 degrees. This is the maximum angle; a larger angle would result in double reflection.

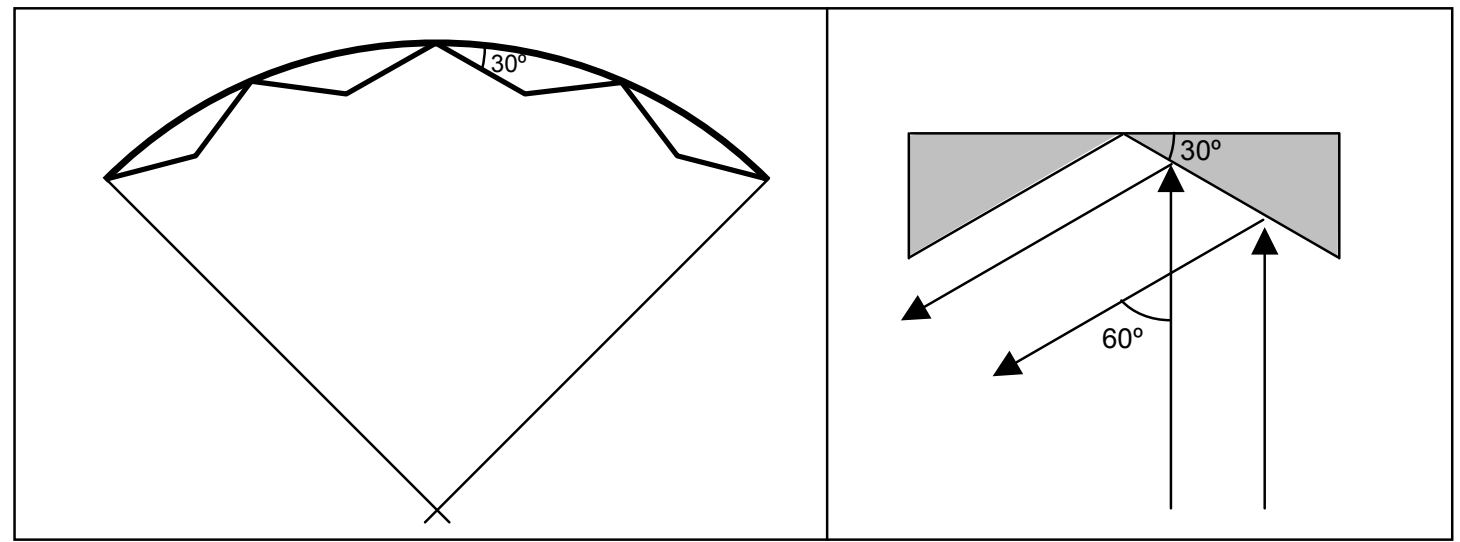

Figure 13.17. Redirection with prisms. Left: circular or sphere segment covered with $30^{\circ}$ prisms; right: reflection from a $30^{\circ}$ prism.

Measurements have been performed in an anechoic room according to [AES01] on a $1: 12$ scale model to verify the suppression of the specular reflection. The measured reflection of an array of twelve $30^{\circ}$ prisms is shown in figure 13.18 .

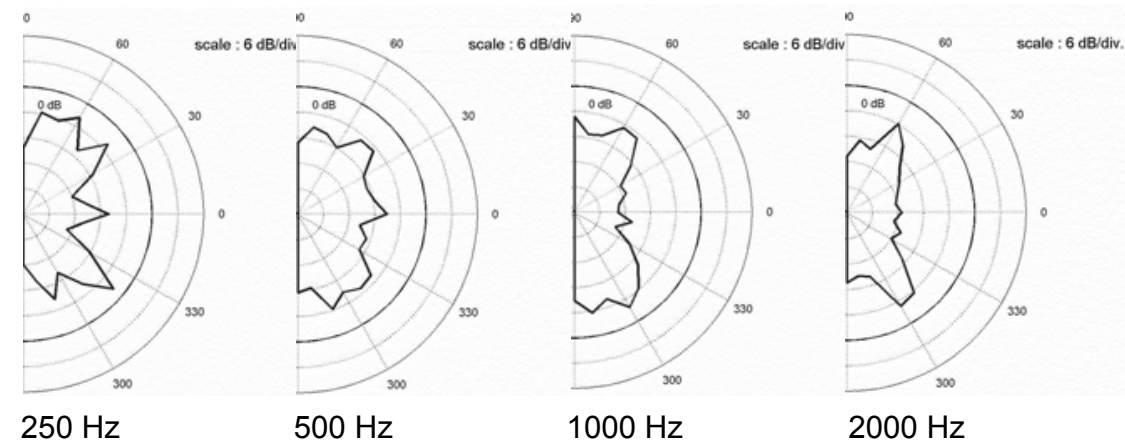

Figure 13.18. Measurements of the redirection of reflected sound by an array of $30^{\circ}$ prisms. Polar plots of the reflected sound pressure. Incident sound is at $0^{\circ}$. Measurement in an anechoic room according to [AES01]. Scale factor 1:12.

The width of the single panels at $30^{\circ}$ is $1,15 \mathrm{~m}$ (real scale). For a frequency of 600 $\mathrm{Hz}$ this size corresponds with two wavelengths. The results show that above this frequency there is a good suppression of the specular reflection. The maximum suppression that could be measured with this setup was approximately $18 \mathrm{~dB}$. This suppression is reached for the higher frequencies. 


\section{Chapter 14 \\ Cases of sound concentration}

In this chapter some cases of sound concentration will be described. Since the phenomenon speaks to the imagination, several cases of echoes have been described in literature. In paragraph $14.1 \mathrm{a}$ few of them will be discussed.

Some cases are evaluated using geometrical computer models (e.g., [Stephenson94]), which has a limited value, see chapter 5.

If a more exact approach is not adequate as described in chapter 12, e.g., because of the complexity of the geometry, the only valid way to evaluate sound concentration is to use scale model research. In paragraph 14.2 we will briefly describe the relevant issues for scale model research. This will be a useful introduction because the scale model research method is applied in the cases described in paragraph 14.3 and 14.4. In these cases, which were both concert halls that were notorious for their echo, extensive scale model research was done to solve these echo problems that were caused by spherical and ellipsoidal curved surfaces.

Paragraph 14.5 describes a special case, de "Klankkaatser", an ellipsoidal shaped space where music is created and can be heard publicly.

Paragraph 14.6 and 14.7 describe two cases in office buildings, the first with a cylindrical outer façade and the second a meeting room with an ellipsoidal shape.

As part of his practical consultancy work the author has encountered many more of these curved shapes, including circular auditoria, conical city council halls, and cylindrical meeting rooms. A number of these are mentioned in chapter 13 . The cases described in this chapter are those where more detailed research was possible and necessary.

\subsection{Some cases from the literature}

In the literature and many standard works on room acoustics (e.g., [Cremer78]) cases of sound focusing constructions are described.

Some well known examples are:

- $\quad$ The "ear of Dionysius" in Syracuse, Sicily (grotto),

- $\quad$ Grand Central terminal, New York (vaults),

- $\quad$ St. Paul's Cathedral (see par. 2.2) (cone),

- $\quad$ Statuary hall of U.S. Capitol building (ellipsoid).

In many of these cases there is only a (short) prosaic description of the geometry and the phenomenon available, but no detailed data or measurement data.

In this chapter we will describe some examples mentioned in the literature that contain more information. 


\subsubsection{Salt Lake City Tabernacle}

[Sabine22] indicates that the worst focusing effect (though he calls it "whispering gallery") is to be expected in an ellipsoid shape. He is able to name one (imperfect) example of such a room: the Mormon Tabernacle in Salt Lake City, Utah. A closer look reveals that this hall is a semi elliptical cylinder, at the ends covered with quarter-spheres or ellipsoids.

The hall actually is suffering from "whispering gallery" effects at many locations.

Recently measurements have been made of impulse responses, showing some echo effects [Rollins05], see figure 14.1. The source and receiver position are not the focal points.

The delay times roughly correspond to the reflection paths, as indicated in the calculation model shown in figure 14.1.
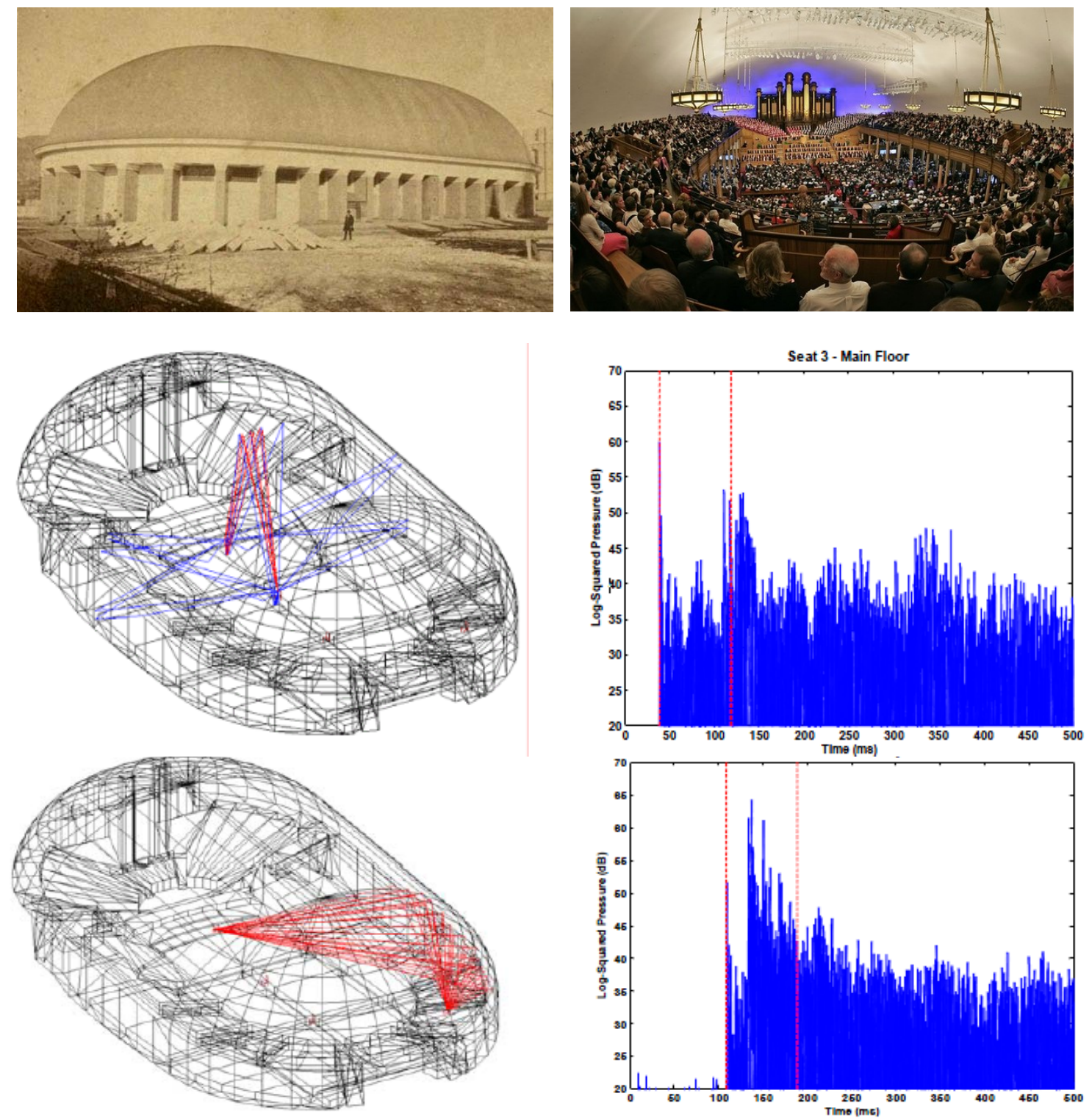

Figure 14.1. The Salt Lake City Tabernacle.Top: Photographs of the exterior in 1870 and the interior. Left: computer model indicating the relevant ray paths of the measurement results in the middle: Middle: receiver position at ground floor, Lower: receiver position at balcony [Rollins05] 


\subsubsection{Small theatre with cylindrical rear wall}

In [Mommertz95] and [Mommertz96] a situation of a small theater with a cylindrical rear wall is described.

The ground plan is shown in figure $14.2 \mathrm{a}$. The radius of the rear wall is $8,5 \mathrm{~m}$. For this situation measurement results are presented, which are shown in figure $14.2 \mathrm{~b}$ and 14.2c. The measurement position is $2 \mathrm{~m}$ from the source. The measurement shows a peak just before $50 \mathrm{~ms}(8,5+6,5=15 \mathrm{~m}$, this corresponds to ca. $45 \mathrm{~ms})$. The level of the reflection is $5 \mathrm{~dB}$ under the direct level at $2 \mathrm{~m}$ from the source.
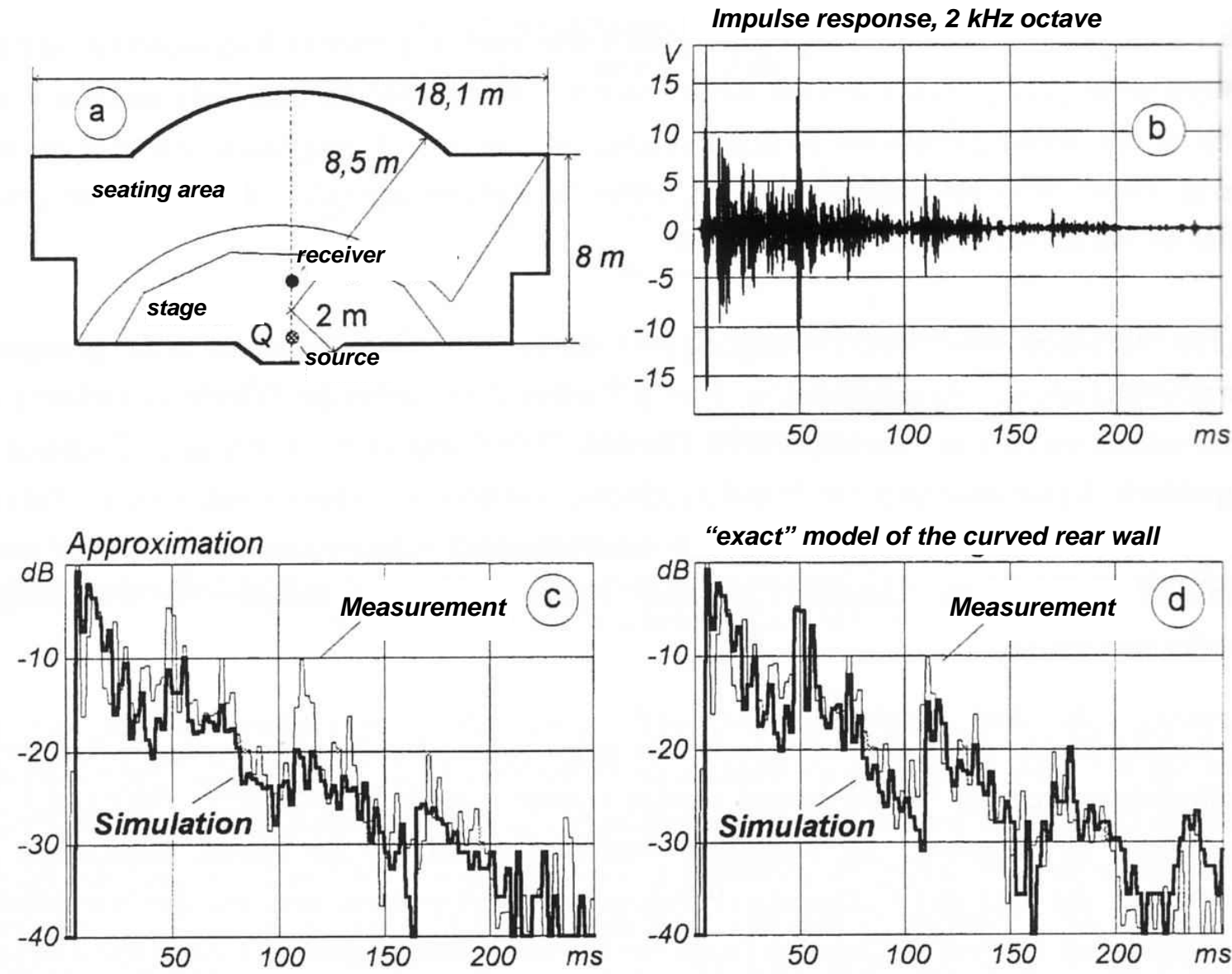

Figure 14.2. Small theatre with a cylindrical rear wall, described in [Mommertz96]. a: ground plan, b; impulse response, c: level-time graph of measurement result and simulation, d: leveltime graph of measurement result and improved simulation (ray tracing with curved surfaces)

The situation is also simulated using ray tracing. Figure $14.2 \mathrm{c}$ shows the result with a segmentation of the cylindrical rear wall into 5 segments. Figure $14.2 \mathrm{~d}$ shows the simulation result with the use of curved surfaces, which gives clearly better results.

A first approximation with the formulas given in this study would give the following results: For the measurement position at $2 \mathrm{~m}$, at $2 \mathrm{kHz}: x>>0,35 \lambda=x_{f}$ (8.32). This means that the measurement position is in the geometrical field, which can be approximated by using (5.17). A flat reflector would result in a reflection $L p=-23,5 \mathrm{~dB}$ (re $1 \mathrm{~m}$ ). $\Delta L_{c}$ according to (5.17) would be $+8,7 \mathrm{~dB}$, resulting in $-14,8 \mathrm{~dB}$ (re $1 \mathrm{~m}$ ), or $-14,8+6=-8,8 \mathrm{~dB}$ relative to the direct sound at $2 \mathrm{~m}$ from the source. This is a few decibels lower than the measurement result. 


\subsubsection{Haus des Lehrers, Berlin}

The reduction of an echo in a hall with a spherical ceiling, the "Haus des Lehrers" in Berlin, is described in [Tennhardt05]. The radius of the sphere is $22,27 \mathrm{~m}$, the base of the cupola is at $6,2 \mathrm{~m}$ above floor level and the maximum height is $13,2 \mathrm{~m}$. This means that the focusing points are well below floor level. Due to the converging sound field the sound pressure amplification can be estimated based on the geometrical field, according to (5.14). With source and receiver ca. $1 \mathrm{~m}$ above floor level, an amplification compared to the reflection of a flat surface is to be expected of about $9 \mathrm{~dB}$.

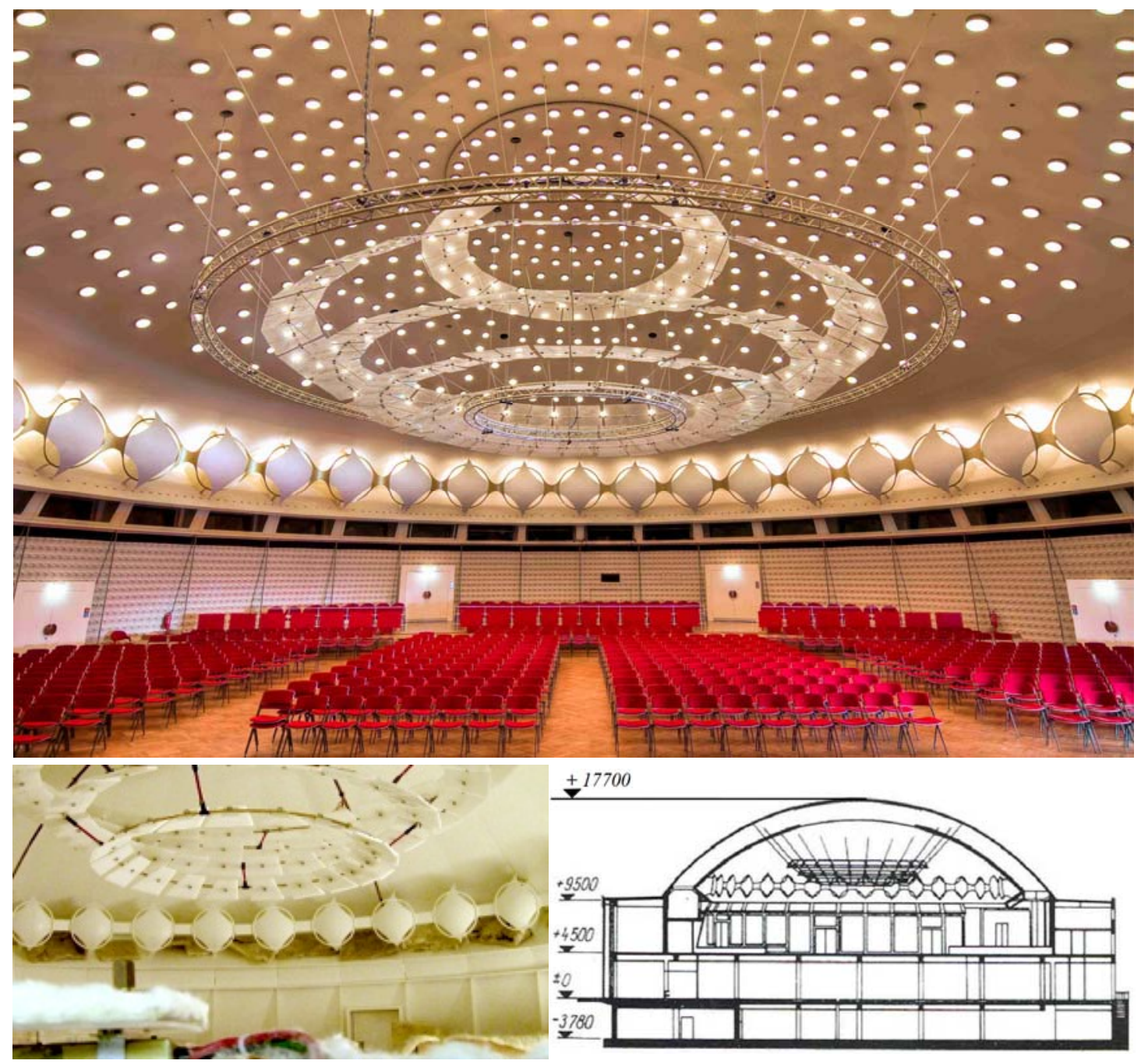

Figure 14.3. Kuppelsaal in Haus des Lehrers, Berlin. Upper: After renovation; ; Lower left: scale model 1:20; Lower right: section of the hall [Tennhardt05].

The echo occurred at about $60 \mathrm{~ms}$ and was disturbing. Due to the flat floor multiple reflections occurred, resulting in a clear flutter echo that strongly contributed to the disturbance. As part of the renovation in 2000 Tennhardt et al. performed research on a scale model scale 1:20. The echo was removed by a combination of diffusing elements on the cupola, free hanging reflecting panels (rings) and absorption in the central area of the dome. 


\subsubsection{Plenarsaal des Bundestags Bonn}

From the concept of a democratic arrangement of chairs, town halls and houses of parliament often have a (half) circular floor plan. If the metaphorical transparency of government is translated into glass separation walls, the circular walls have to be reflective.

After its opening, the hall of the German parliament in Bonn (which isn't used as parliament anymore since they moved to Berlin) showed a clear problem with the speech amplification system. This was especially a problem on the ground floor, which has a shape with cylindrical segments. These caused strong feedback on the microphones, which made it impossible to amplify the signal from the speaker sufficiently. The feedback was caused by the circular glass wall in combination with the high source position of the speakers. Further, multiple reflections were caused by
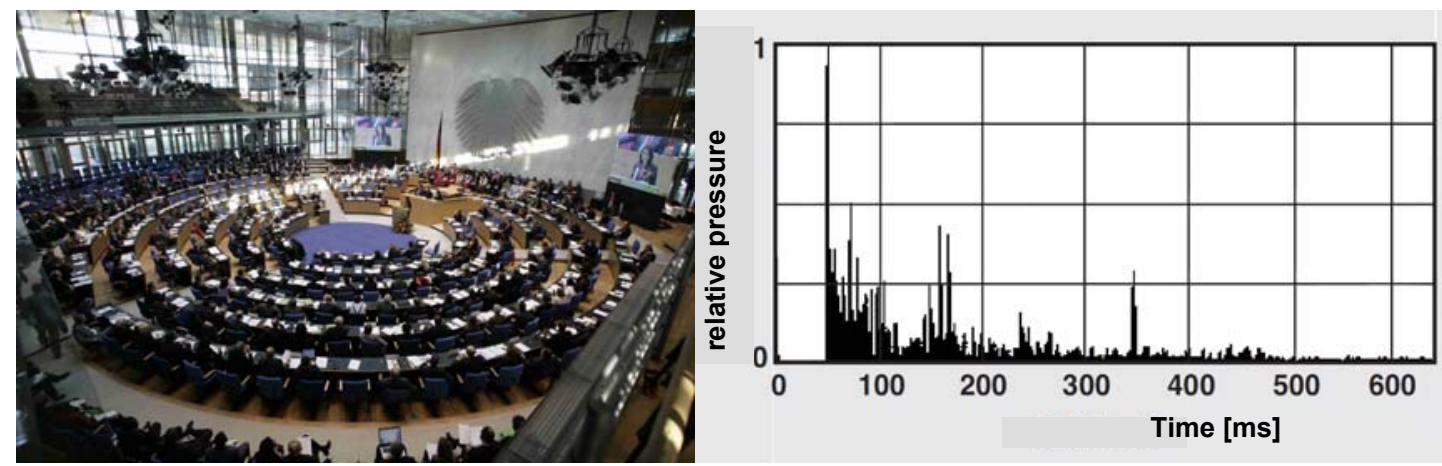

Figure 14.4. Plenarsaal des Bundestags Bonn (left) and the measured impulse response (right) showing clear echoes [Fasold94]

the flat rear wall (at $340 \mathrm{~ms}$ ), see figure 14.4.

After extensive calculations ([Stephenson94]) and scale model measurements [Fasold94], the problem was solved by a combination of

- redirecting reflections to the sound absorbing ceiling by glass panels (ca. $1,5 \times 1,5 \mathrm{~m})$, see figure 14.5 ,

- sound absorption on the wall with the eagle [Gertis94] ,

- sound absorption in front of the glass facades by acrylic glass with micro perforation [Fuchs97] ; the acrylic glass is also tilted to redirect remaining reflections to the absorbing ceiling, and

- improved directivity of the loudspeaker system, which directs the energy towards the seats as much as possible [Fasold94].

It is noted that, though the accuracy of the calculated sound field with ray tracing may be rather poor in the focusing point, the geometrical representation of the calculated rays in figure 14.5 can be a quite helpful communication tool.
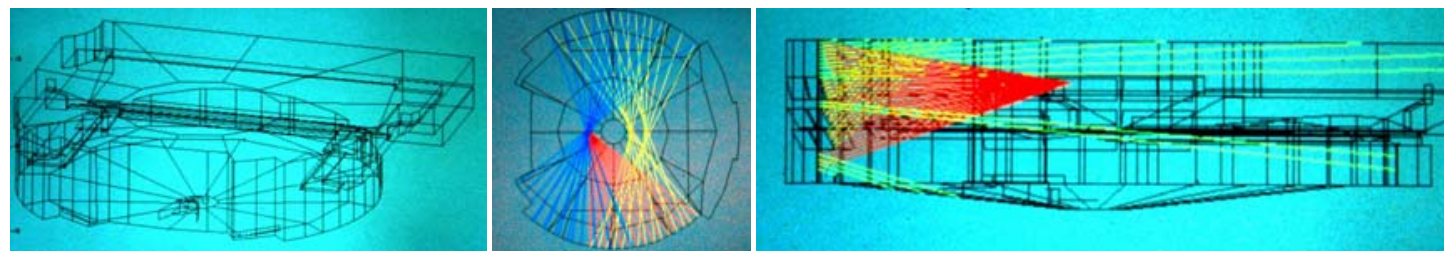

Figure 14.5. Computer model of the Plenarsaal der Bundestag in Bonn, Left: geometrical model, Middle: illustration of sound concentration, Right: redirecting energy to the floor and the sound absorptive ceiling [Stephenson08]. 


\subsubsection{Wigmore Hall London}

Wigmore Hall in London, formerly Bechstein Hall, is an interesting case since it has a ceiling that is shaped like an elliptical cylinder and it has a cylindrical apse on stage with a sphere segment on top. Although at first glance it seems to have all the ingredients for dreadful sound concentration effects, Wigmore Hall has excellent, very "strong" sounding acoustics. The hall is one of the best halls for chamber music and was used as an example for many other halls (see e.g., [Dillworth11] and the Aalmarkt Hall in Leiden).
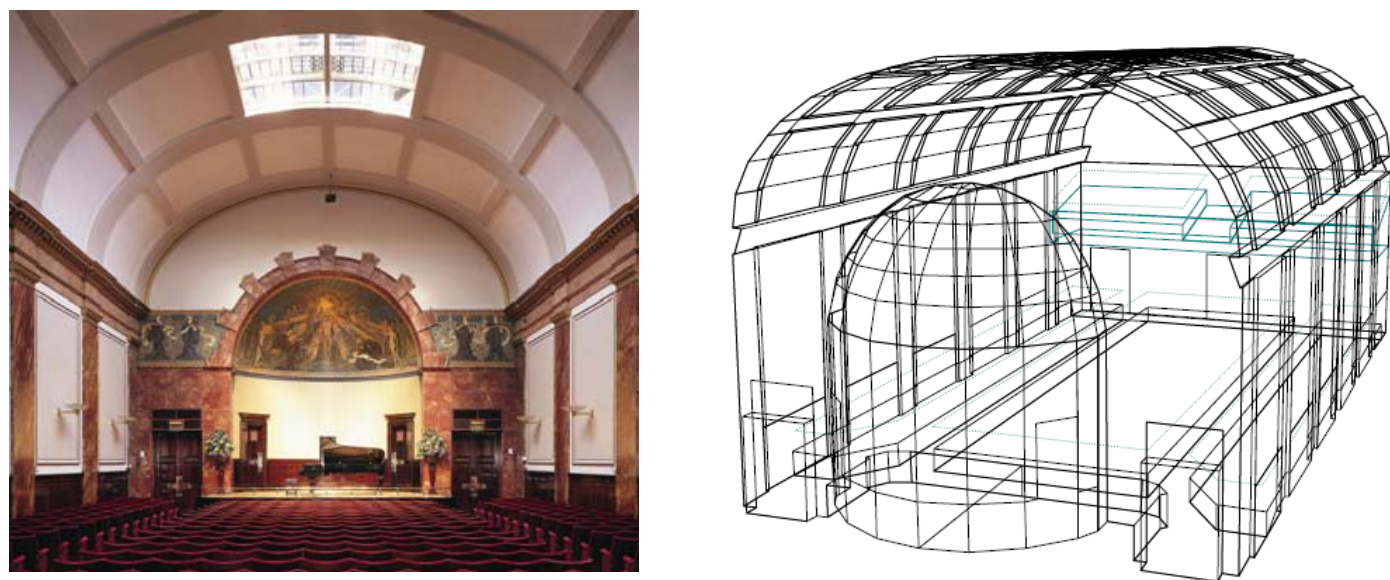

Figure 14.6. Interior of Wigmore Hall, view to the stage (left) and $3 D$ wire frame (right), continuous curved surfaces modeled as segments [Wulfrank06]

The hall has 542 seats and is $12,3 \mathrm{~m}$ wide, $22,9 \mathrm{~m}$ long and $10,7 \mathrm{~m}$ high, with a small balcony $3,5 \mathrm{~m}$ deep. Reflections from the concave ceiling will arrive between $20 \mathrm{~ms}$ (rear) to $37 \mathrm{~ms}$ (front) after the direct signal. At the balcony the delay is even less: around $10 \mathrm{~ms}$. Therefore the delay is shorter than the threshold of echo detectability (see figure 3.5).

An analysis of the hall's acoustic is given in [Wulfrank06]. The geometrically reflected sound field from the hall's ceiling and the apse is also described.

The effect from the cylindrical apse very much depends on the source and listener positions. For source positions between the centre point of the cylinder ("circular focus", see figure 14.7) and the focal point ("parabolic focus"), e.g., the cello, and the $2^{\text {nd }}$ violin, amplification will occur in the audience area.

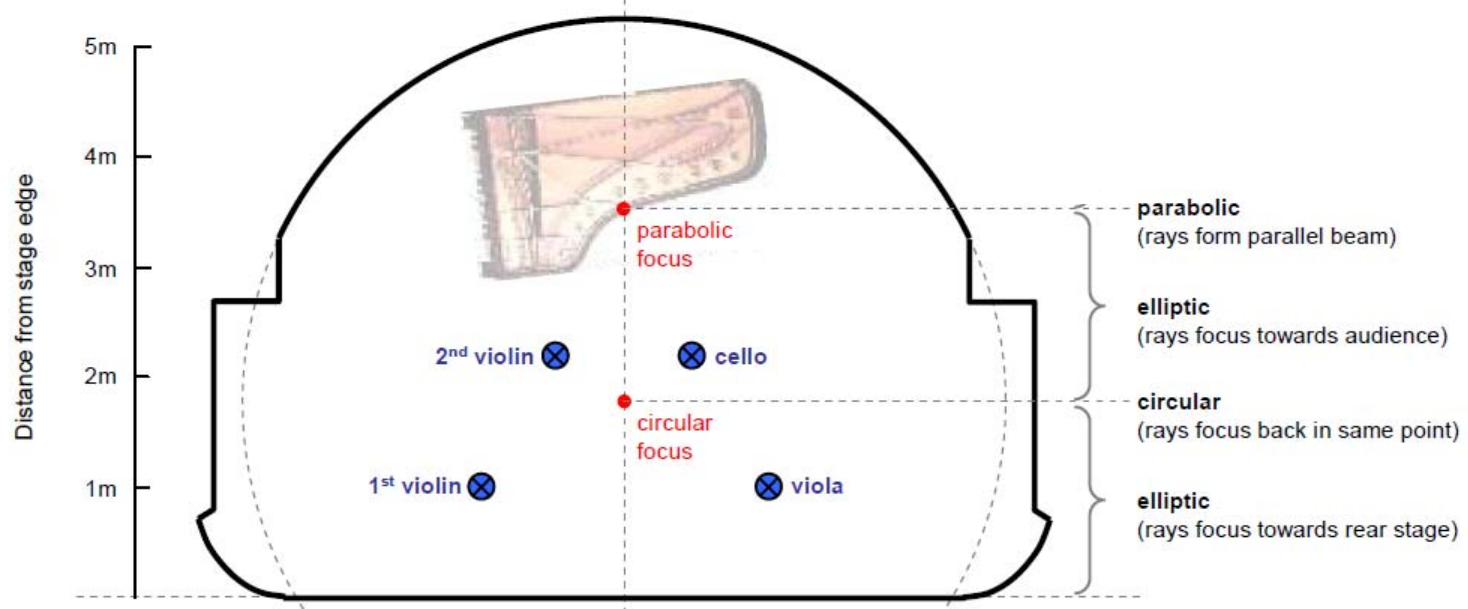

Figure 14.7. Stage at Wigmore Hall with typical source locations; right: zones indicating the reflection behavior from the cylindrical apse [Wulfrank06] 
The calculated amplification of the reflection $\Delta L_{c}$ for these source positions ranges from 3 to $5 \mathrm{~dB}$ for different audience positions [Wulfrank06].

The ceiling has an elliptical cross section with $a=6,15 \mathrm{~m}$ and $b=4 \mathrm{~m}$. It has a small opening in the ceiling, around $6 \mathrm{~m}^{2}$ that used to be closed by glass panels. The radius of curvature at the top is $9,4 \mathrm{~m}$, which reduces to $2,6 \mathrm{~m}$ at the base. This means that focusing is to be expected both in the stalls and in the balcony.

Measurement results of the impulse response in the stalls (in the centre at about $10 \mathrm{~m}$ from the source) are shown in figure 14.8. The opening in the ceiling was closed with panels. At a delay of $37 \mathrm{~ms}$ the sound level of the reflection is $3 \mathrm{~dB}$ below the level of the direct sound, which corresponds to $\Delta L_{c}$ of approximately $4 \mathrm{~dB}$. Without panels (so with the opening in the ceiling) the reflection is about $1 \mathrm{~dB}$ lower.

Measured at receiver position: $\Delta L=-3 \mathrm{~dB}$. With a reflection path of about $21,5 \mathrm{~m}$, the rel. SPL due to a reflection of a flat surface $\Delta L_{r}$ will be $-6,7 \mathrm{~dB}$. From (5.15): $\Delta L_{c}=3,7 \mathrm{~dB}$

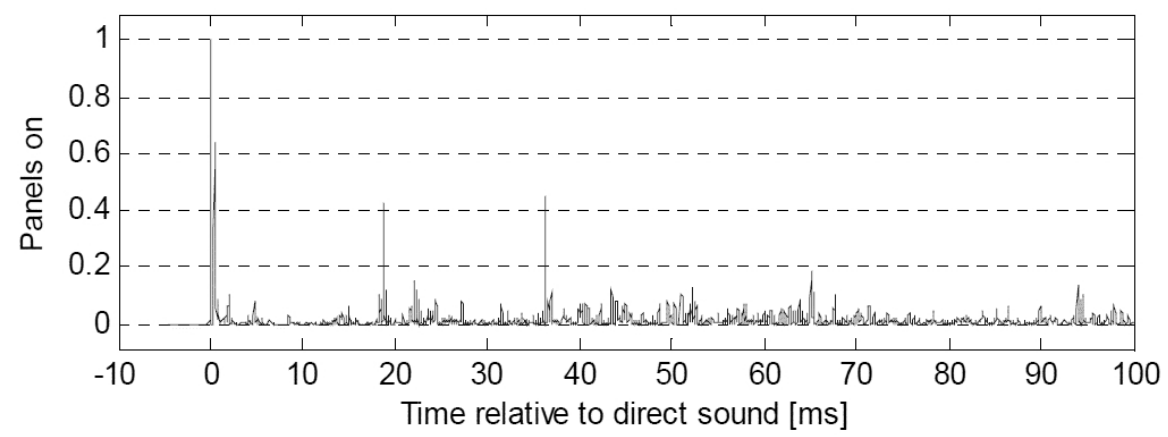

Figure 14.8. Measured impulse response (squared pressure) at seat H11 (about 10m from the source) with panels covering the daylight opening [Wulfrank06].

Since the geometry of this hall is documented rather well, it is worthwhile to use this case as an example to illustrate the methods described in chapter 12. So in addition to the work of [Wulfrank06], the sound distribution in the hall will be calculated here, both with the geometrical method as with the wave field method.

\section{Geometrical method}

Figure 14.9 shows a section of the hall with the geometrical paths (rays) indicated from a source at the centre of the stage. For each of these rays the reflection point in the hall is calculated as well as $\Delta L_{c}$ according to formula (5.17), using the radius of curvature from (5.22). The geometrical $\Delta L_{c}$ is shown in the graphs of figure 14.9 (dots). It shows strong amplification at the ground floor $\left(\Delta L_{c}>20 \mathrm{~dB}\right)$ in the centre of the hall, from the middle section of the ceiling. At balcony level the highest amplification $\left(\Delta L_{c}=12 \mathrm{~dB}\right.$ ) occurs between 1 and $2 \mathrm{~m}$ out from the centre and is caused by the ceiling section between 3,5 and $4,5 \mathrm{~m}$ out from the centre (indicated in the figure). The width of the geometrically calculated focusing areas is (unrealistically) small.

\section{Wave field method}

Since the geometry indicates focusing (also shown by the high $\Delta L_{c}$ ), a wave approximation is necessary. As a first step we may use formula (8.9c) to determine the focusing in a circular cylinder, applying the effectively reflecting area in the 
ceiling (for the stalls this is about $40^{\circ}$ ). This results in a $\Delta L_{c}$ of $7 \mathrm{~dB}$ at $500 \mathrm{~Hz}$ (see note below).

Note: application of the wave field method. If the source is assumed to be in the centre axis, (12.6) can be used to calulate the pressure on the centre axis:

$$
\Delta L_{1 m}=20 \lg \left(\frac{\theta_{m}}{\pi}\right)+10 \lg \left(\frac{\pi k}{R}\right)
$$

For focussing in the stalls, the input parameters are:

$$
\theta_{m}=40^{\circ} \text {, so } 20 \lg \left(\frac{\theta_{m}}{2 \pi}\right)=-19 \mathrm{~dB} ; R=9,5 \mathrm{~m} \text {; at } 500 \mathrm{~Hz}: k=9,2 \text {, so } 10 \lg \frac{\pi k}{R}=3 \mathrm{~dB}
$$

This results, from (12.6), in: $\Delta L_{1 m}=-16 \mathrm{~dB}$

Corresponding to (8.29) the SPL along the centre axis of the cylinder will gradually decrease:

$$
\Delta L_{f}=20 \lg \left(1+\left(\frac{z_{A}}{2 R}\right)^{2}\right)^{-3 / 4}
$$

In this case, with $z_{A}=10 \mathrm{~m}$, the reduction will be approximately $3 \mathrm{~dB}$.

The reflection from a flat ceiling ( $10 \mathrm{~m}$ from the source) will have a path length of $20 \mathrm{~m}$, so $\Delta L_{1 m}=-26 \mathrm{~dB}$, resulting in $\Delta L_{c}=7 \mathrm{~dB}$

However, since there can be some doubt about the determination of the effective size of the ceiling segment contributing to the focusing effect, numerical calculations have been performed using (6.19). Figure 14.10 shows the reflected sound field from the ceiling at ground floor and balcony level for a $500 \mathrm{~Hz}$ pure tone.

The results clearly show the focusing over a small area along the centre line in the stalls and over a wider range at balcony level.

Numerical calculation results for $250 \mathrm{~Hz}, 500 \mathrm{~Hz}$ and $1 \mathrm{kHz}$ pure tones are also indicated in the graphs of figure 14.9, showing that focusing will occur over a wider area than indicated by the geometrical method. Both in the stalls and in the balcony the focusing effect is a little higher than the first approximation based on (8.9c), indicating that the effective size of the ceiling segment contributing to the focusing is larger than the size determined by drawing rays.

With $\Delta L_{c}$ in the range of $10 \mathrm{~dB}$ the calculation results are also higher than the measurement result. One explanation could be the diffusion of the ceiling, but this could only partly explain the difference. Another explanation could be the exactness of the measurement position, since the SPL is very much place dependent.

For a good comparison and analysis more detailed measurements would be required. 


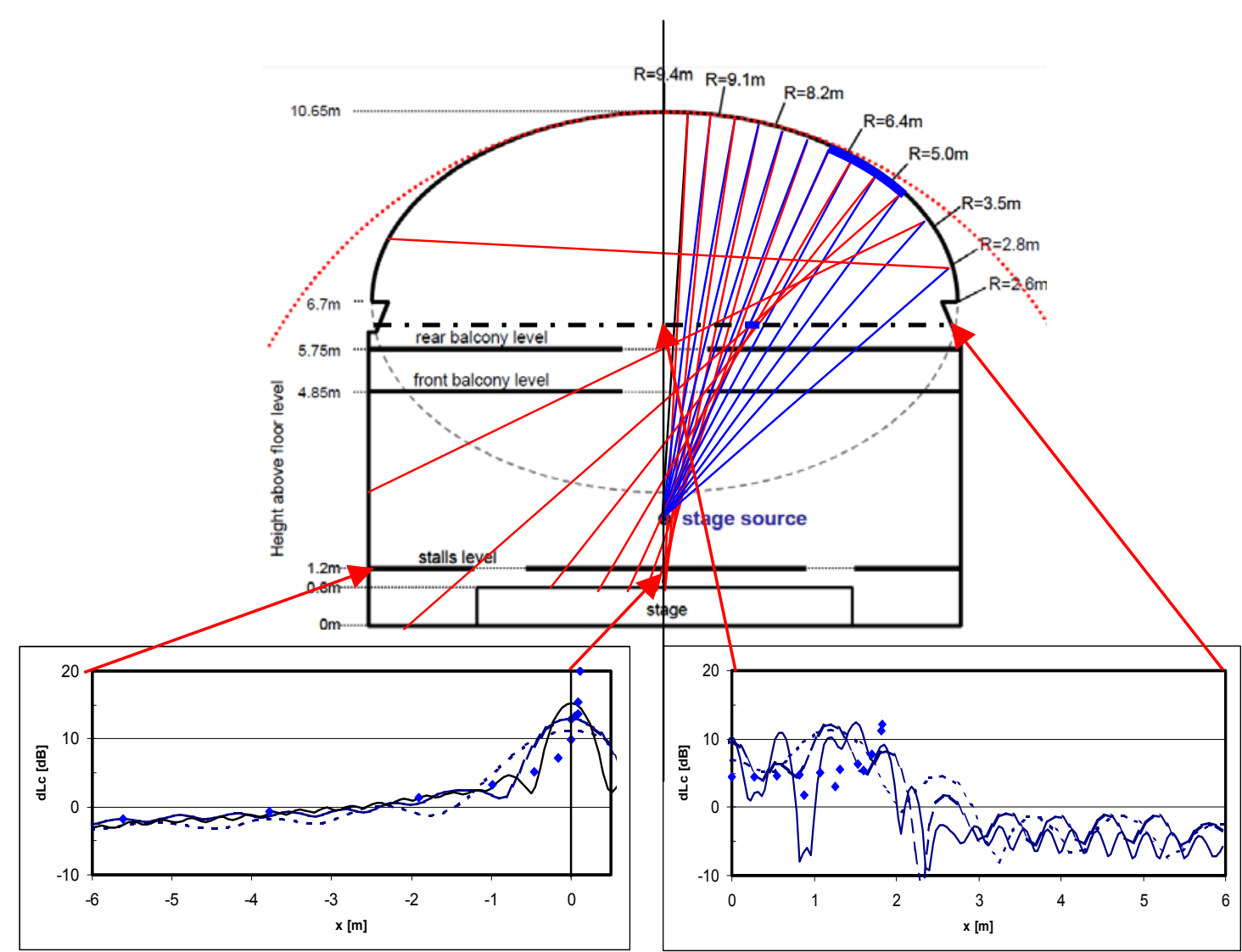

Figure 14.9. Cross section of Wigmore Hall with the ray paths incident on the ceiling indicated (one sided) as well as the geometrically reflected rays. The graphs below show the amplification of the sound level $\Delta L_{c}$ along a line. Left: stalls, $h=1.2 \mathrm{~m}, 8 \mathrm{~m}$ away from the source, Right: balcony $h=6.05 \mathrm{~m}, 18 \mathrm{~m}$ away from the source. Dots: geometrically calculated $\Delta L_{c}$ from (5.17) and (5.22); lines: numerically calculated reflection from ceiling $\left(\Delta L_{c}\right)$ using (6.19); solid line: $1000 \mathrm{~Hz}$, dashed line: $500 \mathrm{~Hz}$, dotted line: $250 \mathrm{~Hz}$.

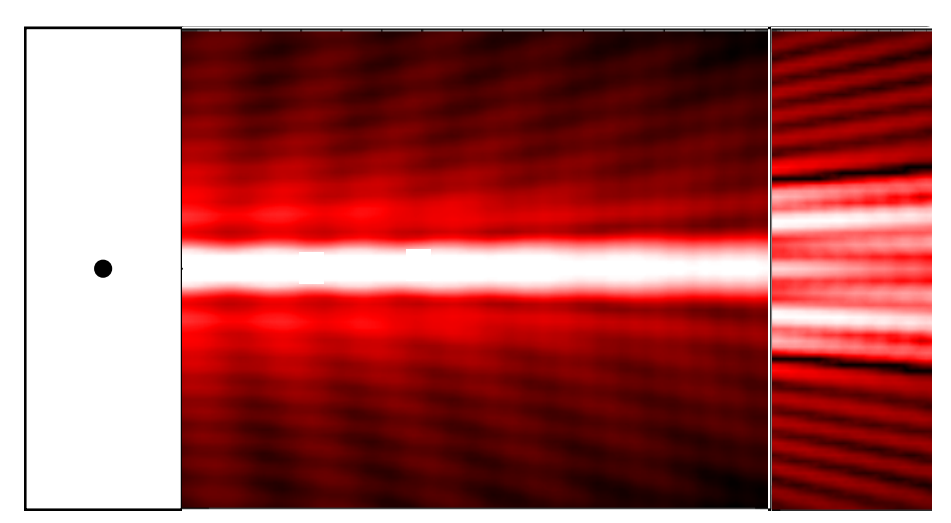

Figure 14.10. SPL of reflected field in Wigmore Hall, $500 \mathrm{~Hz}$. Left: source position on stage, centered; middle: stalls, right: balcony. Calculated using (6.19). white: $\geq-15 \mathrm{~dB}$, Black $\leq-35$ $d B$ (relative to the SPL at $1 \mathrm{~m}$ from the source) 


\subsection{Scale model research}

Scale model research allows the evaluation of the acoustic of a new hall or the evaluation of the efficiency of alterations for existing halls, at lower costs and effort as in real scale.

In this paragraph a short introduction into scale model research will be given, because scale model research is applied for improving the acoustics of the Tonhalle Düsseldorf and the Royal Albert Hall London, see paragraphs 14.3 and 14.4.

The use of scale models for room acoustical problems is not limited to acoustical scale models.

Since early in the $19^{\text {th }}$ century optical models have been used to visualize the first reflection from surfaces (see e.g., [Cremer78]). The advantage of such a method is the clear visualization; the disadvantage is that, since the wavelength of light is very small compared to the surfaces, it is a high frequency model. Diffraction is not included.

But even now, it can be helpful to use mirrors and light sources as a first step to locate focusing points (see figure 14.11). Since focusing is a local phenomenon, one must actively search for the focusing position(s), before performing the acoustic test.

Another (old) scale model technique (see e.g., [Cremer78]) is the use of two dimensional models in a water bath. The wave propagation is studied with surface waves. Due to the low speed it is nice for demonstrations. The advantage of the method is the fact that bending and diffraction is included. One of the disadvantages is that it is limited to two dimensions, for example, one section of a room.

A comparable scale model technique involved a very short duration light bundle on photographic paper. The actual sound wave in an 2D model caused refraction of the light wave and thus the sound wave was made visible (see e.g., figure 14.12 and [Cremer78], [Sabine22]).

Due to the development of electro-acoustical transducers, especially since the introduction of impulse response measuring techniques, the use of acoustical scale models is a practical and valuable tool for evaluating and improving the room acoustics of halls. It can be used when geometrical computer models are not sufficiently accurate, such as in the case of curved surfaces or for concert halls where the structure of early reflections is important.

The concept of the acoustical scale model is that the wavelength $\lambda$ in the model is changed, corresponding to the scale factor of the model. In the case air is being used in the model, the sound velocity is unchanged. Since the frequency is inversely proportional to the wavelength according to: $f=c / \lambda$; the frequency scales with the same scale factor. Using scale factor 1:10 requires the frequency to be scaled up with a factor 10 . This sets the requirements for the sound sources, microphones and materials used in the scale model. It may also limit the frequency range that can be studied.

For example, the evaluation of the impulse response will to a large extent be based on the $1 \mathrm{kHz}$ band, extending to approx. $1400 \mathrm{~Hz}$. This means that, using scale 1:10, the sources and microphones have to be sufficiently adequate up to $14 \mathrm{kHz}$. This can be done without much difficulty.

Impulse responses measured in a scale model, especially when made with a dummy head, can also be used for auralisation. A sound (music, speech) will be made audible by convolution of the source signal (free from reflections, recorded in an anechoic room) with the measured impulse response, in this case measured in the scale model. In such a case the high frequencies play an important role and $14 \mathrm{kHz}$ as an upper limit would certainly not be adequate. 


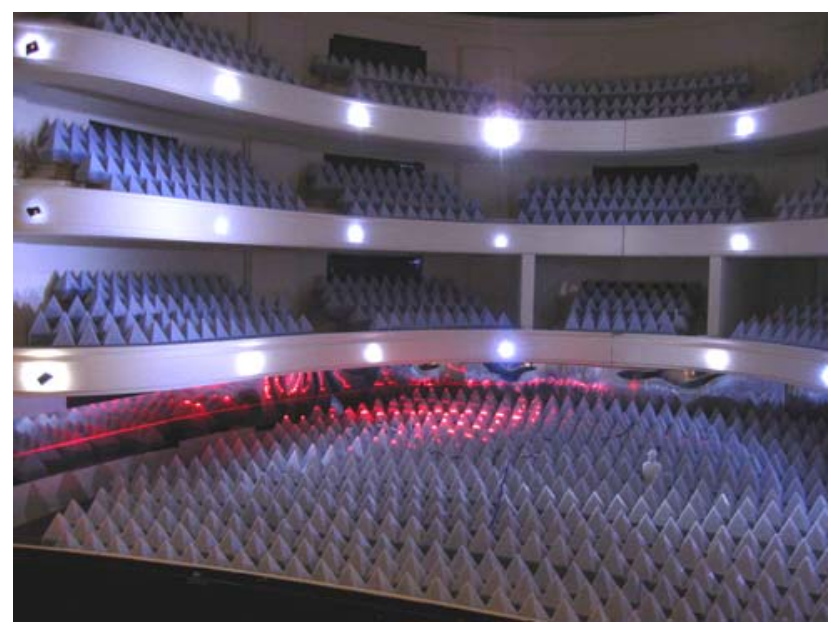

Figure 14.11. Acoustic scale model 1:10 of the Berlin Staatsoper Unter den Linden. The circular wall at ground floor is covered with a flexible mirror. With the help of several (red) laser sources on stage, the geometrical reflection is visualized.
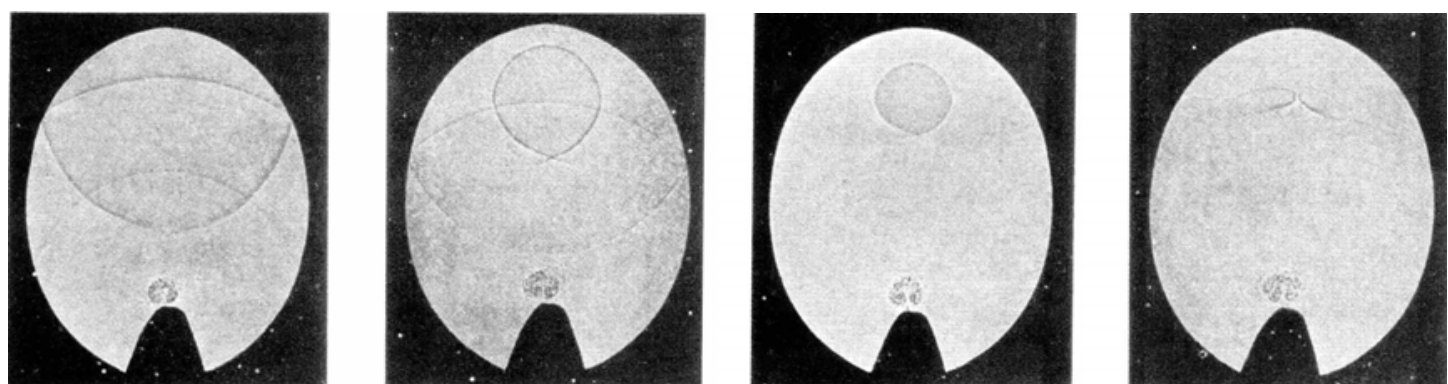

Figure 14.12. Photographs illustrating how the sound emitted in one focal point concentrates in the other focal point of an ellipse [Sabine22]

The sources and microphones used in the studies presented in par. 14.3 and 14.4 have sufficient signal to noise ratio and are sufficiently omni-directional for frequencies up to approx. 40 or $50 \mathrm{kHz}$, but do not have a flat frequency response (see also Chapter 11). In specific conditions one might consider using sources with the radiation characteristic of a speaker. For the research performed in the cases

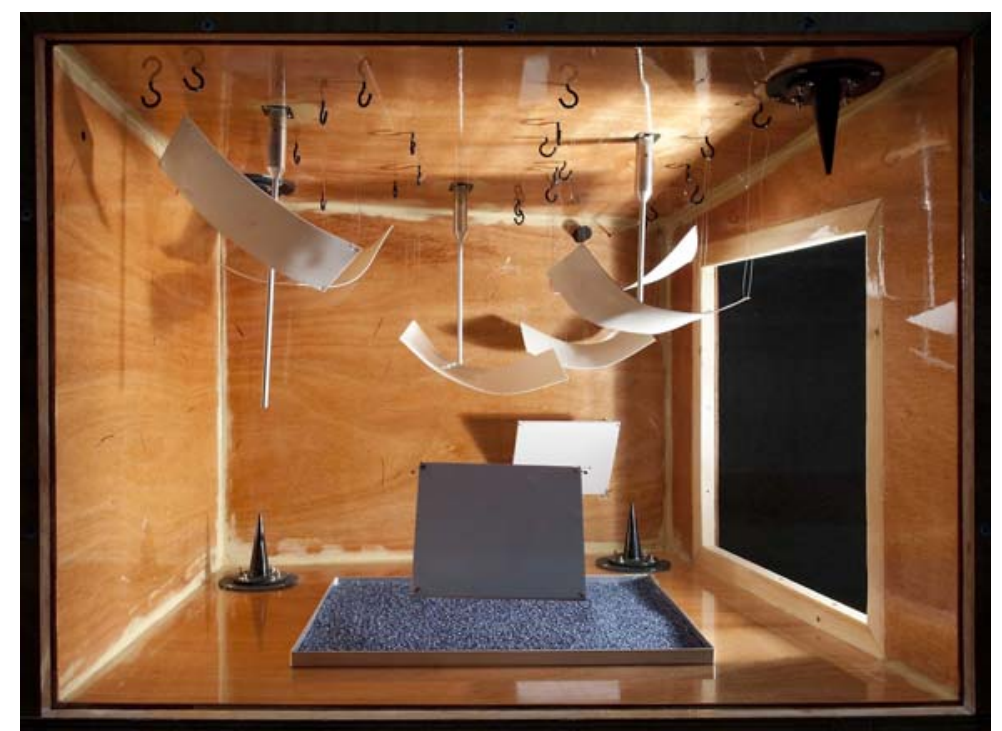

Figure 14.13. Scale model of the reverberation chamber 1:10. Sources: black inverted conical horns at the floor, rear left and rear right (and at the ceiling, front right); the drivers of the sources are built into the model. Microphones: three, hanging from the ceiling [Lautenbach10] 
presented here omni-directional sources were needed that corresponded to the sources required for real scale measurements in [ISO 3382-1].

Since the point sources have a relatively low output power and the small microphones have a high noise level, the dynamic range of scale model measurements is much more limited than real scale measurements.

The signal to noise ratio is sufficiently high for evaluating the impulse responses in the octave band of 2 and $4 \mathrm{kHz}$, but is still rather low for high quality auralisation.

The sound absorption of the materials applied in these scale models have to be "scaled" according to the scale factor as well. The material in the model should have approximately the same absorption characteristics as the real material at real scale. The exactness of these properties, however, depends on the goal of the research. When compared to computer modeling, the advantage of a physical scale model is its more correct representation of single or multiple reflections. The scale model is generally not able to obtaining more accurate reverberation times than computer models. For studying early reflections, the scale models' results are only moderately dependent on exact absorption characteristics.

The absorption of scale model materials is determined according to [ISO 354] in a scale model reverberation chamber. Figure 14.13 shows a picture of a 1:10 scale reverberation chamber, also showing the sources and microphones used.

Typical materials used in scale model research are mdf (medium-density fibreboard), painted to reduce the surface porosity, and $2 \mathrm{~mm}$ polystyreen for smaller or more complicated reflecting parts and pyramid foam for audience absorption.

The main difficulty in acoustic scale model testing is that the absorption of air is not scaled according to the scale factor. The mechanisms for sound absorption in air are classical absorption and molecular absorption. Classical absorption is energy dissipation by viscous and thermal losses due to friction between molecules. This absorption is proportional to the square of the frequency. Molecular absorption is caused by relaxation processes of oxygen and nitrogen and is strongly influenced by water vapour molecules. The combination of the two mechanisms results in a complicated dependency on gas properties, pressure, temperature, humidity and frequency. A model of this relationship can be found in [ISO 9613] (see also [Boone94]). Since the absorption in a scale model is much higher (more than the scale factor) than in real scale, especially at high frequencies, a way must be found to reduce this absorption.

One possibility is to reduce the oxygen content by filling the model with a gas with low absorption, for example nitrogen. This imposes practical problems due to the limited air tightness of models and the amount of time and nitrogen needed to fill large scale models, as well as health risks due to the effects of nitrogen and lack of oxygen. The latter is a very serious risk, since people must enter the model to make alterations or move measuring equipment.

A second possibility would be to use air with very low relative humidity, but this is still rather complicated from a practical point of view [Xu11].

Another alternative is computational correction of the impulse response, where negative time dependent correction is applied to the decay, lifting the slope of the decay. The disadvantage of this method is that the noise tail is also amplified (see e.g.,[Lorenz01], this can e.g., be observed in figure 14.34, left). The computational correction method is sufficiently reliable for frequencies up to $25 \mathrm{kHz}$.

Based on practical considerations, the computational correction method is used in the studies presented in the next paragraphs.

Although scale model research can be invaluable for testing different solutions and variations, it should be realized that the results can still differ from reality. Apart from the air absorption, the modeling rules are rigorous and the accuracy is high. The 
limitations are in the properties of the instrumentation (mentioned above) and in the detail of the model. In general, details smaller than $10-20 \mathrm{~cm}$ (on real scale) are not modeled anymore.

As with other kinds of modeling, absolute values may be of limited value. Comparison between variants, however, can be quite accurate and meaningful. The only difficulty in this comparison, however, is posed by a change of climatic conditions. Differences in temperature cause differences in the sound propagation velocity, this causes small differences in the interference pattern and therefore in the fine structure of the impulse response. Although these differences are much smaller than when placing the microphone one seat away, in the process of comparing measurement results due to small changes to the room, the variations in impulse response due to the temperature influences may hide the actual changes in the room. For that reason it is helpful to have a constant temperature in the scale model, as well as well fixed source and microphone positions.

Although scale model research has its limitations, it is the best practicable and accurate tool to predict sound concentration due to curved surfaces, especially in complex situations. 


\subsection{Tonhalle Düsseldorf}

The Tonhalle Düsseldorf is a hall for symphonic music and the main performance hall of the Düsseldorfer Symphoniker. Originally it was part of an exhibition building dating from 1928. The hall used to be a planetarium. After the Second World War, when the old shoebox type concert hall with the same name was destroyed, the space was used as a multipurpose hall, called the Rheinhalle. In 1978 the present concert hall was realized. Before the renovation in 2005, the Tonhalle Düsseldorf had a shape close to a hemisphere. The volume was about $15.000 \mathrm{~m}^{3}$ (after renovation ca. $16.000 \mathrm{~m}^{3}$ ) and it had 1933 seats (after renovation 1854 seats).

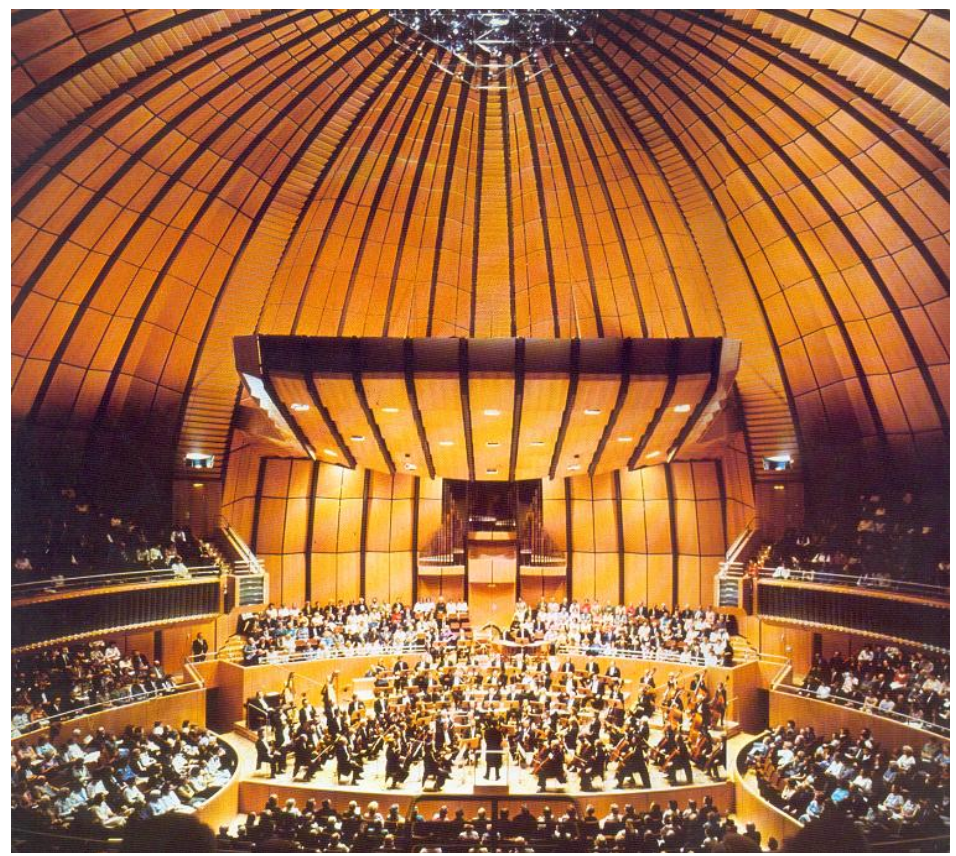

Figure 14.14. Interior of the Tonhalle Düsseldorf before the renovation of 2005

The hall had a bad reputation because of its strong echo ("Klopfgeist" or "knocking ghost"), which was especially disturbing with impulsive instruments like percussion and piano. Other problems related to the dome reflection were the unbalance of orchestral instruments in the parterre and the short reverberation due to the single reflection into the absorbing audience area.

\subsubsection{Geometry}

A section and ground plan is shown in figure 14.15. In the lower part, below the balcony, the walls were vertical. Above the balcony the dome starts. The radius of the dome was about $19,5 \mathrm{~m}$ and its centre was $1,2 \mathrm{~m}$ above stage level. The dome reflection occurred after $115 \mathrm{~ms}$. At eight positions, corresponding to the entrance doors, there was an irregularity in the shape of the dome, which gradually disappeared at a greater height. The inner dome was made of flat wooden panels with slits between them, which realized some low frequency absorption. The wooden panels were positioned at a slight angle in the radial direction, which also disappeared at a higher elevation; this angle caused some diffusion.

A large orchestra reflector prevented part of the sound energy from reaching the dome. 

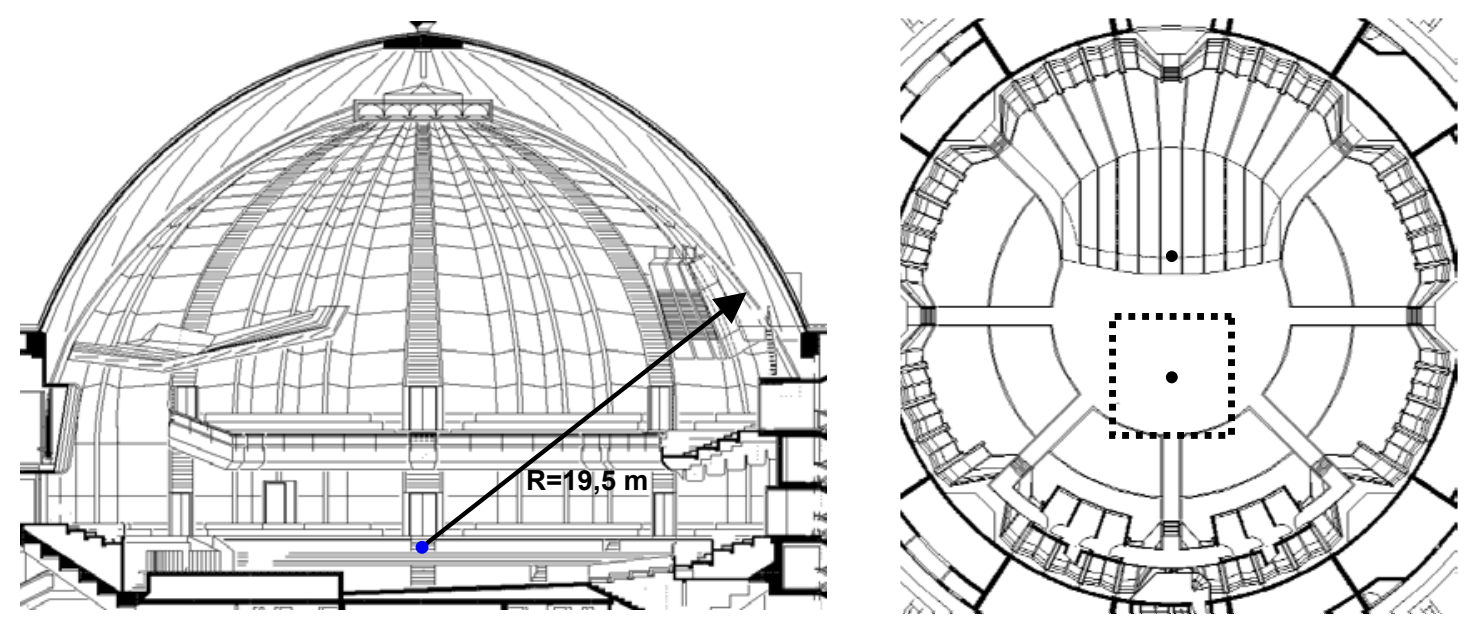

Figure 14.15. Section (left) and ground plan (right) of the Tonhalle Düsseldorf, before the renovation of 2005. Centre and radius of the dome are indicated in the section. The ground plan also shows source position, focus position and dotted range used in the calculation of figure 14.17

\subsubsection{Approximation based on the basic geometry}

Source in the centre

The total visible reflecting concave surface is calculated to be approx. $50 \%$ of a hemisphere (not considering the stage reflector). With a sound source in the centre of a geometrically perfect dome, the reflected sound pressure in the centre can be calculated from (12.3). For $500 \mathrm{~Hz}$ this results in a maximum pressure of $\Delta L_{1 m}=13 \mathrm{~dB}$ (SPL relative to the SPL at $1 \mathrm{~m}$ from the source).

\section{Source outside the centre}

For a source position $4 \mathrm{~m}$ from the centre of the dome, the reduction of the pressure in the point symmetrical focusing point can be derived from (12.5). However, in this case the distance $x$ of the source position to the centre at $500 \mathrm{~Hz}$ is equal to $1,1 \sqrt{R \lambda}$, which is more than allowed for application of (12.5): $x<0,85 \sqrt{\lambda R}$. Application of (12.5) would result in a reduction $\Delta L_{f}$ of about $7 \mathrm{~dB}$. With the use of (7.31) a reduction $\Delta L_{f}$ of $6 \mathrm{~dB}$ is obtained.

If the source is $4 \mathrm{~m}$ from the centre and the receiver point at $8 \mathrm{~m}$ from the source, the direct sound will be $18 \mathrm{~dB}$ lower than the SPL at $1 \mathrm{~m}$ from the source. The $\Delta L$, the SPL of the reflection from a geometrically perfect dome, relative to the SPL of the direct sound, will be $13-6+18=25 \mathrm{~dB}$ at $500 \mathrm{~Hz}$

It is obvious, however, that the dome is not a geometrically perfect dome. About $2 / 3$ of the panels do follow the dome shape roughly but are also slightly tilted in the radial direction. So the accuracy of this approach based on the basic geometry might be rather limited. The next step is a more accurate calculation based on the actual geometry of the dome. 


\subsubsection{Numerical approximation (before renovation)}

A computer model of the Tonhalle before renovation was made with approx. 1500 flat surfaces. Each panel is meshed according to par. 6.5. Only the vertical panels forming the circular basis and the dome panels are modeled. The slits between the panels are not modeled. Balcony and orchestra reflector are not considered here. An impression of the model is shown in figure 14.16.

The first calculations are performed with the source located in the centre of the dome (1.2 $\mathrm{m}$ above stage). The calculated pressure as a function of frequency can also be found in figure 14.16. It shows that at lower frequencies the results are close to the result obtained with (12.3) for a perfect geometrical dome. At higher frequencies the numerical result is lower than for the perfect dome (12.3). The main reason lies in the irregularities of the dome panels, which are more effective at diffusing high as opposed to low frequencies.

In a second step the source is located at stage, at $4 \mathrm{~m}$ from the centre, as shown in figure 14.15. The pressure is calculated in and around the focusing point, an area of $8 \times 8 \mathrm{~m}$, also shown in figure 14.15. The calculated reflected sound pressure level is given in figure 14.17. It shows that the focusing area is frequency dependent, as can be expected. Especially at higher frequencies the focusing occurs in a limited area.

The model is not exactly symmetrical to the longitudinal axis, so the calculation shows slightly imperfect left-right symmetry.

Note:

The required calculation time $T_{c}$ [s] of numerical calculations over an area, as shown in figure 14.17 can be estimated with

$$
T_{c}=T_{i} S_{r} S_{c}\left(\frac{k n_{\lambda}}{2 \pi}\right)^{4}
$$

with: $\quad T_{i}$ calculation time [s] per surface element, this is dependent on the computer.

For the calculations in this study it was about $3.2 \cdot 10^{-5} \mathrm{~s}$

$S_{r}$ total area of the reflecting surface $\left[\mathrm{m}^{2}\right]$

$S_{c}$ area of the receiver points $\left[\mathrm{m}^{2}\right]$

$n_{\lambda}$ number of wavelets per wavelength

For the calculation at $500 \mathrm{~Hz}$ in figure 14.17 the calculation time was $8,5 \mathrm{~h}$. The meshing of the geometry and calculating the incident sound relates to $k^{2}$ and took about $5 \mathrm{~s}$.

More detail can be found in the graph in figure 14.18, showing the pressure along the centre line from the centre point ( $4 \mathrm{~m}$ from the source) to the position $12 \mathrm{~m}$ from the source, corresponding to the centre line in the results shown in figure 14.17. The figure shows the SPL of the reflection relative to direct SPL. At the focusing point the reflection is about $17 \mathrm{~dB}$ stronger than the direct signal, which is about $8 \mathrm{~dB}$ weaker than the prediction based on (12.3). Between 6 and $8 \mathrm{~m}$ from the centre the reflection is still about $10 \mathrm{~dB}$ stronger than the direct signal for 250 and $500 \mathrm{~Hz}$. 

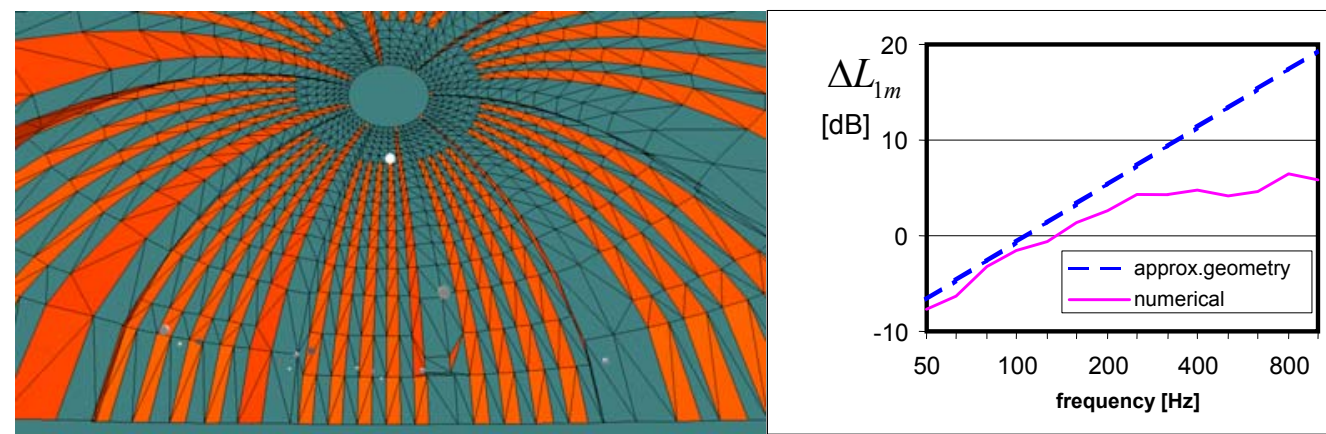

Figure 14.16. Interior view in the numerical calculation model of the Tonhalle Düsseldorf (left) and right: calculated $\Delta L_{1 m}$, the reflected SPL according to (6.19) in the centre (source also in the centre), relative to the SPL at $1 \mathrm{~m}$ from the source. Also shown: the approximation based on the basic geometry according to (12.3).

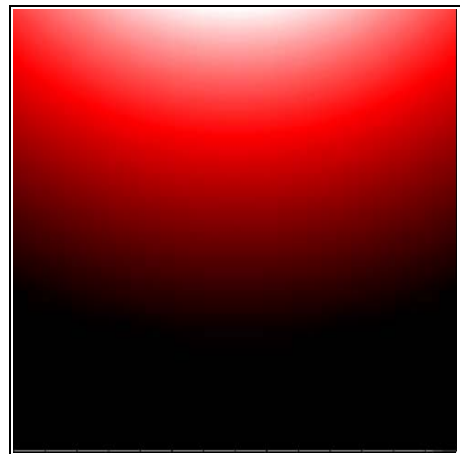

direct sound

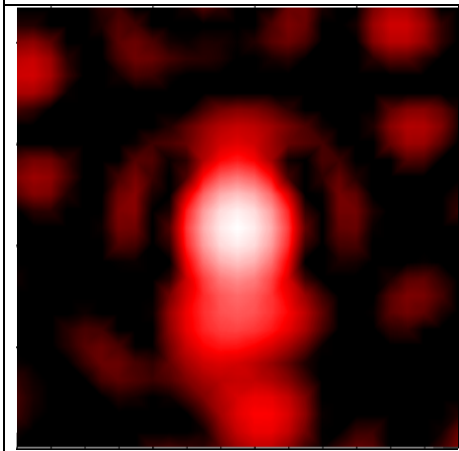

$125 \mathrm{~Hz}$

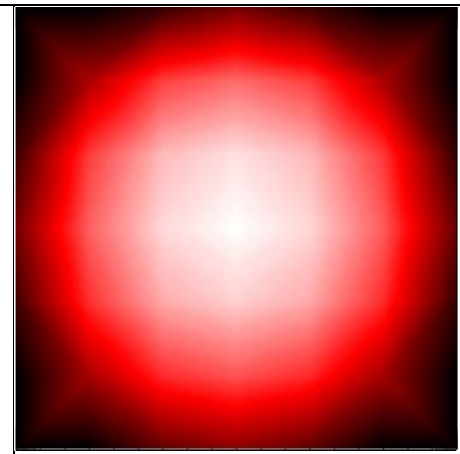

$31 \mathrm{~Hz}$

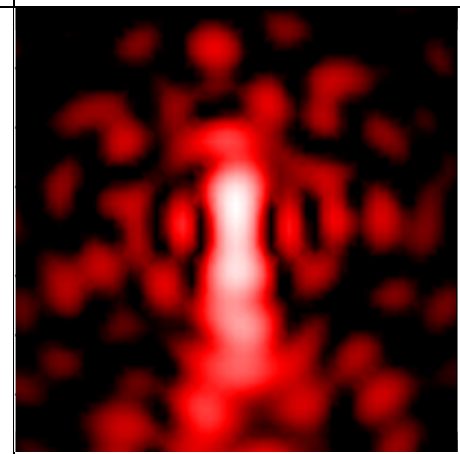

$250 \mathrm{~Hz}$

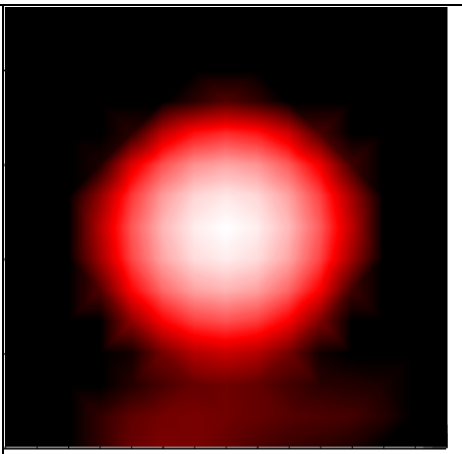

$63 \mathrm{~Hz}$

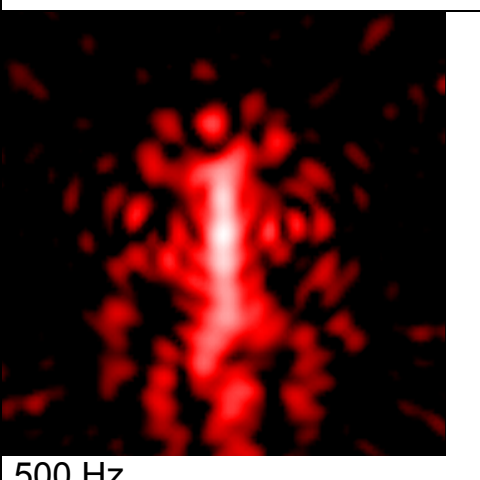

Figure 14.17. Calculated direct SPL (upper left) and reflected SPL (re $1 \mathrm{~m}$ ) at the parterre in the Tonhalle Düsseldorf. Numerical calculation using (6.19). white: $\geq 0 d B$, black $\leq-20 d B(r e$ $1 \mathrm{~m})$. Calculation range: see dotted area in figure 14.15.

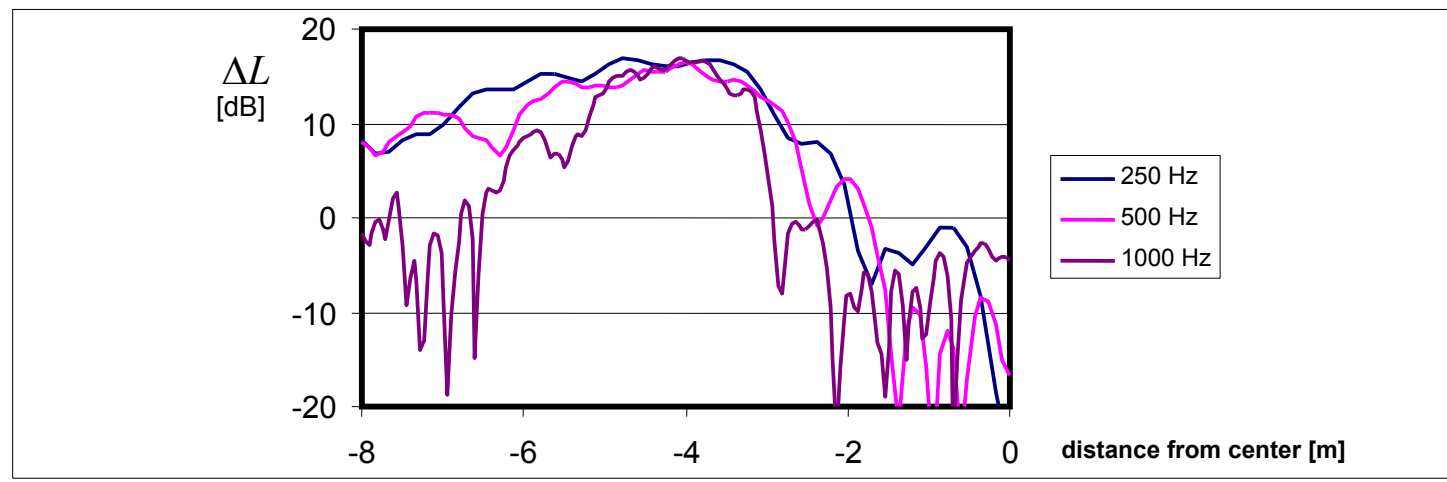

Figure 14.18. Calculated $\Delta L$, the reflected $S P L$ relative to the direct sound, at the parterre, along the longitudinal axis, in the Tonhalle Düsseldorf. Numerical calculation using (6.19). Pure tone frequencies of $250 \mathrm{~Hz}, 500 \mathrm{~Hz}$ and $1 \mathrm{kHz}$. 


\subsubsection{Measurements in the hall before renovation}

Before the renovation of the Tonhalle, impulse response measurements are performed at several positions in the hall. The measurements are performed with a MLS measuring system. The results are presented as log-square values (octave band filtered energy-time curve ETC).

Figure 14.19 shows the measurement result for source-receiver positions almost symmetrically to the centre. The amplification found (level difference between the reflected sound from the dome and the direct sound) was about $16 \mathrm{~dB}$ at $250 \mathrm{~Hz}, 14$ $\mathrm{dB}$ at $500 \mathrm{~Hz}$ and $<0 \mathrm{~dB}$ at $1 \mathrm{kHz}$. At $1 \mathrm{kHz}$ the reflection from the dome is clearly weaker, but this is position-dependent; at other positions the $1 \mathrm{kHz}$ reflection can be stronger. The amplification in the different octave bands depends on the exact position.

The dome reflection arrives at about $110 \mathrm{~ms}$. The delay is around $80 \mathrm{~ms}$.

At $500 \mathrm{~Hz}$ and $1 \mathrm{kHz}$ an additional peak at about $80 \mathrm{~ms}$ can be observed. This peak is due to the reflection from the low cylindrical parapet in the audience area. This peak is less pronounced when an audience is shielding these reflection surfaces.

The next step is to evaluate the peak and determine how much it exceeds the criteria described in chapter 3 , since this will be the requirement for the reduction of the echo.

\section{Echo detectability according to Makrinenko}

The threshold for echo detectability for speech according to formula (3.1) is $-18 \mathrm{~dB}$ at $80 \mathrm{~ms}$. The measurement results in the Tonhalle significantly exceed this value, by about $32 \mathrm{~dB}$. However, this value does not incorporate the influence of other reflections.

\section{Reflections in the reverberant field}

The level of other reflections from non-concave surfaces may be estimated using (3.6).

The expected SPL of "normal" (full) reflections will be ca. $\Delta L_{r}=-11 \mathrm{~dB}$ relative to the direct level at $10,5 \mathrm{~m}$ from the source. This means that the actual reflection at $500 \mathrm{~Hz}$ is about $11+14=25 \mathrm{~dB}$ above the level of "normal" reflections.

\section{Method of Yamamoto}

Figure 14.20 gives an analysis of the measured impulse response for the $500 \mathrm{~Hz}$ octave band according to the method of Yamamoto, which is described in par. 3.4. The first peak exceeds the threshold by $5 \mathrm{~dB}$, the second by $9 \mathrm{~dB}$. If the first peak is $5 \mathrm{~dB}$ lower with the audience present, the dome reflection will exceed the threshold by approx. $14 \mathrm{~dB}$.

\section{Method by Dietsch and Kraak}

The evaluation of the echo according to [Dietsch86] (see also par 3.5) is given in figure 14.19. At 250 and $500 \mathrm{~Hz}$ the recommended values of 0.9 for speech are clearly exceeded. The value of the echo criterion for music (1.5) is barely exceeded, which does not seem to match the obvious annoyance caused by the echo in this hall. The presence of the early peak does have a masking influence in this evaluation method.

From the different evaluation methods it was concluded that the required reduction was 15 to $20 \mathrm{~dB}$. 


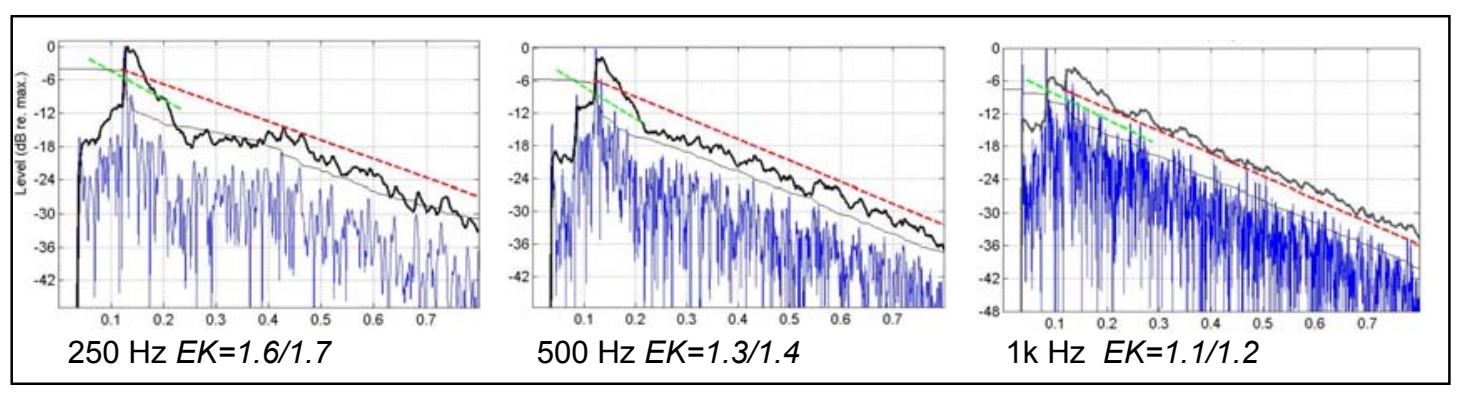

Figure 14.19. Measured impulse response, presented as Energy Time Curve (ETC) at the echo position in the Tonhalle Düsseldorf without audience, before renovation (receiver at ca. $10,5 \mathrm{~m}$ from the source). Results shown for octave bands 250,500 and $1 \mathrm{k} \mathrm{Hz}$. The thick black line represents the smoothed ETC with time integration $20 \mathrm{~ms}$. Red line: average decay over $30 \mathrm{~dB}$. Green line: Early decay over $10 \mathrm{~dB}$ (EDT). Thin black line: backward integrated Schröder curve. Also indicated is the echo criterion EK according to [Dietsch86] (see par.3.5) for music/speech.

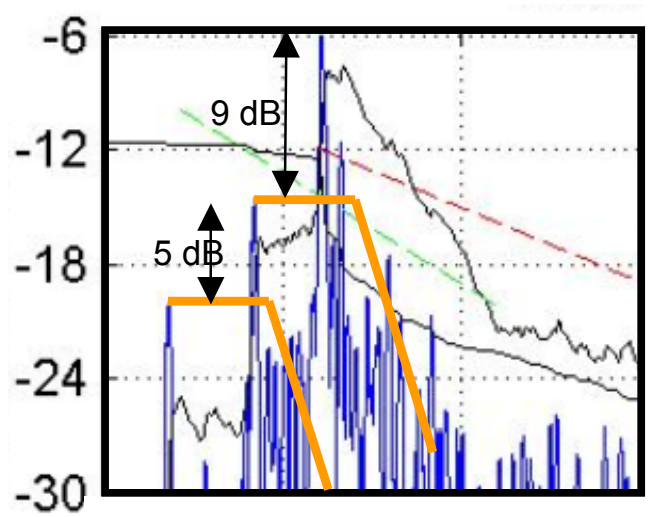

Figure 14.20. Analysis of the measured impulse response for the $500 \mathrm{~Hz}$ octave band with the method proposed by Yamamoto (see par 3.4)

\subsubsection{First proposed solution to solve the problem: diffusion}

To solve the problem either the sound must be prevented from reaching the dome or the reflection should be prevented from focusing.

The solution applied in Royal Albert Hall (see par. 14.4) was to prevent the sound from reaching the dome by using suspended reflectors. This concept could also be used to solve the echo in the Tonhalle, but at the same time this solution would significantly reduce the effective volume of the hall, which is already at the lower boundary of what is needed for a symphonic concert hall.

Since the problem is essentially caused by the geometry, the geometry had to be changed, to prevent the focusing to occur. As can be seen in figure 14.15 there is some space in between the inner dome (wooden panels) and the outer dome (concrete), that could be used. The first solution proposed was to apply sound diffusion elements attached to the outer dome, inspired by the diffusers of the former Nürnberger planetarium (figure 13.2, upper left).

The Tonhalle has a historical monument status and it was agreed that the wooden panels could only be replaced by an acoustically transparent wire mesh, if its size and position were identical to the wooden panels (see also [Vercammen06] and [Lorenz06]).

At that time the possibility of small suspended reflectors was also considered, to add early reflections (see figure 14.21). These reflectors have not been realized. 


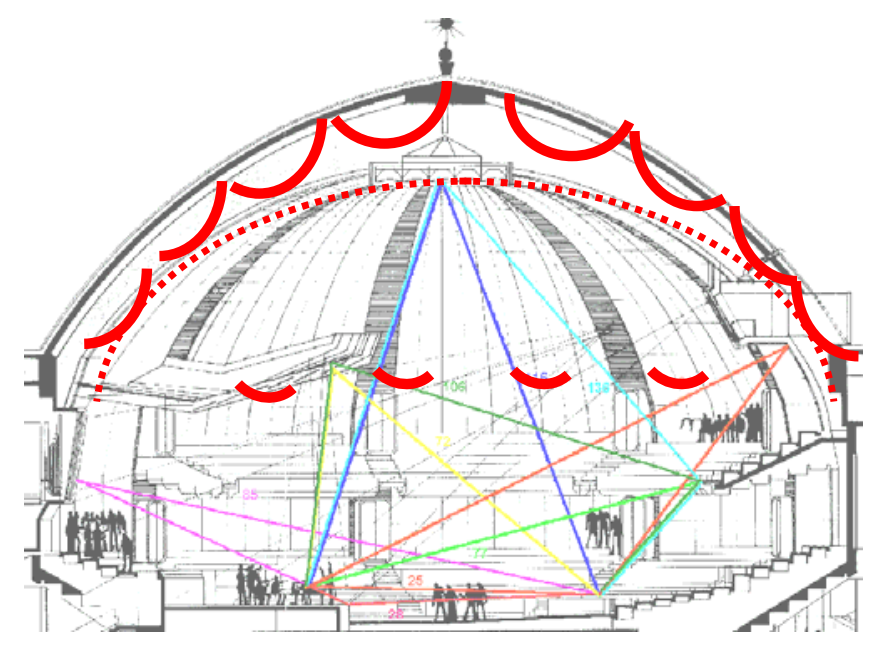

Figure 14.21. Schematic drawing of the first proposal to solve the echo by installing diffusers between the inner and outer dome, forming an acoustically transparent inner dome. Also showing: free suspended reflectors which were not realised.

\subsubsection{Scale model research of the first proposal: diffusion}

\section{Situation before renovation}

In 2004 an acoustical scale model research (1:12) had been done to test the effect of the proposed concept. The model was made of mdf (Medium-Density Fibreboard) and solid polystyreen. The measurement techniques are discussed in par. 14.2. As a reference the existing situation was modeled (see figure 14.22).
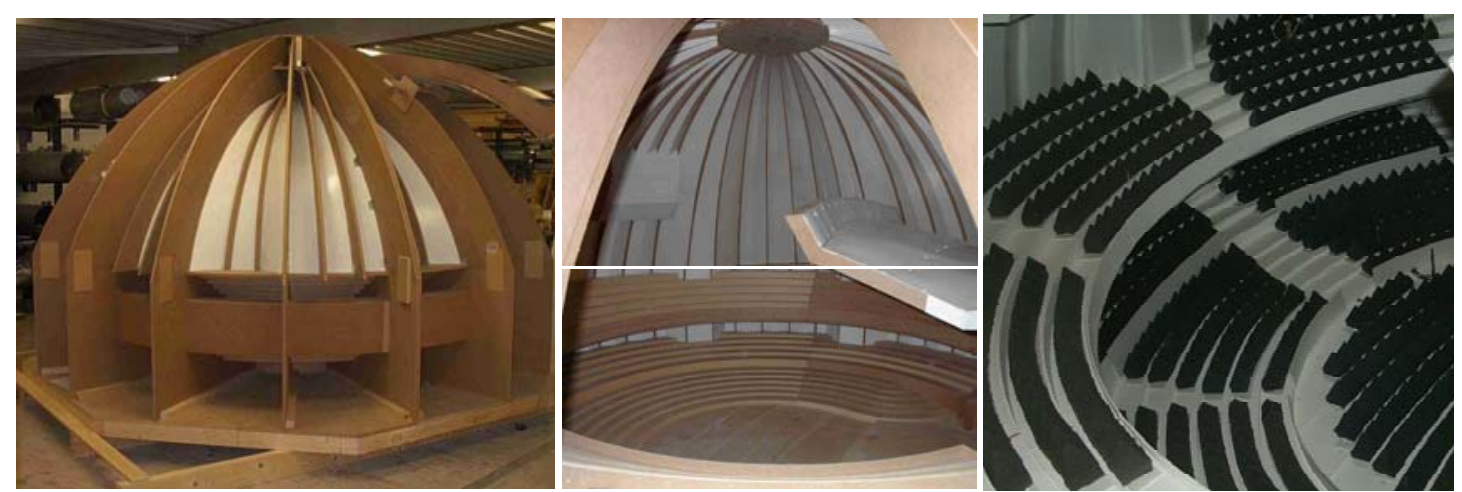

Figure 14.22. Scale model of the Tonhalle Düsseldorf. Left: outside; middle: inside, situation before renovation, during construction; right: after painting and with audience

The measured impulse response in the model was compared with the measurements in the real hall (with curtains over the seats to simulate the audience). The results at one measurement position, close to the centre of the hall, are shown in figure 14.23. This figure shows the measured impulse reponse as ETC (Energy Time Curve), as well as the integrated curve with a integration time constant of $20 \mathrm{~ms}$. Due to the integration of energy this line lies higher than the non-integrated ETC.

The results show that, though there might be differences in the absolute level of the reflected sound (which is also different at different measurement positions), the phenomenon of the dome reflection is found in the scale model. For the first measurements the scale model also showed a reflection at about $50 \mathrm{~ms}$. This was caused by the circular separating walls in the audience area and was reduced by raising the audience absorption. 

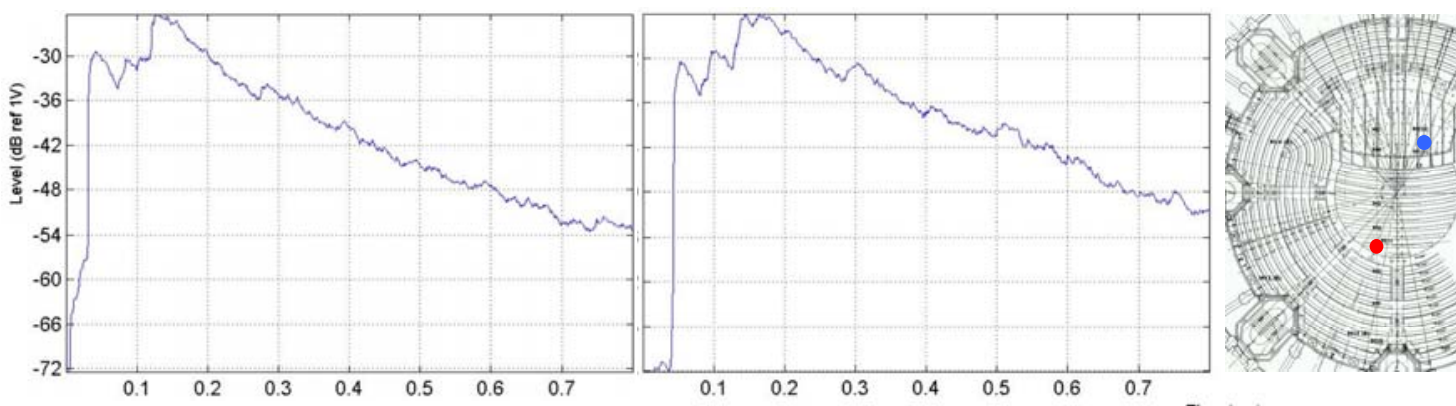

Figure 14.23. Measured impulse response (smoothed ETC) $1 \mathrm{kHz}$ octave band filtered in reality (left) and in the scale model (middle) before renovation, with audience (simulated in the real hall with curtains on seats). Right: indication of position of source (blue dot) and receiver (red dot)

\section{First proposal: diffusion}

Several types of diffusion, realistic and less realistic, were tested. One typical example is shown in figure 14.24. Although the strength of the reflection is reduced by a few decibels, it is still clearly visible. From listening to the impulses (transferred to scale 1:1) it was concluded that even if an separate echo could not be heard, there was an obvious coloration of the sound. As shown in paragraph 13.1 the reduction of the specular reflection by diffusing elements is limited and might be insufficient when the required reduction is more than $10 \mathrm{~dB}$. The existing structure is already diffusive to some extent, as can be concluded from figure 14.16. This limits the further reduction that can be achieved.

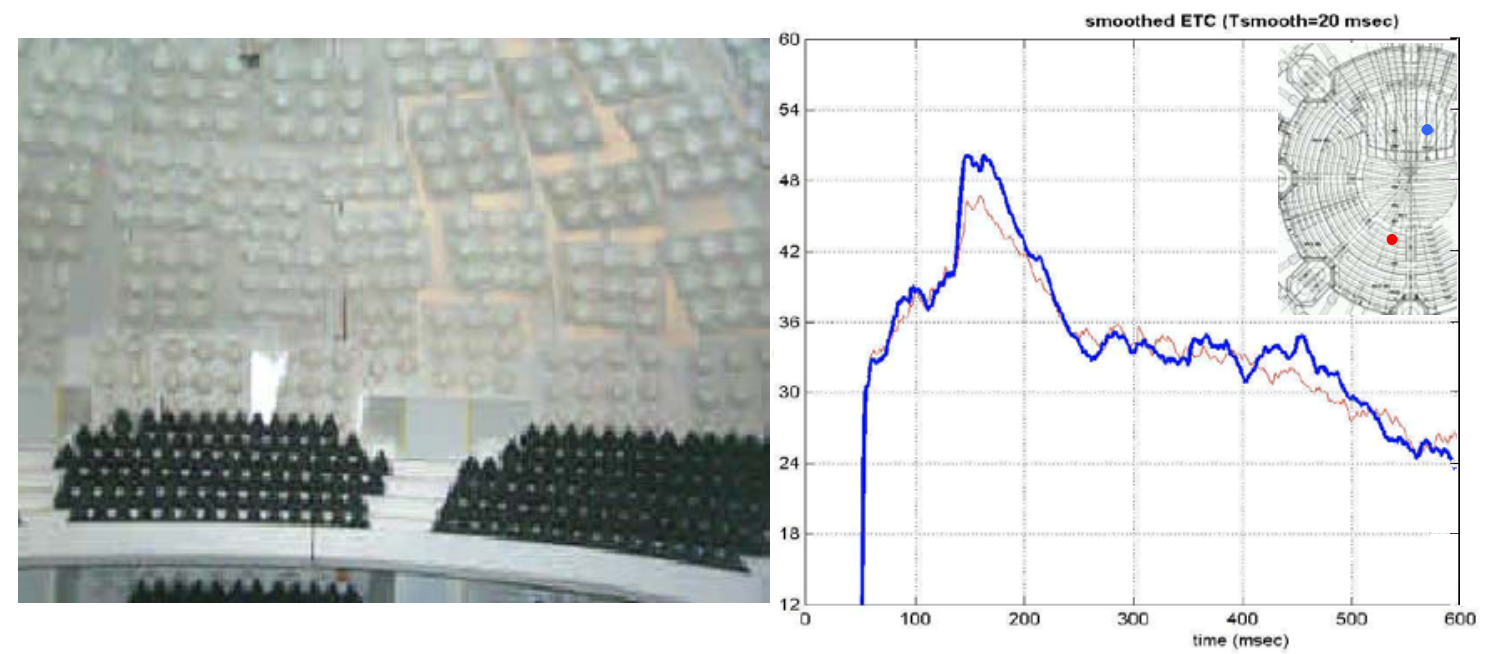

Figure 14.24. Scale model research Tonhalle Düsseldorf: one of the variants tested with diffusing elements. Left: Model with convex spherical diffusers; Right: Typical effect on the smoothed ETC. Blue: without diffusion elements; red with diffusers. Inset: indication of position of source (blue dot) and receiver (red dot)

\subsubsection{Second proposal: redirecting the sound}

A second proposal to solve the problem involved the concept of redirecting the sound as described in paragraph 13.4. Figure 14.25 (left) illustrates the principle of the reflecting panels, which had to be placed between the inner and outer dome. The panels are as large as could be placed between the inner and outer dome, typically between 2 and $3 \mathrm{~m}$. From figure 13.16 it can be derived that a size of 2 wavelengths is needed to achieve a 15 to $20 \mathrm{~dB}$ reduction at $30^{\circ}$. That means that these panels 
are sufficiently efficient for frequencies above 250 to $300 \mathrm{~Hz}$. This was regarded to be adequate.

As described in par. 13.4, the reflection pattern of the $30^{\circ}$ prisms was tested in an anechoic room. These measurements confirmed that for a suppression of the specular reflection of more than $10 \mathrm{~dB}$, the size of at least 2 wavelengths is needed.

Creating zigzag lines in the section would result in "rings" with cylinder and cone segments. Although the first reflection from these surfaces would be away from the dome-focusing point, it would create the risk of multiple clustered reflections and an irregular sound decay. To limit that risk a zigzag orientation in the horizontal section was proposed. The panels in the upper part of the dome were tilted in a fan shaped way. The resulting complicated 3D structure is shown in figure 14.25 (right).
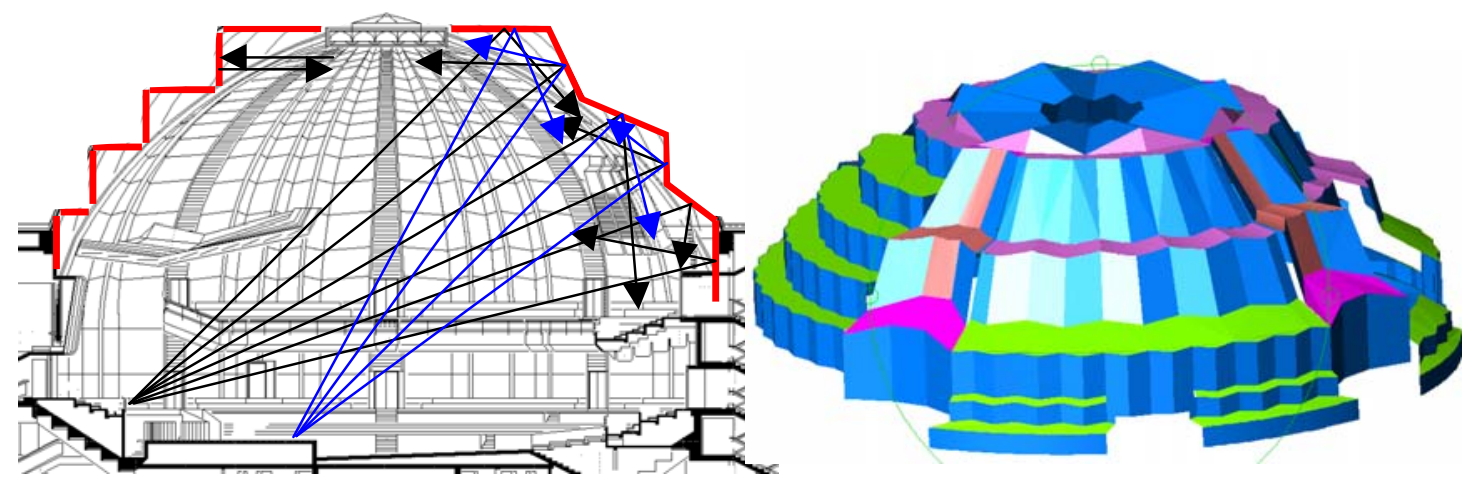

Figure 14.25. Renovation concept of the Tonhalle Düsseldorf: redirecting the reflections, either directly down towards the audience or up into the dome. Left: section, Right: $3 D$ visualisation of the new dome geometry.

\subsubsection{Scale model results with redirection of sound}

The next step was to test the second proposal with the redirection of sound in the scale model. Figure 14.26 shows a picture of the model as tested and the measured impulse response close to the echo position.
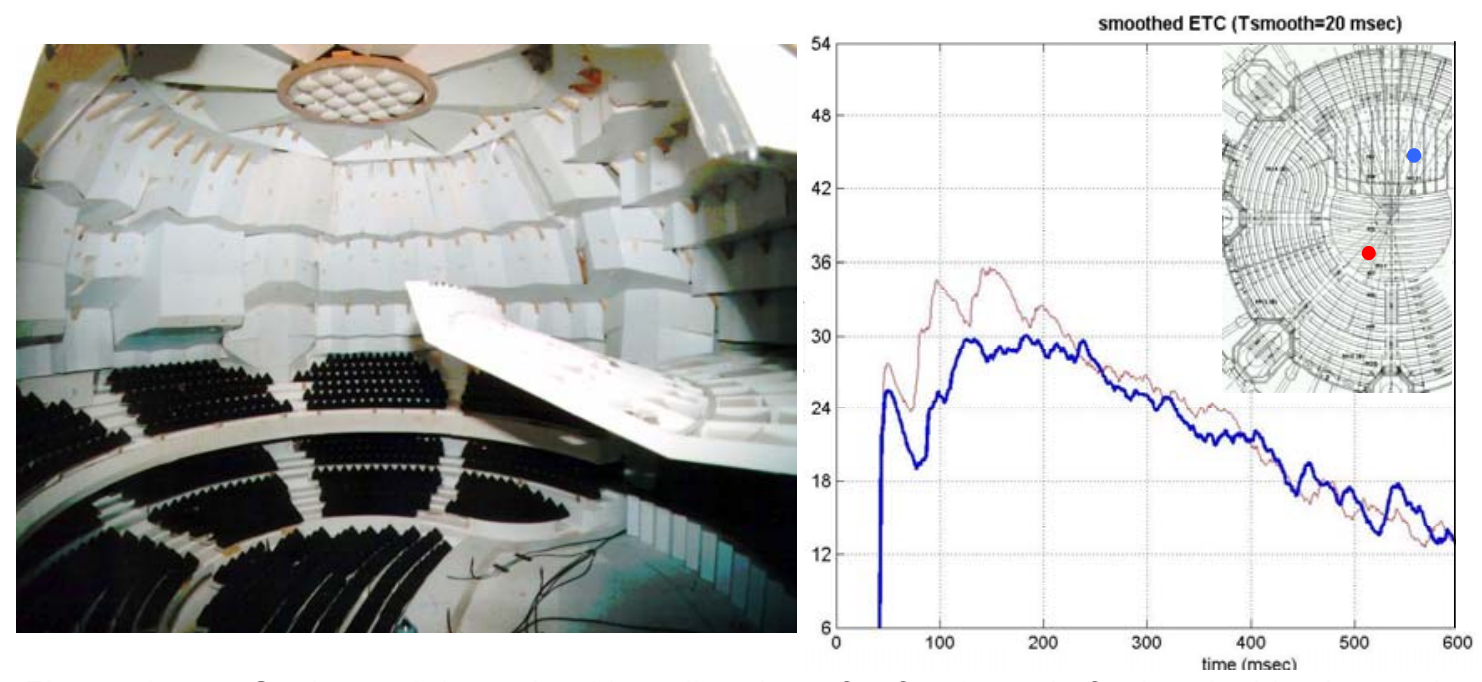

Figure 14.26. Scale model result with redirection of reflections: Left view inside the scale model with the reflecting panels as shown in figure 14.25; Right: smoothed ETC of hall before renovation (red) and with the redirecting panels (blue); Inset: source (blue dot) and receiver (red dot) positions 
The measurement results show a significant reduction of the echo at $130 \mathrm{~ms}$. The dome reflection is no longer visible.

As mentioned in par. 14.3.4, the earlier reflection (at this position around $100 \mathrm{~ms}$ ) is caused by the cylindrical parapet and was removed by lifting the audience area.

Based on the results of the scale model research it was decided to renovate the hall using this solution concept.

An important part of this concept, however, was to apply an acoustically transparent, visually "closed" structure. A wire mesh was applied that was tightened on a tension grid. Since this construction is in the same position as the wooden panels that had caused the echo, reflections from this new structure imposed a risk. The structure was tested for sound transparency and reflection strength in the anechoic chamber. The requirement was that the reflection should be $20 \mathrm{~dB}$ lower than the reflection from a flat panel. This was achieved after optimizing the supporting structure (circular instead of square steel profiles), as discussed in par. 13.3.

\subsubsection{Measurement results after renovation}

In the summer of 2005 the hall was renovated. The measured impulse response after renovation is shown in figure 14.27 , at the former echo position.

The level of the reflections at 250 and $500 \mathrm{~Hz}$ are about $6 \mathrm{~dB}$ under the direct level and are equal to the other reflections in the hall. The level of the dome reflection is reduced by ca. $20 \mathrm{~dB}$. The Dietsch and Kraak criterion fulfills the recommended value of 0,9 for speech.

\subsubsection{Conclusions}

Before renovation the Tonhalle Düsseldorf had a strong echo due to the focusing effect of the dome construction. Although some diffusion in the shape of the panels was realized, the echo was about $15 \mathrm{~dB}$ stronger than the direct sound. Numerical calculations based on the Kirchhoff integral confirmed the order of magnitude of this amplification.

Scale model research was used to verify the efficiency of several proposals to reduce the echo. A concept that used diffusers was able to reduce the echo, but not sufficiently, since the sound reduction potential of diffusers is limited to about $10 \mathrm{~dB}$.

A second proposal that used redirecting panels showed a very promising result in the scale model: the echo disappeared. The tested concept with redirecting panels and an acoustically transparent wire mesh replacing the former wooden panels was realised in the real hall. Measurements indicated the removal of the echo. 


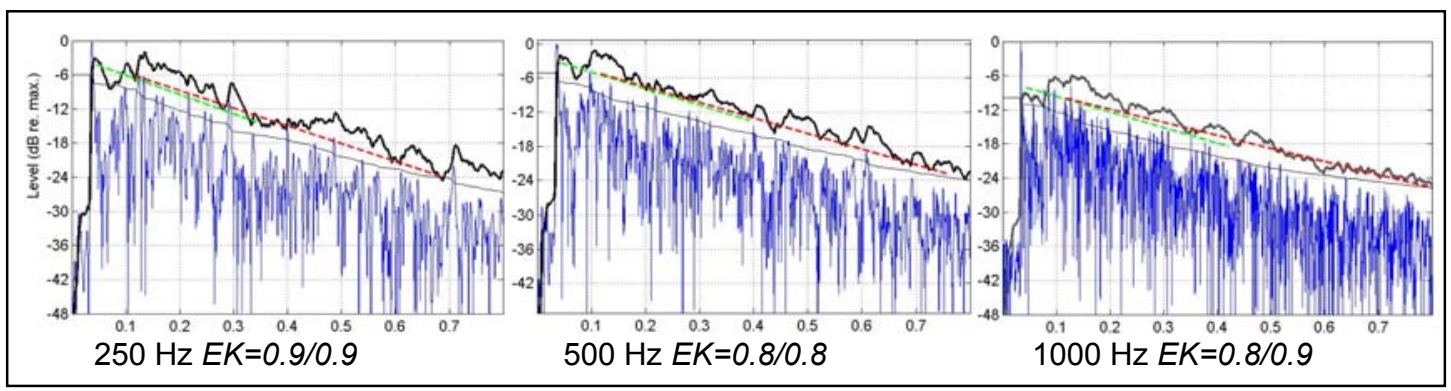

Figure 14.27. Measured impulse response ETC in the Tonhalle Düsseldorf after renovation, without audience, at the former echo position (receiver at ca. 10,5 $\mathrm{m}$ from the source). Results are shown for octave bands 250,500 and $1 \mathrm{k} \mathrm{Hz}$. The thick black line represents the smoothed ETC with time integration $20 \mathrm{~ms}$. Red line: average decay over $30 \mathrm{~dB}$. Green line: Early decay over $10 \mathrm{~dB}$ (EDT). Thin black line: backward integrated Schröder curve. Also indicated is the echo criterion EK according to [Dietsch86] (see par.3.5) for music/speech.

The first opening concerts (with percussion and piano!) convinced the audience of the removal. In addition to the removal of the echo, several other room acoustical improvements have been realized, such as an enhancement of the reflections on stage and a longer reverberation time. The acoustical requirement of the acoustically open inner dome gave rise to a new architecture for this hall, see figure 14.28. All alterations together made this renovation a significant success.

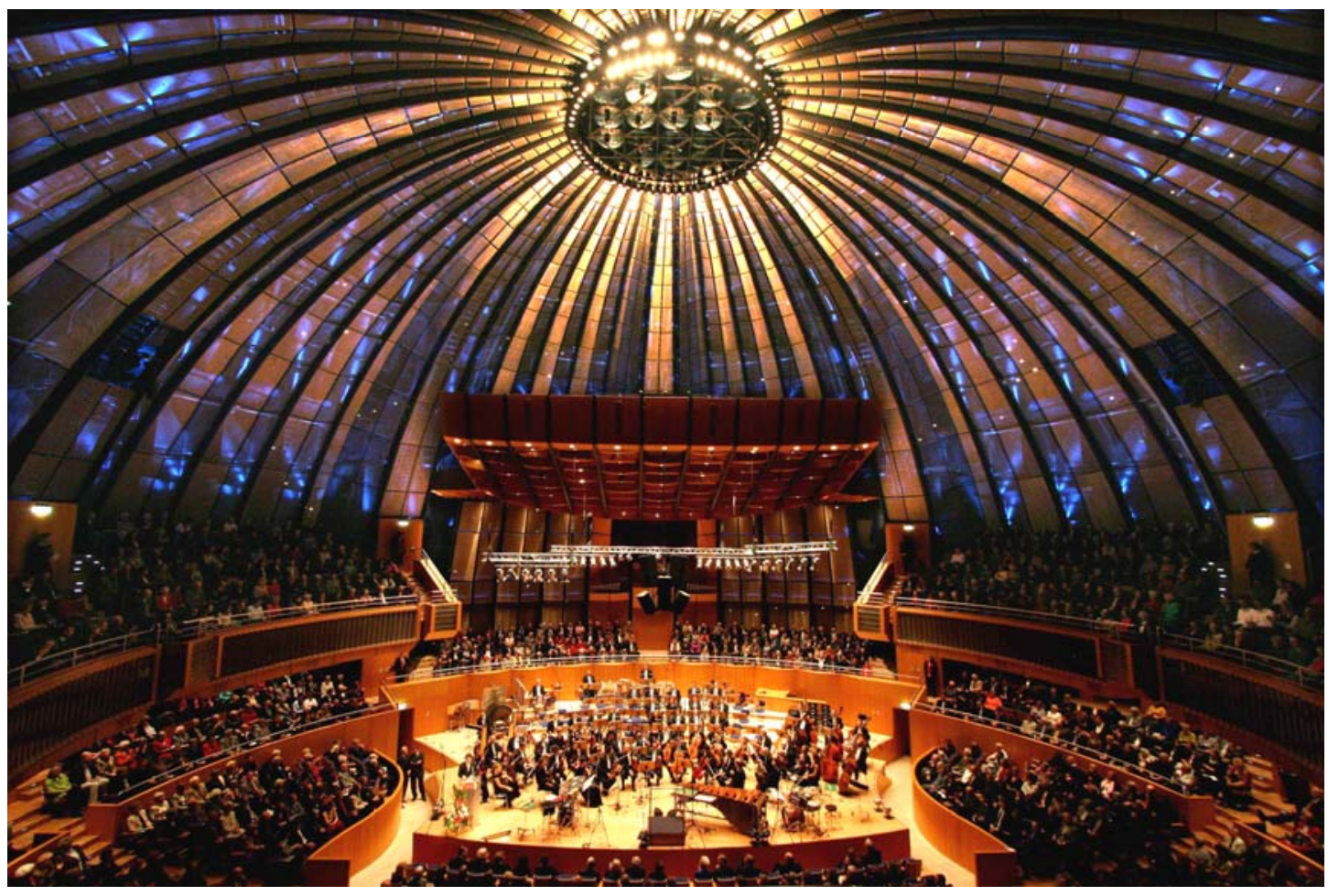

Figure 14.28. Interior of the Tonhalle Düsseldorf after the 2005 renovation. 


\subsection{Royal Albert Hall London}

The Royal Albert Hall, see figure 14.29, is London's premier venue for a wide range of events: symphonic music (The Proms season), organ and choral performances, large meetings, rock concerts, sports events and more. Because of its large size (approx. $80.000 \mathrm{~m}^{3}$, ca. 5000 seats) and its quasi-ellipsoidal (dome) shape it has suffered from several echo problems since the day it was opened. [Metkemeijer02]. The acoustic problem of the hall is described in many books (e.g., [Bagenal31], [Izenour77], [Cremer78], see also [Bagenal41], [Nat.Phys28]).

In 1968 convex spherical elements (called "flying saucers" or "mushrooms") solved the problem to some extent.
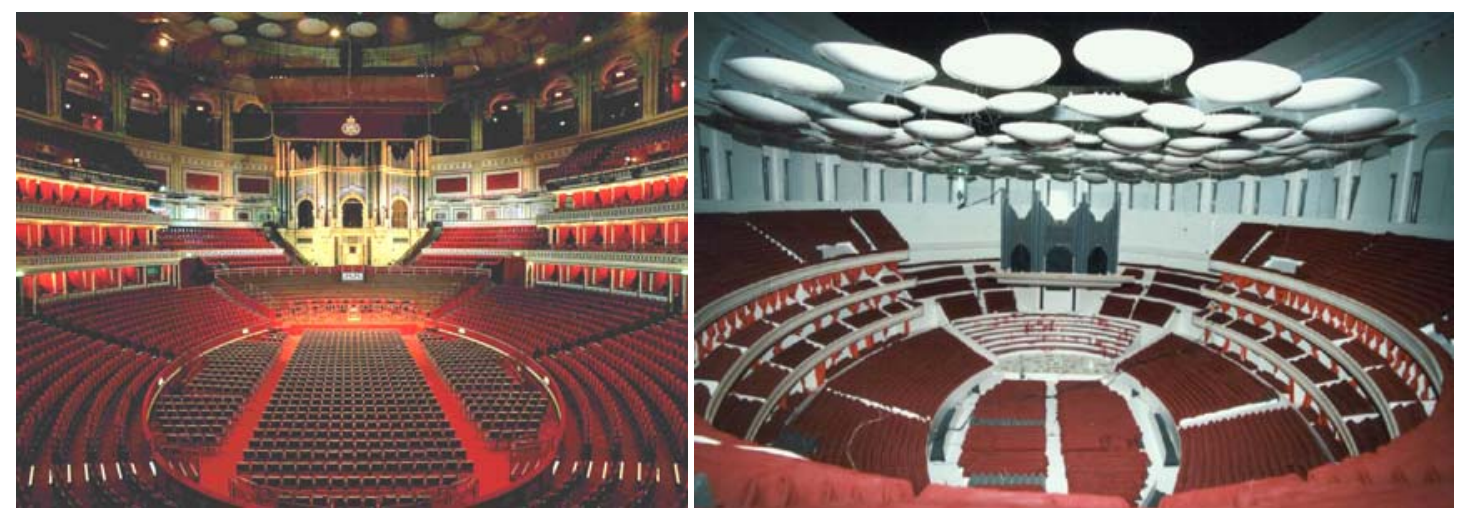

Figure 14.29. Left: Interior of the Royal Albert Hall, Right: Scale model 1:12, showing the flying saucers.

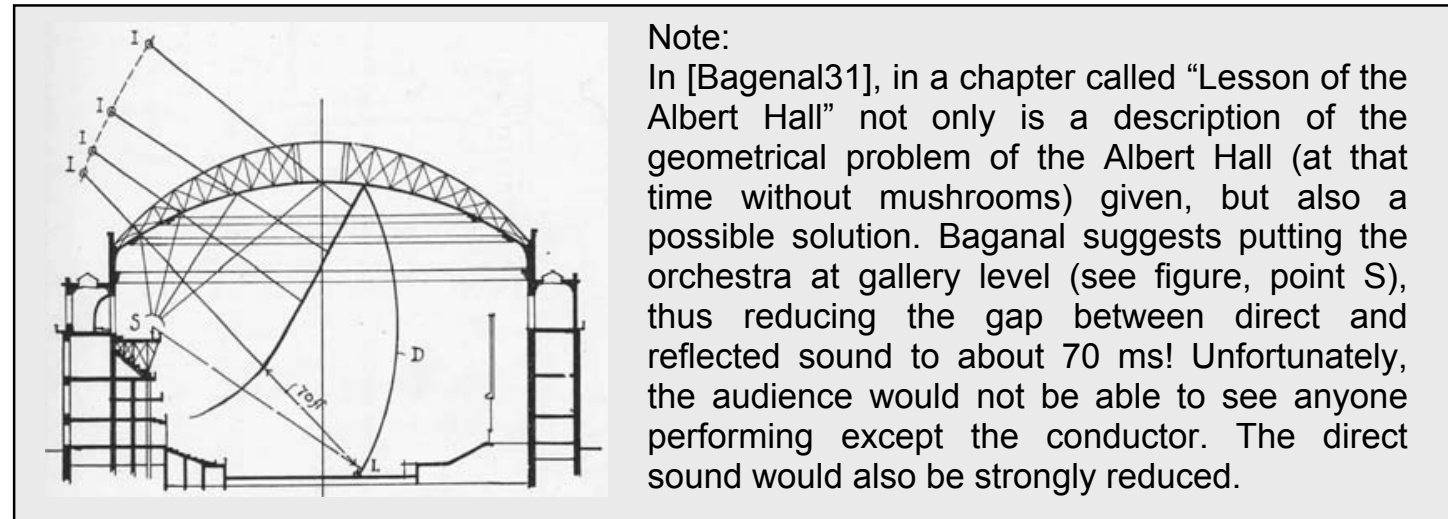

\subsubsection{Geometrical approximation}

The hall has an elliptical floor plan and it increases in size at higher levels. The roof has an ellipsoidal shape. Figure 14.30 shows a longitudinal section of the hall. The basic prolate ellipsoid has dimensions of (approximately) $a=45 \mathrm{~m}, b=45 \mathrm{~m}, \mathrm{c}=55 \mathrm{~m}$. The two focal points are about $31.6 \mathrm{~m}$ from the centre and $6 \mathrm{~m}$ under stage level.

The distance $z_{f}$ from the focal point to the intersection point of the wave approximation, with the geometrical field along the reflection axis (12.1), will be about $4 \mathrm{~m}$ at $250 \mathrm{~Hz}$.

This means that there is not a full focusing effect to be expected for frequencies of $250 \mathrm{~Hz}$ and higher and thus the sound concentration can be evaluated with a geometrical approach. 


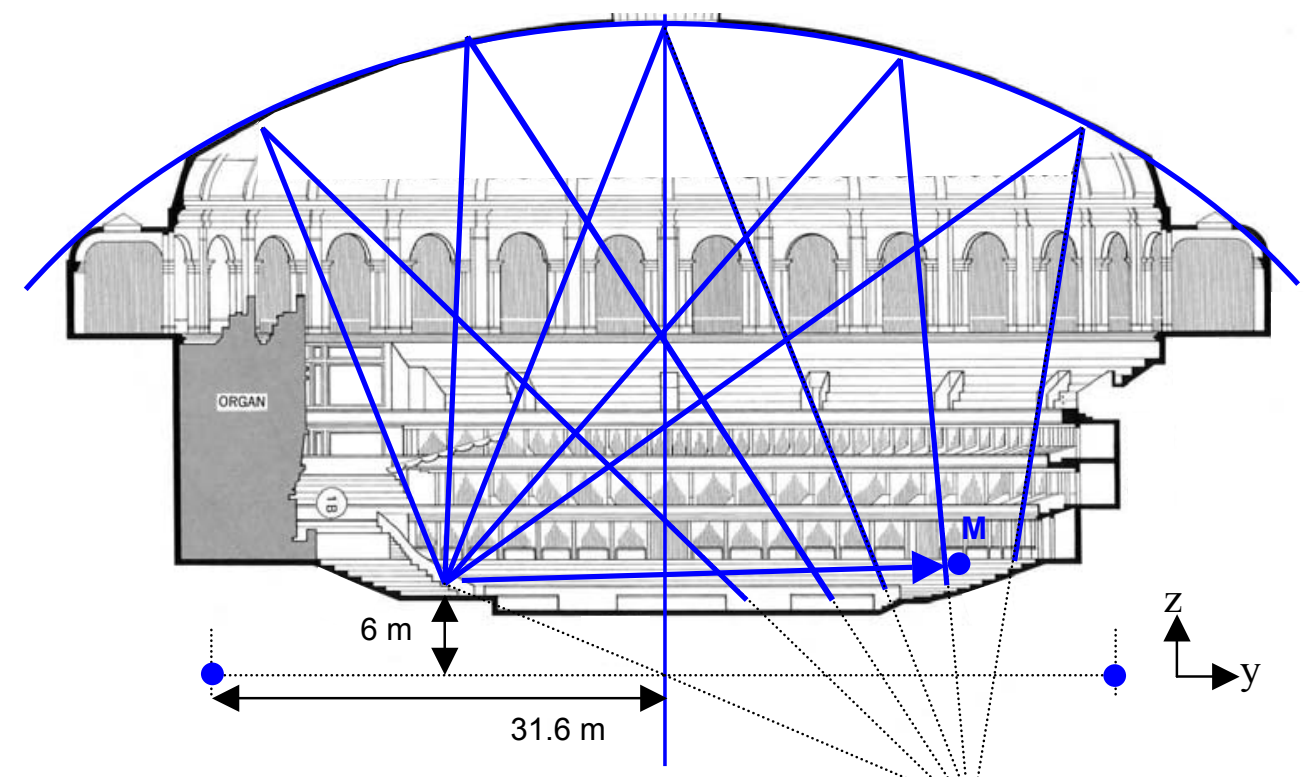

Figure 14.30. Longitudinal section of the Royal Albert Hall with the ellipsoidal shape indicated, as well as the focal points and the reflected sound field from the dome. Measuring position $M$ is indicated.

In the first approximation the mushrooms will not be considered. From formula (5.16) it is calculated (see below) that for a position at $42 \mathrm{~m}$ from the source, the level of the reflection will be approx. $8,5 \mathrm{~dB}$ above direct sound. Since this is a purely geometrical approach, the increase is basically frequency independent.

At position $\mathrm{M}$ the delay of the reflected sound (at approx. $260 \mathrm{~ms}$ ) will be about 130 $\mathrm{ms}$ after the direct sound (at $130 \mathrm{~ms}$ ). The threshold of audibility of a single reflection at $130 \mathrm{~ms}$ is around $\Delta L=-14 d B$ (with $\Delta L$ relative to the direct signal) for fast violin music (see line 2 in Figure 3.5). At position $M$ this threshold is exceeded by $23 \mathrm{~dB}$.

For a concert hall, however, this evaluation is not very realistic, since there are many reflections and the specific reflection from the dome can only be audible when it can be heard above the other reflections from walls etc.

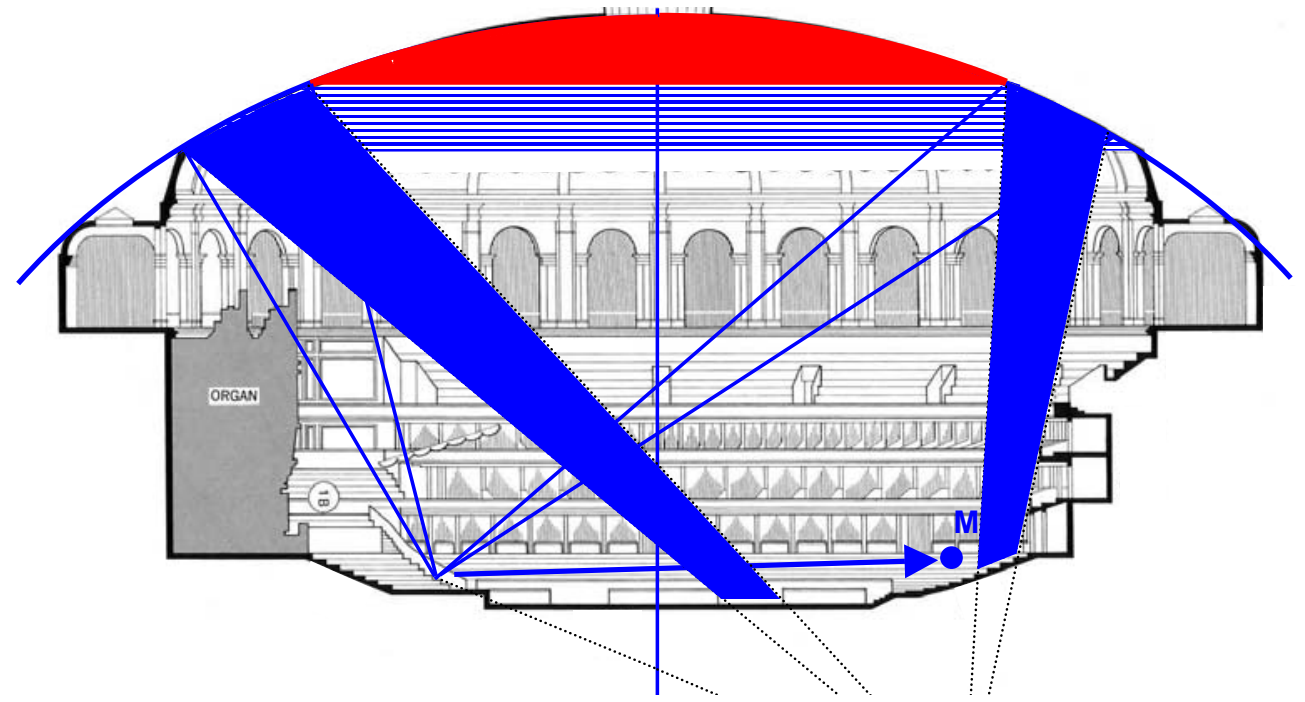

Figure 14.31. Geometrical reflection from the upper cove. Middle (red) part of the roof is sound absorbing. 
Due to its large volume and absorption, the reverberant sound level is very low in the Royal Albert Hall. Even individual reflections from hard flat surfaces, might be above reverberance level and can be individually audible.

It can be expected that the reflection from the ceiling, due to its ellipsoidal shape, will be appr. $\Delta L_{c} \approx 15 \mathrm{~dB}$ stronger than reflections from hard, flat surfaces (see note).

Note:

The geometrical decrease of the reflected sound level is calculated here for position $M$, at $42 \mathrm{~m}$ from the source. The direct SPL at this position will be:

$$
L p_{d}=L p_{1 m}-20 \log (42)=L p_{1 m}-32,5 \mathrm{~dB}
$$

Both the distance $s$ from the source and the distance $d$ from point $\mathrm{M}$ to the centre of the dome are about $42 \mathrm{~m}$ in $z$-direction and $39 \mathrm{~m}$ in $x$-direction.

If the reflection would take place against a flat surface, the level decrease (relative to $1 \mathrm{~m}$ from the source) would be $-20 \lg (84)=-38,5 \mathrm{~dB}$.

The curved ceiling causes an amplification that can be calculated from (5.16):

$$
\Delta L_{c}=-10 \lg \left(\left|\frac{1}{\left(\frac{1}{d_{x}}+\frac{1}{s_{x}}\right)} \frac{2}{R_{x} \cos \theta_{x}}-1\right|\right)-10 \lg \left(\left|\frac{1}{\left(\frac{1}{d_{z}}+\frac{1}{s_{z}}\right)} \frac{2}{R_{z} \cos \theta_{z}}-1\right|\right)
$$

The radius of curvature for the reflection point on the roof in the longitudinal section, reflecting to position $\mathrm{M}$, can be calculated with the method described in paragraph 5.2.3. The radius at this point will be approx. $R_{z}=62 \mathrm{~m}$

with $R_{x}=45 \mathrm{~m}, \cos \theta_{x}=1, \cos \theta_{z}=0,93$ this results in

$$
\Delta L_{c}=14,5 \mathrm{~dB}
$$

so the reflection will be approx.

$$
L p_{r}=L p_{1 m}-38,5+14,5=L p_{1 m}-24 \mathrm{~dB} .
$$

At position $M$ the level of the reflection $L p_{r}$ will be about $-24+32,5=8,5 \mathrm{~dB}$ above the direct sound $L p_{d}$.

In this second step, the effect of the sound absorbing ceiling and the mushrooms may be considered.

The absorption is in the middle of the dome. Without mushrooms only the upper cove will reflect, generating a ring of reflections in the stalls (see figure 14.31). In the lightened area the pressure according to (5.16) will remain the same, since outside the focal point the amplification is not dependent on the reflecting surface area.

In the shaded area in the middle the sound pressure will be reduced according to the absorption factor of the material.

Due to diffraction the differences between lightened and shaded zones will be smaller.

The high frequency effect of the mushrooms will be proportional to their surface area. Since the wave will pass the array of mushrooms twice, the effect will be double. Therefore, with a density of $50 \%$, a reduction of $6 \mathrm{~dB}$ is to be expected. 


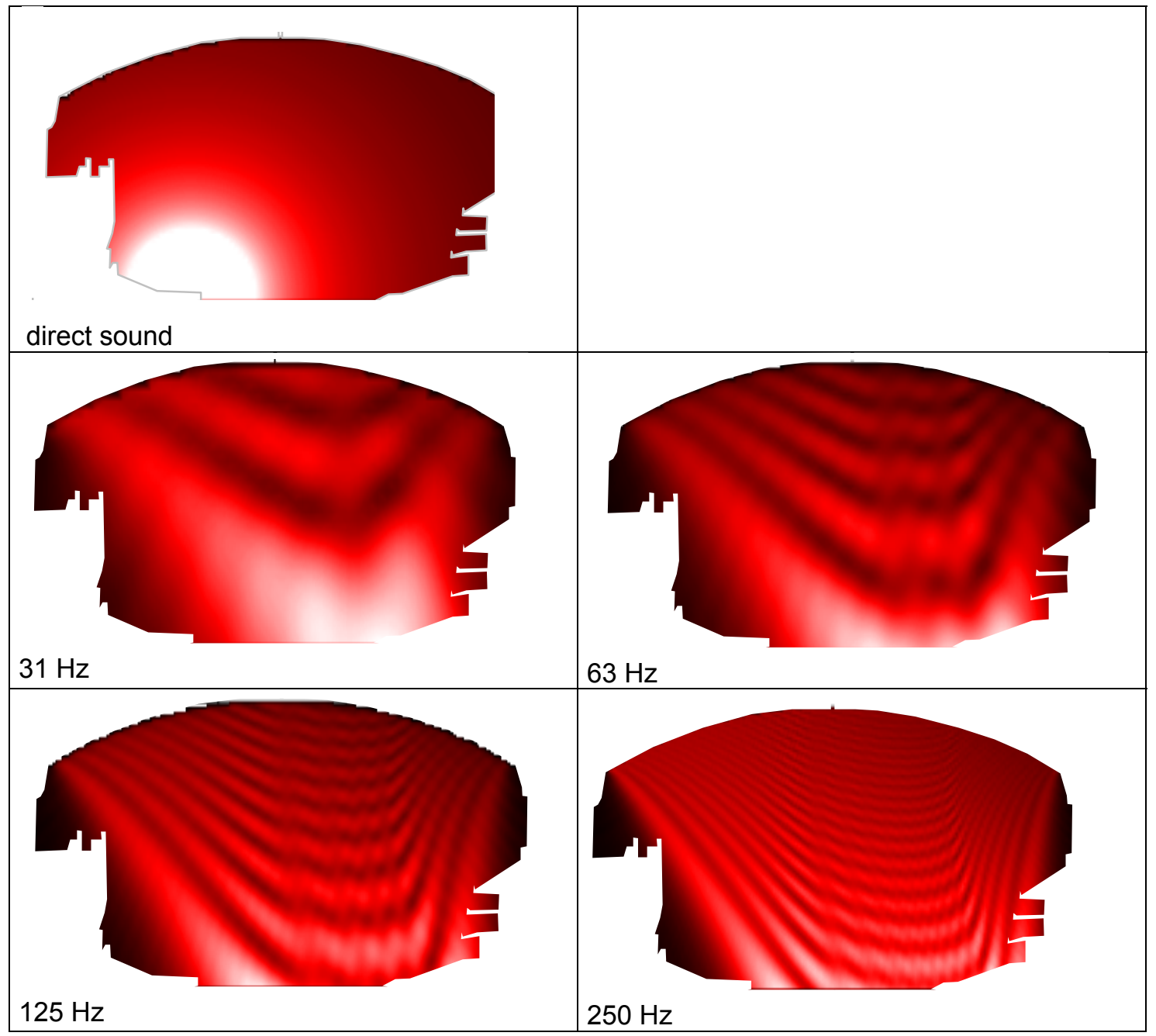

Figure 14.32. Numerical calculations according to (6.19) of the sound field in the Royal Albert Hall, without stage reflector, without mushrooms and with fully reflecting dome. Shown is the Longitudinal cross section with contour of the hall superimposed over the calculation result. Upper left: direct sound pressure from the source with $\hat{p}=1 \mathrm{~N} / \mathrm{m}$. Other: (single) reflected sound pressure from the ellipsoidal dome for the pure tone frequencies $31 \mathrm{~Hz}, 63 \mathrm{~Hz}, 125 \mathrm{~Hz}$ and $250 \mathrm{~Hz}$. The color indicates the pressure : White: $\geq 0,1 \mathrm{~Pa}(-20 \mathrm{~dB})$, black: $0 \mathrm{~Pa}$.

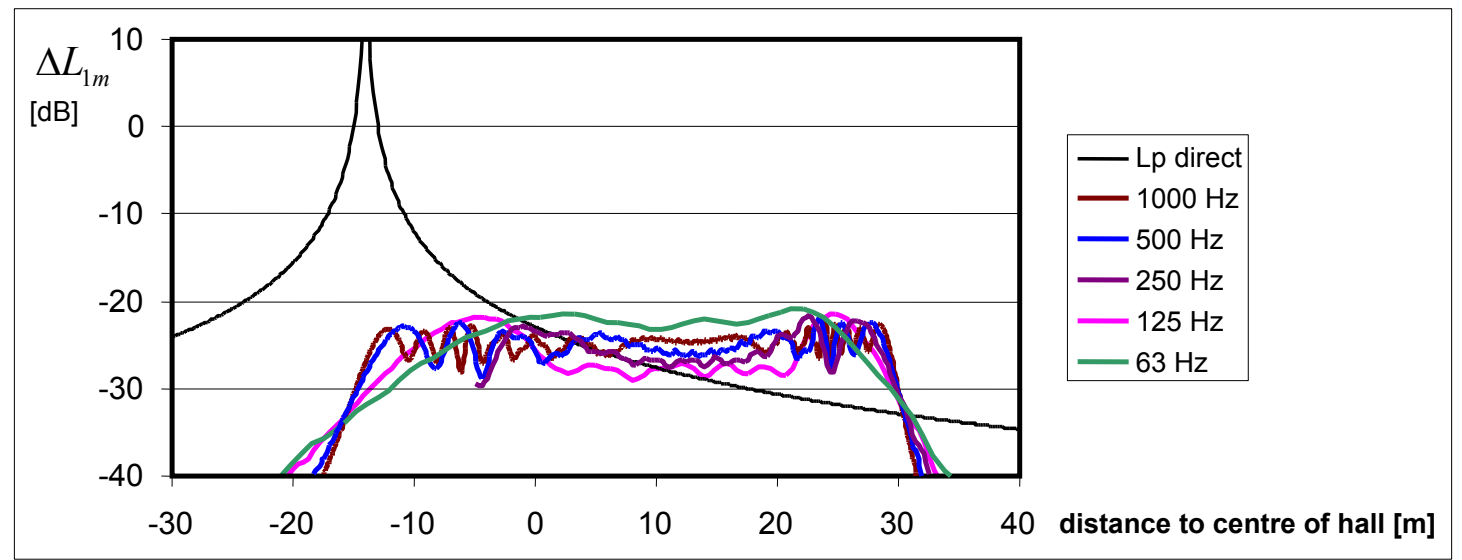

Figure 14.33. The $\Delta L_{1 m}$ (SPL relative to the $S P L$ at $1 \mathrm{~m}$ from the source) due to the reflection from the ellipsoidal roof of the Royal Albert Hall, calculated using (6.19), as a function of the distance from the centre, over the length of the hall (in cross section in the middle, at stage height). The monopole source is $14 \mathrm{~m}$ from the centre. Results are for several (pure tone) frequencies. Also shown: SPL of the direct sound. 


\subsubsection{Numerical approximation}

The SPL can also be calculated using Kirchhoff's integral for the situation with only the reflection from the ellipsoidal roof. The numerical solution with (6.19), using the normal to the surface according to (9.2), is shown in figures 14.32 and 14.33 . Figure 14.32 presents the pressure in the longitudinal section of the hall for the frequencies $31.5,63,125 \mathrm{~Hz}$ and $250 \mathrm{~Hz}$.

In figure 14.33 the SPL, relative to the SPL at $1 \mathrm{~m}$ from the source, is presented along a horizontal line, which is $6 \mathrm{~m}$ above the centre of the ellipsoid (corresponding roughly to the stage level), along the length-axis of the hall.

The figure shows a clear geometrical reflection area that corresponds to the geometrical illuminated area.

All these calculations are done for pure tones and the frequencies correspond to the middle frequencies of the octave bands. The level varies with frequency and position, however, at approx. $25 \mathrm{~m}$ from the centre of the hall a maximum seems to occur for most frequencies. The maximum is around $-23 \mathrm{~dB}$ (relative to SPL at $1 \mathrm{~m}$ ), which corresponds to the result of the geometrical method.

\subsubsection{Scale model measurements}

As part of the renovation that lasted from 1997 to 2003 an extensive scale model research was performed by Peutz (see figure 14.29, right). One of the situations modelled was the situation without mushrooms and with a reflecting dome, which was the actual situation at opening in 1871.

Figure 14.34 (left) shows the measurement result at position $\mathrm{M}$ with the reflected sound from the dome at about 260 to $300 \mathrm{~ms}$, for this position starting at $130 \mathrm{~ms}$ after the direct sound.

The integrated sound level of the peak at 260 to $300 \mathrm{~ms}$ is $11 \mathrm{~dB}$ higher than the direct sound, which roughly corresponds to the expected increase of approx. $8,5 \mathrm{~dB}$ (par.14.4.1).

It can also be seen that the level of the peak is $15 \mathrm{~dB}$, or more, higher than the adjacent reflections.

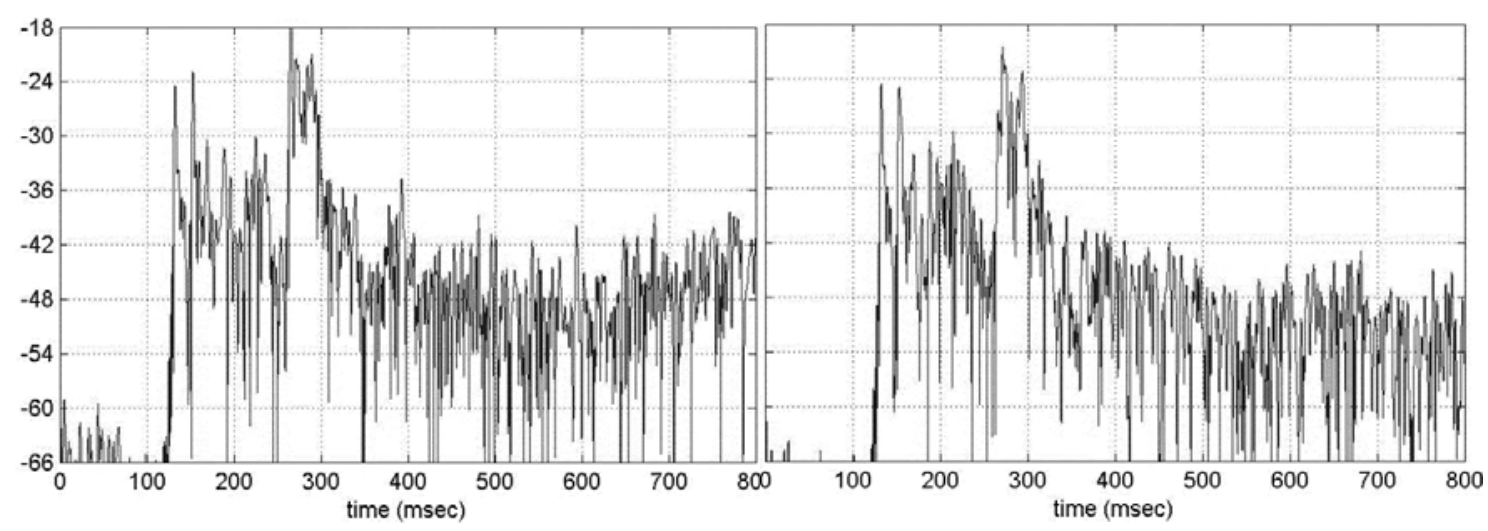

Figure 14.34. Measured ETC (Energy Time Curve) in the scale model of the Royal Albert Hall at position $\mathrm{M}$ for the $1 \mathrm{kHz}$ octave band, without mushrooms. Left: reflecting dome (situation of 1871) and right: with absorbing dome (situation of 1950) 
Shortly after opening, a velarium was hung under the glass roof. In 1941 the velarium was lowered to improve the acoustics for the Proms. In 1949 the glass part of the roof and the velarium were replaced by sound absorbing perforated aluminum. However, the part of the dome that was treated consisted of only about $50 \%$ of the total ceiling area. The surrounding cove remained reflective. The reduction at position $M$ is about $3 \mathrm{~dB}$ at $1 \mathrm{kHz}$ (see figure 14.34).

In 1968 the problem was solved to a great extent by installing the flying saucers. Figure 14.35 shows the analysis of the echo problem [Shearer70]. The roof construction consists of (a) an absorbing part (the perforated aluminum) that reduces the strength of the reflection in (b) the higher cove and (c) the lower cove (see figure 14.38). The curvature of the lower cove is sufficient to give diffuse reflections at some distance. The dome with the absorbing part, and especially the higher cove, project their reflection into the audience, as illustrated in figure 14.35 (in this graph full focusing in the audience area is suggested, however this is not the case, see figure 14.31).

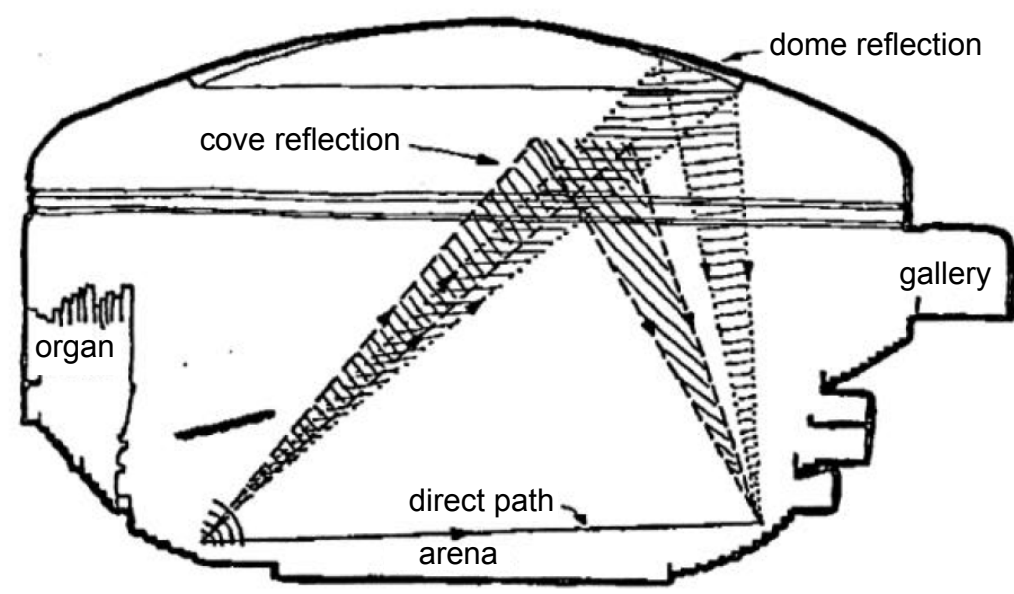

Figure 14.35. Echo analysis of the Royal Albert Hall by Shearer in 1968, longitudinal section [Shearer70]

Shearer's solution was to install suspended reflectors (the "mushrooms" or "flying saucers"), thus partly preventing the sound from reaching the dome and also providing early (or earlier) reflections. He tested the efficiency of the saucer arrangement in an anechoic room. Although there was a significant improvement, the echo did not completely disappear. Figure 14.36 (left) shows the remaining echo at position $\mathrm{M}$ in the scale model with the mushrooms (situation from 1968 until 1996). An additional reduction of $4 \mathrm{~dB}$ (relative to only absorption on the dome) has been achieved (position $\mathrm{M}, 1 \mathrm{kHz}$ ). 


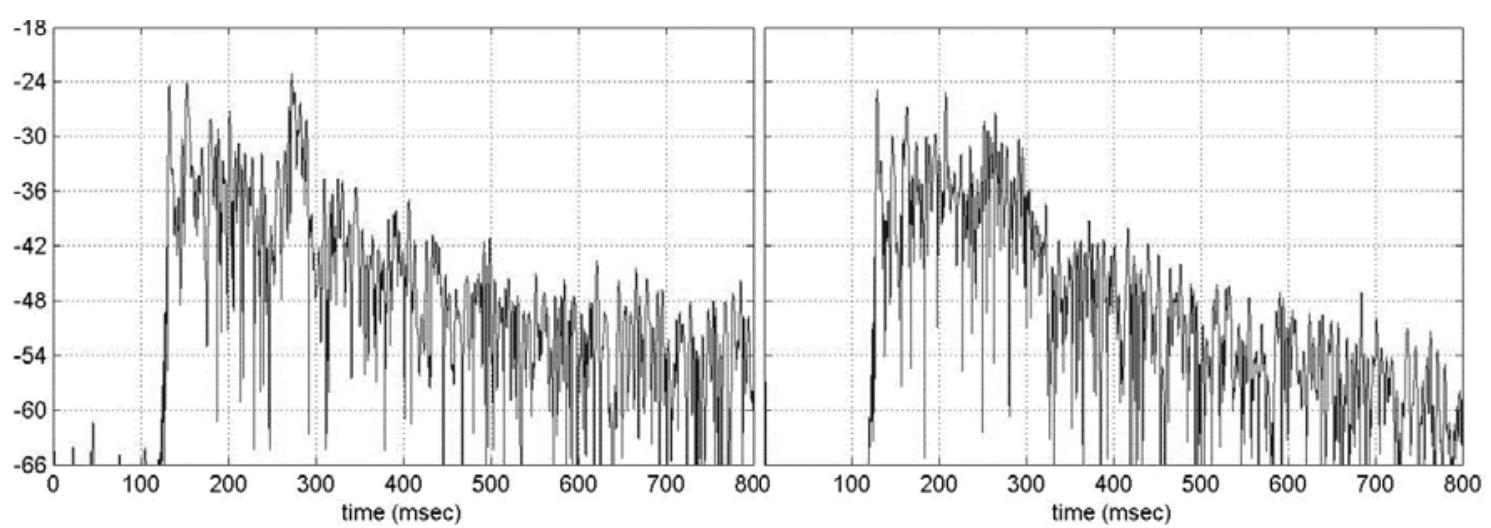

Figure 14.36. Measured ETC in the scale model of the Royal Albert Hall at position M for the $1 \mathrm{kHz}$ octave band, left: with mushrooms and canopy (situation from 1968 to 1996), right: with new condensed mushroom arrangement after renovation.

From 1997 until 2003 major refurbishment work took place. An important part of this operation was to improve the acoustic quality. Basic improvements were tested in the scale model shown in figure 14.29, e.g., bringing back the velarium as a convex sound reflector [Metkemeijer02]. Figure 14.37 shows the efficiency of such a solution in suppressing the echo. For reasons of heritage, however, the velarium was not realised.
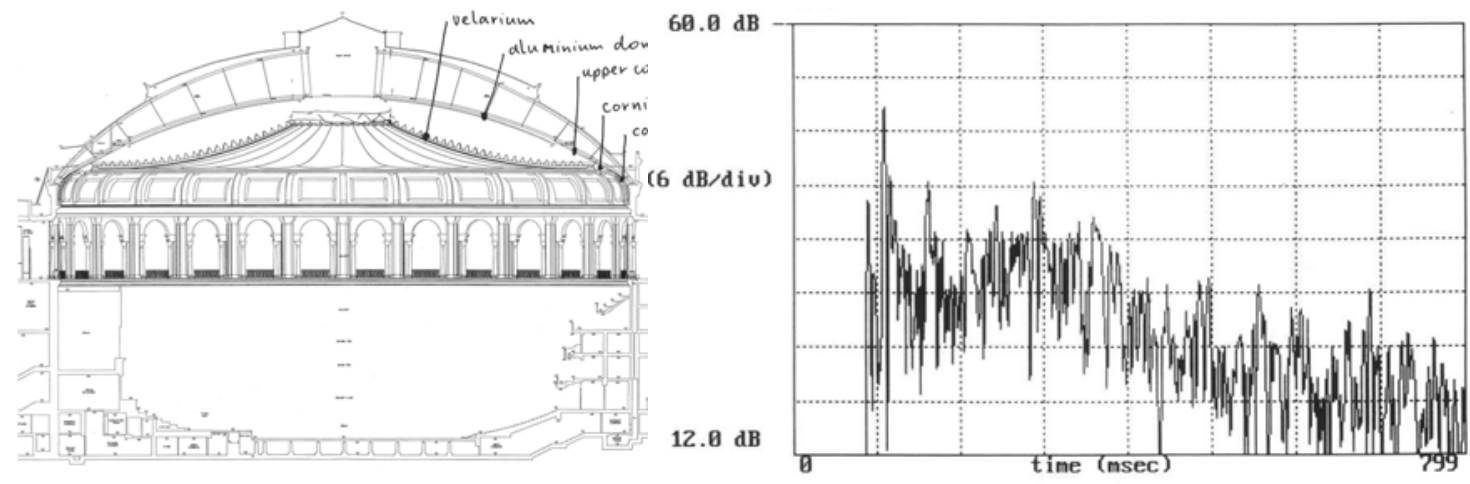

Figure 14.37. The suggested solution for the Royal Albert Hall, a convex velarium: left section with the velarium, right: measured ETC in the Royal Albert Hall scale model for the $1 \mathrm{kHz}$ octave band, with velarium (not realised).

Instead it was decided to rearrange the saucers, with a higher density for the higher cove and a better visibility of the (acoustically harmless) lower cove (see figure 14.38 and 14.39). 


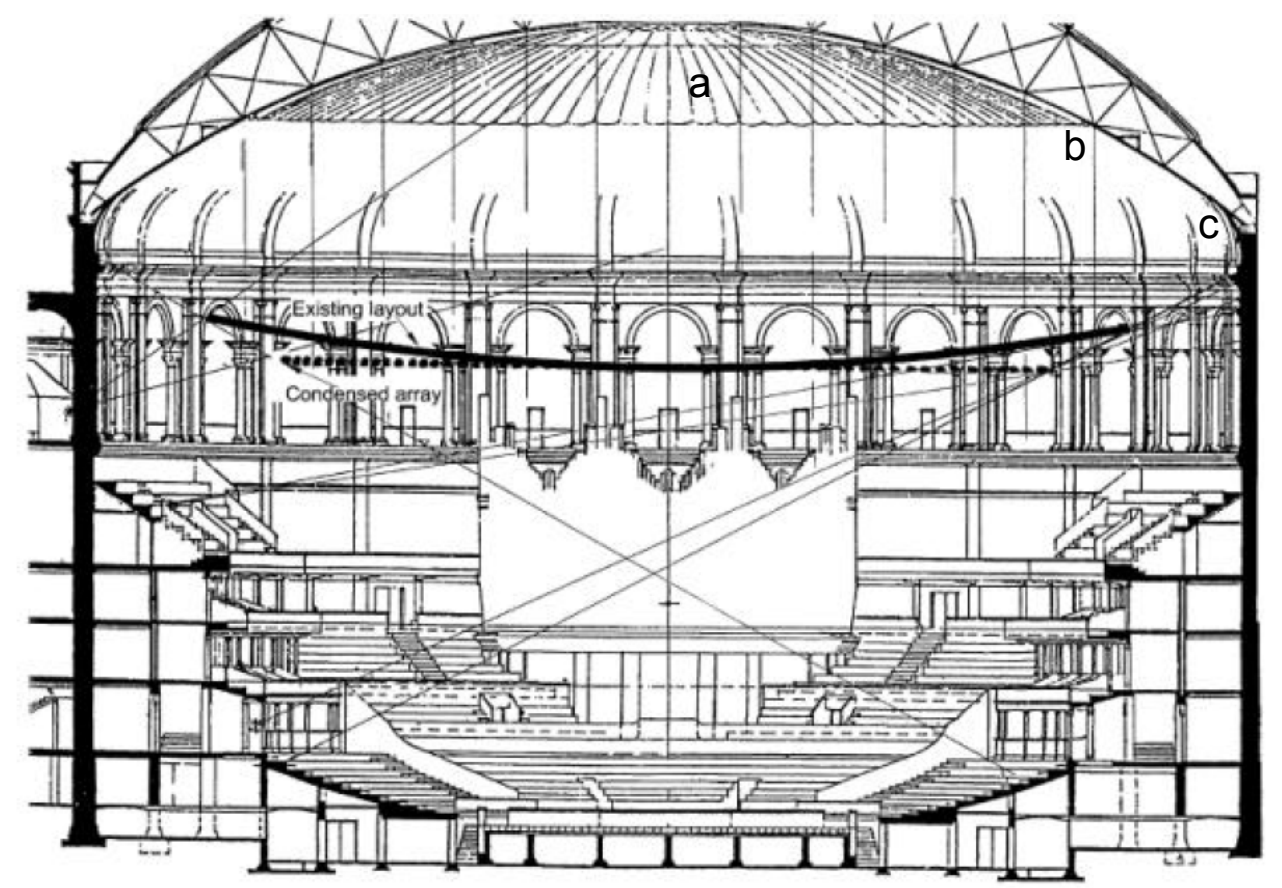

Figure 14.38. Cross section of the Royal Albert Hall. Shown are the positions of the old ("existing") layout of the flying saucers and the condensed layout that was applied in the 1996 refurbishment. a: Absorbing roof (perforated aluminum), b: higher cove, c: lower cove.

The measurement results show a further improvement of about $3 \mathrm{~dB}$ in the echo suppression due to the higher density of the mushrooms (see figure 14.36, right).
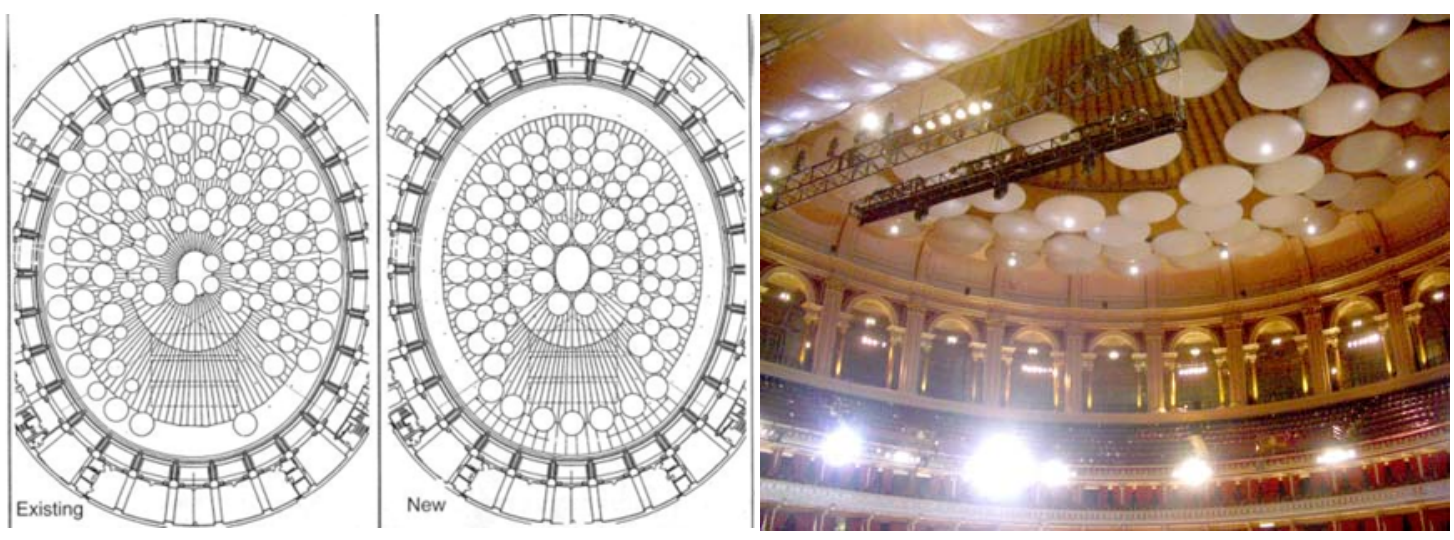

Figure 14.39. Plan showing the arrangement of flying saucers in the Royal Albert Hall before (left) and after (middle) renovation [Metkemeijer02]. The picture (right) shows how the lines of sight to the high cove are interrupted by the saucers (after renovation). 


\subsubsection{Real scale measurements}

Impulse response measurements were performed in the real hall before and after renovation. Figure 14.40 shows the results for the measurement position $M$ (the same as the shown in the previous paragraph).

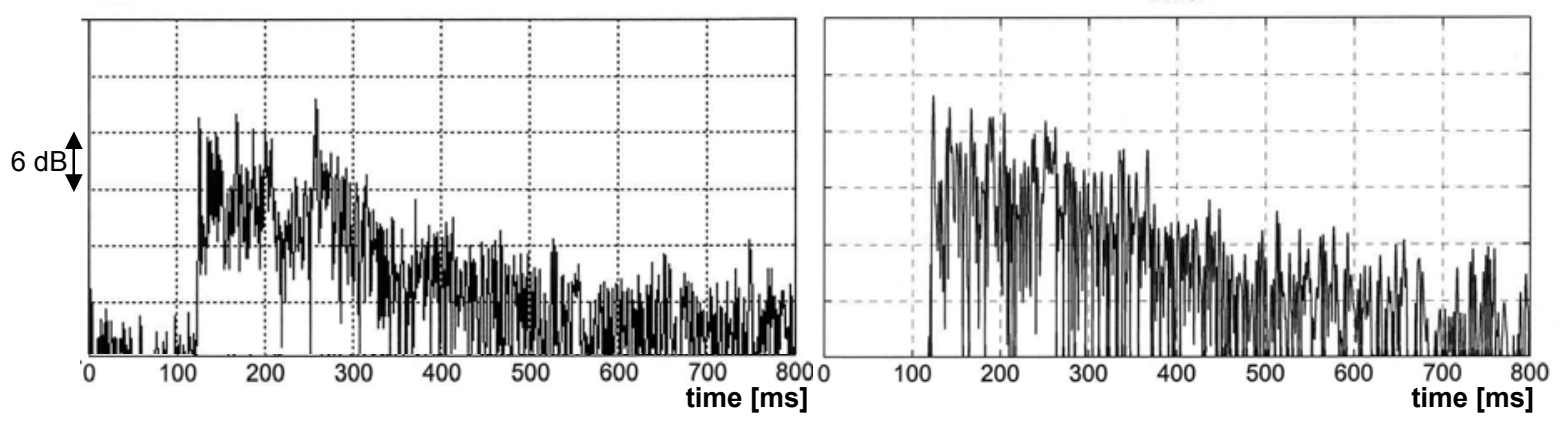

Figure 14.40. Measured ETC in the (real) Royal Albert Hall, measurement position M, with orchestra reflector and with mushrooms. Left: before renovation, Right: after renovation (after rearranging the mushrooms).

For this research it was found that the model gave slightly higher reflected sound levels than the real hall. This is because in reality, there will be more detail and therefore a little bit more diffusion than in the scale model. In that sense the scale model is "on the safe side"; when the problem is solved in the scale model, there is sufficient certainty that the problem will also be solved in reality.

The findings in the scale model, which showed that a sufficient reduction of the echo could be expected by rearranging the mushrooms, were confirmed in the real hall.

\subsubsection{Conclusions}

The Albert Hall used to be notorious for its echo. The ellipsoidal shape of the roof, actually a small part of the room, causes a geometrical amplification in a large range of the audience area. Further, the large dimensions of the hall cause a very low reverberant level for impulse excitation. As a result, the echo is well above reverberant level and is therefore audible.

In the situation without mushrooms and orchestra reflector, the sound pressure level in the Royal Albert Hall can be predicted by means of the geometrical method, since the source and receiver are well outside the focal point.

Outside the focal point we do see irregularities in the sound level, for which numerical methods can be used.

Geometrical and numerical methods can hardly be used for the evaluation of the more complex situation with mushrooms. For this purpose scale model research gives much more detailed insight. The alterations investigated in the scale model (rearranging the mushrooms) turned out to be effective in the real situation as well.

A few additional remarks can be made:

- The efficiency of the mushrooms depends on how well they shield the roof. At the rear of the stage, adjacent to the orchestra reflector there are some openings (see figure 14.39) that sometimes cause some audible echoes.

- The ground plan of the hall is elliptic. Although the reflection strength is reduced by the boxes and absorption of the audience, the strength of the early reflections can be attributed to this shape. The reflective areas adjacent to the organ can produce echoes in combination with highly placed sources (e.g., loudspeakers). 


\subsection{The "Klankkaatser"}

The "Klankkaatser" is a vertical prolate ellipsoid. The dimensions of the ellipsoid are: $a=b=2,5 \mathrm{~m}$ and $c=4 \mathrm{~m}$. The focal points are $3,12 \mathrm{~m}$ from the centre.

A part of the ellipsoid shape at the bottom, under the lower focal point, is missing. The pedestal gives access to the lower part. The conically shaped pedestal is sound absorbing. The wall material is polyester on a base of polystyrene. The inner side is made as smooth as possible to have low diffusion for high frequencies. The geometry is shown in figure 14.42. The geometrical accuracy is estimated to be within $1 \mathrm{~cm}$.

The "Klankkaatser" or "Sound-reverberator" is a piece of art made by the Dutch sound artist Hans van Koolwijk. The outer height is almost $10 \mathrm{~m}$.

In and around the focal point in the top of the Klankkaatser, a sound machine is positioned, which generates sound compositions. The lower part of the ellipsoid and pedestal is accessible. The visitor can hear the sound machine in the focal point. The Klankkaatser was opened in August 2010 in the "Muziekgebouw aan 't IJ" in Amsterdam [Koolwijk11].
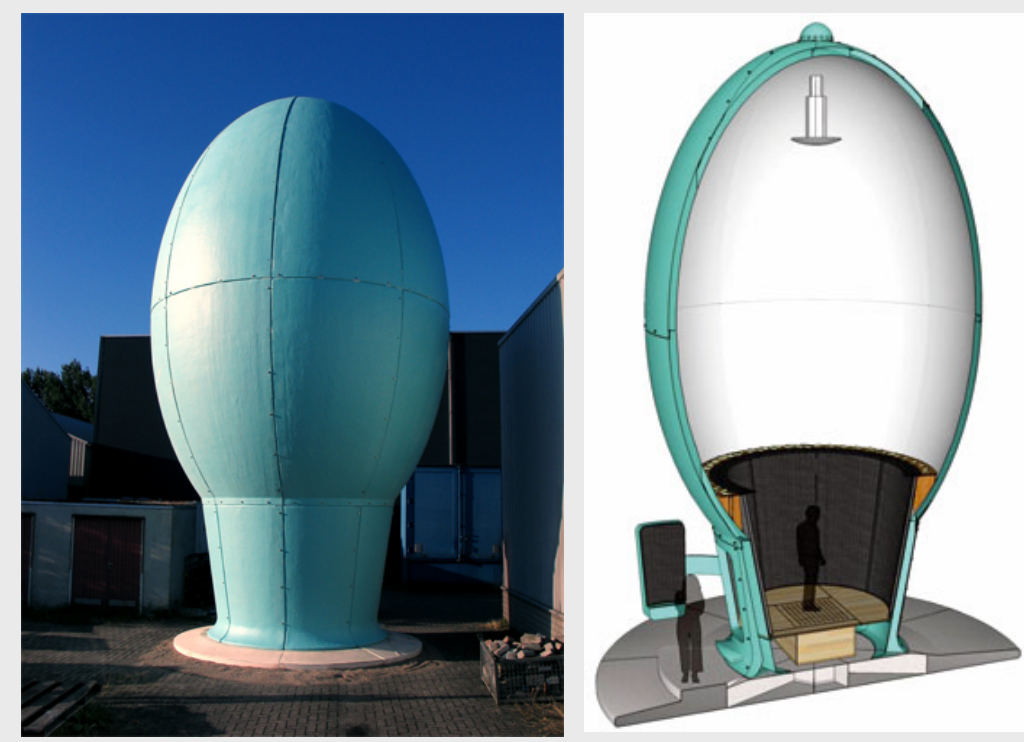

Figure 14.41. Picture of the outside of the Klankkaatser during construction (left) and $3 D$ visualization of a section of the Klankkaatser, right (drawing Farhan Alibux [Koolwijk11])

\subsubsection{Approximated sound level in the focal point.}

The source is assumed to be in the upper focal point. To estimate the sound pressure in the other focal point we have to consider the geometry, the a/c ratio and the missing part of the ellipsoid. The walls are assumed to be fully reflective. The pressure in the other focal point can be approximated from (9.4) and (7.3b):

$$
\left|p_{F}\right|=k \hat{p}\left(1-\cos \theta_{m}\right)\left(\frac{a}{c}\right)^{\sqrt{2}}
$$

In this specific case $\theta_{m}=0,5, a=2,5 \mathrm{~m}$ and $c=4 \mathrm{~m}:\left|p_{F}\right|=0,96 k \hat{p}$

The approximated sound pressure level is shown in figure 14.44. 


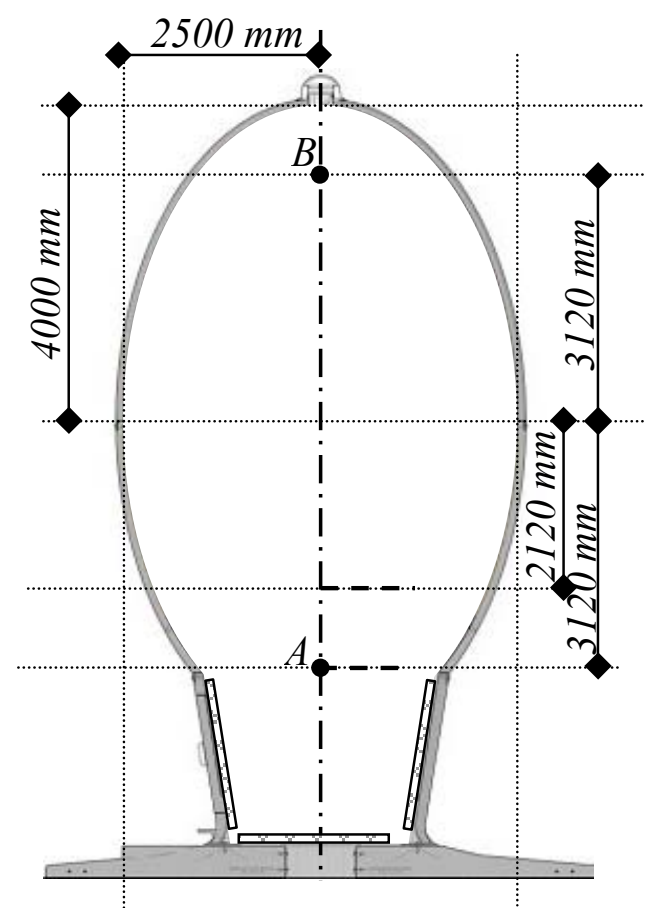

Figure 14.42. Cross section of the Klankkaatser with the main dimensions. B=upper focal point with sound source, A=lower focal point, microphone. Horizontal dashed lines: measurement lines.

\subsubsection{Numerical approximation}

The reflected sound pressure is calculated in the ellipsoid with a source in the upper focal point. The calculations are performed with (6.19), making use of (9.2). Figure 14.43 shows the calculated reflected sound field at 800 and $2 \mathrm{kHz}$.
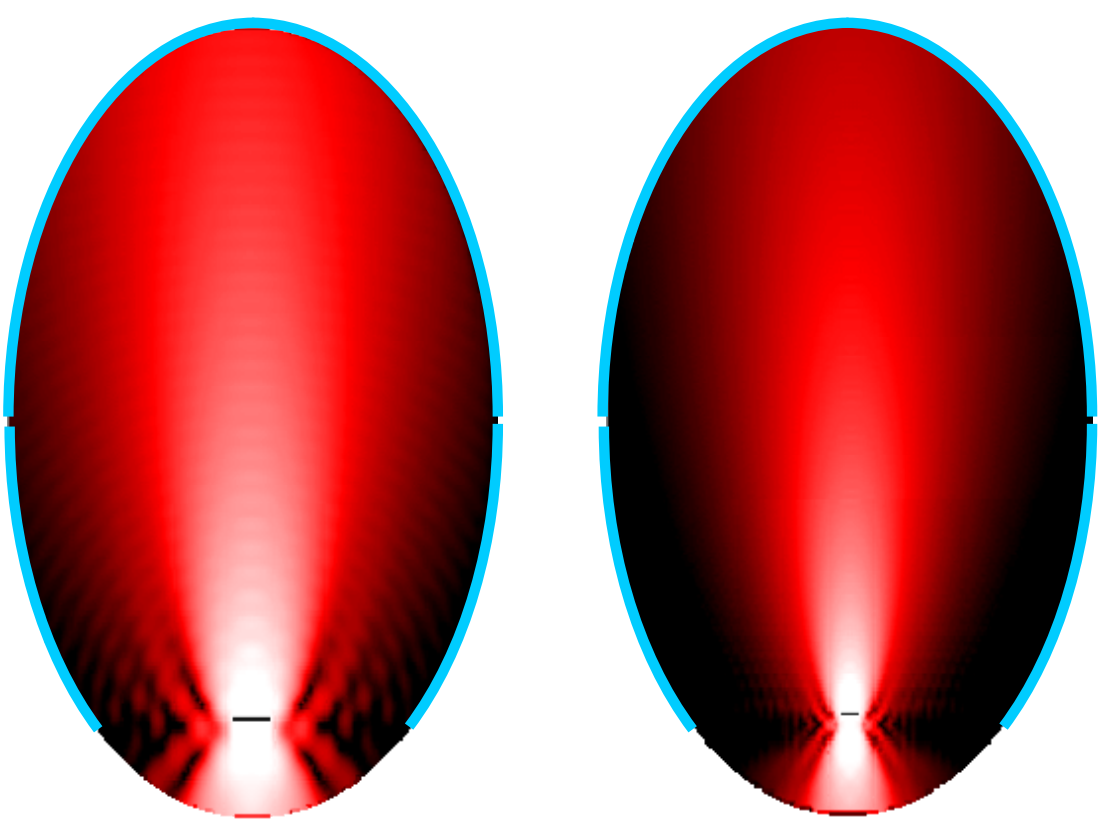

Figure 14.43. Numerically calculated reflected sound $\Delta L_{1 m}$ in the Klankaatser. Color range: $30 \mathrm{~dB}$ re SPL $1 \mathrm{~m}$ from source. Blue line: reflecting surface. Left: $800 \mathrm{~Hz}$; white: $\geq 15 \mathrm{~dB}$, black: $\leq-15 \mathrm{~dB}$; Right: $2000 \mathrm{~Hz}$; white: $\geq 20 \mathrm{~dB}$, black: $\leq-10 \mathrm{~dB}$ 
The graph shows that, outside the focal point, the energy is not equally distributed but concentrates around the $z$-axis.

The sound pressure in the focal point is given in figure 14.44 as a function of frequency. It shows that the approximation with (14.1) is close to the numerical results.

\subsubsection{Measurements}

Measurements were performed in the Klankkaatser with a source positioned at the upper focal point. The source consisted of a driver, a conical connection part and a 2 $\mathrm{m}$ long copper pipe with a diameter of $10 \mathrm{~mm}$. This will create an omni-directional sound source up to approx. $4 \mathrm{kHz}(\lambda=85 \mathrm{~mm})$, when the opening diameter is about $\lambda / 8$. The first internal reflection of the source will have a delay time of $11,6 \mathrm{~ms}$ (corresponding to $4 \mathrm{~m}$ ). In the lower focal point the path length difference between direct sound and first reflection will be $2\left(c-\sqrt{c^{2}-a^{2}}\right)=1,76 \mathrm{~m}$, corresponding to a delay time of $5,1 \mathrm{~ms}$.

The considerations considering the internal reflection of the source and the measurement technique can be found in chapter 11 .

A time window of $5 \mathrm{~ms}$ had been selected to be able to separate the direct sound from the reflection (see also figure 11.1).

The internal reflection of the source is kept outside the analysis time window.

The microphone was a $1 / 2$ inch $B \& K$ condenser microphone, positioned on a microphone pole. A fishing line was stretched horizontally across the Klankkaatser, at two different heights (see figure 14.42): at the height of the lower focal point and $1 \mathrm{~m}$ above, to enable accurate microphone positioning.

Because of the low level of the direct sound compared to the reflected sound (30-50 $\mathrm{dB}$ lower), the reference pressure at $1 \mathrm{~m}$ from the source was measured separately in the laboratory, as an average for different radiation angles.

The measured pressure in the lower focal point is presented in figure 14.44.

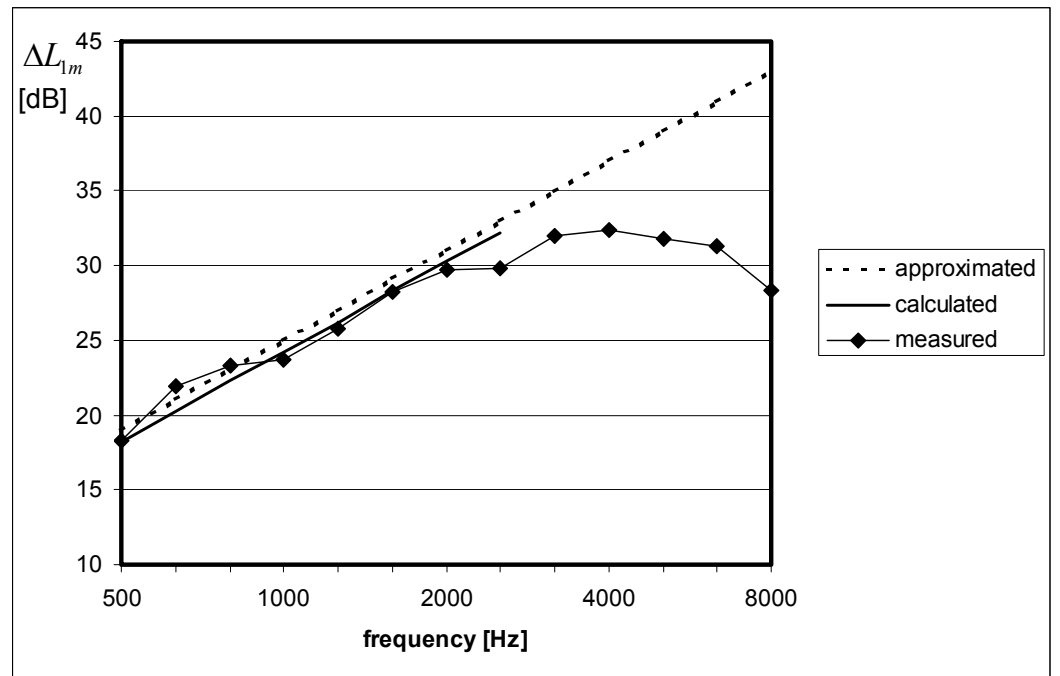

Figure 14.44. $\triangle L_{1 \mathrm{~m}}$, the $S P L$ in the lower focal point, relative to the SPL at $1 \mathrm{~m}$ from the source. Approximated SPL using (14.1), numerically calculated using (6.19) and measured.

For frequencies from $500 \mathrm{~Hz}$ to $2 \mathrm{kHz}$ the agreement with numerical results is satisfactory. At higher frequencies differences occur. This can be caused by: 
- inaccuracy of the geometry, which is expected to have a significant influence above $8 \mathrm{kHz}(1 \mathrm{~cm} \approx \lambda / 4)$.

- inaccuracy in the positioning of the source. The estimated possible error in source positioning is about $2 \mathrm{~cm}$. This may have a significant influence above $4 \mathrm{kHz}$.

- inaccuracy in the positioning of the microphone. The estimated possible error in source positioning is about $1 \mathrm{~cm}$. This may have a significant influence above $8 \mathrm{kHz}$.

The reason for small deviations in the range from 2500 to $4 \mathrm{kHz}$ is not known.

The pressure level is measured along the horizontal lines as indicated in figure 14.45. The distance between the measuring positions is $2 \mathrm{~cm}$. Also shown are the numerical results.
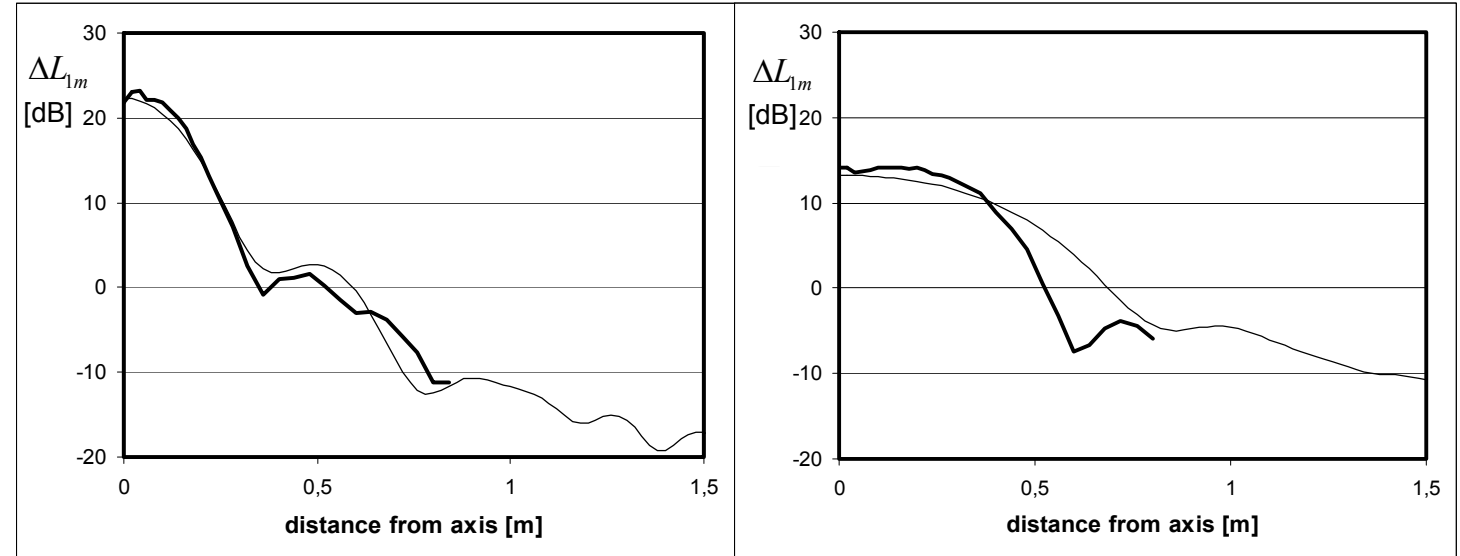

Figure 14.45. $\Delta L_{1 \mathrm{~m}}$, the $S P L$ relative to the $S P L$ at $1 \mathrm{~m}$ from the source, in the Klankkaatser along two horizontal lines (see figure 14.42): left: at $z=-3,12 m$ (through the focal point) and right: $z=-2,12 \mathrm{~m}$. Thin line: numerically calculated, thick line: measured. $800 \mathrm{~Hz}$ third octave band.

There is sufficient agreement. At longer distances from the focal point deviations will occur. To be able to separate the direct sound from the reflected sound in the measurement, the window is $5 \mathrm{~ms}$. Outside the focal point the reflected sound will arrive at different time delays. $1 \mathrm{~m}$ from the focal point the spread will be approximately $6 \mathrm{~ms}$; together with the duration of the source signal, this will mean that part of the energy will be outside the measurement window, causing the actual reflected pressure to be underestimated. 


\subsection{Office building with curved façade}

A new office building in the centre of Utrecht (Rabobank) has a curved façade. A 3D impression of the building is shown in figure 14.46. A plan of a typical floor is shown in figure 14.47.

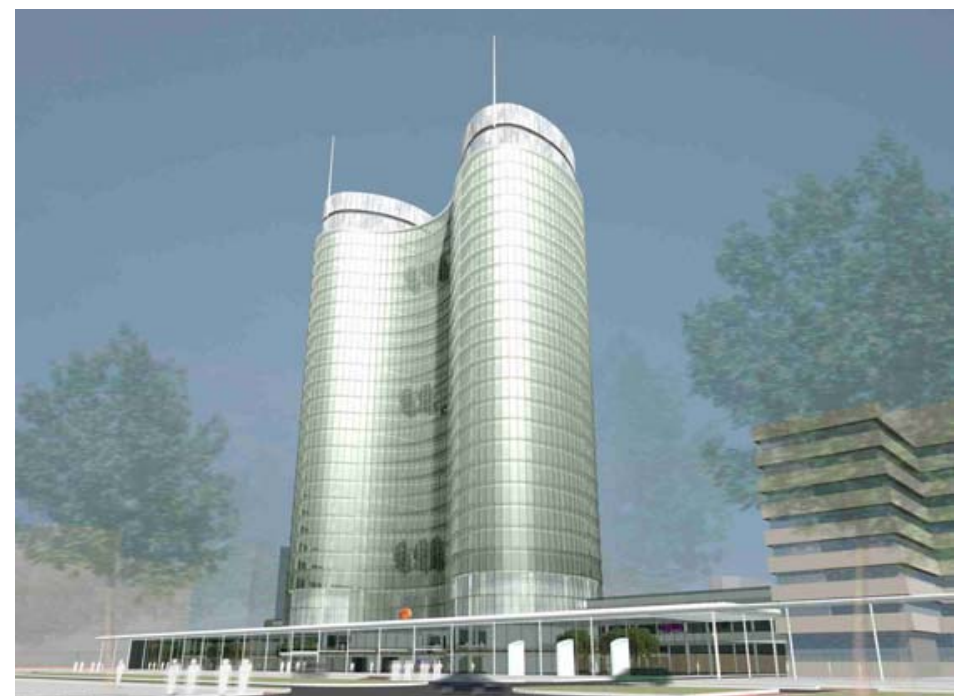

Figure 14.46. Impression of the new office building in Utrecht

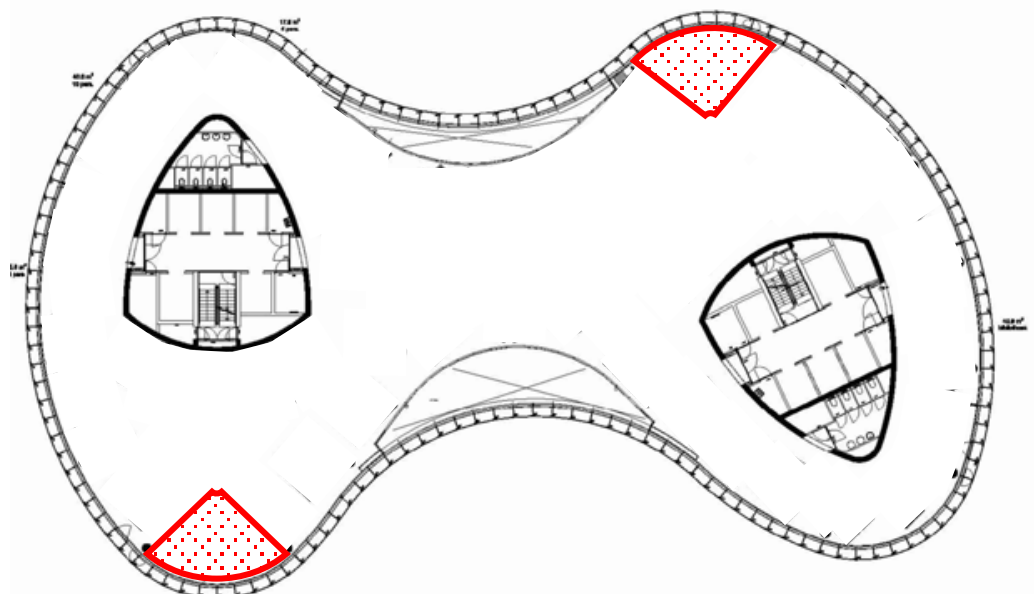

Figure 14.47. Typical floor plan with most critical areas indicated in red

The curved shape is made of planar glass panes with a width of $1,2 \mathrm{~m}$. Two areas are indicated where the façade (12 glass panes) forms a quarter cylinder with a radius of $7,7 \mathrm{~m}$. Especially in these areas amplification due to focusing is to be expected.

To predict the speech privacy, an estimation of the sound field was required.

The following methods were applied:

a. Calculation with ray tracing and image sources (ISM)

b. $\quad$ Calculation based on the wave field approximation, using formula (12.6)

c. Calculation based on the Kirchhoff integral (6.19)

d. Measurements

In all of these cases the source was positioned 1,5 $\mathrm{m}$ to the "left" of the center of the quarter cylinder. 


\section{$\underline{\text { 14.6.1 Ray tracing model }}$}

The sound pressure of the reflected sound is calculated with ray tracing, using CattAcoustics, version $8 \mathrm{i}$, area mapping. The SPL is shown for reflections between 0,001 and $100 \mathrm{~ms}$ after the direct sound. This excludes the direct sound. The results are shown in figure 14.48 .

Excluding contributions from the direct sound and (multiple) reflections from walls etc., a sound pressure level increase around the focusing point is observed. The calculated maximum sound pressure level is around $-14 \mathrm{~dB}$ relative to the SPL at 1 $m$ from the source $(100 \mathrm{~dB})$. The triangle shapes in the figure result from the overlap of reflected sound from multiple pane segments. Since all other surfaces are completely absorptive a focusing effect is visible, although the focusing area is rather wide. Including the contributions of other surfaces, the visibility of the focusing effect will be greatly reduced and can no longer be recognized; this can be seen in figure 14.49 .

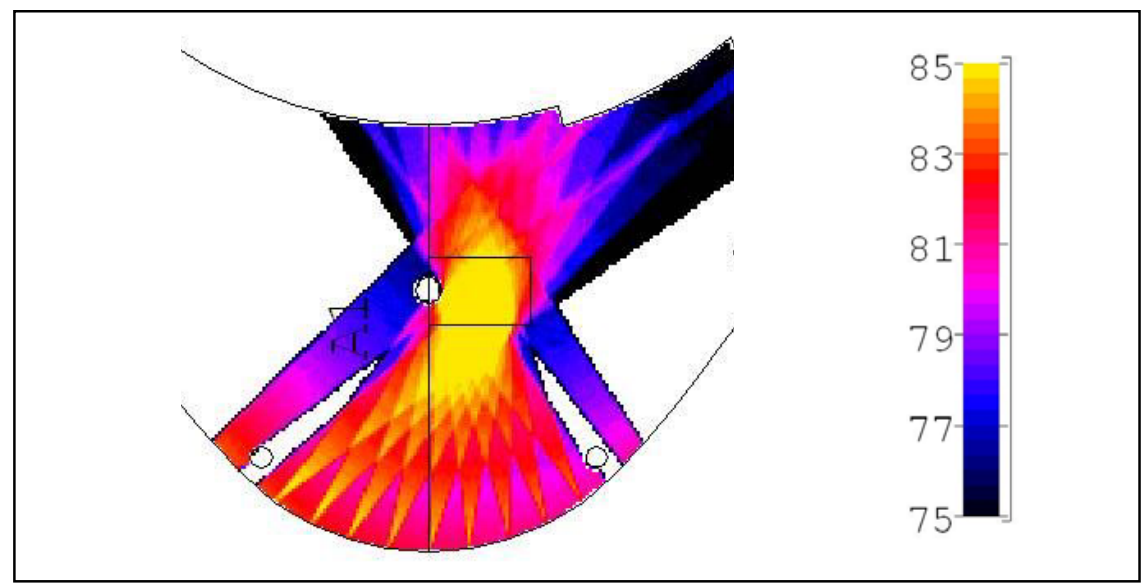

Figure 14.48. Reflected sound field calculated with ray tracing (Catt-Acoustics). The $1 / 4$ circle of the façade is reflecting; all other surfaces are 100\% absorptive. Source: $100 \mathrm{~dB}$ at $1 \mathrm{~m}$. Maximum pressure around focal point is $86 \mathrm{~dB}$.

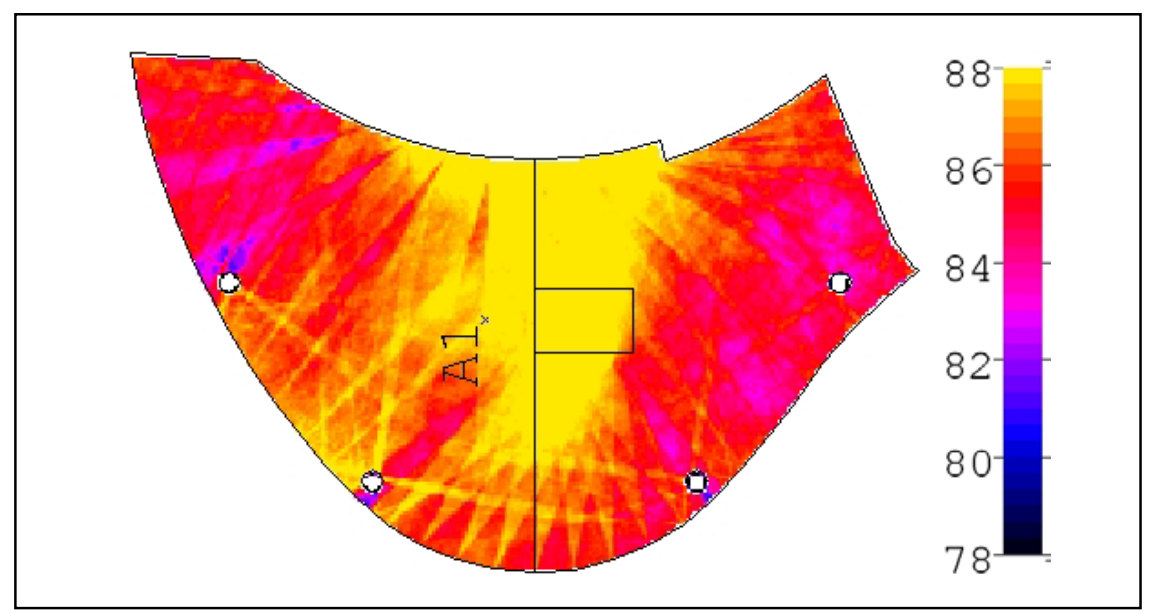

Figure 14.49. Reflected sound field calculated with ray tracing (Catt-Acoustics). The whole façade and other walls are reflecting; the floor is $100 \%$ absorptive, the ceiling $80 \%$. Source: $100 \mathrm{~dB}$ at $1 \mathrm{~m}$. 


\subsubsection{Image Source Method (ISM)}

With a radius of $7,7 \mathrm{~m}$ the average distance from the mirror sources to the receiver is $15,4 \mathrm{~m}$. With twelve panels contributing, the expected pressure will be approximately:

$$
\Delta L p_{1 m}=10 \log 12-20 \lg 15,4=-13 \mathrm{~dB} .
$$

The same computer model is also used for calculating the pressure in a line of points from the source (see figure 14.53) with ISM. The calculated pressure due to the reflected sound from the façade is about $-14 \mathrm{~dB}$ re $1 \mathrm{~m}$. The results are shown in figure 14.54 .

An alternative would be to add the contributions of all mirror sources, assuming equal phase. In that case the pressure would be approximately:

$$
\Delta L p_{1 m}=20 \lg 12 / 15,4=-2 \mathrm{~dB} .
$$

\subsubsection{Wave field approximation.}

The pressure in the focusing point of a cylindrical segment will be (12.6):

$$
\Delta L_{1 m}=20 \log \left(\frac{\theta_{m}}{\pi}\right)+10 \lg \left(\frac{\pi k}{R}\right)
$$

where $\theta_{m}=$ opening angle of the cylindrical segment, in this case: $\theta_{m}=0,28 \pi$

This expression assumes that the cylindrical segment is extended in the (vertical) $z$ axis to infinity. In reality the floor height is only $2,7 \mathrm{~m}$. For these dimensions the Fresnel integral is highly fluctuating, see figure 8.2. It is calculated that in this particular case (12.6) is about $2 \mathrm{~dB}$ too low.

The façade is segmented. For low frequencies it will act as a cylinder. For higher frequencies it will be more diffuse because it deviates from a perfect cylinder. This will be for frequencies in which a quarter wavelength is smaller than the 'depth' of the irregularity; in this case that will be $2,3 \mathrm{~cm}$ (see figure 14.50). This means that above $3,5 \mathrm{kHz}$ the focusing effect will be significantly less.

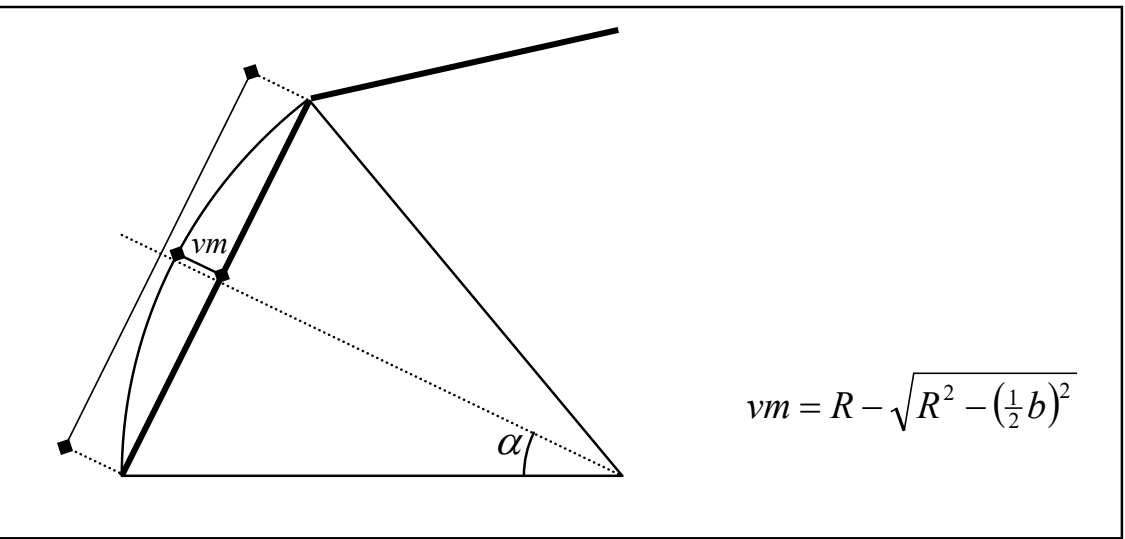

Figure 14.50. The depth $v m$ is the acoustical irregularity of a segmented circular shape.

For lower frequencies the segmenting should not influence the result of the wave field approximation. At $800 \mathrm{~Hz}$ the pressure increase relative to the position at $1 \mathrm{~m}$ will be, from (12.6): $-3,1 \mathrm{~dB}$. 
Since the source is not in the center but $1,5 \mathrm{~m}$ to the side, the pressure will be lower. According to (12.8) this reduction can be estimated: $-3,7 \mathrm{~dB}$, so the expected pressure will be $-6,8 \mathrm{~dB}$ (re $1 \mathrm{~m}$ ).

It should be noted that the position at $1,5 \mathrm{~m}$ is just outside the range of applicability, $x<0,75 \sqrt{\lambda R}$, so the estimate will too low. Limiting the reduction to $3 \mathrm{~dB}$ would result in a peak level of $-6 \mathrm{~dB}$.

\section{$\underline{\text { 14.6.4 Numerical approximation }}$}

The reflected sound field at $800 \mathrm{~Hz}$ (pure tone) is calculated for the 12 flat panels using (6.19) and shown in figure 11.12. The maximum reflected sound pressure is $2 \mathrm{~dB}$ relative to the sound level of the source at $1 \mathrm{~m}$. This is $4 \mathrm{~dB}$ higher than the analytical value, of which $2 \mathrm{~dB}$ can be attributed to the limited height. The numerical result corresponds to the addition of coherent mirror sources, (see par.14.6.2).
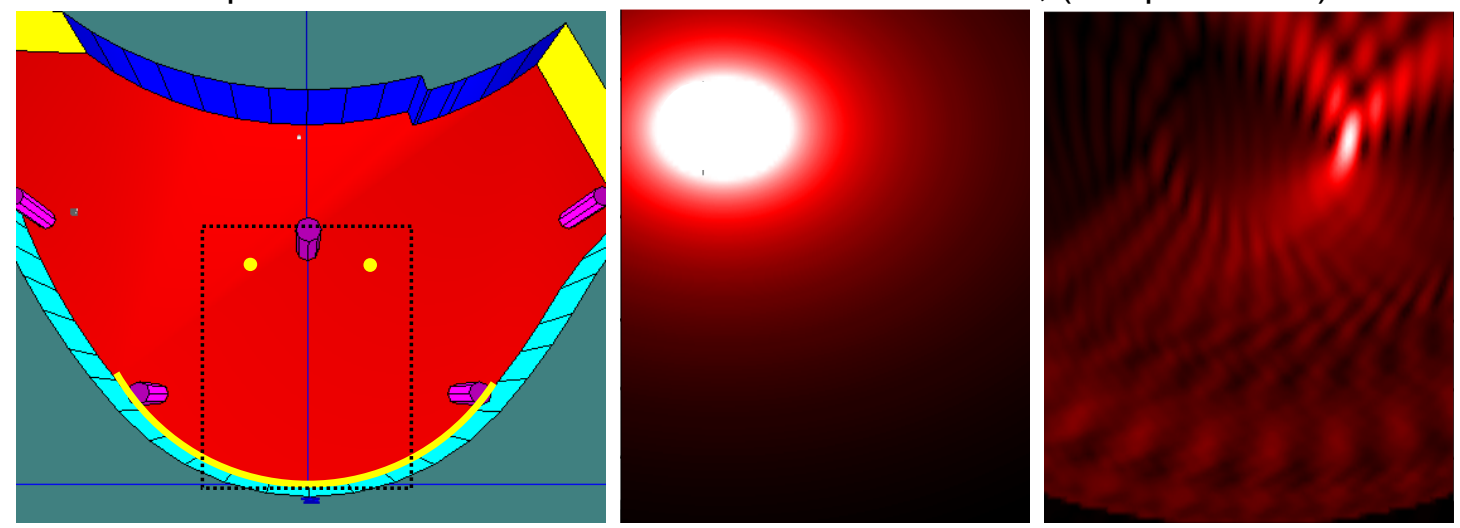

Figure 14.51. Calculation of the sound field. Left: indication of the source and focal point (yellow dots), the façade segments (yellow line) and the calculated area (dotted rectangle); middle: sound pressure (relative to $\hat{p}$ ) due to direct signal (white $\geq 1$ ); right: calculated reflected field using (6.19) at $800 \mathrm{~Hz}$.

\section{$\underline{\text { 14.6.5 Measurements }}$}

The measurements were made with a point source and MLS measurement system. A picture of the site during measurements is shown in figure 14.52. The ceiling is a good sound absorbing ceiling $\left(\alpha_{w}=0,85\right)$ and there is carpet on the floor.

Measurements are taken along a line through the source and the focusing point, as indicated in figure 14.53. The results are related to the sound pressure at $1 \mathrm{~m}$ from the source. The measurements are filtered for the $800 \mathrm{~Hz}$ third-octave band.

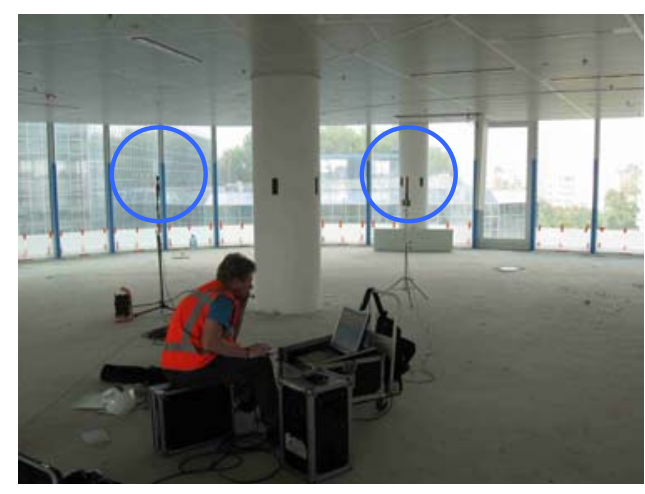

Figure 14.52. View towards the glass façade, right circle: point source, left circle: microphone

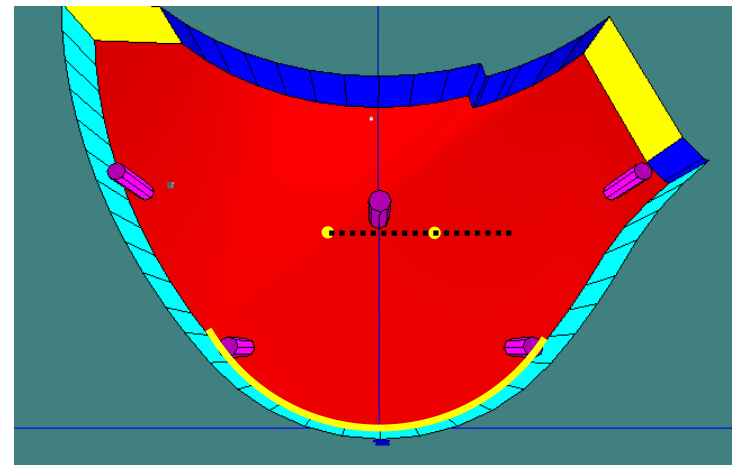

Figure 14.53. Line indicating the positions of the measurements 
The measurement results along the indicated line are shown in figure 14.54. Also shown is the calculated value using (6.19) for the third octave band of $800 \mathrm{~Hz}$ (resulting from a third octave band synthesis, as an average of 11 frequencies within this band). The maximum pressure measured is $-4,3 \mathrm{~dB}$ (re $1 \mathrm{~m}$ ), which is somewhere in between the analytical and numerical results shown above. Although there is not an exact match of the interference pattern, the order of magnitude of the measured focusing corresponds to the calculations with the wave based method (6.19). One possible reason for the differences is the presence of additional reflection surfaces (left of the left column and right of the right column in figure 14.53), which are no longer on the cylinder but will still contribute to the pressure field.

The calculated levels with ray tracing and ISM are $10 \mathrm{~dB}$ too low and extend over too large area. This is the result of the addition of energy instead of the addition of pressure with equal phase.

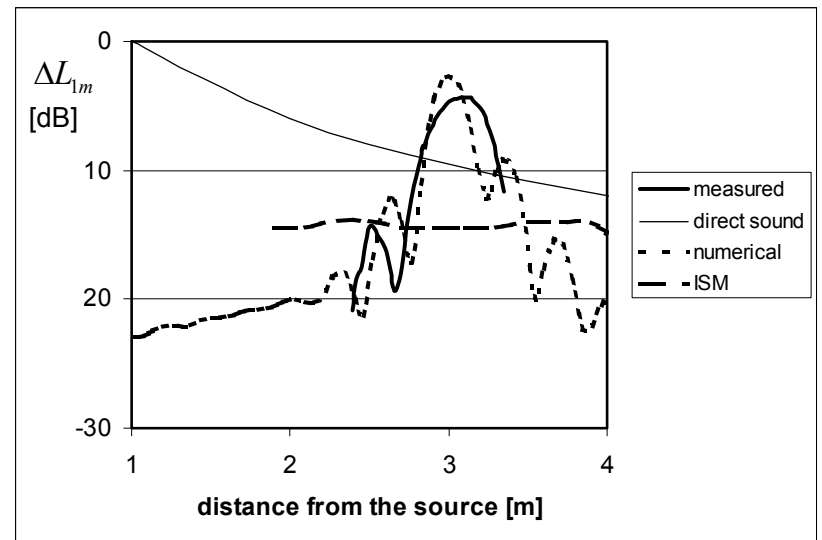

Figure 14.54. $\Delta L_{1 m}$, the $S P L$ relative to the $S P L$ at $1 \mathrm{~m}$ from the source, at $800 \mathrm{~Hz}$ along the line indicated in figure 14.53. Measured: with MLSSA. Numerical: calculated using (6.19). Direct: theoretical SPL as a reference. ISM: result ISM computer model. 


\subsection{Ellipsoidal meeting room}

\section{$\underline{14.7 .1 \text { Geometry }}$}

The meeting room of an office building in Assendelft has an ellipsoidal shape (see figure 14.55). The main deviations from a full ellipsoid are:

- a horizontal floor

- $\quad$ a door opening (about $2 \mathrm{~m}^{2}$, see figure 14.59)

- $\quad$ some small circular window openings (depth ca. $20 \mathrm{~cm}$ )
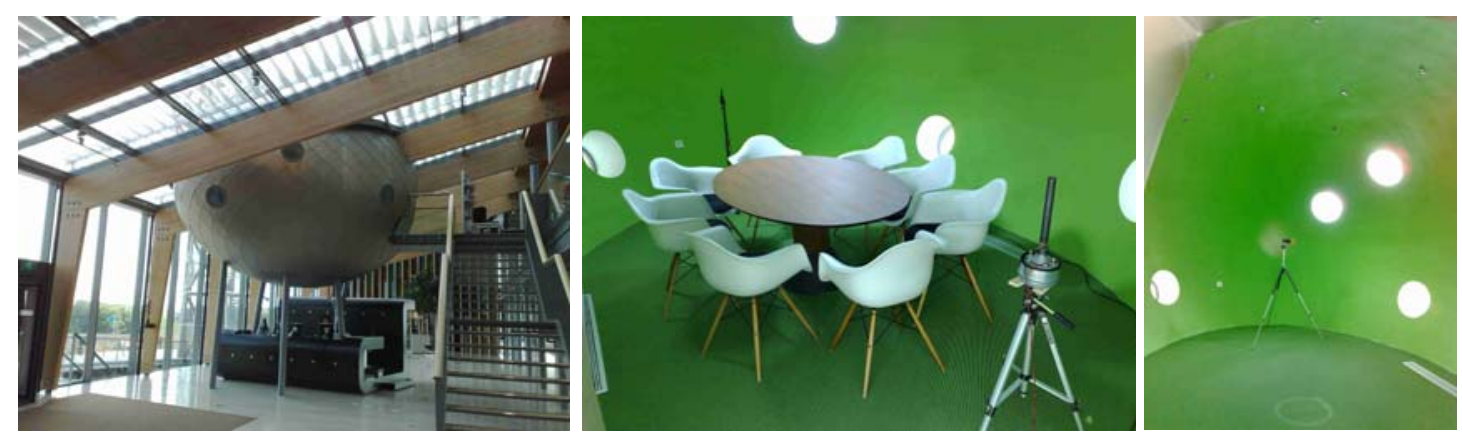

Figure 14.55. Ellipsoidal meeting room

The basic shape of the room is a prolate ellipse (see section 9) with $a=b=2,07 \mathrm{~m}$, $c=2,93 \mathrm{~m}$. The distance from the focal points to the center is $2,07 \mathrm{~m}$. A schematic section of the room is given in figure 14.56 .

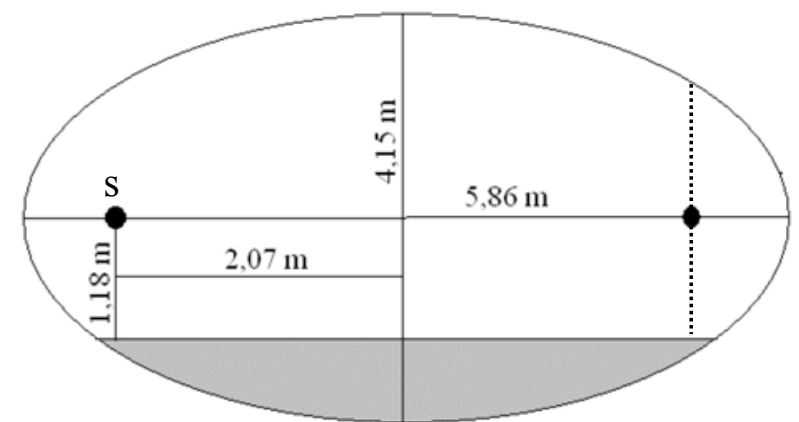

Figure 14.56. Dimensions of ellipsoidal meeting room, $s=$ source, dotted line: calculation and measurement positions.

The walls are plastered. The curvature of the room was made by hand, so there are some surface irregularities in the ellipsoid. From laser measurements the deviations are estimated to be around $+/-1 \mathrm{~cm}$.

The situation is evaluated using the following methods:

a. calculation based on the analytical approximation, formula (9.4)

b. calculation based on the Kirchhoff integral (6.19)

c. measurements

In all of these evaluations the source was positioned in one of the two focal points, $2,07 \mathrm{~m}$ from the center. 


\subsubsection{Wave field approximation.}

The pressure in the focal point of a full ellipsoid would be, from (12.3) and (12.10):

$$
\Delta L_{1 m}=20 \lg 2 k+28 \lg \left(\frac{a}{c}\right)
$$

Accounting for the reduction $r_{d}$ of the direct sound from the source to receiver position the pressure level increase relative to the direct sound $\Delta L$ will be:

$$
\Delta L_{1 m}=20 \lg 2 r_{d} k+28 \lg \left(\frac{a}{c}\right)
$$

For example, for $630 \mathrm{~Hz}$ the increase in this case would be $35,4 \mathrm{~dB}$. This is for a full ellipsoid. Due to the deviations mentioned, the SPL will be slightly lower.

\subsubsection{Numerical approximation}

The sound field is calculated using (6.19), as well as (9.2). In this integration the absence of the floor and the door opening is taken into account.

The pressure in the focal point, relative to the SPL of the direct sound is given as a function of frequency in figure 14.57. Due to the (missing) influence of the floor and the door opening, the numerical value is $3 \mathrm{~dB}$ lower than the analytical value.

The sound pressure at $630 \mathrm{~Hz}$ along a vertical line through the focal point is shown in figure 14.58 .

\subsubsection{Measurements}

The measurements were made with a point source and MLS measurement system. During the measurements there was no furniture present in the room.

The measured pressure in the focal point, relative to the SPL of the direct sound is given as a function of frequency in figure 14.57. The measurement results correspond to the numerical data, especially for the lower frequencies. For higher frequencies some deviations occur. This might be caused by small inaccuracies in the positioning of the source and the receiver or by inaccuracies of the shape of the ellipsoid.

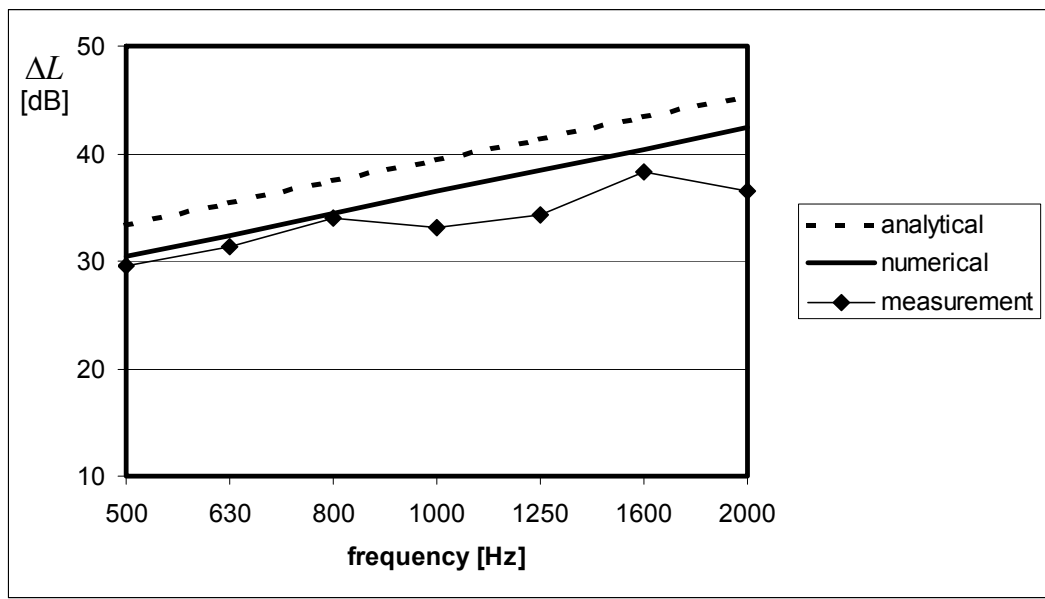

Figure 14.57. $\triangle L$, the SPL in the focal point relative to the direct sound, with the sound source in the other focal point. Results from wave field (analytical) calculation (14.3), numerical calculation (6.19) and the measurements. 
Measurements are also taken along a vertical line through the focal point, as indicated in figure 14.56. The measurements are filtered for the $630 \mathrm{~Hz}$ third-octave band.

The measurement results, expressed in $\Delta L$, are indicated in figure 14.58. These results show that the measurement result (thick solid line) is rather close to the numerical solution (thin solid line). The small remaining differences may be due to small inaccuracies of the source positioning, the deviations in the geometry and the influence of the reflection from the floor.

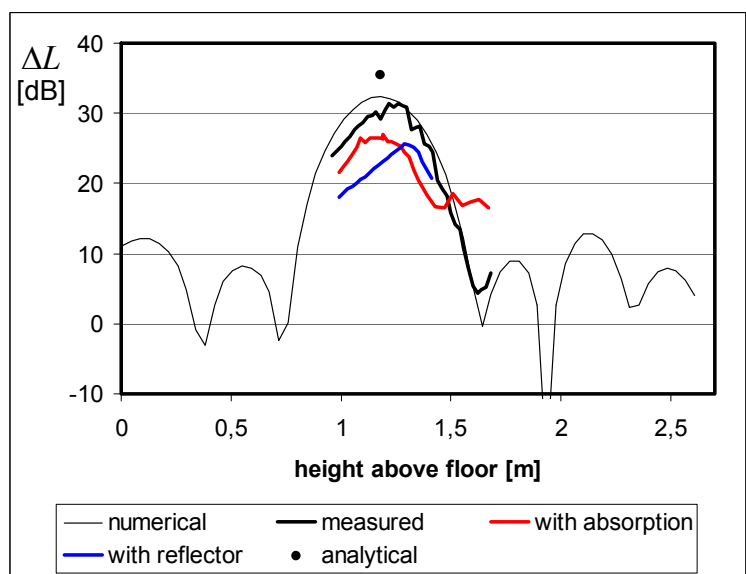

Figure 14.58. $\triangle L$, the SPL relative to the direct sound, in a vertical line through the focal point, $f=630 \mathrm{~Hz}$.

Due to the acoustical problems that occurred while using this room, some improvements had to be made. The walls were covered with absorbing material from approx. 0,8 to $2 \mathrm{~m}$ height (small circular elements filled with $7 \mathrm{~cm}$ of mineral wool). The ceiling part was covered with a convex reflecting panel, see figure 14.59. The results of the focusing with just the absorbing material and the combination of the absorbing material and the reflecting panels are shown in figure 14.58 for the $630 \mathrm{~Hz}$ third octave band. Both measures have an effect on the focusing. The total reduction is about $10 \mathrm{~dB}$. The remaining focusing will be caused by the lower part of the room. In normal use, with table and chairs, a further improvement can be expected.

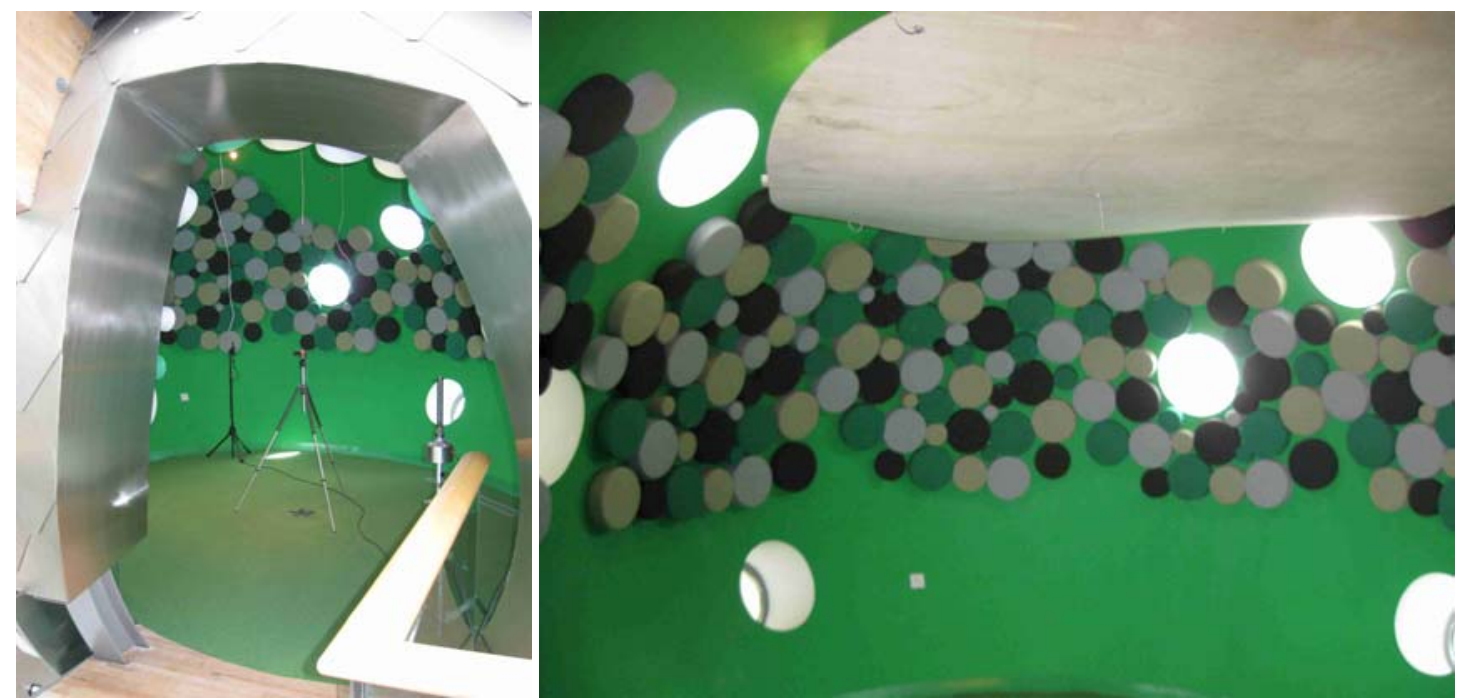

Figure 14.59. Ellipsoidal meeting room with acoustical improvements. On the walls absorbing material is positioned. Left: view from the outside to the inside (without reflector). Right: with convex reflector. 


\section{SECTION VI \\ CONCLUSIONS}

\section{Chapter 15 \\ Conclusions}

The results of this study are summarized in par. 15.1 .

Recommendations for further research are presented in par. 15.2.

\subsection{Results of the dissertation}

Many spaces, both in old and in modern architecture, have curved walls or ceilings. This research is devoted to the concentration of sound by concave curved surfaces. Although the phenomenon is well known and described in many standard works on acoustics, the quantification of this phenomenon was hardly known.

The concentrated reflection can cause an echo, depending on delay time and level relative to the direct sound. The concentrated reflection can also cause placedependent sound amplification and coloration by frequency-dependent amplification.

Outside the focusing point and for small wavelengths the sound field can be described to some extent using geometrical methods (chapter 5), when the interfering sound field is neglected or averaged out. Usually the curved surface is modeled with a number of flat segments. When using the image source method to approximate the correct value at the focusing point, using a small, frequencydependent size of the segments is required. It is not possible to calculate both the pressure at the focusing point and the width of the focusing area correctly, and the geometrical sound field may depend on the way the geometry is entered.

Using ray tracing, the pressure at the focusing area can be approximated to some extent when using a large number of segments (or exactly modelling the curved surface) in combination with a small, frequency-dependent receiver volume.

Beam Tracing, especially coherent BT, may be applicable outside the focusing area. Since it is a full geometrical method, an infinite SPL will be calculated at the focusing point.

In or around the focusing point, the pressure can be calculated or approximated using wave-based methods, which are described in chapters 6 through 10 .

In this work the wave theory is applied to room acoustical applications by extending the theory of piëzo-electric radiators used in acoustic microscopy to larger aperture angles and for sound sources outside the centre of a sphere.

Considering the reflection from a spherically curved surface (chapter 7 ), the pressure in the focusing point depends on the wavelength and the opening angle of the sphere segment. It does not depend on the radius of the sphere. The width of the peak pressure is also related to the wavelength (for a hemisphere the width is about half a wavelength). For small wavelengths the amplification is high but the area small, while for lower frequencies the amplification is lower, but the area is larger.

If it is the case that the wavelength is not small compared to the size of the reflector (if the 'depth' of the sphere segment is less than a wavelength) the sound is reflected more diffusely.

For sources outside the centre of the sphere segment, the maximum pressure in the focusing point will be less, due to imperfect focusing. 
The reflection from cylindrical surfaces (see chapter 8 ) is less pronounced than from spherically curved surfaces, since there is convergence in one direction and divergence in the other direction. Unlike spherically curved surfaces, the pressure in the focal point (or line) depends on the radius of the cylinder.

The reflection from ellipsoid surfaces (see chapter 9 ) is comparable to spherically curved surfaces with the difference being that there are two focal points. Thus, the source can be in one focal point and the receiver can be in the other, which makes it possible to have perfect focusing. The pressure in the focal point depends on the length/width ratio of the ellipsoid.

Additionally, an approximation method (see chapter 10) is presented for the reflection in the focusing area from a rectangular, double-curved surface.

A verification of use of the Kirchhoff integral for describing the reflection from a curved surface, is performed with a laboratory experiment (see chapter 11). The measurement results show good agreement with theory. Additional measurements are made in three slightly less controlled field situations (see par. 14.5, 14.6, and 14.7). Also in these cases a conformation of the conclusions from theory is found.

An engineering method for room acoustical applications is presented in chapter 12 . The cases presented in chapter 14 show that the order of magnitude of the sound concentration can be predicted using this engineering method.

For double-curved surfaces (ellipsoid or sphere), the focusing effect is quite strong for a small wavelength. Generally the possible reduction of the focusing effect by absorbers or diffusers is insufficient to eliminate the focusing effect. For cylindrical shapes, which have much lower pressure in the focusing line, these measures might be sufficient.

If diffusers are not able to sufficiently reduce the focusing effect, more drastic interventions are necessary such as changing the basic geometry of the room or adding large reflectors or redirecting panels. To obtain sufficient reduction of the specular reflection, the size of the redirecting panels and the rotation angle have to be sufficiently large.

Apart from the wave field approximation methods described in this work, an estimation of the problem and an evaluation of possible solutions can be obtained through acoustic scale model research. Two examples of halls with sound concentration problems, where scale model research was used to solve the problem, are described in chapter 14 (Tonhalle Düsseldorf and Royal Albert Hall London).

The results of this work may contribute (and in a number of cases already have contributed) to the understanding and quantification of the acoustical phenomenon of sound concentration from curved surfaces. This is relevant for analysing, solving or preventing acoustical problems that are related to focusing, thus contributing to the realisation of new architecture that is acoustically better fit for its purpose. 


\subsection{Recommendations for further research and developments}

From the results of this study the following recommendations can be given for further research and development.

- Echo perception: Although some methods are known, it is still not clear what the best echo-criterion should be. The methods studied by Yamamoto and Dietsch do give some indications, but the method studied by Dietsch and Kraak seems to underestimate the severity of the echo (par.14.3.4). Both methods can be used for measurements. For the design process better tools are needed. Additionally, a distinction should be made between which criteria should be used for echo detection or echo disturbance and which should be used for coloration.

- The use of the Kirchhoff integral for reflections only takes into account the first reflection. Although for most applications in room acoustics related to sound concentration this will be exactly what is needed, it would be worthwhile to be able to incorporate higher order reflections. This could be done by using higher order Borne approximations or by using BEM.

- Further research could explore time domain solutions if the full harmonic solution is available (e.g., by using BEM). It is noted, however, that for larger models, especially when covering the full frequency range, BEM is very time consuming.

- Geometrical computer models use plane reflecting surfaces. Both for ray tracing and for beam tracing an extension for curved surfaces is possible. This will not solve the basic limitations of those geometrical models in the focusing area, but it will improve the prediction outside the focusing area. It will also improve the capability of these models to indicate the occurrence and position of the focusing area.

- While the pressure in one focal point of an elliptical cylinder is known for the situation with the source in the other focal point, no expressions are available for the sound field with source and/or receiver outside the focal point.

- Although a numerical approximation could be derived for the pressure in one focal point of an ellipsoid for the situation with the source in the other focal point, no expressions are available for the sound field with source and/or receiver outside the focal point of an ellipsoid.

- Concerning double-curved surfaces: Although the error function can numerically be calculated, a further simplification of (10.13) would be helpful. At this moment a further simplification is not available. 


\section{References}

[Abramowitz70]

[AES01]

[Airy1871]

[Allen79]

[Bagenal31]

[Bagenal41]

[Barron71]

[Bate38]

[Bendat71]

[Beranek96]

[Berkhout82]

[Beyer99]

[Blancanus1620]

[Bolt50]

[Boone94]

[Born59]

[Chen93]

[Contant1860]

[Cox04]

[Cremer78]
Abramowitz,M.,Stegun,I, Handbook of mathematical functions, Dover Publ.Inc., New York, 1970.

Audio Engineering Society, Information document for room acoustics and sound reinforcement systems - Characterization and measurement of surface scattering uniformity, AES, 2001. Airy,G., On sound and atmospheric vibrations, with the mathematical elements of music, Macmillan, London,1868 $\left(1^{\text {st }}\right.$ ed.), 1871 ( $2^{\text {nd }}$ ed. $)$

Allen,J., Berkley,D., Image method for efficiently simulating small-room acoustics, J.Acoust.Soc.Am.65(4), 1979.

Bagenal,H., Wood,A., Planning for good acoustics, Methuen \& Co, London, 1931.

Bagenal,H., Concert music in the Albert Hall, Journal of the royal institute of Britisch architects, 1941.

Barron, M., The subjective effects of first reflections in concert halls - The need for lateral reflections, J. Sound. Vib. 15, 1971.

Bate,E., Note on the whispering gallery of St Paul's Cathedral, London, Proc. of the physical society 50, 1938.

Bendat,J.,Piersol,A., Engineering applications of correlation and spectral analysis, Wiley\& Sons Inc, New York, 1980.

Beranek,L., Concert halls and opera halls, how they sound, Acoust.Soc.Am, Woodbury, 1996.

Berkhout, A., Seismic migration, imaging of acoustic energy by wave field extrapolation, Elsevier, Amsterdam, 1982.

Beyer,R., Sound of our times, Two hundred years of Acoustics, Springer-Verlag, New York, 1999.

Blancanus,J.(latin: Biancani), Sphaera mundi, seu cosmographia demonstrativa (III. Echometria, idest Geometrica tractatio de Echo), 1620. http://libcoll.mpiwg-berlin.mpg.de/ libview?mode=imagepath\&url=/mpiwg/online/permanent/library/ GWS4WXH4/pageimg

Englisch translation: http://documenta.european-acoustics.net.

Bolt,R.,Doak,P., A tentative criterion for the short-term transient response of auditoriums, J. Acoust. Soc. Am., 22,1950.

Boone,M., Braat-Eggen,E., Room acoustics parameters in a physical scale model of the new music center in Eindhoven: Measurement method and results, Applied Acoustics 42, 1994.

Born,M., Wolf,E., Principles of optics, electromagnetic theory of propagation, interference and diffraction of light, Cambridge University Press, 1959, (seventh edition 1999).

Chen,X., Schwartz,K.,Parker,K., Radiation pattern of a focussed transducer: A numerically convergent solution, J.Acoust.Soc.Am.94(5)1993.

Contant,C., De Filippi,J., Parallele des principaux theatres modernes de l'Europe et des machines théatrales, 1860, reissued by Benjamin Blom, Inc.,New York, 1968.

Cox,T., D'Antonio,P., Acoustic absorbers and diffusers, theory, design and application, Spon Press, London, 2004.

Cremer,L., Müller,H., Die wissenschaflichen Grundlagen der Raumakustik (Band I \& II), S.Hirzel Verlag, Stuttgart, 1978. 
[Crunelle93]

[Dietsch86]

[Dillworth11]

[Dubout58]

[Dumont1766]

[Elmore85]

[Fasold94]

[Fuchs97]

[Geetere04]

[Gehler1787]

[Gertis94]

[Haas51]

[Hargreaves00]

[Hecht74]

[Hoffmann88]

[Huygens1678]

[ISO354]

[ISO3382-1]

[ISO3745]
Crunelle,M., Acoustic history revisited, The tuning of the world conference, Banff, Canada, 1993.

Dietsch,L.,Kraak,W., Ein objektives Kriterium zur Erfassung von Echostörungen bei Musik- und Sprachdarbietungen, Acustica 60,1986

Dillworth,C.,Smyth,S., The acoustic requirements of the Irisch Chamber Orchestra, Proc. IOA, Dublin, 2011.

Dubout,P., Perception of artificial echoes of medium delay, Acustica 8,1958.

Dumont,G., Parallele de plans des plus belles salles de spectacle de l'Italie et de la France, 1766

Elmore,W.,Heald,M., Physics of waves, Dover Publ., New York, 1985.

Fasold,W.,Schupp,G.,Stephenson,U., Modellmessungen und Rechnersimulationen als Entscheidungsgrundlage für raumakustische Verbesserungen am Plenarsaal des Deutschen Bundestages, IBP-Mitteiluing 251, 1994.

Fuchs, H.,Zha,X.: Acrylic-glass sound absorbers in the plenum of the Deutscher Bundestag. Applied Acoustics 51,1997.

de Geetere,L., Analysis and improvement of the experimental techniques to assess the acoustical reflection properties of boundary surfaces, Ph.D.-thesis, KU Leuven, 2004.

Gehler,J., Physikalische Wörterbuch, 1787 (Echo, Wiederhall). Gertis,K., Raumakustische Maßnahmen für den Plenarsaal, Bauzeitung 48, 1994.

Haas,H., Über den Einfluss eines Einfachechos auf die Hörsamkeit von Sprache, Acustica 1, 1951.

Hargreaves,T., Cox,T., Lam,Y., D’Antonio,P., Surface diffusionb coefficients for room acoustics : free-field measures, J.Acoust.Soc.Am.108(4),2000.

Hecht,E.,Zajac,A.:Optics, Addison-Wesley Pub.Co. , San Francisco, 1974.

Hoffmann,D., Vergleich von realen und simulierten akustischen Raum-Stoßantworten und deren Beurteilung, Diplomarbeit RWTH Aachen, 1988.

Huygens,C., Traité de la lumière, 1678, see also :

Thompson,S. (English transl.), Treatise on Light, http://www.gutenberg.org/ebooks/14725, 1912, and

Oeuvres complètes de Christiaan Huygens, Martinus Nijhoff, The Hague, 1888)

Acoustics - Measurement of sound absorption in a reverberation room, ISO 354, 1986.

Acoustics. Measurement of room acoustic paramaeters. Part 1 Performance spaces, ISO 3382-1, 2009.

Acoustics - Determination of sound power levels of noise sources using sound pressure - Precision methods for anechoic and hemi-anechoic rooms, ISO 3745, 2003. 
[ISO9613]

[ISO10534-1]

[ISO10534-2]

[ISO17497-1]

[Izenour77]

[Jardine03]

[Jean07]

[Kircher1650]

[Kircher1671]

[Kirkup07]

[Koolwijk11]

[Kuttruff73]

[Kuttruff93]

[Langhans1810]

[Lautenbach10]

[Lochner58]

[Long06]

[Lorenz01]

[Lorenz06]

[Lucas82]

[Makrinenko86]

[McNamara90]

[Mechel09]
Acoustics- Attenuation of sound during propagation outdoors- Part I: Calculation of the absorption of sound by the atmosphere ISO 9613, 1993.

Acoustics - Determination of sound absorption coefficient and impedance in impedance tubes - Part 1: Method using standing wave ratio, 1996.

Acoustics - Determination of sound absorption coefficient and impedance in impedance tubes - Part 2: Transfer-function method, 1996.

ISO, Acoustics - Sound-scattering properties of surfaces - Part 1: Measurement of the random-incidence scattering coefficient in a reverberation room, ISO 17497-1, 2004.

Izenour,G., Theater design, Mc Graw Hill, 1977.

Jardine,L., On a grander scale: The outstanding life of sir Christopher Wren, HarperCollins, New York, 2003.

Jean,P., Noe,N., Gaudaire,F., Calculation of tyre noise radiation with a mixed approach, Acta acustica - Acustica, 2007.

Kircher,A., Musurgia universalis, 1650.

Kircher,A.: Ars magna lucis et umbrae, 1671.

Kirkup,S. The Boundary Element Method in acoustics, 2007, www.boundary-element-method.com/tbemia07.pdf

www.klankkaatser.nl

Kuttruff,H.,Room acoustics, Elsevier Science Publ.,1973, (fourth edition, Taylor \& Francis, London, 1999)

Kuttruff,H.: Some remarks on the simulation of sound reflection from curved walls. Acustica 77, 1993.

Langhans,C., Über Theater oder Bemerkungen über Katakustik in Beziehung auf Theater, 1810.

Lautenbach,M., Vercammen,M., Volume diffusers in the reverberation room, Proc. ICA, Sydney, 2010.

Lochner,J., Burger,J., The subjective masking of short time delayed echoes by their primary sounds and their contribution to the intelligibility of speech, Acustica 8, 1958.

Long,M., Architectural acoustics, Elsevier Academic Press, Burlington, 2006.

Lorenz, K.-H., Auralisation of a scale model of the Royal Albert Hall, NAG Journaal nr 158, 2001.

Lorenz-Kierakiewitz, K.-H., Vercammen,M., From knocking ghost ot excellent acoustics - The new Tonhalle Düsseldorf: innovative design of a concert refurbishment, Proc. IOA, Kopenhagen, 2006.

Lucas,B., Muir,T., The field of a focussing source, J.Acoust.Soc.Am.72(4), 1982.

Makrinenko,L., Acoustics of auditoriums in public buildings, Acoust.Soc.Am., Woodbury, 1994 (Translation of Akustika pomeshchenii obshchestvennykh zdanii, 1986)

McNamara, D., Pistorius,C., Malherbe,J., Introduction to the uniform geometrical theory of diffraction, Artech House, 1990. Mechel,F., Raumakustische Felder, S. Hirzel Verlag, Stuttgart, 2009. 
[Metkemeijer02]

[Meyer52]

[Mommertz95]

[Mommertz96]

[Moreno81]

[Muncey53]

[Nat.Phys28]

[Niese61]

[O'Neil49]

[Okano01]

[Patte1782]

[Petzold 27]

[Raman21]

[Rayleigh1896]

[Rife89]

[Rindel85]

[Ripoll01]

[Rollins05]

[Sabine22]

[Sumbatyan07]
Metkemeijer,R., The acoustics of the auditorium of the Royal Albert Hall before and after redevelopment, Proc. IOA, London, 2002.

Meyer,E., Schodder,R., Über den Einfluss von

Schallrückwürfen auf Richtungslokalisation und Lautstärke bei Sprache, Math.-Phys. KI. 6, 1952.

Mommertz,E., Müller,K., Simulation der Schallübertragung in Räumen mit gekrümmte wandflächen, Daga, Saarbrücken, 1995.

Mommertz,E., Untersuchung akustischer Wandeigenschaften und Modellierung der Schallrückwürfe in der binauralen Raumsimulation, Ph.D. thesis RWTH Aachen, 1996.

Moreno,A., Zaragoza,J., Alcantarilla,F., Generation and suppression of flutter echoes in spherical domes, J.Acoust.Soc.Jpn.(E), 1981.

Muncey,R., Nickson,A, Dubout,P., The acceptability of speech and Music with a single artificial echo, Acustica 3, 1953.

Report on the Acoustics of the Royal Albert Hall of Arts and Sciences at Kensington, National Physical Laboratory, Physics department, London, 1928.

Niese, H., Die Messung der Nutzschall- und

Echogradverteilung zur Beurteilung der Hörsamkeit in Räumen, Acustica 11, 1961.

O'Neil,H., Theory of focussing radiators, J.Acoust.Soc.Am. 21, 1949.

Okano,T., Overhead localization in a hall with oval plan, and its practical solution, Proc. Acoust.Soc.Am, Fort Lauderdale,2001.

Patte,P., Eassai sur l'architecture theatrale, 1782 (reprint Minkoff, Genève, 1974).

Petzold, E., Elementare Raumakustik, Bauwelt-Verlag, Berlin, 1927.

Raman,C., Whispering-gallery phenomena at St. Paul's Cathedral. Nature 108:42, 1921.

Strutt,J., Baron Rayleigh, Theory of Sound, 1896, Dover Publications, New York, 1945.

Rife,D., Vanderkooy,J., Transfer-function measurement with maximum-length sequences, J.Audio Eng.Soc., 1989.

Rindel,J., Attenuation of sound reflections from curved surfaces", Proc. 24 ${ }^{\text {th }}$ Conf. on Acoustics, Strbské Pleso, 1985. Ripoll,J., Ntziachristos, V., Carminati,R., Nieto-Vesperinas, M., Kirchhoff approximation for diffusive waves, Physical review, 2001.

Rollins,S., The Salt Lake City Tabernacle: Acoustic characterization and study of spatial variation, Thesis Brigham Young University, 2005.

Sabine,W., Collected papers on acoustics, 1922, reprint by Dover publ. Inc., New York, 1964.

Sumbatyan,M., Boyev,N., Non-plane reflecting surfaces in room acoustics, Proc. ISRA, Sevilla, 2007. 
[Saunders1790]

[Shearer70]

[Stephenson94]

[Stephenson08]

[Tennhardt05]

[Trévoux1743]

[Trochin08]

[Utami05]

[Vercammen06a]

[Vercammen06b]

[Vercammen08]

[Vitruvius]

[Vorländer95]

[Vorländer08]

[Wahlström85]

[Williams99]

[Wulfrank06]

[WulfftenPalthe76]

[Wrobel02]

[Xiangyang03]
Saunders,G., A treatise on theatres, London, 1790, reissued by Benjamin Blom, Inc.,New York, 1968.

Shearer,K., The acoustics of the Royal Albert Hall, British Kinematography sound and television, 1970.

Stephenson, U., Zur Raumakustik großer kreisförmiger Räume am Beispiel des Plenarsaals des Deutschen Bundestages; Deutsche Bauzeitschrift, 1994.

Stephenson, U., Raumakustische Computersimulation, Vorlesung Un. Hamburg, 2008.

Tennhardt, H.-P., Behr,T., Winkler,H., Der Kuppelsaal - eine Aufgabe für die raumakustische Modellmesstechnik, Proc. DAGA, München, 2005.

Trévoux , Dictionnaire universel françois et latin ("Dictionnaire de Trévoux"),1743.

Trochin,L., Durvilli,I., Tarabusi,V., The marvellous sound world in the "Phonurgia Nova" of Athanasius Kircher, Proc.

Acoustics08, Paris, 2008.

Utami,S., An acoustical analysis of domes coupled to rooms, with special application to the Darussholah Mosque in East Jave, Indonesia, Thesis Brigham Young University, 2005.

Vercammen,M., Lorenz-Kierakiewitz, K.-H., Mercier,S., Vom Klopfgeist zu einer hervorragenden Akustik (1)- Die neue Tonhalle Düsseldorf, Proc. DAGA, Braunschweig, 2006. Vercammen,M., Metkemeijer,R., e.o.,Acoustics by Peutz, theatres and concert halls, Peutz bv, Mook, 2006.

Vercammen,M., The reflected sound field by curved surfaces, Proc. Acoustics08, Paris, 2008.

Vitruvius, De architectura libri decem, 24 B.C. book V, chapter V. (translation to Dutch: Handboek Bouwkunde, AtheneumPolak \& van Gennip, Amsterdam, 1997)

Vorländer,M., Revised relation between the sound power and average sound pressure level in rooms and consequences for acoustic measurements, Acustica 81, 1995.

Vorländer,M., Auralization, Springer-Verlag Berlin, 2008.

Wahlström,S., The parabolic reflector as an acoustical amplifier, J.Audio Eng. Soc.,1985.

Williams,E., Fourier acoustics sound radiation and nearfield acoustical holography, Academic Press, London, 1999.

Wulfrank,T., Orlowski,R., Acoustic analysis of Wigmore hall, London, in the context of the 2004 refurbishment, Proc. IOA, Kopenhagen, 2006.

van Wulfften Palthe,D., de Vries,D., Inleiding in de Akoestiek (c36), TH Delft, 1976.

Wrobel, L., The Boundary Element Method, Volume 1.

Applications in thermo-fluids and acoustics, J.Wiley \& Sons, Chichester, 2002.

Xiangyang,Z., Kean,C., Jincai,S.. On the accuracy of the raytracing algorithms based on various sound receiver models. Appl. Acoust. 64, 2003. 
Xu,A., Gallaud,N., Acoustic $1 / 10^{\text {th }}$ scale model test of concert hall in Tawan, Proc. IOA, Dublin, 2011.

[Yamada05] Yamada,Y., Hidaka,T., Reflection of a spherical wave by acoustically hard, concave cylindrical walls based on the tangential plane approximation, J.Acoust.Soc.Am.,2005.

[Yamamoto71] Yamamoto, T., The perceptible limit of the echo due to multiplex reflections, J. Acoust. Soc. Jap. ,1971.

[Yokota04] Yokota,T., Sakamoto,S., Tachibana,H., Ikeda,M., Takahashi,K., Ooturu,T., Comparison of room impulse response calculated by the simulation methods based on geometrical acoustics and wave acoustics, Proc. ICA, Kyoto, 2004. 


\section{Appendices}

\section{Appendix 1}

\section{List of symbols}

$\alpha \quad$ angle between source and point on $S \quad$ [rad]

$\delta(r) \quad$ spatial delta pulse $\quad[-]$

$\delta \quad$ drop rate of a masking curve $\quad[\mathrm{dB} / \mathrm{s}]$

$\Delta t \quad$ time delay, travel time [s]

$\Delta L \quad S P L$ of the reflection relative to the direct sound $\quad[\mathrm{dB}]$

$\Delta L_{1 m} \quad S P L$ of the reflection relative to the direct sound $1 \mathrm{~m}$ from the source [dB]

$\Delta L_{r} \quad$ SPL of the reflection, only due to difference in traveled distance relative to the direct sound [dB]

$\Delta L_{c} \quad S P L$ of the reflection of a concave reflector relative to a flat reflector [dB]

$\Delta L_{f} \quad$ difference in SPL of the reflection in a specific point compared to the SPL in a reference point (focusing point, focusing point with source in the centre, focusing point in a sphere) [dB]

$\varepsilon \quad$ error in the phase

$\phi \quad$ angle with the $\mathrm{x}$-axis in the $\mathrm{x}$-y plane

$\varphi \quad$ angle between line from receiver to point on $S$ and the normal on $S \quad$ [rad]

$\eta \quad$ relative error

$[-]$

$\lambda \quad$ wavelength

$\theta \quad$ angle of the incident sound, angle with the z-axis

[m]

$\theta_{m} \quad$ opening angle of the segment [rad]

$\theta_{r} \quad$ angle of the receiver to the axis of the sphere segment

$\theta_{s} \quad$ angle of the source to the axis of the sphere segment

$\theta_{v} \quad$ maximum visibility angle for reflections against a plane surface

$\rho \quad$ density of air

$\left[\mathrm{kg} / \mathrm{m}^{3}\right]$

$\omega \quad$ angular frequency of the signal

$\Delta \Omega \quad$ opening angle of the beam $[\mathrm{rad} / \mathrm{s}]$

[rad]

a radius of a radiating disk; dimension of an ellips or ellipsoid [m]

A receiver

$b \quad$ characteristic dimension (width) of plane surfaces; dimension of an ellipse or ellipsoid

$[-]$

$B$ source

$c$ speed of sound dimension of an ellipsoid

$C_{1}, C_{3}$ phase parameters (chapter 10)

$C_{2}, C_{4}$ phase parameters (chapter 10)

$d \quad$ traveled distance of the reflected path

$d_{\text {geo }}$ distance form the source where the geometrical reflection will change into spherical propagation

[m]

$[-]$

$d S \quad$ surface element

$D \quad$ diameter of receiver volume

$E$ energy

$[-]$ 
EI elliptic integral

$E K \quad$ echo indicator ("echokriterium") according to [Dietsch86]

$[-]$

$f$ focal point: focussing point for plane incident waves, $f=R / 2$

$f$ frequency

$f(A) \quad$ pressure in $A$ related to the pressure in the focusing point

$[-]$

$G(r, \omega)$ Green's function

$[-]$

I sound intensity

$j \quad$ complex number $j=\sqrt{-1}$

$J_{n}(x) \quad$ Bessel function of $\mathrm{n}$-order

$k$ acoustic wavenumber

$l_{i} \quad$ path length of ray $I$

$L_{p} \quad$ sound pressure level (SPL)

$L_{p 1 m} \quad S P L$ at $1 \mathrm{~m}$ from the source

$M \quad$ Mirror source

$[-]$

$\bar{n} \quad$ normal vector on surface $S$

$[-]$

$N \quad$ number of reflecting surfaces

$[-]$

$O \quad$ origin $(0,0,0)$

$[-]$

$n(z) \quad$ number of contributing surfaces depending on the position on the $z$-axis.

$\hat{p} \quad$ amplitude descriptor, representing the pressure at $1 \mathrm{~m}$ from a source $[\mathrm{N} / \mathrm{m}]$

$p(x)$ sound pressure (function of distance $x$ )

$[\mathrm{Pa}]$

$p_{r m s}^{2} \quad$ mean square value of the sound pressure

$\left[\mathrm{Pa}^{2}\right]$

$P \quad$ sound power

$[\mathrm{W}]$

$P(r, \omega)$ Fourier transform of the time signal $p(r, t)$

$[\mathrm{Pa}]$

$q \quad$ amplification of the reflected pressure from the concave surface relative to a flat surface

$[-]$

$r \quad$ distance from a source, focal point or mirror source coordinate in polair or cylindrical coordinate system

[m]

$r_{d} \quad$ distance between source and receiver

$[\mathrm{m}]$,

$R \quad$ radius

$[\mathrm{m}]$

$R^{2} \quad$ coefficient of determination of a regression line

$[-]$

$R_{Z} \quad$ complex reflection factor related to the surface impedance

$[-]$

$s \quad$ distance from the source to the surface element $d S$ on the reflector

$S \quad$ surface

$[\mathrm{m}]$

$\left[\mathrm{m}^{2}\right]$

$S(r) \quad$ cross-sectional area of the beam at distance $r$ from the source $\left[\mathrm{m}^{2}\right]$

$S_{u} \quad$ area of the undisturbed spherical wave front

$\left[\mathrm{m}^{2}\right]$

$S_{r} \quad$ area of the reflected geometrical wave front $\left[\mathrm{m}^{2}\right]$

$S P L \quad$ sound pressure level

$t \quad$ time

[s]

$t_{s} \quad$ center time

$[\mathrm{s}]$

$u \quad$ distance of the radiating element $d S$ to the receiver

[m]

$U \quad$ volume velocity of a monopole source

$\left[\mathrm{m}^{3} / \mathrm{s}\right]$

$[\mathrm{m}]$

$v(r, \omega)$ velocity

$v_{n}(r, \omega)$ velocity in the direction of $\bar{n}$, normal to the surface

$V \quad$ volume

$\left[\mathrm{m}^{3}\right]$

$w \quad$ distance of the reflector to the focal point

[m] width of the focusing area

[m]

$z_{f} \quad$ length of the focusing area 


\title{
Appendix 2.
}

\section{Curriculum Vitae}

\author{
Name: $\quad$ : Martijn L.S. Vercammen \\ Address : Stieltjesstraat 183, 6511 AK, Nijmegen. Netherlands \\ Date of birth. : Waalwijk, 02-10-1961 \\ Telephone. : : +31243570707; mobile: +31 650617432 \\ E-mail : M.Vercammen@mook.peutz.nl \\ Education: \\ 1974-1980 \\ 1980-1986 : Eindhoven University of Technology, Department of Architecture, \\ Building and Planning, unit Physics of the Built Environment \\ Internship J.Pierce Foundation, Yale University, New Haven, USA \\ 1986 \\ : End work Laboratory for Acoustics, on sound intensity \\ work: \\ 1986-now : Peutz bv, Mook, Netherlands \\ senior consultant from 1994, member of the board from 1998 \\ reponsibilities : - Reseach projects in the fields of room acoustics, building \\ acoustics and vibrations. \\ - Peutz Laboratory for Acoustics, Accredited according to \\ ISO 17025 \\ - Consulting activities in the field of room acoustics, building \\ acoustics and building physics for many building projects.
}

work related activities:

1991-now : teacher at PAO course on environmental and building acoustics

1995-now : member of the Dutch standardisation committee (NEN) on acoustics in Buildings

2000-2008 : member of the board of the Acoustical Society of the Netherlands, president of this society from 2004 to 2008.

2009 : co-chair of the NAG/DAGA2009 conference in Rotterdam

2009-now : convenor of the ISO working group on the revision of ISO 354 (absorption measurement in the reverberation room)

memberships:

Member of the NAG (Acoustical society of the Netherlands), DEGA (Deutsche Geselschaft für Akustik) and ASA (Acoustical Society of America) 


\section{Appendix 3 \\ List of publications}

Related to the subject

Reviewed publications:

- M. Vercammen, Sound Reflections from Concave Spherical Surfaces. Part I: Wave Field Approximation, Acta Acustica 96(2010)

- M. Vercammen, Sound Reflections from Concave Spherical Surfaces. Part II: Geometrical Acoustics and Engineering Approach, Acta Acustica 96(2010)

Other publications:

- M. Vercammen, Reflections of sound from concave surfaces, proceedings symposium on room acoustics, Seville, Sept. 2007

- M. Vercammen, Durch gekrümmte Flächen modifizierte Schallfelder, Daga Dresden 2008

- M. Vercammen, The reflected sound field by curved surfaces, Proc. Acoustics08, Paris, 2008

- M. Vercammen, Reflection of sound by concave surfaces, ICA, Sydney, 2010

\section{Other topics}

Reviewed publications:

- M.L.S. Vercammen, H.J. Martin, W.M.J. Cornelissen, Application of the intensity Measurement Technique to Building Acoustics and the influence of an Absorbing Partition wall on the Measured Intensity, Applied Acoustics, 23 (1988) 45-62.

- M.L.S. Vercammen, P.H. Heringa, Characterising Structure-borne Sound from Domestic Appliances, Applied Acoustics 28 (1989) 105-117.

Other publications (only as first author):

- M. Vercammen, B.P. Leaderer, R.N. Dietz, Influence of air flow on the performance of the perfluorcarbon tracer technique for measuring ventilation rates, proc. conf. Indoor Air, Berlijn, 1987.

- M.L.S. Vercammen, W.M. Schuller, Reduction of Low Frequency Vibration to Very low Levels, A test setup for a Measuring Device, Journal of Low Frequency Noise \& Vibration, vol. 6, nr. 2, 1987.

- M.L.S. Vercammen, P.H. Heringa, Contactgeluidafgifte van (huishoudelijke) toestellen, NAG journaal 95, 1988.

- M.L.S. Vercammen, P.H. Heringa, Laagfrequent geluid in onze woonomgeving, Geluid en omgeving, 1988, nr. 1.

- M.L.S. Vercammen, Setting Limits for low Frequency Noise, Journal of Low Frequency Noise \& Vibration, vol. 8, nr. 4, 1989.

- $\quad$ M.L.S. Vercammen, Grenzen aan laagfrequent geluid, Materialen, 1989, nr. 3.

- M.L.S. Vercammen, Hand- en armtrillingen door stotend handgereedschap, Polytechnisch tijdschrift, december 1989.

- M.L.S. Vercammen, Hand/arm trillingen van stotend handgereedschap, Ministerie SZW, publ. S 58-2, 1990.

- M.L.S. Vercammen, F.D. van der Ploeg, Het gebruik van trillingsmetingen voor de bepaling van het afgestraalde geluidvermogen, Geluid en Omgeving, juni 1990.

- M.L.S. Vercammen, P.H. Heringa, Low frequency noise transmission into Dwellings, Internoise 1990.

- M.L.S. Vercammen, P.H. Heringa, Berekening afstraalgraad verschillende constructies, IL-HR-13-04, 1991.

- M.L.S. Vercammen, Low-Frequency Noise Limits, Journal of Low Frequency Noise \& Vibration, vol. 11, nr. 1, 1992. 
- M.L.S. Vercammen, Toepassing van Maximum Length Sequences voor het meten van hoge geluidisolaties, NAG journaal 112, 1992.

- M.L.S. Vercammen, Soundproofing of sanitary systems, proc. Healthy buildings in relation to building services, 1992.

- M.L.S. Vercammen, The reciprocal substitution method for measuring the transmission of structure borne sound, Euronoise, 1992.

- M. Vercammen, Laagfrequent geluid, HMB lawaaibeheersing, 1992.

- M.L.S. Vercammen, Vibration in buildings, Internoise 93, Leuven.

- M.L.S. Vercammen, Th.W. Scheers, Sound Transmission through Suspended Ceilings, Internoise 93, Leuven.

- M.L.S. Vercammen, J.F.W. Koopmans, M.P.M. Luykx, Geluid en trillingen in de betonproduktenindustrie, Ministerie SZW, publ. S 57-11, 1993.

- $\quad$ M. Vercammen, Th.W. Scheers, Geluidisolatie in de utiliteitsbouw, NAG journaal 122, 1994.

- M. Vercammen, Geluidwering bij sanitaire installaties, klimaatbeheersing, 1994.

- M. Vercammen, De effectiviteit van diffusoren bij de bepaling van geluidabsorptie in de nagalmkamer, NAG Journaal, 1997

- M. Vercammen, Water hammer in domestic water supply installations, NAG Journaal, 2000 (in Dutch)

- M.L.S. Vercammen, The design of sound insulation measures for the dwellings around Amsterdam Airport Schiphol, NAG Journaal, 2002 (in Dutch)

- M.L.S. Vercammen, Contactgeluid van een Netkous; geluidafstraling van randstadrail, Congres Geluid en trillingen, 2003

- M.L.S. Vercammen, The design of sound insulation measures for dwellings around Amsterdam Airport Schiphol, internoise Prague, 2004

- M.L.S. Vercammen, Structure borne sound from a lightrail in a fishnet stocking, Internoise Rio de Janeiro, 2005

- M.L.S. Vercammen, R.van Schie, W.Beentjes, Akoestische kwaliteit van de woningbouw, NAG Journaal nr 175, 2005

- M. Vercammen, K.-H. Lorenz-Kierakiewitz, Vom Klopfgeist zu einer hervorragenden Akustik (1) - Die neue Tonhalle Düsseldorf: Die akustische Quadratur des Kreises, DAGA Braunschweig 2006

- M.Vercammen, T.Scheers, Absorption of open ceilings, Proc. ICA Madrid, 2007

- M.Vercammen, criteria for Low frequency noise, Proc. ICA Madrid, 2007

- M.Vercammen, G.Lasche, De Vercammen-curve: Wat is dat ?, congres Geluid en Trillingen 2007

- M.Vercammen, M.Lautenbach, The renovation of De Doelen Concert Hall, proceedings NAG/Daga 2009, Rotterdam

- M. Vercammen, How to improve the accuracy of the absorption measurement in the reverberation chamber?, proceedings NAG/Daga 2009, Rotterdam

- M. Vercammen, H.P.Tennhardt, Die akustische Geschichte der Staatsoper Unter den Linden, Daga 2010, Berlin

- M. Vercammen, Improving the accuracy of sound absorption measurement according to ISO 354, ISRA Melbourne, 2010 


\section{Dankwoord}

De aanleiding voor dit onderzoek was ruim zes jaar geleden een moeilijk, aanvankelijk schijnbaar onoplosbaar akoestisch probleem in de concertzaal Tonhalle te Düsseldorf. De uiteindelijk gekozen oplossing bleek succesvol. De klopgeest werd verdreven. Maar de reden waarom de aanvankelijk gekozen oplossing onvoldoende bleek was onduidelijk. Dat was het begin van een zes jaar durende speurtocht naar deze leemte in de zaalakoestische kennis. Het is een bijzonder voorrecht een dergelijk promotieonderzoek te kunnen doen, vooral vanwege de mogelijkheden die het biedt inhoudelijke discussies te kunnen voeren met onder andere de leden van de promotiecommissie. Het is niet het reisdoel maar vooral de reis waarom het gaat. Gedurende deze reis is bij mij de kennis en inzicht in het vakgebied akoestiek sterk toegenomen.

De afronding van dit onderzoek markeert ook 25 jaar werken bij Peutz. Een periode met vele uitdagingen, interessante onderzoeks- en adviesprojecten en boeiende contacten. Zowel tijdens die 25 jaren als gedurende dit promotieonderzoek hebben velen mij ondersteund, zowel praktisch als moreel.

Allereerst wil ik mijn familie, kinderen en vrienden, die veel aandacht tekort zijn gekomen, bedanken voor hun begrip. Marjolein dank ik voor de stimulans dit onderzoek te starten. Ten aanzien van mijn collega's bij Peutz wil ik mijn waardering uitspreken voor de samenwerking in die 25 jaar, een samenwerking die essentieel is om je als persoon op je plek te voelen, maar ook om gezamenlijk hoogwaardig werk af te kunnen leveren.

Ik wil graag Eddy Gerretsen en Heiko Martin bedanken voor hun support in het hele proces. Michael Vorländer heeft vrijwel het gehele traject als promotor gefungeerd, zijn enthousiasme heeft mij gestimuleerd dit werk te beginnen en af te ronden. Diemer de Vries ben ik dank verschuldigd voor de technische discussies en zinvolle suggesties en Bert Roozen, Armin Kohlraus en Gerrit Vermeir voor hun vele opbouwende opmerkingen op de concept versies van dit proefschrift.

Enkele dagen voor het drukken van dit proefschrift bereikte mij het tragische bericht van het overlijden van Renz van Luxemburg. Ik ben hem erkentelijk voor zijn steun voor deze promotie en de samenwerking binnen de NEN-commissie geluidwering in gebouwen. Ik wens zijn nabestaanden veel sterkte met het verwerken van dit verlies.

Hans van Koolwijk wil ik bedanken voor het mogen meten van zijn kunstwerk. De Rabobank en het bedrijf Nauerna ben ik eveneens erkentelijk voor het mogen uitvoeren van metingen.

Een aantal auteurs heeft, ofschoon de meeste onwetend, een bedrage geleverd aan het overzicht van praktijkgevallen in de literatuur in hoofdstuk 14.1, waarvoor ik hen evengoed bedank. 
Ich möchte mich herzlich beim ehemaligen Kulturdezernenten der Landeshauptstadt Düsseldorf, Herrn Hans-Heinrich Grosse-Brockhoff, dem damaligen Vorsitzenden der Gesellschaft der Freunde und Förderer der Düsseldorfer Tonhalle e.V., Herrn Dr. Edgar Jannott, und der damaligen Intendantin der Tonhalle, Frau Vera von Hazebrouck für das Vertrauen in unsere Arbeit und unsere Herangehensweise bei der raumakustischen Planung der Sanierung der Tonhalle bedanken. Der gleiche Dank gilt dem Architekten, Herrn Volker Weuthen für die gute und konstruktive Zusammenarbeit und die vorzügliche Umsetzung der raumakustischen Vorgaben.

Rob Metkemeijer wil ik hartelijk bedanken voor de steun bij het inwerken in zijn vakgebied, de zaalakoestiek, en in het kader van dit promotieonderzoek zeker ook voor het maken van het model waarmee de experimenten beschreven in hoofdstuk 11 zijn uitgevoerd (hij vindt nog dagelijks groene schilfertjes) en de support voor wat betreft het schaalmodelonderzoek van de Royal Albert Hall.

Hans van Heeswijk ben ik dank verschuldigd voor de metingen en analyses en Klaus-Hendrik Lorenz voor de metingen en samenwerking bij het Tonhalle projekt.

Maar vooral wil ik Margriet Lautenbach bedanken voor haar vele inhoudelijke suggesties, discussies, literatuurverwijzingen en kritische opmerkingen op de tekst. Zonder al deze personen zou het voltooien van dit werk niet mogelijk zijn geweest. 

